\author{
Universidade de São Paulo \\ Faculdade de Saúde Pública \\ Departamento de Epidemiologia
}

\title{
Acessibilidade de pessoas com deficiência a serviços de saúde em áreas do Estado de São Paulo - Projeto AceSS.
}

Shamyr Sulyvan de Castro

Tese apresentada ao Programa de Pós-Graduação em Saúde Pública para obtenção do título de doutor em Saúde Pública.

Área de concentração: Epidemiologia

Orientador: Prof. Dr. Chester Luiz Galvão Cesar

São Paulo

2010 


\section{Acessibilidade de pessoas com deficiência a serviços de saúde em áreas do Estado de São Paulo - Projeto AceSS.}

\section{Shamyr Sulyvan de Castro}

Tese apresentada ao Programa de Pós-Graduação em Saúde Pública da Faculdade de Saúde Pública da Universidade de São Paulo para obtenção do título de Doutor em Saúde Pública.

Área de concentração: Epidemiologia

Orientador: Prof. Dr. Chester Luiz Galvão Cesar

\section{São Paulo}

2010 
É expressamente proibida a comercialização deste documento tanto na sua forma impressa como eletrônica. Sua reprodução total ou parcial é permitida exclusivamente para fins acadêmicos e científicos, desde que na reprodução figure a identificação do autor, título, instituição e ano da tese. 


\section{Agradecimentos}

Os meus sinceros agradecimentos ao Prof. Dr. CHESTER LG CeSAR pela orientação e estímulo que sempre direcionou à realização deste trabalho e à minha carreira profissional; à Profa. Dra. Alarcos CIEZA, pela atenção e respeito que dedicou a mim durante o período em que trabalhamos juntos; aos professores que compõem a banca de avaliação desta pesquisa, pelas sugestões e comentários na qualificação e defesa deste doutorado.

À CAMILA, pela dedicação, paciência e compreensão que sempre mostrou em relação à minhas ausências e mudanças de endereço; espero que continuemos trilhando o mesmo caminho, para que eu possa ter sempre sua presença e seu apoio.

À minha família, que sempre está presente, mesmo distante, e com quem divido com muito prazer esta conquista.

Aos amigos NEUBER e RoDRIGO, sempre próximos e trazendo alegria e descontração para os dias de trabalho mais pesado.

Aos outros amigos, ausentes ou presente, próximos ou distantes, que acompanharam todo processo de realização desta pesquisa e deram seu apoio.

Aos funcionários e professores da FSP que sempre me atenderam com respeito e educação.

Aos entrevistadores/pesquisadores que colaboraram na execução deste estudo e às pessoas que concederam um pouco de seu tempo para responder as entrevistas realizadas.

Agradeço especialmente ao Conselho Nacional de Desenvolvimento Científico e Tecnológico - CNPq (proc. 409724/2006-8) pelo financiamento da fase de campo desta pesquisa; à Fundação de Amparo à Pesquisa do Estado de São Paulo - FAPESP (proc. 2006/59655-2) pela bolsa de doutorado a mim concedida e ao Serviço Alemão de Intercâmbio Acadêmico (DAAD) que juntamente com o CNPq financiou meu estágio de doutorado-sanduíche na Alemanha (proc. 290006/2008-2). 


\section{Epígrafe}

"Sólo le pido a Dios

que el dolor no me sea indiferente,

que la reseca muerte no me encuentre

vacío y solo, sin haber hecho lo suficiente.

Sólo le pido a Dios

que lo injusto no me sea indiferente,

que no me abofeteen la otra mejilla,

después que una garra me arañó esta suerte."

(Sólo le pido a Dios - León Gieco)

"Somos nós que fazemos a vida

Como der ou puder ou quiser

Sempre desejada, por mais que esteja errada

Ninguém quer a morte, só saúde e sorte..."

(O que é, o que é - Gonzaguinha)

"Todo mundo ama um dia todo mundo chora,

Um dia a gente chega, no outro vai embora

Cada um de nós compõe a sua história

Cada ser em si carrega o dom de ser capaz

E ser feliz."

(Tocando em frente - Almir Sater e Renato Teixeita) 
Castro SS. Acessibilidade de pessoas com deficiência a serviços de saúde em áreas do Estado de São Paulo - Projeto AceSS [tese de doutorado]. São Paulo: Faculdade de Saúde Pública da Universidade de São Paulo; 2010.

\section{Resumo}

Introdução. As pessoas com deficiência são um grupo populacional que em virtude de suas condições de saúde, apresentam algumas dificuldades na acessibilidade aos serviços de saúde. Objetivo. Estudar os problemas de acessibilidade de pessoas com deficiências físicas, visuais e/ou auditivas aos serviços de saúde em áreas do Estado de São Paulo, bem como as variações existentes segundo o tipo de deficiência e variáveis sociodemográficas. Material e Métodos. Estudo transversal com entrevistas domiciliares realizado em duas fases distintas. Fase I: entrevistas com 25 indivíduos com alguma deficiência, por meio de questionário qualitativo, com perguntas abertas, sobre os problemas de acessibilidade enfrentados no uso dos serviços de saúde, analisadas pelo método do "Discurso do Sujeito Coletivo". Fase II: entrevistas com 333 pessoas com deficiência, por meio de questionário fechado, que teve como tema os problemas de acessibilidade aos serviços de saúde, além de outros. Esse questionário originou-se dos resultados da Fase I, de perguntas encontradas em outras pesquisas e de conceitos da Classificação Internacional de Funcionalidade, Incapacidade e Saúde (CIF). Os dados foram analisados com frequências simples, Qui-quadrado, Qui-Quadrado de tendência e regressão de Poisson com variância robusta. O software utilizado na análise é o Stata 9.2®. Resultados. Os principais problemas de acessibilidade citados pelos entrevistados são o tempo de espera para ser atendido, a ausência de rampas, de estacionamento e de sanitários adaptados, de salas de espera com lugares insuficientes, falta de cadeira de rodas para pacientes e salas bloqueadas por obstáculos. Problemas de acessibilidade aos serviços de saúde foram relatados por $15,92 \%$ dos 333 entrevistados. As pessoas com paralisia, amputação ou deficiências múltiplas, as pessoas com idade menor que 78 anos de idade ou as que precisavam de ajuda para ir ao serviço de saúde, tiveram mais problemas que os outros. Dos 160 entrevistados 
com alguma deficiência auditiva, 35\% relataram problemas para ouvir e entender o que foi dito pelos profissionais de saúde. Conclusões. Problemas como tempo de espera, ausência de rampas e/ou estacionamento, dificuldades de comunicação com os profissionais de saúde e acessibilidade às salas do serviço de saúde foram relatados pelos entrevistados. O estudo dos problemas de acessibilidade de pessoas com deficiência aos serviços de saúde pode ajudar na orientação e na criação de políticas e de programas específicos para esse grupo populacional, visando a melhoria da sua saúde geral e qualidade de vida, por meio do incremento da acessibilidade aos serviços de saúde.

Descritores: Perfil de Saúde; Pessoas com Deficiência; Inquéritos de Saúde; Acessibilidade aos Serviços de Saúde. 
Castro SS. Accessibility by people with disabilities to health services in areas of São Paulo state - AceSS Project. [doctoral thesis]. São Paulo (BR): Faculty of Public Health. São Paulo University; 2010.

\section{Abstract}

Introduction. People with disabilities are a population group that have some difficulties in accessibility to health services due to their health conditions. Objective. To study the problems of accessibility of people with physical, hearing and/or visual disabilities to health services in areas of São Paulo state, as well as some discrepancy according to the type of disability and socio-demographic variables. Methods. Cross-sectional study with household interviews conducted in two phases. Phase I: 25 persons with disability were interviewed with an open questionnaire. The interviews were taped, transcribed and the method of data analysis was the Discourse of the Collective Subject. The program Qualiquantsoft was used to analyze the qualitative data. Phase II: 333 persons with disabilities were interviewed with a closed questionnaire that was derived from the original results of Phase I, questions found in other questionnaires and concepts of the International Classification of Functioning, Disability and Health (ICF). Data were analyzed using simple frequencies, $\chi 2$-test, $\chi 2$-test for trend and Poisson regression with robust variance. The software used in the analysis was Stata 9.2®. Results. The main accessibility problems cited by respondents was the waiting time to be served; no ramps, parking and/or adapted toilets; waiting room with insufficient places; lack of wheelchairs for patients; and rooms blocked by obstacles. Problems of accessibility to health services were reported by $15.92 \%$ of the 333 respondents. People with paralysis or amputation or multiple disabilities, as well as those younger than 78 years of age or those who needed help to go to health service, had more problems than others. $35 \%$ of the 160 interviewed reported hearing problems to listen and understand what had been said by health professionals. Conclusions. Problems such the waiting time; no ramps; inappropriate parking; poor communication with health professionals and accessibility to the rooms of the health service were reported. The 
determination of these issues may influence the creation of specific policies and programs for this population group, aiming to improve their overall health and quality of life, through the increment of the accessibility to health services.

Key Words: Health profile; Persons with Disabilities; Surveys; Health services accessibility 


\section{Índice}

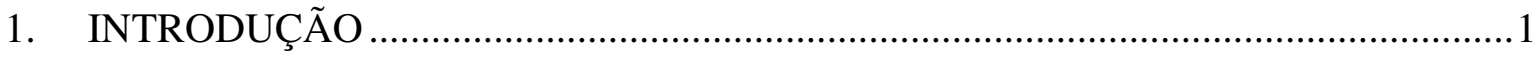

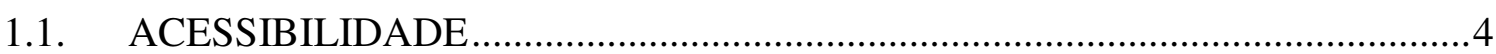

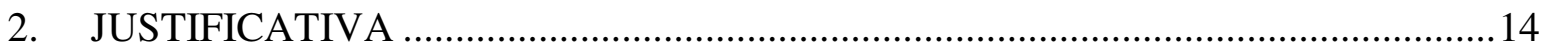

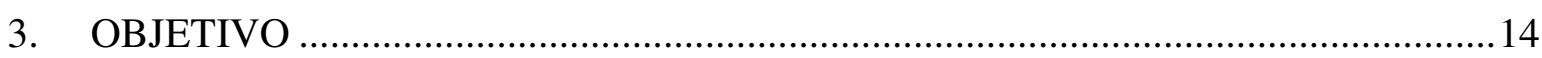

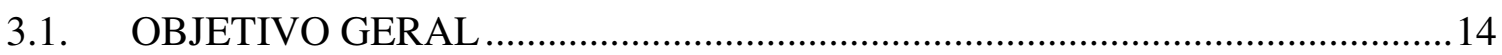

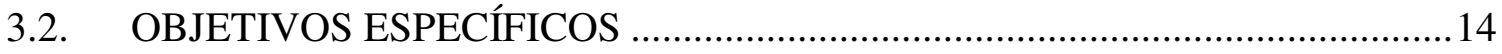

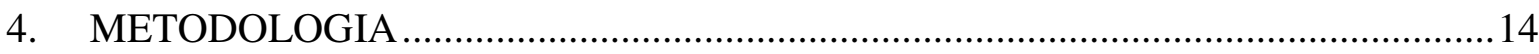

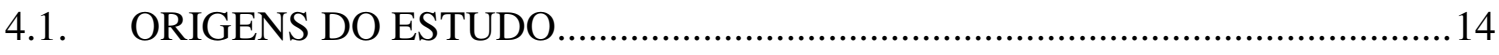

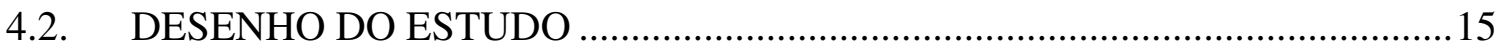

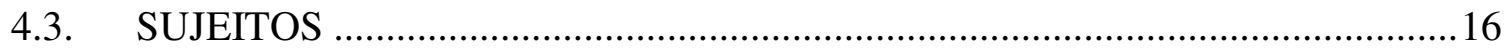

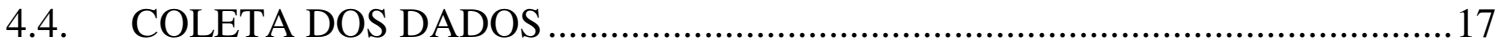

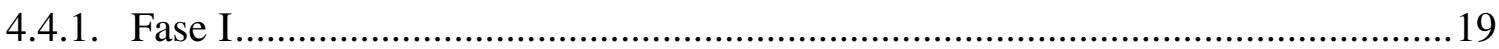

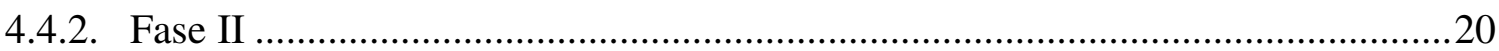

4.5. ANÁLISE DOS DADOS

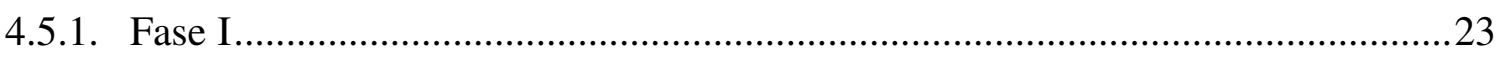

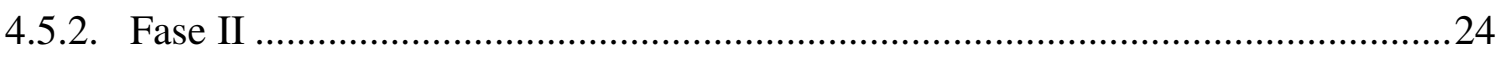

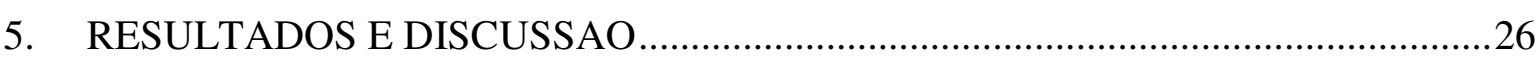

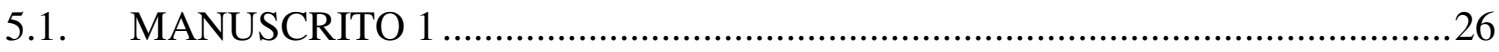

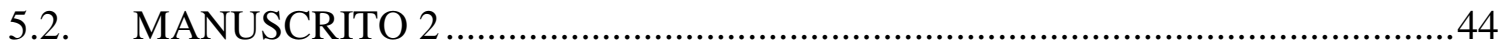

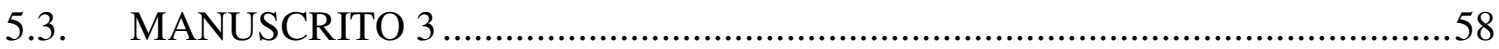

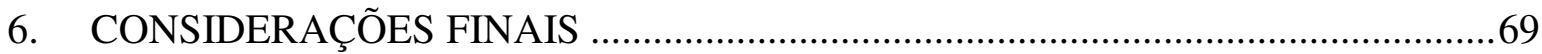

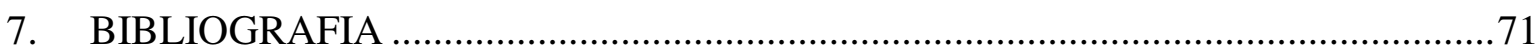

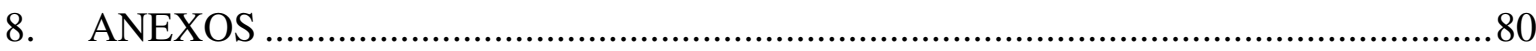

8.1. ANEXO I - QUESTIONÁRIO ABERTO UTILIZADO NA FASE I DO

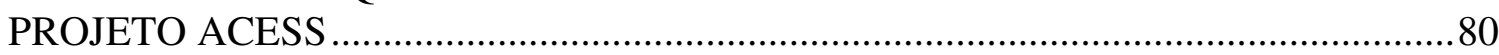

8.2. ANEXO II - QUESTIONÁRIO FECHADO UTILIZADO NA FASE II

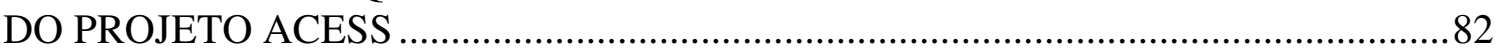

8.3. ANEXO III - MATERIAL DE DIVULGAÇÃO DO CONSELHO MUNICIPAL DA PESSOA COM DEFICIÊNCIA ….................................................. 127

8.4. ANEXO IV - GUIA DE ENCAMINHAMENTOS PARA PESSOAS COM DEFICIÊNCIA E MOBILIDADE REDUZIDA. 


\section{Lista de Figuras, Tabelas e Quadros}

Figura 1 - Esquema representativo dos componentes principais da acessibilidade..... 6

Figura 2 - Descrição esquemática do Paradoxo das Barreiras..................................... 7

Figura 3 - Descrição do processo de obtenção da amostra final e do total de entrevistas, Projeto AceSS, São Paulo, 2007

Tabela 1 - Distribuição dos indivíduos entrevistados na Fase I segundo deficiência, sexo e média de idade, Projeto AceSS, São Paulo, 2007

Tabela 2 - Características sociodemográficas da amostra de sujeitos estudados, Projeto AceSS, São Paulo, 2007.

Quadro 1 - "Modelos" de compreensão da experiência da deficiência. 2

Quadro 2 - Conteúdo temático dos blocos dos questionários, segundo pesquisa, São Paulo, 2007.

Quadro 3 - Composição do questionário fechado do estudo AceSS, suas variáveis, temas, número de questões e especificidades segundo tipo de deficiência, 2008. .... 22 


\section{INTRODUÇÃO}

As pessoas com deficiência têm tido diferentes representações sociais ao longo da história da humanidade, desde a Grécia antiga até os tempos de hoje (ROSE, 2006), assumindo, na atualidade, importância até então não alcançada. Os profissionais de saúde, por sua vez, começaram a voltar sua atenção às pessoas com algum tipo de deficiência a partir do século XVIII, com progressivo interesse até a evolução teórica e prática alcançada pela Classificação Internacional de Doenças e pelo surgimento da Classificação Internacional de Incapacidade e Funcionalidade (CIF) (AMIRALIAN et al., 2000).

Nesse contexto, as pessoas com deficiência vêm se configurando como um grupo populacional mais consciente e participativo, com marcada militância em favor de sua causa. No Brasil, essa militância ocorreu primariamente pelas ações de pais e parentes, nos anos 1960, contra a segregação e em favor da convivência social. Essas ações resultaram em reportagens sobre os protestos na imprensa brasileira no final da década de 70, época em que a mídia internacional também veiculava material relacionado às pessoas com deficiência. A mobilização global em favor das pessoas com deficiência favoreceu a proclamação da Declaração dos Direitos das Pessoas Deficientes, entre outros documentos e ações positivas em torno do tema (FRANÇA e PAGLIUCA, 2009). Atualmente, a questão das pessoas com deficiência na sociedade e sua inclusão social têm recebido atenção especial em diversos países.

Por outro lado, a definição do termo "deficiência" tem sido objeto de inúmeras discussões entre os diversos pesquisadores da área, sendo uma construção teórica de pelo menos dois séculos (ROCHA, 2006). GRÖNVIK (2007) relata que esse assunto é controverso e que diferentes abordagens podem ser feitas considerando fatores humanistas, culturais e terminológicos. Por haver mais de um "modelo" que trabalhe o tema, as incongruências são frequentes. No Quadro 1, examinamos alguns modelos de compreensão da deficiência. 
Quadro 1 - "Modelos" de compreensão da experiência da deficiência.

\begin{tabular}{|c|c|c|}
\hline Modelo & Descrição & Referência \\
\hline Médico & $\begin{array}{l}\text { A deficiência é causada por uma doença ou } \\
\text { trauma, sendo sua solução relacionada aos } \\
\text { profissionais da área. A deficiência é um } \\
\text { "desvio" da normalidade. Neste modelo a } \\
\text { deficiência é considerada somente no âmbito } \\
\text { individual. }\end{array}$ & SEULLION, 1999 \\
\hline Reabilitação & $\begin{array}{l}\text { Baseado no modelo médico e assim como ele, } \\
\text { centra seus argumentos no indivíduo e os } \\
\text { responsabiliza pela superação de sua } \\
\text { deficiência. }\end{array}$ & $\begin{array}{l}\text { LOLLAR } \\
\text { CREWS, } 2003 .\end{array}$ \\
\hline Social & $\begin{array}{l}\text { Associa a deficiência às questões sociais. } \\
\text { Atribui esse acontecimento à falta de } \\
\text { consciência e interesse da sociedade. Este } \\
\text { modelo não prevê que fatores como doenças e } \\
\text { traumas integrem a concepção de deficiência, } \\
\text { considerando a sociedade como sua causa. }\end{array}$ & $\begin{array}{l}\text { SHAKESPEARE e } \\
\text { WALSON, } 1997 .\end{array}$ \\
\hline Interface & $\begin{array}{l}\text { Baseia-se na premissa de que a deficiência } \\
\text { existe a partir da interface entre os fatores } \\
\text { biológicos (doenças) e o ambiente. A } \\
\text { deficiência é vista como uma experiência de } \\
\text { vida em que a pessoa com deficiência tem o } \\
\text { controle de suas decisões. }\end{array}$ & GOODALL, 1995 \\
\hline $\begin{array}{l}\text { Bio-psico- } \\
\text { social }\end{array}$ & $\begin{array}{l}\text { A deficiência é vista como produto de uma } \\
\text { combinação entre fatores de níveis físicos, } \\
\text { emocionais e ambientais. }\end{array}$ & $\begin{array}{l}\text { LUTZ e BOWERS., } \\
2005\end{array}$ \\
\hline
\end{tabular}

Baseado em SMELTZER, 2007

Conforme o Quadro 1, a compreensão de deficiência parte de uma abordagem inicialmente voltada para a doença, com seu foco evoluindo da reabilitação e suas causas sociais, em direção a uma interação entre essas causas e outras mais ligadas ao campo biológico, chegando-se ao modelo bio-psico-social, que agrega os anteriores, considerando a interação entre esses fatores. A tendência em se aceitar a deficiência como um fato bio-psico-social, segue os conceitos publicados e preconizados pela CIF, Classificação Internacional de Funcionalidade, Incapacidade e Saúde, lançada em 2001 pela Organização Mundial de Saúde (OMS, 2003). A CIF considera que as deficiências são problemas na função ou estrutura corporal, tais como um desvio ou uma perda significativos, enquanto a incapacidade seria um 
termo genérico para deficiências, limitações de atividades e restrições à participação (Di NUBILA e BUCHALLA, 2008), com foco mais direcionado à interação com o meio ambiente.

A vida de uma pessoa com deficiência altera-se consideravelmente após a ocorrência de um evento que leve a um processo incapacitante. As atividades de envolvimento social são prejudicadas e a restrição na participação social pode conduzir a pessoa à reclusão domiciliar. Essa reclusão reforça a diminuição dos estímulos, pela falta de experiências extradomiciliares, o que, por consequência, pode aumentar a dependência de outras pessoas e prejudicar a auto-estima (FÄNGE et al., 2002). Outras atividades, antes comuns, como vestir-se ou alimentar-se, passam a representar grandes obstáculos. Cuidados especiais devem ser direcionados aos aspectos nutricionais, à mobilidade, à alimentação, ao trânsito intestinal, à incontinência, à comunicação com outras pessoas, à higiene oral, à vida social e ao trabalho, entre outros (BEANGE, 1996; CHAVES et al., 2004; DETELS et al., 2004).

Diversos pesquisadores ressaltam que os indivíduos com deficiências diversas (físicas ou intelectuais) estão mais expostos a comorbidades associadas à sua deficiência (SEEKINS et al., 1994; BEANGE, 1996; RAVESLOOT et al., 1997; COYLE et al., 2000; CHRISTENSEN et al., 2005; RAVESLOOT et al., 2005; HARRISON, 2006; NOSEK et al., 2006). DEJONG (1997) relata que, entre os motivos que ocasionam maior prevalência de comorbidades entre as pessoas com deficiência, cita-se o que o autor chama de "thinner margin of health", que se pode entender por um limiar de saúde mais tênue, ou seja, uma situação de saúde mais instável que a dos indivíduos sem deficiências. $\mathrm{O}$ autor ressalta que as pessoas com deficiência não são mais doentes que os outros e sim que a sua deficiência as torna mais vulneráveis às doenças.

Um segundo motivo que contribuiria para as comorbidades entre as pessoas com deficiência é que elas não têm as mesmas oportunidades de acesso aos serviços de manutenção e prevenção da saúde que os outros (BEANGE, 1996; DEJONG, 1997).

Outro fator apontado por DEJONG (1997) como predisponente às comorbidades entre as pessoas com deficiência é a precocidade com que as doenças 
crônicas ocorrem. Esse fato tem sua origem na diminuição da mobilidade das pessoas com deficiências físicas e visuais, principalmente. A menor mobilidade tornaria mais fácil o aparecimento de doenças como diabetes e hipertensão.

As perdas funcionais secundárias são outro fator importante que contribui para o aparecimento de doenças, gerando maior exposição a fatores patógenos, causadores de morbidades. Assim, uma pessoa com tetraplegia e acamada pode ter seu funcionamento pulmonar alterado, facilitando o aparecimento de pneumonias, devido à imobilização no leito. Por último, a necessidade de cuidados de saúde constantes e de equipamentos (próteses, órteses, ventiladores mecânicos, etc.) pode proporcionar o aparecimento de outras doenças. Uma pessoa com deficiência que necessite usar sonda uretral, por exemplo, é mais sujeita a infecções urinárias (DEJONG, 1997).

As pessoas com deficiência também necessitam de transporte adequado e adaptações para se locomover de um ponto a outro sozinhas, já que nem sempre podem contar com ajuda para isso (LAWTHERS et al., 2003). Entretanto, em um artigo norte-americano, há relatos de que as equipes que fazem o transporte dos indivíduos com deficiências nem sempre têm o devido preparo e tampouco dispõem de equipamentos adequados (SUTTON e DEJONG, 1998).

Esse grupo populacional, como qualquer outro, deve realizar periodicamente exames preventivos para acompanhamento do seu estado geral de saúde e detecção precoce de possíveis agravos à saúde (LIANZA, 1995; SUTTON e DEJONG, 1998). Entretanto, o ambiente pode representar um obstáculo àqueles com algum tipo de deficiência, levando à não utilização dos serviços de saúde necessários. Conforme KROLL et al. (2006), a subutilização dos serviços de saúde resulta em prejuízos para as pessoas, principalmente se elas têm algum tipo de deficiências.

\subsection{ACESSIBILIDADE}

O conceito de acessibilidade é bastante discutido e tem diversas abordagens, como as de Donabedian, discutido por CUNHA e VIERA-da-SILVA, (2010); de Maxwell (MERREL et al., 2006); de Harding, de Aday e Andersen e de Joseph e Philips (WILSON e ROSEMBERG, 2004); e, também, a do modelo de interação 
entre meio ambiente e pessoa (WERNGREM-ELGSTRÖM et al., 2009), entre outras. Para a presente pesquisa, consideramos o conceito de FRENK (1985), por se adequar melhor à realidade do Brasil e de seu sistema de saúde.

O termo "acessibilidade" aos serviços de saúde é frequentemente confundido e substituído por outros usados na área, como o "acesso" e a "disponibilidade", conforme lembra FRENK (1985). Este autor desfaz as confusões a respeito desses conceitos delimitando e definindo acesso como "a capacidade de um cliente ou grupo de clientes para buscar e obter atenção em saúde"; e disponibilidade como "presença dos recursos de atenção em saúde tendo em conta sua produtividade, ou seja, sua capacidade para produzir serviços” (FRENK, 1985). Após explanar sobre os dois tópicos anteriores o autor define acessibilidade como "o grau de ajuste entre as características dos recursos de atenção em saúde e as características das populações, no processo de busca e obtenção da atenção em

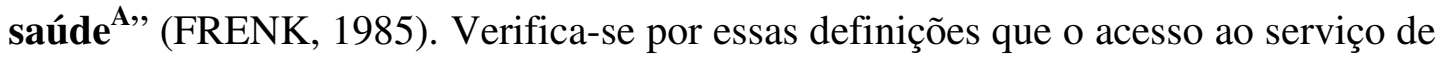
saúde está mais voltado às características da população e de disponibilidade de serviços de saúde do que às características dos recursos de atenção à saúde (FRENK; 1985). Assim, percebe-se que a acessibilidade compreende o universo ao redor e a interface entre a disponibilidade e o acesso aos serviços de saúde.

FRENK (1985) e RICKETTS e GOLDSMITH (2005) apresentam, ainda, outros conceitos importantes para o completo entendimento da acessibilidade. São eles: "resistência", que seria o conjunto de obstáculos enfrentados na busca e na obtenção dos serviços de saúde; e "disponibilidade efetiva", que pode ser entendida pela disponibilidade corrigida segundo a resistência à obtenção dos serviços de saúde.

Pelas definições anteriores, fica claro que a acessibilidade não pode ser entendida puramente como a relação entre disponibilidade e acesso, pois o autor introduz um novo e importante componente, a resistência. O esquema da Figura 1 explica melhor o que se descreveu anteriormente.

\footnotetext{
${ }^{\mathrm{A}}$ Traduções do autor
} 
Figura 1 - Esquema representativo dos componentes principais da acessibilidade.

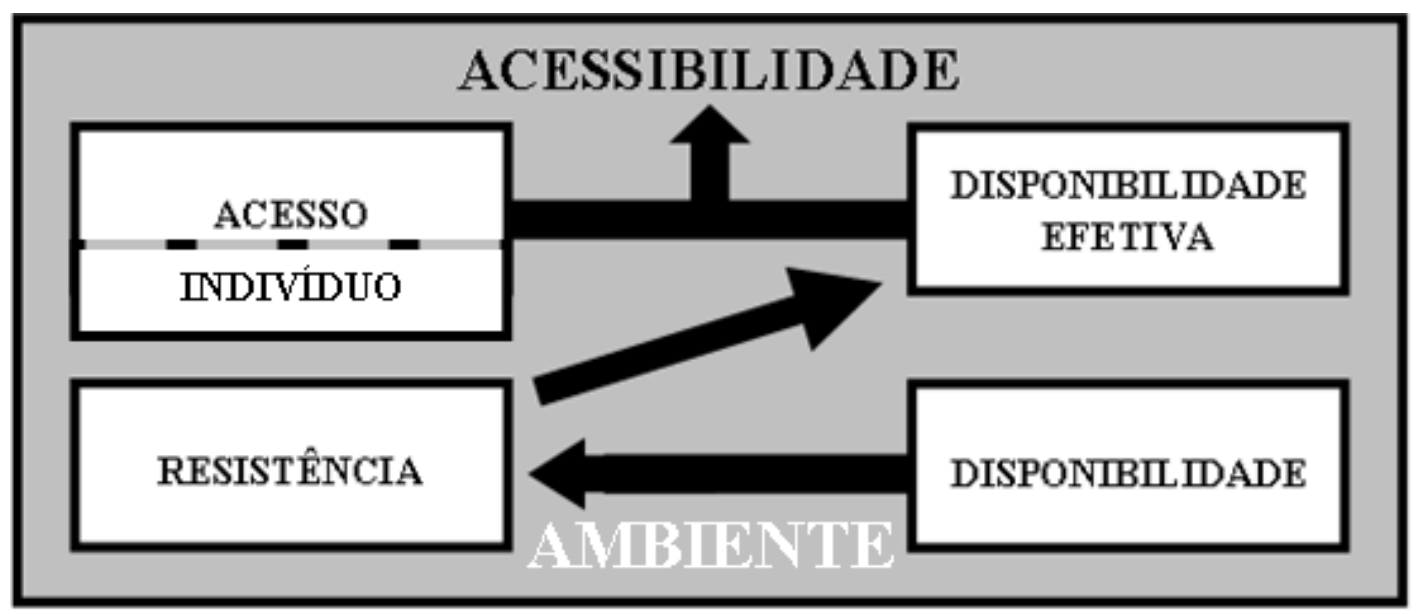

Baseado em FRENK (1985).

No esquema apresentado na Figura 1, percebe-se que os componentes (1) disponibilidade, (2) resistência e (3) disponibilidade efetiva têm relação direta com o meio ambiente. O acesso é uma característica mais ligada ao indivíduo, mas que é resultante de sua interação com o ambiente, significando, na verdade o uso final do serviço de saúde.

Os caminhos apontados por FRENK (1985) dentro da complexidade do termo acessibilidade sugerem uma interação dos conceitos apresentados. Pensando de maneira simplificada poderíamos representar o esquema anterior em uma fórmula matemática da seguinte maneira:

$[(\mathbf{A}-\mathbf{B})+\mathbf{C}]=\mathbf{D}$, onde

$\mathrm{A}=$ disponibilidade

$\mathrm{B}=$ resistência

$\mathrm{C}=$ acesso

$\mathrm{D}=$ acessibilidade

Sendo que (A - B) é igual à disponibilidade efetiva.

Assim, a acessibilidade seria o resultado da disponibilidade efetiva dos serviços de saúde somada ao acesso por parte da população. 
A resistência apresentada anteriormente tem especial importância quando se estuda a acessibilidade de pessoas com deficiência aos serviços de saúde. Ela pode ser entendida como a dificuldade encontrada na obtenção dos serviços de saúde. O que ocorre é que, em virtude do quadro de saúde proporcionado pela deficiência e das comorbidades advindas desse quadro, mais e mais dificuldades interpõe-se entre a pessoa com deficiência e o serviço de saúde, aumentando, assim, a resistência (WILSON e ROSENBERG, 2004). Esse aumento da resistência pelas barreiras ambientais, que gera uma diminuição da participação da pessoa foi nomeado por WHITENECK e DIJKERS (2009) “O Paradoxo das Barreiras”, que é um fenômeno em que o aumento das barreiras diminui a participação da pessoa com deficiência. Por outro lado, a diminuição dessas barreiras aumenta a participação, mostrando uma relação inversamente proporcional (Figura 2).

Figura 2 - Descrição esquemática do Paradoxo das Barreiras ${ }^{B}$.

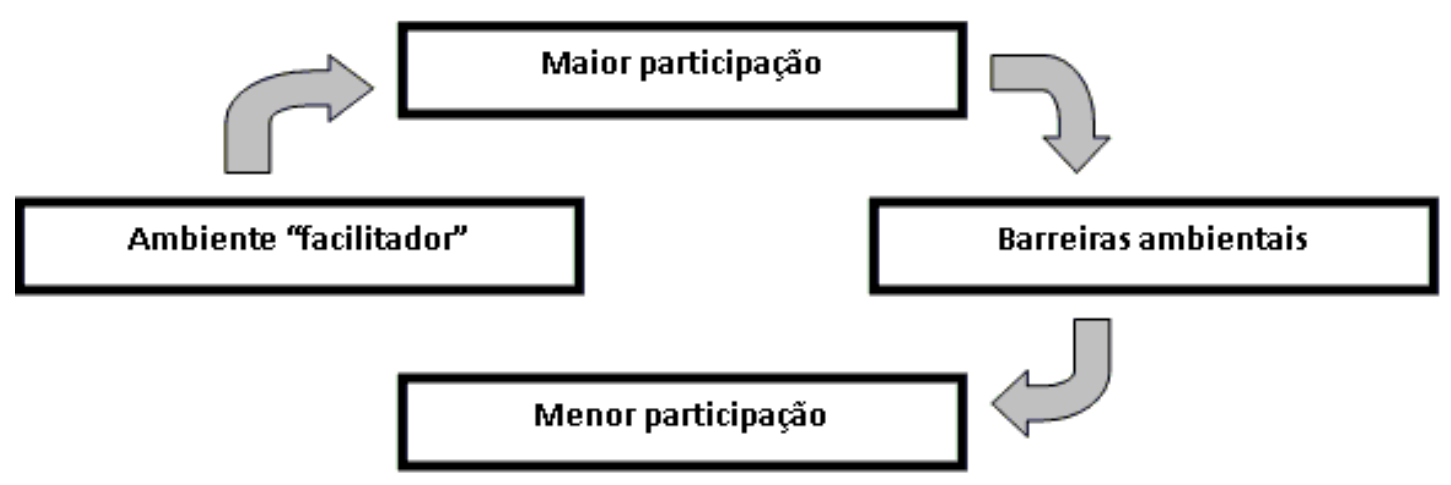

Por apresentarem características um pouco diferentes da população em geral, as pessoas com deficiência enfrentam mais barreiras a serem transpostas na sociedade, que podem ser geográficas, comportamentais, arquitetônicas e financeiras, entre outras. KROLL et al. (2006) definem as barreiras enfrentadas pelas pessoas com deficiência como os fatores que impedem que a pessoa faça uso do serviço de saúde em sua totalidade em níveis satisfatórios. LIPSON e ROGERS (2000) relatam que, nos EUA, as pessoas com deficiência são uma minoria exposta a restrições no

\footnotetext{
${ }^{\text {B }}$ Traduzido e adaptado de WHITENECK e DIJKERS; 2009.
} 
acesso à educação e ao bem-estar, sendo ainda estigmatizadas e marginalizadas por outras pessoas em sua comunidade. Essas seriam outras modalidades de barreiras impostas às pessoas com deficiência, com natureza diversa das barreiras físicas, sendo caracterizadas como barreiras sociais ou comportamentais.

Mesmo tendo um perfil de saúde que necessite maior atenção, as pessoas com deficiência enfrentam alguns problemas para obter os serviços de saúde necessários. LAWTHERS et al. (2003) e outros estudiosos citam quatro dificuldades principais:

- barreiras físicas e de transporte, que são também citadas por GRAY et al. (2003); TALBOT et al. (2004) e THAPAR et al. (2004).

- acesso limitado a equipamentos e ajudas técnicas ou equipamentos de ajuda (SCHOPP et al., 2002; LAWTHERS et al., 2003).

- limitação do acesso a medicações e especialistas (LAWTHERS et al., 2003).

- dificuldade de acesso a atendimento de cuidados pessoais (LAWTHERS et al., 2003).

PEAT (1997) relata que a comunicação e o livre acesso físico garantem às pessoas com deficiência a completa utilização dos serviços de saúde, da mesma maneira que às outras pessoas. As pessoas com deficiência auditiva, por exemplo, enfrentam dificuldades em consultas médicas e odontológicas ou outros serviços de saúde em que os profissionais usam máscaras. O uso desses equipamentos de proteção inviabiliza a leitura labial que esses indivíduos usam para se comunicar, impossibilitando o entendimento, por parte do paciente, do que o profissional de saúde tem a dizer (BEANGE, 1996; HARMER, 1999; UBIDO et al., 2002; STEINBERG et al., 2006). Essa dificuldade soma-se ao fato de ser complicada a transmissão de mensagens da pessoa com alguma deficiência relacionada à fala para o profissional de saúde (STEINBERG et al., 1998; BRANIGAN et al., 2001; IEZZONI et al., 2004), que, quase sempre não tem treinamento para o atendimento de pessoas com necessidades especiais de comunicação (BEANGE, 1996).

Em um estudo com crianças com deficiências físicas e intelectuais, WHARTON et al. (2005) colheram relatos dos pais, que apontaram algumas 
dificuldades encontradas por eles no acesso aos serviços de saúde do Reino Unido. Os principais problemas indicados foram:

- preparação para a ida ao hospital (preparação do paciente, veículo apropriado, etc.)

- horários de atendimento (a rigidez dos horários dificulta o acesso no caso de pacientes mais complicados ou que exijam uma maior mobilização de pessoal e equipamentos)

- estacionamento no local de atendimento (dificuldades com estacionamento próximo à entrada do estabelecimento de saúde, prejudicando o translado do paciente e sua entrada no local)

- espaço físico (pacientes que faziam uso de cadeiras de rodas encontravam dificuldades com a mobilidade interna nos ambientes dos serviços de saúde)

- sala de espera (falta de espaço para cadeira de rodas e excesso de barulho)

- dificuldade dos profissionais de saúde em entender e conhecer a deficiência (muitos médicos tinham dificuldade em determinar se um problema de saúde era ligado à deficiência ou não)

- internação (quartos com dimensões diminutas e inadequadas)

Além dos obstáculos físicos, as pessoas com deficiência ainda encontram outros obstáculos não-físicos no acesso aos serviços de saúde. SCHOPP et al. (2002) pesquisaram a acessibilidade aos serviços de saúde por mulheres com deficiência e relatam que existem basicamente três tipos de barreiras não-físicas para essas pessoas:

- Barreiras de informação: o indivíduo com deficiência é privado de informações a respeito dos serviços de saúde e sobre como acessá-los mais facilmente. Esse tipo de barreira é prejudicial, uma vez que priva as pessoas de informações que poderiam facilitar seu acesso ao serviço de saúde. Nesse sentido, uma pessoa com deficiência visual fica prejudicada quando o principal meio de divulgação de uma campanha de saúde é a comunicação visual (cartazes, figuras, etc.). 
- Barreiras de comportamento: a pessoa com deficiência pode deparar-se com comportamentos impróprios dos profissionais de saúde. O profissional de saúde de um hospital ou unidade básica de saúde nem sempre está adequadamente capacitado para receber uma pessoa com deficiência. Esse fato pode ocorrer por insegurança ou despreparo do funcionário, ou seja, o médico pode ter receio de atender uma pessoa com deficiência julgando que ela seja "diferente" das outras, quando, na verdade a doença que ele tem ou o motivo que o levou a procurar atendimento é igual ao de outras pessoas. O profissional de saúde pode não entender que o que leva uma pessoa com deficiência a procurar o serviço de saúde é uma doença, ou mesmo uma ação de prevenção, e não a deficiência.

- Barreiras financeiras: o custo do atendimento é alto para esses pacientes, o que pode gerar uma menor utilização dos serviços de saúde por motivos econômicos. As pessoas com deficiência podem enfrentar dificuldades ligadas ao pagamento de transporte, medicamentos, consultas particulares, entre outros, dificultando o uso adequado dos serviços de saúde necessários. Além disso, há ainda a dificuldade de pagamento de planos de saúde. No Brasil, existe uma legislação que beneficia as pessoas com deficiência com ajuda financeira, o que minimiza essa barreira, entretanto, ela não é completamente eliminada.

GRABOIS et al. (1999) em estudo feito com médicos do Estado americano do Texas, encontraram problemas no acesso físico aos consultórios médicos. $\mathrm{O}$ desenho arquitetônico dos locais de atendimento não atendia aos preceitos para livre acesso de pessoas com deficiência, impondo barreiras entre a pessoa e os serviços de saúde. Esse problema é tão importante que, em 1997, JONES e TAMARI (1997) publicaram um artigo estabelecendo medidas que os médicos deveriam adotar para tornar os consultórios totalmente acessíveis. CURRIE (1997) assegura que a facilidade na acessibilidade ao estabelecimento de saúde é de crucial importância para o bom uso do sistema de saúde. COPPEL et al. (1999) perceberam que adaptações arquitetônicas deveriam ser realizadas nos estabelecimentos de saúde 
estudados, para o bom atendimento das pessoas com deficiência. Isso também foi verificado por EDWARDS e MERRY (2002), que mostraram em seu estudo, realizado com dentistas em Liverpool, no Reino Unido, que os consultórios odontológicos estudados não eram totalmente acessíveis.

BEANGE (1996) relata em seu artigo que, no Canadá, a prevalência de doenças dentárias na população de pessoas com deficiência intelectual era de 86\%, e que os centros de tratamento especializado eram insuficientes. ODETTE et al. (2003) também mostraram que há maior necessidade de uso de serviços odontológicos entre pessoas com deficiência física, com menor utilização desses serviços por esses usuários. Esses estudos podem corroborar a afirmação feita por LIN et al. (2003) de que esses serviços de saúde não são adequados para o atendimento de pessoas com deficiência intelectual.

As dificuldades de acesso arquitetônico enfrentados por pessoas com deficiência no atendimento em saúde são tão importantes que, na cidade de São Paulo, a prefeitura municipal regulamentou, por meio de documento oficial, a adequação de todas as edificações públicas destinadas à saúde para, obrigatoriamente, facilitar o acesso de pessoas com deficiência ao serviço de saúde por meio de entradas e saídas facilitadas do local; mobiliário adequado; condições de circulação horizontal e vertical adequadas; largura e altura de portas e janelas; adequação de dependências de serviços; sanitários e vestiários próprios; sinalização adequada; telefones para pessoas com deficiência auditiva; bebedouros adaptados; vagas de estacionamento, entre outros (SMPED, 2005). Além disso, há o Decreto 5.296/04, de 2 de dezembro de 2004 (BRASIL, 2004), que estabelece as normas gerais e critérios para a promoção da acessibilidade. Dispomos também do conjunto de normas da Associação Brasileira de Normas Técnicas (ABNT) que tratam do tema: NBR-9050 (Acessibilidade de pessoas portadoras de deficiência ${ }^{\mathrm{C}}$ a edificações, espaço, mobiliário e equipamentos urbanos); NBR-13994 (Elevadores para transporte de pessoa portadora de deficiência); NBR-14020 (Transporte Acessibilidade à pessoa portadora de deficiência - Trem de longo percurso); NBR14021 (Transporte - Acessibilidade à pessoa portadora de deficiência - Trem

\footnotetext{
${ }^{\mathrm{C}}$ Embora não seja o mais correto, o termo "portador de deficiência" foi mantido em alguns casos por se tratar da redação original do texto
} 
metropolitano); NBR-14022 (Transporte - Acessibilidade à pessoa portadora de deficiência em ônibus e trólebus, para atendimento urbano e intermunicipal); NBR14273 (Acessibilidade da pessoa portadora de deficiência no transporte aéreo comercial); NBR 15250 (Acessibilidade em caixa de auto-atendimento bancário); NBR 15320 (Acessibilidade à pessoa com deficiência no transporte rodoviário); NBR 14022:2006 (Acessibilidade em veículos de características urbanas para o transporte coletivo); e a NBR 15450:2006 (Acessibilidade de passageiros no sistema de transporte aquaviário) ${ }^{\mathrm{D}}$.

Diante da exposição anterior, percebemos a existência de uma aparente contradição. Enquanto a pessoa com deficiência tem estados de saúde que inspiram mais cuidados de saúde que outras pessoas, ela tem, ao mesmo tempo, mais dificuldades no acesso aos serviços de saúde (SUTTON e DEJONG, 1998; KERSTEN et al., 2000; VELTMAN et al., 2001; BEATTY et al., 2003; DIAB e JOHNSTON, 2004).

Por outro lado, segundo RAVESLOOT et al. (2005), a promoção de saúde e a atenção em saúde adequadas para as pessoas com deficiência podem melhorar o estado de saúde e a sua qualidade de vida. WILLIANS e BOWIE (1993) informam que o acesso ao serviço de saúde por pessoas com deficiência física severa pode proporcionar a manutenção da independência nas tarefas diárias. A não utilização dos serviços de saúde de forma satisfatória pelas pessoas com deficiência poderia exacerbar as diferenças no perfil de saúde desses indivíduos quando comparados com outras pessoas sem a deficiência (KROLL et al., 2006).

Pela Constituição da República Federativa do Brasil, todos os cidadãos têm direito à saúde, sendo seu oferecimento um dever do Estado (BRASIL, 1988). Além disso, o Decreto 3.298 de 1999, que regulamenta a Lei 7.853, de 1989, cria a Política Nacional para a Integração da Pessoa Portadora de Deficiência, que cita a saúde como direito básico da pessoa com deficiência e ratifica o Poder Público como agente que deve assegurar o cumprimento desse preceito (BRASIL, 1999). Outro documento que garante a saúde como direito das pessoas com deficiência é a “Convenção sobre os Direitos das Pessoas com Deficiência”, que, em seu artigo 25,

\footnotetext{
${ }^{\mathrm{D}}$ http://www.mpdft.gov.br/sicorde/abnt.htm, acesso em 17/jul/2010. SICORDE, Sistema Nacional de Informação sobre Deficiência. Brasília-DF.
} 
trata do tema da saúde. Essa convenção foi proposta pela Organização das Nações Unidas (ONU) e o Brasil é um de seus signatários (BRASIL, 2007).

Entretanto, as desigualdades no acesso aos serviços de saúde são frequentes e podem variar dependendo de fatores como localização geográfica, sexo, nível socioeconômico, entre outros (WALLACE et al., 1995; CHAN et al., 1999; McALPINE e MECHANIC, 2000; CHAN et al., 2002; WITT et al., 2003). Essa variação no acesso aos serviços de saúde pode prejudicar a situação de saúde dos indivíduos. O fato de existirem barreiras entre a pessoa com deficiência e os serviços de saúde faz com que o indivíduo tenha seu direito à saúde cerceado (NERI e KROLL, 2003), o que contraria os preceitos da Constituição Brasileira e do Sistema Único de Saúde (SUS), que têm como pilares de sua política o acesso universal à saúde com equidade.

Diante de todas essas dificuldades, os relatos de insatisfação com os serviços médicos utilizados são frequentes entres as pessoas com deficiência (ROSENBACH, 1995; DEJONG, 1997; FOUTS et al., 2000; IEZZONI et al., 2002; SHAPIRO et al., 2003).

Nas últimas décadas, mais atenção tem sido dada às pessoas com deficiência e, por consequência, as oportunidades de participação social para todas as pessoas tem aumentado (IWARSSON et al., 2003). Com as mudanças no perfil demográfico e o envelhecimento da população há um aumento da prevalência de deficiências. Além disso, o aumento de ocorrências de acidentes de trânsito também tem contribuído para a ampliação do número de pessoas com deficiência na sociedade (AMERATUNGA et al., 2004; 2006).

Pelo exposto anteriormente, a questão dos serviços de saúde para as pessoas com deficiência pode ser analisada sob três pontos diferentes: o uso, o acesso e a acessibilidade. O uso seria voltado ao produto final do serviço de saúde, sua utilização completa e satisfatória pelo indivíduo. O acesso compreende a entrada, o ingresso da pessoa no serviço de saúde. A acessibilidade, por sua vez, refere-se à facilidade na obtenção ou entrada nos serviços de saúde. Assim, para o completo uso do sistema de saúde, não basta que o serviço esteja disponível e que a pessoa possa ir até ele: é necessário que estes sejam acessíveis, isto é, não apresentem barreiras ao seu uso, sejam elas de ordem física ou não. CUNHA e VIEIRA-da-SILVA (2010) 
ressaltam que a acessibilidade é um importante componente do sistema de saúde, considerando que ela efetiva o processo de busca e obtenção do atendimento.

\section{JUSTIFICATIVA}

A identificação dos problemas de acessibilidade de pessoas com deficiência aos serviços de saúde e de suas características, poderá fornecer subsídios para as políticas públicas voltadas para esse grupo, visando à concreta implantação e melhoria dessas políticas. Esses dados poderão nortear a assistência a essas pessoas, otimizando gastos e melhorando serviços.

\section{OBJETIVO}

\subsection{OBJETIVO GERAL}

Estudar os problemas de acessibilidade de pessoas com deficiência aos serviços de saúde na área dos municípios paulistas de São Paulo, Taboão da Serra, Embu e Itapecerica da Serra.

\subsection{OBJETIVOS ESPECÍFICOS}

Verificar se há variação nos problemas de acessibilidade segundo características sociodemográficas.

Estudar a ocorrência de problemas de comunicação entre pessoas com deficiência auditiva e profissionais de saúde durante o atendimento.

\section{METODOLOGIA}

\subsection{ORIGENS DO ESTUDO}

A amostra de pessoas com deficiências desta pesquisa tem origem em dois inquéritos de saúde que estão em processo de análise, sendo que alguns resultados já foram publicados, como por exemplo, o livro intitulado "SAÚDE E CONDIÇÃO DE VIDA EM SÃO PAULO - Inquérito Multicêntrico de Saúde no Estado de São Paulo - ISA-SP”' (CESAR et al., 2005); artigos científicos (FISBERG et al., 2004; AMORIM et al., 2006; FRANCISCO et al., 2006; MARAGNO et al., 2006; 
ZAITUNE et al., 2006; CASTRO et al., 2008; CARVALHO et al., 2008; BUENO et al., 2008; FRANCISCO et al., 2008, 2010; LIMA et al., 2008; TURRINI et al., 2008; MORIMOTO et al., 2008; LIMA et al., 2009a, 2009b; CRUZ et al., 2009; CAMPOS et al., 2009; FLORINDO et al., 2009; ANDRADE et al, 2010; FRANCISCO et al., 2010); teses de mestrado, doutorado e livre-docência defendidas (GUIMARÃES, 2003a, 2003b; ANDRADE, 2004; BUENO, 2004; CARVALHO, 2004; FERNANDEZ, 2004; FISBERG, 2005; GALVANESE, 2004; GODOY, 2004; PATERNEZ, 2004; MAGNABOSCO, 2005; MORIMOTO, 2005; PELICIONI, 2005; SOUZA, 2005). Foram também apresentados trabalhos em congressos e eventos e é mantido um site disponível em: <http://hygeia.fsp.usp.br/isa-

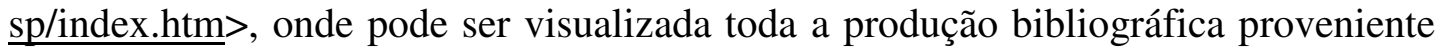
dessas pesquisas.

\subsection{DESENHO DO ESTUDO}

Esta pesquisa é um estudo de corte transversal, com dados coletados por meio de entrevistas domiciliares realizadas com pessoas com uma ou mais deficiências, moradoras dos municípios paulistas de São Paulo, Taboão da Serra, Embu e Itapecerica da Serra, no ano de 2007. O presente estudo recebeu o nome de Projeto AceSS - Acessibilidade aos Serviços de Saúde de pessoas com deficiências.

Foram estudados, na presente pesquisa, os indivíduos que auto-referiram alguma deficiência (física, auditiva ou visual) em dois inquéritos de base populacional realizados em áreas do Estado de São Paulo: o Inquérito de Saúde no Estado de São Paulo (ISA-SP - 2002), realizado em Itapecerica da Serra, Embu, Taboão da Serra, Campinas e Botucatu, e o Inquérito de Saúde no Município de São Paulo (ISA - Capital - 2003). Houve um processo de busca e localização dessas pessoas para a re-entrevista desta pesquisa.

Este estudo foi aprovado pelo Comitê de Ética em Pesquisa da Faculdade de Saúde Pública da USP (protocolo número 1653) e os entrevistadores explicaram a natureza da pesquisa aos participantes, que assinaram termo de consentimento livre e esclarecido. Os questionários e gravações telefônicas resultantes da pesquisa ficarão armazenados na Faculdade de Saúde Pública da USP e serão usados somente com 
fins de pesquisa, sendo garantida a proteção das informações de identificação dos indivíduos.

A pesquisa foi realizada em duas fases, sendo a primeira por meio da realização de entrevistas abertas. Os dados dessa fase foram analisados qualitativamente e os resultados serviram para fundamentar a criação de um questionário fechado. Esse questionário foi usado nas entrevistas da segunda fase, junto a um número maior de entrevistados.

\subsection{SUJEITOS}

A listagem de pessoas com deficiência identificadas nos dois inquéritos citados anteriormente foi a amostra inicial da presente pesquisa. Essas pessoas relataram ter algum tipo de deficiência nos inquéritos referidos anteriormente. No momento das entrevistas, foi feita a seguinte pergunta:

“O senhor(a) tem algum desses problemas?" e eram listadas as opções a seguir:

1 - Dificuldade de enxergar

2 - Cegueira de um olho

3 - Cegueira de dois olhos

4 - Dificuldade de ouvir

5 - Surdez de um ouvido

6 - Surdez de dois ouvidos

7 - Paralisia total ou parcial de membros

8 - Perda de membros ou de parte deles

9 - Outro (especificar)

10 - Não

$99-\mathrm{NS} / \mathrm{NR}$

A Figura 3 descreve o processo de obtenção da amostra final aqui estudada. 
Figura 3 - Descrição do processo de obtenção da amostra final e do total de entrevistas, Projeto AceSS, São Paulo, 2007.

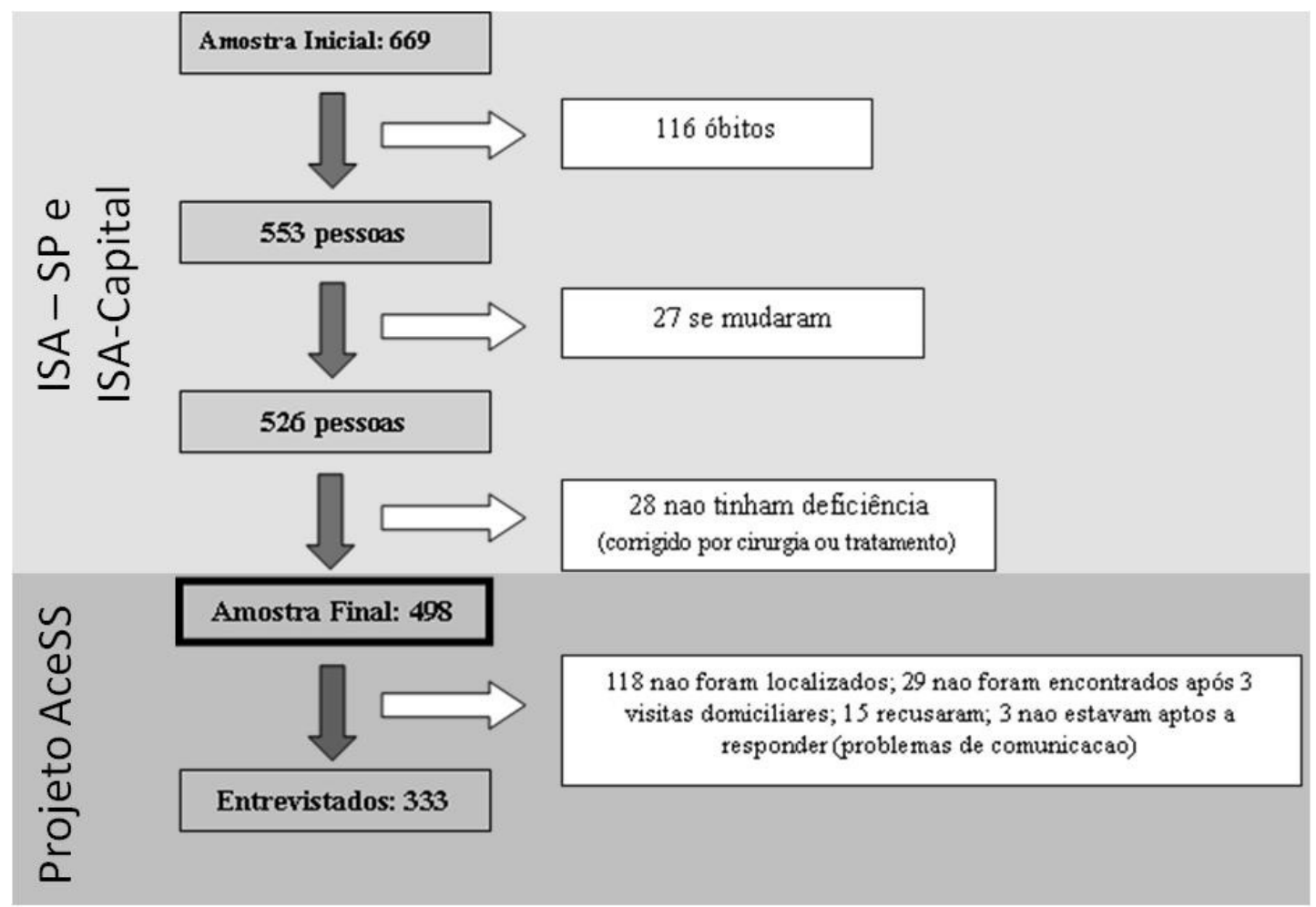

Verifica-se na Figura 3, que houve uma taxa de resposta de 66,87\%. A comparação entre a amostra final, representada pelo total de pessoas que deveriam ter sido entrevistadas, e a amostra de entrevistados mostrou diferença na composição etária dos dois grupos $\left(\mathrm{p}<0,05^{\mathrm{E}}\right)$, sendo que a amostra de entrevistados teve média de 55,75 anos (IC95\%: 53,4-58,0) e a amostra final de 63,24 anos (IC95\%: 61,6-64,8). Não houve diferenças com relação ao gênero ${ }^{\mathrm{F}}$.

\subsection{COLETA DOS DADOS}

A partir dos nomes, endereços e telefones obtidos nos questionários do ISASP e do ISA - Capital, foram destacados dois entrevistadores para trabalhar na localização e atualização dos endereços dos indivíduos a serem entrevistados. Os investigadores trabalharam antes da saída a campo da equipe de entrevistadores, de

\footnotetext{
${ }^{\text {E }}$ Significância determinada pelo teste de Wilcoxon, respeitando a não-parametricidade dos dados.

F Significância determinada pelo teste "t" de Student.
} 
forma que a coleta de campo foi iniciada depois da finalização do trabalho de investigação.

A coleta dos dados foi feita por entrevistadores treinados para a aplicação do questionário da pesquisa, que foi especificamente elaborado para este estudo. $\mathrm{O}$ treinamento teve o objetivo de padronizar os procedimentos de coleta de dados empregados na pesquisa. Eles foram treinados com entrevistas aplicadas entre si e em terceiros, que reportaram depois, aos coordenadores, suas opiniões a respeito do entrevistador e sobre sua maneira de aplicação dos questionários. Houve uma verificação de $10 \%$ dos questionários para controle de qualidade do preenchimento e do trabalho dos entrevistadores.

As entrevistas foram realizadas com as pessoas com deficiência ou com seus cuidadores; com familiares ou pessoas mais próximas da pessoa com deficiência, caso a mesma não pudesse responder sem auxílio. Essa abordagem, onde uma pessoa próxima ("proxy”) responde por outra, já foi estudada e tem sua eficácia comprovada (CUSICK et al., 2001).

A existência de um banco de dados sobre as pessoas com deficiência facilitou o desenvolvimento do presente estudo uma vez que disponibilizou para os pesquisadores a lista com todas as informações necessárias sobre todas as pessoas com deficiência entrevistadas nos inquéritos ISA-SP e ISA - Capital. Além disso, esses inquéritos já haviam coletado informações referentes às características socioeconômicas (sexo, idade, escolaridade, estado conjugal, renda, entre outras), demográficas (número de pessoas da família, situação de domicílio e outras) e referentes ao uso de serviços de saúde (hospitalização, uso de medicamentos, uso de serviços odontológicos e outras) e sua cobertura entre as pessoas com deficiência (maiores informações no Quadro 2, a seguir, com a descrição dos temas já abordados pelo ISA-SP e ISA - Capital. 
Quadro 2 - Conteúdo temático dos blocos dos questionários, segundo pesquisa, São Paulo, 2007.

\begin{tabular}{|c|c|c|c|}
\hline Bloco & Assunto & $\begin{array}{l}\text { ISA- } \\
\text { SP }\end{array}$ & $\begin{array}{c}\text { ISA - } \\
\text { Capital }\end{array}$ \\
\hline A & Composição familiar & $\mathrm{X}$ & $\mathrm{X}$ \\
\hline B & Controle de visitas, identificação do entrevistado & $\mathrm{X}$ & $\mathrm{X}$ \\
\hline $\mathrm{C}$ & Morbidade referida nos últimos 15 dias & $\mathrm{X}$ & $\mathrm{X}$ \\
\hline $\mathrm{D}$ & Doenças crônicas & $\mathrm{X}$ & $\mathrm{X}$ \\
\hline $\mathrm{E}$ & Deficiências & $\mathrm{X}$ & $\mathrm{X}$ \\
\hline $\mathrm{F}$ & Acidentes e violências & $\mathrm{X}$ & $\mathrm{X}$ \\
\hline G & Utilização de serviços de saúde & $\mathrm{X}$ & $\mathrm{X}$ \\
\hline $\mathrm{H}$ & Exames preventivos & $\mathrm{X}$ & $\mathrm{X}$ \\
\hline I & Cobertura vacinal e informações sobre a dengue & $\mathrm{X}$ & $\mathrm{X}$ \\
\hline $\mathbf{J}$ & Consumo de medicamentos & $\mathrm{X}$ & $\mathrm{X}$ \\
\hline $\mathrm{K}$ & Hospitalizações nos últimos 12 meses & $\mathrm{X}$ & $\mathrm{X}$ \\
\hline $\mathrm{L}$ & $\begin{array}{l}\text { Estilo de vida (hábitos alimentares, atividades } \\
\text { físicas, fumo e álcool) }\end{array}$ & $\mathrm{X}$ & $\mathrm{X}$ \\
\hline M & Saúde emocional & $\mathrm{X}$ & $\mathrm{X}$ \\
\hline $\mathrm{N}$ & Qualidade de vida & $\mathrm{X}$ & $\mathrm{X}$ \\
\hline $\mathrm{O}$ & Caracterização socioeconômica do entrevistado & $\mathrm{X}$ & $\mathrm{X}$ \\
\hline $\mathrm{P}$ & Caracterização da família e do domicílio & $\mathrm{X}$ & $\mathrm{X}$ \\
\hline Q & $\begin{array}{l}\text { Caracterização socioeconômica do chefe da } \\
\text { família }\end{array}$ & $\mathrm{X}$ & $\mathrm{X}$ \\
\hline $\mathrm{R}$ & Gasto familiar mensal com saúde & $\mathrm{X}$ & $\mathrm{X}$ \\
\hline $\mathrm{S}$ & Saúde materno infantil & $\mathrm{X}$ & $\mathrm{X}$ \\
\hline $\mathrm{T}$ & Programa Saúde da Família & & $\mathrm{X}$ \\
\hline $\mathrm{V}$ & Informações sobre presença de animais & & $\mathrm{X}$ \\
\hline
\end{tabular}

A coleta dos dados foi executada em duas fases distintas, com diferentes objetivos em cada uma delas conforme detalhado a seguir.

\subsubsection{Fase I}

Considerando que estudos com o mesmo enfoque e que possuem um questionário de coleta de dados específico sobre os problemas de acessibilidade aos serviços de saúde de pessoas com deficiência são escassos, houve alguma dificuldade para encontrar um instrumento de coleta de dados adequado. Devido a esse fato, definimos a estratégia de criar um questionário específico para esta pesquisa. Esse questionário final seria composto por questões fechadas. Entretanto, como fase prévia (Fase I), um instrumento de coleta de dados por entrevista foi montado com pergunta abertas. Esse primeiro questionário foi aplicado em uma amostra 
intencional de pessoas com deficiências. A amostra foi composta por 25 indivíduos da cidade de São Paulo, que já faziam parte da amostra total. A descrição desse grupo inicial de entrevistados quanto à deficiência, sexo e idade pode ser verificada na Tabela 1.

Tabela 1 - Distribuição dos indivíduos entrevistados na Fase I segundo deficiência, sexo e média de idade, Projeto AceSS, São Paulo, 2007.

\begin{tabular}{|c|c|c|c|c|}
\hline \multirow[t]{2}{*}{ Deficiência } & \multirow[t]{2}{*}{ Idade média } & \multicolumn{2}{|c|}{ Sexo } & \multirow[t]{2}{*}{ Total } \\
\hline & & Masculino & Feminino & \\
\hline Dificuldade visual & 58,25 & 4 & 4 & 8 \\
\hline Cegueira de um olho & 61,33 & 1 & 2 & 3 \\
\hline Dificuldade de ouvir & 45,0 & 3 & 2 & 5 \\
\hline Surdez de um ouvido & 64,5 & 1 & 1 & 2 \\
\hline Perda ou paralisia de membros & 62,28 & 3 & 4 & 7 \\
\hline Total & 58,27 & 12 & 13 & 25 \\
\hline
\end{tabular}

O questionário montado para essa etapa (Anexo I) foi confeccionado após reuniões e entrevistas com profissionais da Secretaria Municipal da Pessoa com Deficiência e Mobilidade Reduzida de São Paulo (SMPED, 2005) para delineamento dos temas a serem abordados, com o objetivo de aproveitar a experiência de profissionais com deficiência que trabalham na secretaria na área de acessibilidade.

O principal objetivo da Fase I deste estudo foi determinar as queixas mais frequentes entre as pessoas com deficiência no que se refere às dificuldades na acessibilidade aos serviços de saúde.

\subsubsection{Fase II}

Durante a Fase II do Projeto AceSS foram entrevistadas, em domicílio, 333 pessoas com algum tipo de deficiência. O principal objetivo da Fase II da pesquisa foi obter os dados quantitativos necessários para a resposta das questões do estudo. A descrição das características sociodemográficas desse grupo é apresentada na Tabela 2: 
Tabela 2 - Características sociodemográficas da amostra de sujeitos estudados, Projeto AceSS, São Paulo, 2007.

\begin{tabular}{|c|c|c|}
\hline Variáveis & $\mathrm{n}(\mathbf{3 3 3})$ & $\%(100.00)$ \\
\hline \multicolumn{3}{|l|}{ Deficiência } \\
\hline Visual & 137 & 41,14 \\
\hline Auditiva & 96 & 28,82 \\
\hline Paralisia ou Amputação & 26 & 7,82 \\
\hline Múltipla & 74 & 22,22 \\
\hline \multicolumn{3}{|l|}{ Sexo } \\
\hline $\mathbf{M}$ & 162 & 48,64 \\
\hline $\mathbf{F}$ & 171 & 51,36 \\
\hline \multicolumn{3}{|l|}{ Idade (anos) } \\
\hline 14-54 & 86 & 25,82 \\
\hline $55-69$ & 87 & 26,12 \\
\hline 70-77 & 81 & 24,33 \\
\hline 78-97 & 79 & 23,73 \\
\hline \multicolumn{3}{|l|}{ Renda do chefe da família ${ }^{(1)}$} \\
\hline$<1$ & 205 & 61,56 \\
\hline 1 & 39 & 11,71 \\
\hline 2-4.99 & 46 & 13,81 \\
\hline $5-7.99$ & 21 & 6,30 \\
\hline$>8$ & 22 & 6,61 \\
\hline \multicolumn{3}{|l|}{ Raça } \\
\hline Branco & 197 & 59,16 \\
\hline Não branco & 136 & 40,84 \\
\hline \multicolumn{3}{|c|}{$\begin{array}{l}\text { Necessidade de ajuda nas atividades de vida } \\
\text { diária }^{(2)}\end{array}$} \\
\hline Não & 296 & 88,89 \\
\hline Sim & 37 & 11,11 \\
\hline \multicolumn{3}{|c|}{$\begin{array}{l}\text { Necessidade de ajuda para ir ao serviço de } \\
\text { saúde por causa da deficiência } \\
\text { (3) }\end{array}$} \\
\hline Não & 260 & 79,03 \\
\hline Sim & 69 & 20,97 \\
\hline
\end{tabular}

Notas:

(1) Salários mínimos à época da entrevista

(2) Ajuda para tomar banho, vestir-se, comer, levantar e/ou caminhar

${ }^{(3)}$ Foi considerado o último serviço de saúde usado

O instrumento de coleta dos dados utilizado na Fase II foi um questionário fechado (Anexo II), contendo diferentes blocos, como explicado no Quadro 3. 


\section{Quadro 3 - Composição do questionário fechado do estudo AceSS, suas variáveis, temas, número de questões e especificidades segundo tipo de deficiência, 2008.}

\begin{tabular}{|c|c|c|c|c|c|}
\hline \multirow[t]{2}{*}{ Bloco } & \multirow[t]{2}{*}{ Assunto } & \multirow{2}{*}{$\begin{array}{l}\text { Número de } \\
\text { questões }\end{array}$} & \multicolumn{3}{|c|}{ Deficiência } \\
\hline & & & Visual & Auditiva & Física \\
\hline $\mathbf{A}$ & Identificação do entrevistado & 29 & $\mathrm{X}$ & $\bar{X}$ & $\mathrm{X}$ \\
\hline B1 & $\begin{array}{l}\text { Usos de serviço de saúde - acessibilidade - } \\
\text { (pessoas com deficiência visual ou auditiva) }\end{array}$ & 14 & $\mathrm{X}$ & $X$ & \\
\hline B2 & $\begin{array}{l}\text { Usos de serviço de saúde - acessibilidade } \\
\text { (pessoas com deficiência física) }\end{array}$ & 33 & & & $\mathrm{X}$ \\
\hline C & Exames e orientações em saúde & 41 & $\mathrm{X}$ & $\mathrm{X}$ & $\mathrm{X}$ \\
\hline D & Doenças crônicas & 15 & $\mathrm{X}$ & $\mathrm{X}$ & $\mathrm{X}$ \\
\hline E1 & Exames auditivos & 04 & & $\mathrm{X}$ & \\
\hline E2 & Exames visuais & 04 & $\mathrm{X}$ & & \\
\hline $\mathbf{F}$ & Comunicação e atendimento & 11 & & $\mathrm{X}$ & \\
\hline G & Necessidade de saúde não suprida & 14 & $\mathrm{X}$ & $\mathrm{X}$ & $\mathrm{X}$ \\
\hline $\mathbf{H}$ & Programa de Saúde da Família & 03 & $\mathrm{X}$ & $\mathrm{X}$ & $\mathrm{X}$ \\
\hline I & $\begin{array}{c}\text { Próteses/órteses e outros } \\
\text { equipamentos/insumos (pessoas com } \\
\text { deficiência auditiva) }\end{array}$ & 06 & & $\mathrm{X}$ & \\
\hline $\mathbf{J}$ & $\begin{array}{c}\text { Próteses/órteses e outros } \\
\text { equipamentos/insumos (pessoas com } \\
\text { deficiência visual) }\end{array}$ & 05 & $\mathrm{X}$ & & \\
\hline $\mathbf{K}$ & $\begin{array}{c}\text { Próteses/órteses e outros } \\
\text { equipamentos/insumos (pessoas com } \\
\text { deficiência física) }\end{array}$ & 09 & & & $\mathrm{X}$ \\
\hline Total & & 188 & 125 & 137 & 144 \\
\hline
\end{tabular}

Procurou-se, durante a construção do questionário da Fase II, contemplar as principais respostas obtidas na análise da Fase I, além de adicionar outros itens pesquisados na literatura. Alguns conceitos sobre fatores ambientais contidos na Classificação Internacional de Funcionalidade, Incapacidade e Saúde (CIF) também serviram de orientação na criação desse questionário. Durante a montagem das perguntas, o marco teórico foi voltado às concepções de interação entre corpo e ambiente apresentados pela CIF.

$\mathrm{Na}$ Fase II, os entrevistadores foram a campo para a realização das entrevistas domiciliares portando um crachá de identificação, material explicativo sobre a pesquisa, no qual constavam os nomes e os contatos dos responsáveis pelo estudo, questionários de coleta de dados, manual do questionário para consulta de dúvidas e a cartilha Guia de Encaminhamentos para pessoas com deficiência e mobilidade reduzida ${ }^{\mathrm{G}}$ (Anexo IV). Foi distribuído também às pessoas entrevistadas material de

\footnotetext{
${ }^{\mathrm{G}}$ Material elaborado por um Grupo de Trabalho Intersecretarial da Prefeitura da Cidade de São Paulo, formado pela secretarias de Assistência e Desenvolvimento Social - SMADS, de Cultura - SMC, de
} 
divulgação do Conselho Municipal da Pessoa com Deficiência $\left(\mathrm{CMPD}^{\mathrm{H}}\right.$ ) (Anexo III). Os prospectos foram obtidos junto ao próprio conselho, que os disponibilizou após uma explicação sobre a pesquisa e sobre as pessoas a serem entrevistadas.

Os dados obtidos nessa fase foram digitados em um banco de dados utilizando-se o software EpiData. A consistência dos dados foi verificada por meio de rotinas elaboradas com o auxílio do programa computacional Stata 9.2®.

\subsection{ANÁLISE DOS DADOS}

Como a pesquisa teve duas fases distintas de coleta de dados, usando metodologias diversas entre si, foram necessários métodos de análise diferentes para cada uma das fases, como detalhado a seguir.

\subsubsection{Fase I}

Os dados coletados nessa fase foram obtidos por entrevistas abertas, originando assim, informações de natureza qualitativa. Para análise do material foi utilizada metodologia do Discurso do Sujeito Coletivo (DSC) idealizada por LEFEVRE e LEFEVRE (2003; 2006). Segundo os autores, esse método é

“uma forma não-matemática nem metalinguística de representar (e de produzir), de modo rigoroso, o pensamento de uma coletividade, o que se faz mediante uma série de operações sobre os depoimentos, que culmina em discursossíntese que reúnem respostas de diferentes indivíduos, com conteúdos discursivos de sentido semelhante" (LEFEVRE e LEFEVRE, 2005).

Educação - SME, de Esporte, Lazer e Recreação - SEME, de Saúde - SMS, do Trabalho - SMTRAB, de Transportes - SMT, de Participação e Parceria - SMPP, e coordenado pela Secretaria Municipal da Pessoa com Deficiência e Mobilidade Reduzida - SMPED. O guia traz informações sobre serviços de assistência e desenvolvimento social; cultura; educação; esporte, lazer e educação; saúde; trabalho e transporte. Os entrevistadores foram orientados a esclarecer possíveis dúvidas sobre os serviços de saúde que a pessoa com deficiência pode utilizar dentro do município de São Paulo, agilizando assim, seu atendimento. O referido guia está disponível em:

$<$ http://ww2.prefeitura.sp.gov.br/arquivos/secretarias/deficiencia_mobilidade_reduzida/servicos/0008/ Guia_Encaminhamentos.pdf $>$ Acessado em 20 jul. 2010

${ }^{\mathrm{H}} \mathrm{O}$ CMPD trabalha com alguns temas, entre eles: acesso aos transportes coletivos; educação para todos; serviços de saúde para as pessoas com deficiências nos territórios das sub-prefeituras; acessibilidade às edificações públicas e de uso público, calçadas e parques; entre outros. 
O DSC ajuda na determinação das questões levantadas em cada resposta na medida em que sua metodologia prevê que o discurso de cada respondente tenha os conceitos-chave extraídos para uma posterior categorização das ideias centrais, que podem se repetir nas respostas de mais de um entrevistado para a mesma pergunta. Depois disso, as ideias centrais de todos os entrevistados nas mesmas perguntas são categorizadas e agrupadas. A partir desse procedimento é confeccionado o DSC (LEFEVRE e LEFEVRE, 2006). Neste estudo, o DSC não foi elaborado, pois as idéias centrais categorizadas, na fase imediatamente anterior à formação do DSC suprem a necessidade de se obter as opções de respostas dos entrevistados.

O software Qualiquantsof®, criado pelos mesmos autores do DSC tem a finalidade de viabilizar pesquisas que usem o DSC, possibilitando maior agilidade e confiabilidade metodológica ao estudo (LEFEVRE e LEFEVRE, 2005).

Outros detalhes sobre a análise da Fase I da pesquisa podem ser visualizados no Manuscrito 1, na seção de Resultados e Discussão, na qual o DSC foi usado para análise e discussão do material.

\subsubsection{Fase II}

Os dados da Fase II foram analisados quantitativamente. Foram calculadas frequências e porcentagens e a sua relação com variáveis sociodemográficas foi testada usando-se o teste de Qui-Quadrado. O teste de Qui-Quadrado de tendência foi executado quando a variável tinha mais que duas categorias e uma tendência linear foi percebida.

Modelos de Poisson com variância robusta foram usados para a determinação da Razão de Prevalência (RP), uma vez que a prevalência estudada foi menor que $20 \%$ (BARROS e HIRAKATA; 2003), e considerando que os intervalos de confiança encontrados com o uso da variância robusta são mais indicados, adequando-se melhor a pequenos números (COUTINHO et al., 2008). O método de seleção do melhor modelo foi o stepwise backward selection, em que todas as variáveis são adicionadas ao modelo inicial e posteriormente, retiradas do mesmo até que se chegue ao modelo final, sendo que as variáveis com significância estatística maior que 0,20 foram progressivamente retiradas do modelo. Outras variáveis, que 
mostraram significância estatística entre 0,20 e 0,05 e alteravam os coeficientes do modelo, foram mantidas para efeitos de ajuste. A significância estatística adotada foi de $5 \%$ e as análises foram executadas com a ajuda do programa Stata 9.2®. 


\section{RESULTADOS E DISCUSSAO}

\subsection{MANUSCRITO 1}

Artigo aceito para publicação no periódico Revista de Saúde Pública.

Acessibilidade aos serviços de saúde por pessoas com deficiência

Accessibility to the health services by people with disability. Qualitative research.

Shamyr Sulyvan Castro1

Fernando Lefèvre 2

Ana Maria Cavalcanti Lefèvre 3

Chester Luiz Galvão Cesar2

1 - Doutorando em Saúde Pública - Departamento de Epidemiologia - Faculdade de Saúde Pública. Universidade de São Paulo. São Paulo, SP, Brasil

2 - Departamento de Prática de Saúde - Faculdade de Saúde Pública. Universidade de São Paulo. São Paulo, SP, Brasil

3 - Instituto de Pesquisa do Discurso do Sujeito Coletivo - IPDSC. São Paulo, SP, Brasil.

4 - Departamento de Epidemiologia - Faculdade de Saúde Pública. Universidade de São Paulo. São Paulo, SP, Brasil

Correspondência I Correspondence:

Shamyr Sulyvan Castro

Faculdade de Saúde Pública - FSP

Departamento de Epidemiologia

Av. Dr. Arnaldo, 715 - Cerqueira César

01246-904 São Paulo, SP, Brasil

E-mail: shamyr@usp.br

Recebido: $2 / 3 / 2010$

Aprovado: 23/5/2010 


\section{RESUMO}

Objetivo. Analisar as dificuldades de acessibilidade aos serviços de saúde vividas por pessoas com deficiência.

Procedimentos metodológicos: Estudo qualitativo realizado com pessoas que relataram ter algum tipo de deficiência (paralisia ou amputação de membros; baixa visão, cegueira unilateral ou total; baixa audição, surdez unilateral ou total). Foram entrevistados 25 indivíduos (14 mulheres) na cidade de São Paulo, SP, de junho a agosto de 2007, que responderam perguntas referentes à deslocamento a acessibilidade aos serviços de saúde. A metodologia utilizada para análise foi o discurso do sujeito coletivo e as análises foram conduzidas com recurso ao programa Qualiquantsoft.

Análise dos Resultados: A análise dos discursos sobre o deslocamento ao serviço de saúde mostrou diversidade quanto ao usuário ir ao serviço sozinho ou acompanhado, utilizar carro particular, transporte coletivo, ir a pé ou de ambulância e demandar tempo variado para chegar ao serviço. Com relação às dificuldades oferecidas de acessibilidade pelos serviços de saúde, houve relatos de demora no atendimento, problemas com estacionamento, falta de rampas, elevadores, cadeiras de rodas, sanitários adaptados e de médicos.

Conclusões. As pessoas com algum tipo de deficiência fizeram uso de meios de transporte diversificados, necessitando de companhia em alguns casos. Problemas na acessibilidade dos serviços de saúde foram relatados pelos sujeitos com deficiências, contrariando o princípio da eqüidade, preceito do Sistema Único de Saúde.

Descritores: Pessoas com Deficiência. Limitação da Mobilidade. Acesso aos Serviços de Saúde. Saúde da Pessoa com Deficiência ou Incapacidade. Pesquisa Qualitativa.

Descriptores: Personas con Discapacidad. Limitación de la Movilidad. Accesibilidad a los Servicios de Salud. Salud de la Persona con Deficiencia o Discapacidad. Investigación Cualitativa. 
Objective:To describe the difficulties faced by the people with disabilities in the accessibility of the health services.

Methods. Qualitative research carried out with people with some type of disability (paralysis or amputation of members; low vision, unilateral or total blindness; low hearing, unilateral or total deafness). 25 persons (14 women) were interviewed from June to August, 2007, and answered the questions: "How do you (Mr. or Ms.) do to go to the health service?....Talk more about this..." and "Many health services offer difficulties for the people with disability as lack of slopes, car parking, signaling, etc...And for you (Mr. or Ms.), how has it been?". To study the collective discourses, the analysis was made with the "QualiQuantSoft" software and with the methodology of the "Discourse of the Collective Subject".

Analysis of the Discourses. Regarding to the displacement until the health service, the answers of the interviewed had shown diversity: alone or with company; particular car, collective transport, walking or ambulance; and variable time to arrive at the service. Concerning to the difficulties offered for the health services, there were accounts of delay in the attendance; problems with parking; lack of slopes, elevators, wheelchairs, adapted sanitary, and physicians.

Conclusions. The people with disability make use of diverse types of transport, needing company in some cases. Problems in the accessibility of the health services were mentioned by the citizens with disability, opposing the equity, a precept of the Unified Health System (SUS).

Descriptors: Disabled Persons. Mobility Limitation. Health Services Accessibility. Disabled Health. Qualitative Research. 


\section{Introdução}

Indivíduos com deficiência estão mais expostos a comorbidades associadas à sua

deficiência, ${ }^{10,18}$ resultando em maior necessidade de uso de serviços de saúde para a manutenção de sua integridade física e mental.

Entretanto, entre a necessidade de serviços e sua satisfação há a questão da acessibilidade aos serviços, que se não for adequadamente trabalhada pode fazer com que o deficiente enfrente obstáculos que inviabilizem o seu acesso aos serviços de saúde. A acessibilidade é definida por Frenk ${ }^{8}$ (1985) como o produto da relação entre a disponibilidade efetiva de serviços de saúde e o acesso por parte dos indivíduos a esses serviços. A disponibilidade efetiva acontece quando se analisa a disponibilidade dos serviços de saúde frente à resistência que o meio proporciona a essa disponibilidade. ${ }^{8}$ Essa resistência tem especial importância quando se estuda a acessibilidade de pessoas com deficiência aos serviços de saúde. Ela pode ser entendida como a dificuldade encontrada na obtenção dos serviços de saúde e é determinante para a saúde das pessoas com deficiência. Em virtude do quadro geral da deficiência e das co-morbidades dele advindas, mais dificuldades se interpõem entre a pessoa com deficiência e o serviço de saúde, aumentando assim a resistência. $^{25}$

Como a ocorrência de deficiências tem relação direta com o aumento da longevidade populacional e com o incremento de ocorrências por causas externas, ${ }^{5}$ a porcentagem de pessoas com deficiência na comunidade tende a aumentar com o passar do tempo. Sendo assim, investigar as dificuldades encontradas pelas pessoas com deficiências na acessibilidade aos serviços de saúde pode fornecer subsídios para o planejamento em saúde, estruturando e melhorando os serviços de forma que atendam a esse grupo populacional de maneira adequada.

O objetivo do presente estudo foi analisar as dificuldades de acessibilidade aos serviços de saúde vividas por pessoas com deficiência. 


\section{Procedimentos Metodológicos}

Pesquisa qualitativa de natureza exploratória realizada com amostra intencional de 25 indivíduos com algum tipo de deficiência (visual, auditiva ou física). A identificação das pessoas a serem entrevistadas foi obtida de uma listagem maior, com 414 sujeitos com deficiências, provenientes do total de pessoas entrevistadas pelo Inquérito de Saúde e Condições de Vida no Município de São Paulo (ISA Capital) $-2003 .^{1}$

O estudo integrou a fase inicial do Projeto Acessibilidade aos Serviços de Saúde (AceSS), realizado com 333 pessoas com algum tipo de deficiência em na capital São Paulo e em uma área da grande São Paulo (Embu, Taboão da Serra e Itapecerica da Serra) no ano de 2007. As entrevistas foram realizadas de junho a agosto de 2007 com um questionário semi-estruturado a respeito das dificuldades na acessibilidade aos serviços de saúde pelas pessoas com algum tipo de deficiência. Foram analisadas questões sobre o deslocamento do deficiente ao serviço de saúde "Como o(a) senhor(a) faz para ir até o serviço de saúde?... Fale um pouco mais sobre isso..." e sobre os problemas de acessibilidade aos serviços de saúde ("Muitos serviços de saúde oferecem dificuldades para as pessoas com deficiência como falta de rampas, estacionamentos, sinalização, etc.. E para o(a) senhor(a), como tem sido isso?”.

A coleta dos dados ocorreu por entrevistas domiciliares que foram gravadas e transcritas integralmente. Com as informações obtidas na transcrição das conversas foi criado um banco de dados, o qual foi analisado pelo software QualiQuantSoft?. ${ }^{12}$ Este programa tem o objetivo de facilitar a realização de pesquisas que empreguem o Discurso do Sujeito Coletivo (DSC) como metodologia. O QualiQuantiSoft permite associar pensamentos, crenças, valores, representações, às características objetivas dos portadores destas representações, tais como sexo, idade, grau de instrução, renda

\footnotetext{
${ }^{1}$ http://hygeia.fsp.usp.br/isa-sp/ - Cesar CLG, Carandina L, Alves MCGP, Barros MBA, Goldbaum M. SAÚDE E CONDIÇÃO DE VIDA EM SÃO PAULO - Inquérito Multicêntrico de Saúde no Estado de São Paulo - ISA-SP. 2005
} 
e outras. ${ }^{2} \mathrm{O}$ software cria um ambiente virtual que guia o pesquisador no uso do DSC como instrumento de análise de estudos qualitativos, contemplando e auxiliando em todas as etapas do método de forma clara e dirigida. Ele se configura dessa forma, em um instrumento de execução do DSC, englobando assim, essa metodologia em sua amplitude total e orientando-se estritamente por ela.

A partir do banco de dados foram extraídas as idéias centrais das respostas de cada indivíduo e, posteriormente, construídos os DSC, tendo como objetivo expressar empiricamente o pensamento do grupo de entrevistados, como um conjunto, não como ser individual, ${ }^{12}$ criando assim um ente pensante coletivo, com voz e respostas às perguntas efetuadas.

A metodologia de pesquisa qualitativa avalia a percepção dos indivíduos, sendo concebida a partir do produto dos discursos/verbalizações de atores sociais diretamente envolvidos com o assunto. ${ }^{17} \mathrm{~A}$ abordagem qualitativa do modo de pensar as questões estudadas permite que nos apossemos das percepções individuais dos entrevistados e as transformemos em um discurso mais coeso e consistente, podendo assim, obter as representações sociais referentes aos temas trabalhados neste grupo de indivíduos. A metodologia qualitativa é usada no campo de estudos das deficiências. ${ }^{6,11,21}$

Segundo Nagai et $\mathrm{al}^{17}$ (2007), o DSC pode ter mais de uma idéia central na resposta de um indivíduo ou a mesma idéia central pode emergir dos discursos de muitos sujeitos diferentes. Existe ainda a possibilidade de que algumas dessas falas tenham identidade própria e não reflitam a resposta de outras pessoas. Lèfreve et $\mathrm{al}^{13}$ (2006) esclarece ainda que desse modo, as respostas podem abordar de maneiras diversas um mesmo problema, complementando-se umas às outras e/ou explicando-se mutuamente.

As idéias centrais de cada pergunta foram descritas em itens e foi construído um DSC para cada uma delas.

\footnotetext{
${ }^{2}$ Lèfevre F, Lèfevre AMC, Simioni AAC, Ferraz MTMA. Acessando o Discurso do Sujeito Coletivo e o software QualiQuantiSoft. Manual. www.ipdsc.com.br, acessado em 08/09/2009.
} 
Das 25 pessoas com deficiência entrevistadas, três tinham idades entre 11 e 20 anos (três mulheres); três entre 31 e 40 anos (duas mulheres); 12 tinham de 55 a 69 anos (quatro mulheres); e sete de 70 a 90 anos (cinco mulheres).

Entre os entrevistados, oito indivíduos relataram ter algum tipo de deficiência física (amputação ou paralisia de membros); nove indivíduos auto-referiram deficiência auditiva (diminuição da audição ou surdez unilateral) e oito indivíduos relataram deficiência visual (diminuição da visão ou cegueira unilateral). Os participantes eram usuários tanto de serviços do Sistema Único de Saúde (SUS) quanto de serviços de convênios de empresas privadas.

O estudo foi aprovado pelo Comitê de Ética em Pesquisa da Faculdade de Saúde Pública da USP (Prot. N. 1653/2007). Todos os participantes assinaram termo de consentimento livre e esclarecido. 


\section{ANÁLISE DOS RESULTADOS e DISCUSSÃO}

\section{Deslocamento ao serviço de saúde}

As idéias centrais do discurso do sujeito coletivo referentes ao deslocamento ao serviço de saúde (Questão 1- Como o(a) senhor(a) faz para ir até o serviço de saúde? Fale um pouco mais sobre isso) foram:

Idéia central 1.1: Vai sozinho, usa transporte coletivo e é rápido para chegar [DSC 1.1] Eu vou de transporte coletivo; normalmente não preciso de ninguém para me acompanhar e não demora para chegar porque é perto.

Idéia central 1.2: Vai acompanhado, usa carro particular e demora para chegar [DSC 1.2] Tenho que ir acompanhado, uso carro particular e não demora para chegar.

Idéia central 1.3: Vai acompanhado, usa transporte coletivo demora para chegar

[DSC 1.3] Vou acompanhado, uso transporte coletivo e demora para chegar ao serviço de saúde.

Idéia central 1.4: Vai acompanhado e de ambulância

[DSC 1.4] Tenho que ir de ambulância porque não posso me sentar e tenho que ter companhia; é difícil vir médico na minha casa.

Idéia central 1.5: Vai acompanhado, usa transporte coletivo e não demora para chegar

[DSC 1.5] Vou de ônibus ou metrô, preciso de companhia e o serviço e saúde fica perto da minha casa

Idéia central 1.6: Vai sozinho, usa transporte coletivo e demora para chegar

[DSC 1.6] Vou sozinho ao serviço de saúde, só se precisar levo alguém; uso transporte coletivo e demoro para chegar ao serviço de saúde. 
Idéia central 1.7: Vai sozinho, de carro particular e demora para chegar

[DSC 1.7] Vou de carro, posso ir sozinho e demora para chegar porque o serviço de saúde não é muito perto.

Idéia central 1.8: Vai acompanhado, a pé e não demora para chegar

[DSC 1.8] Vou a pé porque é perto, não demora; e geralmente vou acompanhado.

Idéia central 1.9: Vai acompanhado, de carro particular e demora para chegar

[DSC 1.9] Tenho sempre que ir acompanhado, para levar tem que ser de carro e demora para chegar porque é longe ou tem trânsito.

Os discursos relatados mostraram diversidade de opiniões ente os entrevistados, com variações quanto ao tempo de deslocamento, meio de transporte utilizado e necessidade de companhia. Quando se analisa o tempo gasto observa-se que aproximadamente um terço dos respondentes apontaram essa dificuldade em seu deslocamento para o serviço de saúde. Travassos et al. ${ }^{23}$ (2004) afirmam que a acessibilidade geográfica é um importante fator para o efetivo uso dos serviços de saúde, podendo atenuar ou ampliar as dificuldades no acesso. No artigo citado anteriormente, as autoras sugerem que a correta distribuição espacial dos serviços de saúde e dos pacientes deve ser coerente para o uso adequado.

De acordo com os discursos, aproximadamente metade das pessoas com deficiência relataram ter necessidade de acompanhantes. Caldas ${ }^{2}$ (2003) discute a dependência familiar por parte de idosos e ressalta a ligação entre a dependência e a fragilidade, mostrando uma estreita relação entre o suporte oferecido ao idoso e a execução das suas tarefas diárias. Este exemplo pode ser aplicado às pessoas com deficiências, que de certa forma também têm características de fragilidade em virtude do processo incapacitante. ${ }^{9}$ Para Othero \& Dalmaso ${ }^{20}$ (2009), a família ou o cuidador tem importante papel na saúde da pessoa com deficiência no que diz respeito ao contato do cuidador/família com os profissionais de saúde, recebendo instruções que complementem o tratamento da pessoa com deficiência. Em estudo realizado na 
cidade de São Paulo, a deficiência aliada à dependência tem se mostrado freqüente com o aumento da longevidade e da ocorrência de incapacidades. ${ }^{3}$

A respeito do meio de locomoção usado pelas pessoas com deficiências, os discursos mostram padrões variados. Aproximadamente a metade dos entrevistados fez o percurso utilizando meios de transporte coletivos. Araújo et $\mathrm{al}^{1}$ (2006) observam a importância do fator transporte para a estrutura familiar no que diz respeito a gastos e qualidade em saúde, implicando dispêndio financeiro e dificuldades no uso dos serviços de saúde.

Observou-se ainda referência à necessidade de uso de ambulância para o deslocamento ao serviço de saúde. Isto se mostra um obstáculo, uma vez que o paciente depende da disponibilidade do referido meio de transporte para sua locomoção até o estabelecimento de saúde, considerando que algumas pessoas com deficiência necessitam deste tipo de transporte por terem sua funcionalidade muito comprometida, inviabilizando o uso de outros meios de transporte.

\section{Acessibilidade aos serviços de saúde}

As idéias centrais do discurso do sujeito coletivo referentes à acessibilidade aos serviços de saúde (Questão 2 - Muitos serviços de saúde oferecem dificuldades para as pessoas com deficiência como falta de rampas, estacionamentos, sinalização, etc.. E para o(a) senhor(a), como tem sido isso?) foram:

Idéia central 2.1: Sem problemas

[DSC 2.1] Eu sempre fui bem atendido, tem estacionamento, rampa, corrimão, elevador, sinalização, banheiro especial e sala de espera.

Idéia central 2.2: Demora no atendimento

[DSC 2.2] Há uma certa demora no atendimento porque sempre tem uma fila de espera. Se marcam para as $10 \mathrm{~h}$ tem que chegar às $6 \mathrm{~h}$; tem que chegar bem cedo.

Idéia central 2.3: Problemas com estacionamento 
[DSC 2.3] Não tem estacionamento, então tem que parar na rua mesmo

Idéia central 2.4: Falta de rampas

[DSC 2.4] Não tem rampa no serviço de saúde. Já chamaram 3 vezes mas não tenho como levar porque não tem rampa para entrar; e quando tem rampa não tem corrimão.

Idéia central 2.5: Instalações do serviço de saúde

[DSC 2.5] Não tem lugar para todo mundo esperar, então às vezes esperam no chão e algumas salas não tem como um deficiente entrar.

Idéia central 2.6: Faltam cadeiras de rodas nos serviços de saúde

[DSC 2.6] Não tem cadeira de rodas suficientes no serviço de saúde, é uma disputa por cadeiras ás vezes o deficiente tem que ser carregado no colo.

Idéia central 2.7: Faltam sanitários adequados para as pessoas com deficiências

[DSC 2.7] Nunca vi banheiros para pessoas com deficiência no serviço de saúde, quando tem está muito sujo ou inutilizado (entupido).

Idéia central 2.8: Faltam médicos

[DSC 2.8] No posto de saúde que vou só tem enfermeira, está assim esses dias, não tem médicos.

Idéia central 2.9: Faltam elevadores

[DSC 2.9] No serviço de saúde não tem elevador, tem que usar a escada.

Idéia central 2.10: Problemas com sinalização

[DSC 2.10] A sinalização é insuficiente, tenho que ficar pedindo informações.

O tempo de espera foi um dos fatores relatados como obstáculos no uso dos serviços de saúde e é freqüentemente relatado como problema nos atendimentos ambulatorial ${ }^{7}$ e hospitalar. ${ }^{19}$ Esse período de espera pode ser um fator importante para os pacientes com algum tipo de deficiência uma vez que podem ter necessidades especiais de alimentação, higiene ou descanso. A Lei Federal 10.048 de 8 de novembro de 2000, ${ }^{3}$ garante às pessoas com deficiências, aos idosos com idade igual ou superior a 60 
(sessenta) anos, às gestantes, às lactantes e às pessoas acompanhadas por crianças de colo o direito à prioridade nos atendimentos em repartições públicas. Contudo, em um ambiente hospitalar ou de serviço de saúde, essa priorização pela presença de deficiência pode ser questionada por motivos éticos, uma vez que o deficiente pode não ser o paciente mais necessitado de atendimento em saúde em um determinado momento.

Outro fator que se apresentou como obstáculo ao bom uso dos serviços de saúde pelas pessoas com deficiências foram os problemas com o estacionamento. $\mathrm{O}$ decreto 3.298/99 regulamenta a lei $7.893 / 89^{3}$, que consolida normas de proteção para as pessoas com deficiência, dispõe sobre a obrigatoriedade da presença de vagas reservadas para veículos de pessoas com deficiência ou que transportem essas pessoas em construções de uso coletivo. Esse decreto garante, portanto, vagas reservadas e específicas para os indivíduos com algum tipo de deficiência também nos serviços de saúde, que são de uso coletivo. Entretanto, Mendonça et al $^{15}$ (2007) relatam que a presença de facilidades como estacionamento e localização do serviço pode não ter tanta influência na satisfação com o atendimento.

Aproximadamente um décimo dos entrevistados citaram a ausência de rampas como fator que dificultava o uso dos serviços de saúde. O mesmo decreto 3.298/99 obriga a instalação de rampas ou dispositivos eletromecânicos de deslocamento vertical para a transposição de desníveis entre cômodos dos estabelecimentos. Percebe-se, portanto, que essa dificuldade não deveria ser relatada pelos entrevistados. Também foi citada inexistência de elevadores e de sinalização para as pessoas com deficiências, que são itens previstos na lei 3.298/99.

Algumas inadequações nos serviços foram citadas pelos entrevistados, como local de espera com lugares insuficientes e salas em que as pessoas com deficiência não tinham acesso por obstáculos físicos. Um dos relatos mostra que o direito de ir e vir, previsto em lei pela Constituição, foi ferido no caso de estabelecimentos de saúde cujos obstáculos impossibilitavam a entrada de pessoas com deficiência em suas dependências. 
Um discurso mostrou a necessidade de mais cadeiras de rodas para as pessoas com deficiência, pois em alguns momentos teriam que ser carregadas nos braços para serem atendidos. Isso pode ser um evento que dificulta o uso dos serviços de saúde uma vez que nem sempre o acompanhante do paciente tem condições de fazer o deslocamento do deficiente nos braços, ficando assim, dependentes dos funcionários do serviço de saúde.

Ausência ou inadequação de sanitários destinados às pessoas com deficiência foram relatados pelos entrevistados. A lei 10.098/2000³ garante a construção de sanitários adaptados para as pessoas com deficiências em repartições públicas e sua adequada manutenção.

A ausência de médicos no serviço de saúde também foi relatada por entrevistados, os quais referiram que o atendimento era feito por enfermeiras, não havendo, portanto atendimento médico, somente prestação de cuidados em enfermagem.

Verificou-se também, a ocorrência da idéia central "Nunca tive problemas", que reflete uma adequação do serviço de saúde procurado às necessidades de acessibilidade das pessoas com deficiência. Machado e Nogueira (2008) ${ }^{14}$ também relatam ausência de problemas para utilização de serviços em um grupo de usuários de uma clínica de fisioterapia. Essas informações podem sinalizar que nem sempre a pessoa com deficiência encontra problemas de acessibilidade nos serviços de saúde, levando a crer que uma parcela dessas pessoas tem todas as suas necessidades de acessibilidade aos serviços de saúde satisfeitas. 


\section{CONCLUSÕES}

A discussão destes itens transcende a simples abordagem presença/ausência de obstáculos ao uso de serviços de saúde, alcançando um tema mais amplo e bastante importante em saúde pública: a eqüidade em saúde. A eqüidade é um dos pilares do SUS e, conforme Travassos ${ }^{22}$ (1997) é um princípio de justiça social. Esse conceito se traduziria por: "tratar desigualmente os desiguais". ${ }^{3}$ Assim, diferiria da igualdade, entendida como igualdade de oportunidades. Para uma pessoa com deficiência, não é suficiente ter as mesmas oportunidades quando não há condições de aproveitá-las, comparativamente a alguém que não tenha deficiência. Assim o princípio da eqüidade seria uma maneira de beneficiar as pessoas com deficiências para que tenham igualdade nas oportunidades. Carneiro Junior et $\mathrm{al}^{4}$ (2006) caracterizam a eqüidade como "discriminação positiva" uma vez que é direcionada a grupos populacionais desfavorecidos socialmente, com planejamento e políticas de atenção que busquem a eliminação das desigualdades.

Assim, os problemas relatados pelos entrevistados atuariam de forma a pressionar negativamente as pessoas com algum tipo de deficiência no uso dos serviços de saúde. Esse posicionamento é oposto ao princípio de eqüidade do SUS, baseado na idéia de que todos os indivíduos de uma sociedade devem ter oportunidades iguais para desenvolver seu potencial de saúde, sendo tal sistema que se baseia na eqüidade responsável por atuar contra os obstáculos ao reduzir as diferenças evitáveis ou injustas entre os indivíduos. ${ }^{24}$

A relação de iniqüidade (elemento oposto da eqüidade) e deficiência é descrita na literatura ${ }^{16}$ e aponta diversos obstáculos que atuam contra o correto uso dos serviços de saúde, deixando esse grupo populacional em desvantagem na utilização dos serviços de saúde.

Em conclusão, os discursos expressam que pessoas com algum tipo de deficiência fazem uso de diversos tipos de transporte, demorando períodos variáveis e necessitando de companhia em alguns casos. Problemas na acessibilidade dos serviços de saúde foram relatados pelos sujeitos com deficiências, violando o 
princípio da eqüidade, preceito do Sistema Único de Saúde. A eliminação desses obstáculos poderia ter valor significativo para essa população na utilização dos serviços de saúde, proporcionando possibilidades igualitárias, quando comparadas com as pessoas sem deficiências.

Pesquisa financiada pelo Conselho Nacional de Desenvolvimento Científico Tecnológico $\left(\mathrm{CNPq}\right.$, modalidade apoio de pesquisa acadêmica, Processo $\mathrm{n}^{0}$ : 409724/2006-8) e pela Fundação de Amparo à Pesquisa do Estado de São Paulo (FAPESP, bolsa de doutorado outorgada à Castro SS, Processo n ${ }^{\circ}:$ 2006/59655-2).

Artigo baseado na tese de doutorado de SS Castro, apresentada ao Programa de PósGraduação em Saúde Pública da Faculdade de Saúde Pública, Universidade de São Paulo, em 2010. 


\section{REFERÊNCIAS}

1. Araújo SSC, Freire DBL, Padilha DMP, Baldisserotto J. Suporte social, promoção de saúde e saúde bucal na população idosa no Brasil. Interface (Botucatu). 2006;10(19):203-16. DOI: 10.1590/S1414-32832006000100014

2. Caldas CP. Envelhecimento com dependência: responsabilidades e demandas da família. Cad Saude Publica. 2003;19(3):733-81. DOI:10.1590/S0102311X2003000300009

3. Camargos MCS, Perpetuo IHO, Machado CJ. Expectativa de vida com incapacidade funcional em idosos em São Paulo, Brasil. Rev Panam Salud Publica. 2005;17(5-6):379-86. DOI:10.1590/S1020-49892005000500010

4. Carneiro Jr N, Elias PE. Controle público e eqüidade no acesso a hospitais sob gestão pública não estatal. Rev Saude Publica. 2006;40(5):914-20. DOI:10.1590/S0034-89102006000600023

5. Castro SS, Barros MBA, Carandina L, Alves MCGP, Goldbaum M, Cesar CLG. Deficiência visual, auditiva e física: prevalência e fatores associados em estudo de base populacional. Cad Saude Publica. 2008;24(8):1773-82. DOI:10.1590/S0102311X2008000800006

6. Cheer J, Kroll T, Neri MT, Beatty P. Access barriers for persons with disabilities: the consumer's perspective. J Disabil Pol Studies. 2003;13(4):221-31. DOI:10.1177/104420730301300404

7. Franco SC, Campos GWS. Avaliação da qualidade de atendimento ambulatorial em pediatria em um hospital universitário. Cad Saude Publica. 1998;14(1):61-70. DOI:10.1590/S0102-311X1998000100014

8. Frenk J. El concepto y la medición de accesibilidad. SaludPublica Mexico. 1985;27(5): 438-53.

9. Gill TM, Robison JT, Tinetti ME. Difficulty and dependence: two components of the disability continuum among community-living older persons. Ann Intern Med. 1998;128(2):96-101.

10. Harrison T. Health promotion for persons with disabilities: what does the literature reveal? FamCommunity Health. 2006;29(1Supl):S12-9.

11. Kroll T, Jones GC, Kehn M, Neri MT. Barriers and strategies affecting the utilization of primary preventive services for people with physical disabilities: a qualitative inquiry. Health Soc Care Community. 2006;14(4):284-93. 
DOI:10.1111/j.1365-2524.2006.00613.x

12. Lèfevre F, Lèfevre AMC. Depoimentos e discursos: uma análise em pesquisa social. Brasília: Líber Livro; 2005.

13. Lèfevre F, Lèfevre AMC. O sujeito coletivo que fala. Interface (Botucatu). 2006;10(20): 517-24. DOI:10.1590/S1414-32832006000200017

14. Machado NP, Nogueira LT. Avaliação da satisfação dos usuários de serviços de Fisioterapia. Rev Bras Fisioter. 2008;12(5):401-8. DOI:10.1590/S141335552008000500010

15. Mendonça KMPP, Guerra RO. Desenvolvimento e validação de um instrumento de medida da satisfação do paciente com a fisioterapia. Rev Bras Fisioter. 2007;11(5):369-76. DOI:10.1590/S1413-35552007000500007

16. Merrel J, Cert T, Kinsella F, Murphy F, Philpin S, Ali A. Accessibility and equity of health and social care services: exploring the views and experiences of Bangladeshi carers in South Wales, UK. Health Soc Care Community. 2006;14(3):197-205. DOI:10.1111/j.1365-2524.2006.00610.x

17. Nagai R, Lèfevre AMC, Lèfevre F, Steluti J, Teixeira LR, Zinn LCS, et al. Conhecimentos e práticas de adolescentes na prevenção de acidentes de trabalho: estudo qualitativo. Rev Saude Publica. 2007;41(3):404-11. DOI:10.1590/S003489102007000300012

18. Nosek MA, Hughes RB, Petersen NJ, Taylor HB, Robinson-Whelen S, Byrne M, et al. Secondary conditions in a community-based sample of women with physical disabilities over a 1-year period. Arch Phys Med Rehabil. 2006;87(3):320-7.

DOI:10.1016/j.apmr.2005.11.003

19. Oliveira DF, Arieta CEL, Temporini ER, Kara-José N. Quality of health care: patient satisfaction in a university hospital. Arq Bras Oftalmol. 2006;69(5):731-6. DOI:10.1590/S0004-27492006000500021

20. Othero MB, Dalmaso ASW. Pessoas com deficiência na atenção primária: discurso e prática de profissionais em um centro de saúde-escola. Interface (Botucatu). 2009;13(28):177-88. DOI:10.1590/S1414-32832009000100015

21. Quines S, Cameron I. The use of focus groups with the disabled elderly. Qual Health Res. 1985;5(4):454-62. DOI:10.1177/104973239500500406

22. Travassos C. Eqüidade e o Sistema Único de Saúde: uma contribuição para debate. Cad Saude Publica. 1997;13(2):325-30. DOI:10.1590/S0102311X1997000200024

23. Travassos C, Martins M. Uma revisão sobre os conceitos de acesso e utilização 
de serviços de saúde. Cad Saude Publica. 2004;20(Supl 2):S190-8. DOI: 10.1590/S0102-311X2004000800014

24. Viana ALA, Fausto MCR, Lima LD. Política de saúde e eqüidade. Sao Paulo Perspec. 2003;17(1):58-68. DOI:10.1590/S0102-88392003000100007.

25. Wilson K, Rosenberg MW. Accessibility and the Canadian health care system: squaring perceptions and realities. Health Policy. 2004;67(2):137-48.

DOI:10.1016/S0168-8510(03)00101-5 


\subsection{MANUSCRITO 2}

Artigo submetido ao periódico: Disability and Rehabilitation.

Full title: Problems with accessibility to health services by persons with disabilities in São Paulo

\section{Accessibility to health services in São Paulo}

Shamyr S Castro ${ }^{1,2}$

Alarcos Cieza $^{2}$

Chester LG Cesar ${ }^{1}$

${ }^{1}$ Epidemiology Department - Faculty of Public Health - University of Sao Paulo Brazil

2 Institute for Health and Rehabilitation Sciences - Research Unit for Biopsychosocial Health - ICF Research Branch of WHO CC FIC (DIMDI) Ludwig-Maximilian-University -Munich.

Correspondence and reprints to:

Chester L G Cesar

Epidemiology Department

Faculty of Public Health

Av Dr Arnaldo 715

Cerqueira Cesar - 01246-904 - Sao Paulo - SP - Brasil

Financial support:

- National Council for Scientific and Technological Development (CNPq): proc. $409724 / 2006-8$.

- State of São Paulo Research Foundation (FAPESP): proc. 2006/59655-2.

- German Academic Exchange Service (DAAD) and National Council for Scientific and Technological Development (CNPq): proc. 290006/2008-2. 


\begin{abstract}
Objectives. To describe the occurrence of self-reported problems of accessibility in health services used by persons with disabilities in terms of social and health services variables. Methods. We performed a cross-sectional study designed to assess problems with accessibility in health services faced by persons with disabilities. 333 participants were interviewed at home in São Paulo city, in 2007. Variables about the presence of problem of accessibility, disabilities, gender, age, family head income, ethnicity, use of health services, and others were studied according to frequencies, percentages, $\chi 2$-test, $\chi 2$-test for trend, and Poisson regression models with a significance level of $5 \%$. Results. $15.92 \%$ of the interviewed persons reported problems with accessibility in health services. Persons with multiple disabilities $(\mathrm{PR}=2.91)$ or with a mobility $(\mathrm{PR}=6.46)$ disability had more problems with accessibility than persons with hearing disability. Persons younger than 78 years old had more problems with accessibility as well as those people who needed help to go to the health service $(\mathrm{PR}=3.01)$. Conclusions. Persons with multiple or mobility disability, younger than 78 years and dependent on the assistance of others to go to the health service were more likely to have problems with accessibility in health services. This information can be the first step to the management and/or planning appropriate health services for persons with disabilities.
\end{abstract}

Key words: Persons with Disabilities; Health Services Accessibility; CrossSectional Studies. 


\section{Introduction}

Persons with disabilities are more prone to diseases (CASTRO et al., 2008; CASTRO et al., 2009; NOSEK et al., 2006) and, require health care services more frequently (SIQUEIRA et al., 2009). Paradoxically, people with disabilities represent the population group, who face most obstacles when using these services, including problems of accessibility (CLARKE et al., 2008; GRAY et al., 2003; KROLL et al., 2006; SCHOPP et al., 2002). One recent study, performed in 41 Brazilian cities with populations greater than 10,000 , estimated that $60 \%$ of primary health care services presented problems of architectural accessibility for persons with disability (SIQUEIRA et al., 2009).

Health services are accessible when their use is unrestricted by barriers such as geography, cost, language, and the times when facilities are open (KIGULI et al., 2009). Accessibility is the key to the appropriate use of health services (NOVAES, 2004). It is a feature of the context, and is, in the language of the model of functioning and disability in World Health Organization's (WHO) International Classification of Functioning, Disability and Health (ICF)(WHO, 2001), an environmental factor. In the ICF model, extent of social functioning, including the use of health services, is an outcome of interactions between health conditions and contextual factors, both environmental and personal factors (CLARKE e NIEUWENHUIJSEN, 2009; HOLPER et al., 2009). Person-environment interactions are often very complex as features of the environment may be either facilitators or barriers to participation (SCHNEIDERT et al., 2003).

Knowing when, for whom, and the degree to which the environment is a barrier to the use of health care service is valuable information for policymakers concerned to improve the use of health services by persons with disabilities. Moreover, the investigation of problems of accessibility to health services by persons with disabilities should be a priority for scientists not only in the field of disability research but also in the field of health sciences in general (TOMLINSON et al., 2009).

The objective of this paper is to study the relationship between sociodemographic characteristics and dependency and the occurrence of self-reported problems of accessibility in health services, by persons with disabilities. 


\section{Methods}

\section{Study Design}

The AceSS Study (Accessibility to the Health Services by Persons with Disabilities) was a cross-sectional health survey specially targeting the problems of accessibility to health services by persons with disabilities. It was conducted in São Paulo, 2007.

\section{Study Sample}

The sample was based on two other cross-sectional health surveys carried out in the same city at the years of 2001 and $2003^{\mathrm{O}}$ with persons in the general population. Six hundred sixty nine persons with disabilities who participated in those surveys were selected. The Figure 1 shows the procedure to obtain the final sample.

The inclusion criterion was self-reported disability. The types of disabilities studied were visual, hearing and mobility. Visual disabilities included low vision, partial and total blindness; hearing disabilities included low, partial and total hearing and, mobility disabilities included paralysis and amputation of members or part of them.

\section{Data Collection}

Personal household interviews were performed to collect the data. The instrument used was a semi-structured questionnaire. The questionnaire included a question about the type of disability (visual, hearing, mobility or multiple disabilities), socio-demographic questions (gender, ethnicity, age, family head's income, kind of health insurance (private/public) use of health service and coverage by family health program), two questions about need for help (need for help in daily activities and need for help to go to the health service) and questions about accessibility problems, which were different for the different types of disability. All questions about accessibility problems were summarized in a single dichotomous question, which was used as dependent variable. Problems with accessibility were coded as "yes" if at least one problem with accessibility was reported.

\footnotetext{
${ }^{\circ}$ Cesar CLG, Segri NJ, Sportello R. Inquéritos de saúde no estado de São Paulo [internet]. São Paulo: Faculdade de Saúde Pública da USP; 2005 dez 9 [atualizado 2009 out 13; citado 2010 mai 12]. Available http://hygeia.fsp.usp.br/isa-spwww.fsp.usp.br/isa-sp
} 
In addition, concepts about aspects of the environment and body, which have been showed in ICF, were incorporated in the creation of the questionnaire.

This study was approved by the Ethical Committee from Faculty of Public Health and all participants signed a consent form.

\section{Statistical Analysis}

Descriptive statistics were used to report the problems with accessibility according to the corresponding socio-demographic characteristics.

Differences among the subgroups having/not having problems with accessibility to health services for each of the demographic variables were calculated based on $\chi^{2}$-test; $\chi 2$-test for trend was used for categorical variables and when the assumption of linear trend in the relationship between them and the variable referring to problems with accessibility to the health services applied.

The relation between having problems in accessibility and socio-demographic variables were studied using a Poisson regression with robust variance, as for variables with low frequencies this method is a better option than the logistic regression (BARROS e HIRAKATA, 2003). Prevalence Rates (PR) among persons who had problems with accessibility and persons who did not have, and Confidence Intervals (CI95\%) were calculated with the Poisson regression model.

Having/not having problems in accessibility to health services was the dependent variable and the independent variables were the socio-demographic factors and the two questions about need for help. Categorical variables (type of disability, age and family head's income) were analyzed as "dummies variables" in the regression model. Afterwards, a bivariate Poisson regression model was fitted with the dependent variable and each of the independent variable separately. Lastly, the full Poisson regression model was fitted using the stepwise backward selection and including the variables showing a significant association to the dependent variable in the previous step. We started from a complex model to one more simple, removing the variables according to their significance. In the final model the variables about gender, family head's income and necessity of help in daily $\operatorname{activities}^{\mathrm{P}}$ were identified as confounding variables because when they were in the

\footnotetext{
${ }^{\mathrm{P}}$ Help to bath and dress, to eat, to rise up and/or to walk.
} 
model, they changed the PR of others variables, but they did have statistical significance between $5 \%$ and $20 \%$. Then, the confounding variables were kept in the final model only for adjustment. The statistical significance adopted was 5\% and Stata $9.2 \AA$ was the statistical software used.

\section{Results}

The description of the sample according to the studied variables is presented in the Table 1. From the 669 persons with disability from the 2001 and 2003 study, a final sample of 498 persons was located. From those 333 were interviewed. This information shows a response rate of $67 \%$ about 7 years after the first contact. Among the interviewed persons, 53 (15.92\%) reported problems in accessibility to health services. The persons with mobility disability had accessibility problems (53.85\%) most frequently and those with hearing disabilities least frequently $\left(8.33 \%{ }^{\mathrm{Q}}\right) ; 16.05 \%$ of the men and $15.79 \%$ of the women had accessibility problems; this percentage was $19.75 \%$ among persons with age between 70 and 77 years old and $10.13 \%$ for persons between 78 and 97 years; $22.73 \%$ of the persons with a family head's income eight times larger than the monthly minimum wage (mmw) and $6.52 \%$ of persons with family head's income between 2 and $4.99 \mathrm{mmw}$ had problems with accessibility to health services; $12.34 \%$ of the persons who used the health services in the last year had problems in accessibility; $29.73 \%$ of those who needed help in daily activities had difficulty in accessibility; and $49.06 \%$ of persons who needed help to go to the health services reported accessibility difficulties.

Table 2 shows the non-adjusted and the adjusted PR according to the studied variables. The un-adjusted PRs are significant for mobility $(P R=6.46)$, multiple $(\mathrm{PR}=2.91)$ disability, need for help in daily activities $(\mathrm{PR}=2.09)$ and need for help to go to health services because of the disability $(\mathrm{PR}=3.62)$. The adjusted analysis showed that the persons with multiple $(\mathrm{PR}=2.83)$ or mobility $(\mathrm{PR}=3.08)$ disabilities had more difficulties in accessibility than those with hearing disabilities. Persons between 14 and 54 years old $(P R=2.29)$, between 55 and $69(P R=2.40)$ and between 70 and 97 years old ${ }^{R}(P R=2.23)$ reported more problems in accessibility than those

\footnotetext{
Q 7 persons had low hearing and 1 unilateral deafness

${ }^{\mathrm{R}} 3$ persons were men and 5 were women; 5 had family's head income less than $1 \mathrm{mmw}, 2$ persons with $1 \mathrm{mmw}$, and 1 with family's head income greater than 7,9 mmw.
} 
between 78 and 97 years old. Persons that needed help to go to the health services had more problem in accessibility $(\mathrm{PR}=3.01)$ than those who did not.

\section{Discussion}

The results show that about $16 \%$ of persons with disabilities interviewed had problems with accessibility to health services. Although access or accessibility to health services has already being researched in Brazil (FREIRE et al., 2009; ROCHA e GOES, 2008; RODRIGUES et al., 2009; SCATENA et al., 2009; VIEIRA-DASILVA e ALMEIDA-FILHO, 2009), no study has looked the issue from the point of view of persons with disabilities. HARRINGTON et al., 2009, showed that $2.67 \%$ of 344 persons with physical or intellectual disabilities reported problems with physical access at their physician's office. In the other, $22 \%$ of those with physical or intellectual interviewed reported problems because of the physical layout or location of the health care provider's property, but in this study 8,167 persons were interviewed (CDC, 2006).

Accessibility is an important feature of the overall process of seeking and obtaining health care services and where accessibility is a problem, the result will be a limited use of these services (TRAVASSOS e MARTINS, 2004), this concept can be applied to the persons with disabilities too.

The data indicates that persons with mobility or multiple disabilities had more accessibility problems than those with hearing disability (LAWTHERS et al., 2003), likely because the physical environment poses more problems for them. Physical obstacles can be divided into structural-environmental barriers (facility, equipment, and transportation) and process barriers (appointment scheduling, patient-provider communication, professional manner, disability-specific knowledge, information and self-education) (KROLL et al., 2006). When accessibility is not adequate, it hinders the use of health service. Although the impact of the environment on persons with disabilities was studied before the ICF came on the scene (LIVNEH, 1987; MASALA e PETRETO, 2008), the ICF helps to explain the role of the environment in the disabling process. As a result, the ICF can serve as a tool for the more easily identifying barriers and facilitators related to the environment (RIMMER, 2006).

The results show that the persons with disabilities younger than 78 years old referred more problems in accessibility than those between 78 and 97 years old. The 
relationship between age and disability has been addressed in several studies (BERLAU et al., 2003; CLARKE e NIEUWENHUIJSEN, 2009), as has the relationship of reduction of functionality and the aging process (FRISARD et al., 2007). However, the data found in this study suggests the opposite, that younger people experience more accessibility problems. The higher rate of home medical visits among older people (BENTO e BROFMAN, 2009; CHAN et al., 2009; KELLY e ORR, 2009; LAPLANT et al., 2002; OHWAKI et al., 2009; RODRIGUES et al., 2009) or the low frequency of occurrence ( 8 persons) may explain this fact.

Research on the problems with accessibility to health services is uncommon. Such studies would be useful to health managers, a fact recognized by the Ministry of Health of Brazil and the National Council for Scientific and Technological Development $(\mathrm{CNPq})$, who help to fund the present research and others.

This study has the limitation that disabilities were self-reported without clinical confirmation. A more detailed and standardized measurement of functionality would probably better delineate the true source of the accessibility problem confronted. Second, each disability should be studied separately. Third, an adjust by time of disability should be made. Fourth, the disabilities should be graduated in order to separate the most incapacitated. Still, this study is a first step toward better management and planning of health services for persons with disabilities. Additional studies are needed to increase our knowledge about the influence of the environmental factors on accessibility to health services. 


\section{Bibliography}

Barros AJ, Hirakata VN. Alternatives for logistic regression in cross-sectional studies: an empirical comparison of models that directly estimate the prevalence ratio. BMC Med Res Methodol, 2003; 3:21.

Bento VFR, Brofman PRS. Impacto da consulta de enfermagem na frequência de internações em pacientes com insuficiência cardíaca em Curitiba - Paraná. Arq Bras Cardiol, 2009; 92(6): 490-496.

Berlau DJ, Corrada MM, Kawas C. The prevalence of disability in the oldest-old is high and continues to increase with age: findings from The 90+ Study. Int J Geriatr Psychiatry, 2009; 24(11):1217-25.

Castro SS, César CLG, Carandina L, Barros MBA, Alves MCGP, Goldbaum M. Deficiência visual, auditiva e física: prevalência e fatores associados em estudo de base populacional. Cad. Saúde Publica, 2008; 24(8): 1773-1782.

Castro SS, César CLG, Carandina L, Barros MBA, Alves MCGP, Goldbaum M. Physical Disability, Recent Illnesses and Health Self-Assessment in a PopulationBased Study in São Paulo, Brazil. Disabil Rehabil, 2009 (in press)

CDC - Centers for Disease Control and Prevention. Environmental barriers to health care among persons with disabilities--Los Angeles County, California, 2002-2003. MMWR Morb Mortal Wkly Rep.,, 2006; 55(48):1300-3.

Chan L, Wang H, Terdiman J, Hoffman J, Ciol MA, Lattimore BF, Sidney S, Quesenberry C, Lu Q, Sandel ME. Disparities in outpatient and home health service utilization following stroke: results of a 9-year cohort study in Northern California. PM R., 2009;1(11): 997-1003.

Clarke P, Nieuwenhuijsen ER. Environments for healthy ageing: a critical review. Maturitas, 2009; 64: 14-9.

Clarke P, Ailshire JA, Bader M, Morenoff JD, House JS. Mobility disability and the urban built environment. Am J Epidemiol., 2008; 168(5):506-13.

Freire DB, Gigante LP, Béria JU, Palazzo Ldos S, Figueiredo AC, Raymann BC. Access by hearing-disabled individuals to health services in a southern Brazilian city. Cad Saude Publica., 2009; 25(4): 889-97.

Frisard MI, Fabre JM, Russell RD, King CM, DeLany JP, Wood RH, Ravussin E. Physical activity level and physical functionality in nonagenarians compared to individuals aged 60-74 years. J Gerontol A Biol Sci Med Sci., 2007; 62(7): 783-788.

Gray DB, Gould M, Bickenbach JE. Environmental barriers and disability. J Arch Plan Res., 2003; 20(1): 29-37.

Harrington AL, Hirsch MA, Hammond FM, Norton HJ, Bockenek WL. Assessment of Primary Care Services and Perceived Barriers to Care in Persons with Disabilities. Am J Phys Med Rehabil., 2009; 88(10): 852-863.

Holper L, Coenen M, Weise A, Stucki G, Cieza A, Kesselring J. Characterization of functioning in multiple sclerosis using the ICF. J Neurol., 2009; 257(1):10313.

Iezzone LI, McCarthy EP, Davis RB, Siebens H. Mobility impairments and use of screening and preventive services. Am J Public Health., 2000; 90: 955-961. 
Kelly J, Orr A. Accountability, responsiveness and quality for clients model of home support: a model for improved home support services to promote aging at home. Healthc Pap., 2009; 10(1): 65-71.

Kiguli J, Ekirapa-Kiracho E, Okui O, Mutebi A, MacGregor H, Pariyo G. Increasing access to quality health care for the poor: community perceptions on quality care in Uganda. Patient Prefer Adherence, 2009: 3: 77-85.

Kroll T, Jones GC, Kehn M, Neri MT. Barriers and strategies affecting the utilisation of primary preventive services for people with physical disabilities: a qualitative inquiry. Health Soc Care Community, 2006; 14(4): 284-93.

LaPlante MP, Harrington C, Kang T. Estimating paid and unpaid hours of personal assistance services in activities of daily living provided to adults living at home. Health Serv Res., 2002; 37(2): 397-415.

Lawthers AG, Pransky GS, Peterson LE, Himmelstein JH. Rethinking quality in the context of persons with disability. Int J Qual Health Care, 2003; 15(4): 28999.

Livneh H. Person-environment congruence: a rehabilitation perspective. Int $\mathbf{J}$ Rehabil Res, 1987; 10(1): 3-19.

Masala C, Petretto DR. From disablement to enablement: conceptual models of disability in the $20^{\text {th }}$ century. Disabil Rehabil., 2008; 30(17): 1233-1244.

Nosek MA, Hughes RB, Petersen NJ, Taylor HB, Robinson-Whelen S, Byrne M, Morgan R. Secondary conditions in a community-based sample of women with physical disabilities over a 1-year period. Arch Phys Med Rehabil, 2006; 87(3): 320-7

Novaes, Hillegonda Maria Dutilh. Pesquisa em, sobre e para os serviços de saúde: panorama internacional e questões para a pesquisa em saúde no Brasil. Cad Saude Publica, 2004; 20(suppl.2): S147-S157.

Ohwaki K, Hashimoto H, Sato M, Tamiya N, Yano E. Predictors of continuity in home care for the elderly under public long-term care insurance in Japan. Aging Clin Exp Res., 2009; 21(4-5): 323-8.

Rimmer JH. Use of the ICF in identifying factors that impact participation in physical activity/rehabilitation among people with disabilities. Disabil Rehabil., 2006; 28(17): 1087-1095.

Rocha RA, Goes PS. Comparison of access to Oral Health Services between areas covered and not covered by the Family Health Program in Campina Grande, Paraíba State, Brazil. Cad Saude Publica., 2008; 24(12): 2871-80.

Rodrigues MA, Facchini LA, Piccini RX, Tomasi E, Thumé E, Silveira DS, Siqueira FV, Paniz VM. Use of primary care services by elderly people with chronic conditions, Brazil. Rev Saude Publica., 2009; 43(4): 604-12.

Scatena LM, Villa TC, Netto AR, Kritski AL, Figueiredo TM, Vendramini SH, Assis MM, Motta MC. Difficulties in the accessibility to health services for tuberculosis diagnoses in Brazilian municipalities. Rev Saude Publica. 2009; 43(3): 389-97.

Schneidert M, Hurst R, Miller J, Üstün B. The role of environment in the International Classification of Functioning, Disability and Health (ICF). Disabil Rehabil, 2003; 25(11-12): 588-595. 
Schopp LH, Sanford TC, Hagglund KJ, Gay JW, Coatney MA. Removing service barriers for women with physical disabilities: promoting accessibility in the gynecologic care setting. J Midwifery Womens Health, 2002; 47(2): 74-9.

Siqueira FCV, Facchini LA, Silveira DS, Piccini RX, Thumé E, Tomasi E. Architectonic barriers for elderly and physically disabled people: an epidemiological study of the physical structure of health service units in seven Brazilian states. Cien Saude Colet., 2009; 14(1): 39-44.

Travassos C, Martins M. A review of concepts in health services access and utilization. Cad Saude Publica., 2004; 20(2): S190-S198.

Tomlinson M, Swartz L. Officer A, Chan KY, Rudan I, Saxena S. Research priorities for health of people with disabilities: an expert opinion exercise. Lancet, 2009; 374: 1857-62.

Vieira-da-Silva LM, Almeida Filho N. Health equity: a critical analysis of concepts. Cad Saude Publica., 2009; 25(Suppl 2): S217-26.

World Health Organisation. International Classification of Functioning, Disability and Health. Geneva, Switzerland: World Health Organization; 2001. 
Figure 1: Description of the process of obtaining the final sample and interviews, AceSS, Sao Paulo, 2007.

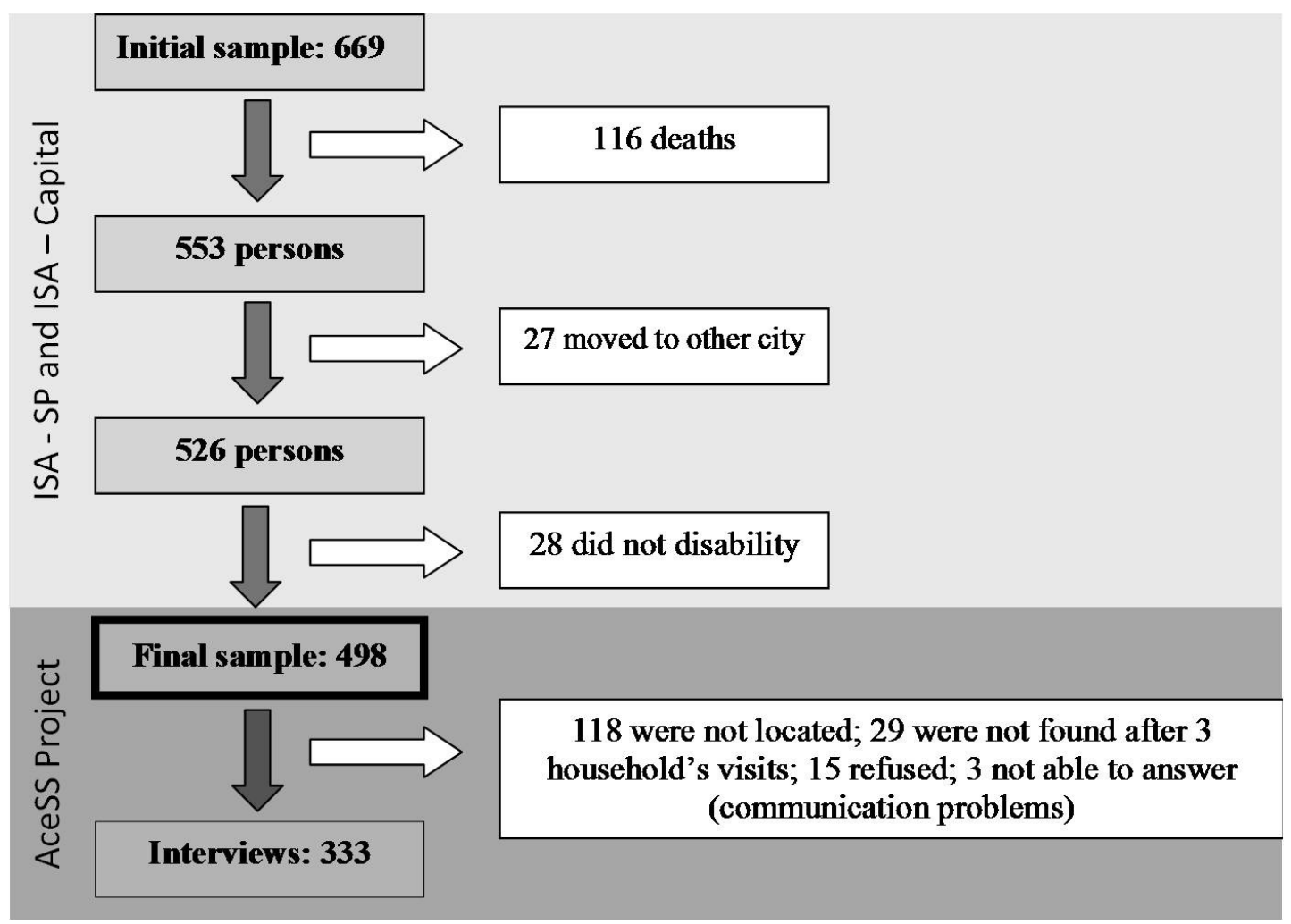


Table 1: Demographic characteristics of participants and studied variables according to problems with accessibility to the health services, AceSS, Sao Paulo, 2007.

\begin{tabular}{|c|c|c|c|c|c|c|c|}
\hline \multirow{3}{*}{ Variables } & \multicolumn{6}{|c|}{ Problems with accessibility to health services } & \multirow{3}{*}{$\mathbf{p}$} \\
\hline & \multicolumn{2}{|c|}{ No } & \multicolumn{2}{|c|}{ Yes } & \multicolumn{2}{|c|}{ Total } & \\
\hline & $\mathbf{n}(\mathbf{2 8 0})$ & $\%(84.08)$ & $\mathbf{n}(\mathbf{5 3})$ & $\%(15.92)$ & $\mathbf{n}(\mathbf{3 3 3})$ & $\%(100.00)$ & \\
\hline Disability & & & & & & & $<0.01^{(1)}$ \\
\hline Visual & 124 & 90.51 & 113 & 9.49 & 137 & 100.00 & \\
\hline Hearing & 88 & 91.67 & 8 & 8.33 & 96 & 100.00 & \\
\hline Mobility & 12 & 46.15 & 14 & 53.85 & 26 & 100.00 & \\
\hline Multiple & 56 & 75.68 & 18 & 24.32 & 74 & 100.00 & \\
\hline Gender & & & & & & & $>0.05^{(1)}$ \\
\hline M & 136 & 83.95 & 26 & 16.05 & 162 & 100.00 & \\
\hline $\mathbf{F}$ & 144 & 84.21 & 27 & 15.79 & 171 & 100.00 & \\
\hline Age (years) & & & & & & & $<0.05^{(2)}$ \\
\hline $14-54$ & 73 & 84.88 & 13 & 15.12 & 86 & 100.00 & \\
\hline $55-69$ & 71 & 81.61 & 16 & 18.39 & 87 & 100.00 & \\
\hline 70-77 & 65 & 80.25 & 16 & 19.75 & 81 & 100.00 & \\
\hline 78-97 & 71 & 89.87 & 8 & 10.13 & 79 & 100.00 & \\
\hline Family head's income ${ }^{(3)}$ & & & & & & & $<0.05^{(2)}$ \\
\hline$<1$ & 170 & 82.93 & 35 & 17.07 & 205 & 100.00 & \\
\hline 1 & 31 & 79.49 & 8 & 20.51 & 39 & 100.00 & \\
\hline $2-4.99$ & 43 & 93.48 & 3 & 6.52 & 46 & 100.00 & \\
\hline $5-7.99$ & 19 & 90.48 & 2 & 9.52 & 21 & 100.00 & \\
\hline$>8$ & 17 & 77.27 & 5 & 22.73 & 22 & 100.00 & \\
\hline Ethnicity & & & & & & & $>0.05^{(1)}$ \\
\hline Caucasian & 167 & 84.77 & 30 & 15.23 & 197 & 100.00 & \\
\hline Black/Mulattoes & 106 & 83.46 & 21 & 16.54 & 127 & 100.00 & \\
\hline Others & 7 & 77.78 & 2 & 22.22 & 9 & 100.00 & \\
\hline Use of health service ${ }^{(4)}$ & & & & & & & $<0.01^{(1)}$ \\
\hline No & 74 & 75.51 & 24 & 24.49 & 98 & 100.00 & \\
\hline Yes & 206 & 87.66 & 29 & 12.34 & 235 & 100.00 & \\
\hline Necessity of help in daily activities ${ }^{(5)}$ & & & & & & & $<0.02^{(1)}$ \\
\hline No & 254 & 85.81 & 42 & 14.19 & 296 & 100.00 & \\
\hline Yes & 26 & 70.27 & 11 & 29.73 & 37 & 100.00 & \\
\hline Private Health Insurance & & & & & & & $>0.05^{(1)}$ \\
\hline No & 184 & 82.88 & 38 & 17.12 & 222 & 100.00 & \\
\hline Yes & 92 & 85.98 & 15 & 14.02 & 107 & 100.00 & \\
\hline Coverage by Family Health Program & & & & & & & $>0.05^{(1)}$ \\
\hline No & 256 & 84.77 & 46 & 15.23 & 302 & 100.00 & \\
\hline Yes & 24 & 77.42 & 7 & 22.58 & 31 & 100.00 & \\
\hline $\begin{array}{l}\text { Necessity to help to go to health service } \\
\text { because the disability }\end{array}$ & & & & & & & $<0.01^{(1)}$ \\
\hline No & 233 & 84.42 & 43 & 15.58 & 276 & 100.00 & \\
\hline Yes & 27 & 50.94 & 26 & 49.06 & 53 & 100.00 & \\
\hline Notes: & & & & & & & \\
\hline (1) $\chi 2$-test & & & & & & & \\
\hline (2) Linear tendency test & & & & & & & \\
\hline (3) Monthly minimum wage & & & & & & & \\
\hline${ }^{(4)}$ In the last year & & & & & & & \\
\hline $\begin{array}{l}\text { (4) Help to bath and dress, to eat, to rise } \\
\text { (6) The last health service used was consi }\end{array}$ & walk. & & & & & & \\
\hline
\end{tabular}


Table 2: Prevalence Ratio (PR) from bivariate analysis and final Poisson regression model of the occurrence of problems with accessibility to health services by persons with disabilities according to socio-demographic characteristics AceSS, Sao Paulo, 2007.

\begin{tabular}{|c|c|c|c|c|c|c|}
\hline Variables & $\mathbf{P R}^{(1)}$ & CI $(95 \%)$ & p & $\overline{P \mathbf{R}^{(2)}}$ & CI $(95 \%)$ & $\mathbf{p}$ \\
\hline \multicolumn{7}{|l|}{ Disability } \\
\hline Visual & 1.13 & $0.49-2.64$ & $>0.05$ & 1.11 & $0.48-2.57$ & $>0.05$ \\
\hline Hearing & 1.00 & & & 1.00 & & \\
\hline Mobility & 6.46 & $3.04-13.7$ & $<0.01$ & 3.08 & $1.25-7.59$ & $<0.02$ \\
\hline Multiple & 2.91 & $1.34-6.34$ & $<0.01$ & 2.83 & $1.26-6.33$ & $<0.02$ \\
\hline \multicolumn{7}{|l|}{ Gender } \\
\hline M & 1.00 & & & & & \\
\hline $\mathbf{F}$ & 0.98 & $0.59-1.61$ & $>0.05$ & 1.03 & $0.63-1.68$ & (a) \\
\hline \multicolumn{7}{|l|}{ Age (years) } \\
\hline $14-54$ & 1.66 & $0.29-9.47$ & $>0.05$ & 2.29 & $1.02-5.15$ & $<0.05$ \\
\hline $55-69$ & 1.28 & $0.20-8.18$ & $>0.05$ & 2.40 & $1.12-5.14$ & $<0.03$ \\
\hline 70-77 & 2.14 & $0.39-11.73$ & $>0.05$ & 2.23 & $1.06-4.68$ & $<0.04$ \\
\hline 78-97 & 1.00 & & & 1.00 & & \\
\hline \multicolumn{7}{|l|}{ Family head's income ${ }^{(3)}$} \\
\hline$<1$ & 2.61 & $0.84-8.15$ & $>0.05$ & 1.83 & $0.68-4.45$ & (a) \\
\hline 1 & 3.14 & $0.89-11.07$ & $>0.05$ & 1.81 & $0.61-5.37$ & (a) \\
\hline $2-4.99$ & 1.00 & & & 1.00 & & \\
\hline $5-7.99$ & 1.46 & $0.26-8.12$ & $>0.05$ & 0.87 & $0.17-4.31$ & (a) \\
\hline$>8$ & 3.48 & $0.91-13.31$ & $>0.05$ & 1.62 & $0.49-5.36$ & (a) \\
\hline \multicolumn{7}{|c|}{ Necessity of help in daily activities ${ }^{(4)}$} \\
\hline No & 1.00 & & & 1.00 & & \\
\hline Yes & 2.09 & $1.18-3.70$ & $<0.02$ & 0.74 & $0.39-1.40$ & (a) \\
\hline \multicolumn{7}{|c|}{$\begin{array}{l}\text { Necessity of help to go to health } \\
\text { service because the disability }\end{array}$} \\
\hline No & 1.00 & & & 1.00 & & \\
\hline Yes & 3.62 & $2.26-5.80$ & $<0.01$ & 3.01 & $1.82-5.07$ & $<0.01$ \\
\hline
\end{tabular}

Notes:
(a) $0.05<\mathrm{p}<0.20$
${ }^{(1)}$ Bivariate analysis with Poisson regression model
(2) Adjusted for all variables from the Poisson regression model.
${ }^{(3)}$ Monthly minimum wage.
(4) Help to bath and dress, to eat, to rise up and/or to walk.
(5) The last health service used was considered. 


\subsection{MANUSCRITO 3}

Artigo não submetido. Sugestão de periódico Revista Brasileira de Fonoaudiologia

Dificuldades na comunicação entre pessoas com deficiência auditiva e profissionais de saúde: uma questão de saúde pública

Shamyr Sulyvan de Castro; CASTRO, SS

Karina M Paiva, Paiva, KM

Chester Luiz Galvão Cesar; CESAR, CLG

Departamento de Epidemiologia - Faculdade de Saúde Pública FSP - USP

Avenida Dr Arnaldo 715 - 01246-904 - São Paulo-SP

\section{Resumo}

Objetivos. Estudar a ocorrência de dificuldades para ouvir e entender profissionais de estabelecimentos de saúde, em um grupo de pessoas com deficiência auditiva e múltipla (auditiva e visual e/ou mobilidade), e discutir o problema no âmbito das políticas públicas de saúde e suas implicações na saúde desses indivíduos. Métodos. Estudo transversal, realizado no ano de 2007, na cidade de São Paulo e região, sobre os problemas de acessibilidade de pessoas com deficiência aos serviços de saúde, por meio de entrevista domiciliar com questionário semiestruturado. Foram entrevistados 160 indivíduos com deficiência auditiva e/ou deficiência múltipla. Resultados. Problemas para ouvir e entender os profissionais de saúde no último serviço visitado foram relatados por 35\% dos entrevistados; 30,6\% (IC: 23,4-37,8) apontaram dificuldades para entender os médicos; 18,1\% (IC: 12,0-24,1), para entender as enfermeiras; e 21,2\% (IC: 14,8-27,6) para entender os outros funcionários. Não houve diferenças estatísticas quando se considerou as variáveis sexo, idade, renda do chefe da família, raça ou etnia, necessidade de ajuda para tomar banho e vestir-se, comer, levantar-se e/ou andar, possuir ou não plano privado de saúde e tipo de serviço de saúde visitado. Discussão. As dificuldades para ouvir e entender os profissionais de saúde foram relatadas por $35 \%$ dos entrevistados e não apresentaram relação direta com as variáveis independentes estudadas. Tais dificuldades podem levar à menor utilização dos serviços de saúde, implicando em prejuízos para a saúde das pessoas com deficiência auditiva e ferindo o princípio da equidade. Conclusão. Esses dados podem fornecer suporte para as políticas públicas vigentes, no sentido de avaliar, planejar e reformular ações em saúde específicas para esse grupo de pessoas, promovendo o uso dessas informações voltadas para a gestão das políticas, para garantir a equidade em saúde. 


\section{Introdução}

O grupo populacional constituído pelas pessoas com algum tipo de deficiência totaliza cerca de 600 milhões no mundo, segundo dados da Organização das Nações Unidas (ONU, 2008). No Brasil, aproximadamente 14,5\% da população referiu algum tipo de deficiência no censo demográfico do ano de 2000 (IBGE, 2003). Entre estes, mais de cinco milhões relataram deficiência auditiva, incluindo incapacidade auditiva e dificuldade de ouvir. Observou-se uma porcentagem de $11,1 \%$ de pessoas com algum tipo de deficiência em áreas do estado de São Paulo (CASTRO et al., 2008).

A deficiência auditiva caracteriza-se pela perda parcial ou total da capacidade de ouvir (BRASIL, 2003), manifestando-se em diferentes graus: leve, moderado, severo e profundo, em virtude da redução da sensibilidade ou da discriminação auditiva. As consequências mais importantes da deficiência auditiva estabelecidas pela OMS estão relacionadas à incapacidade auditiva e à desvantagem auditiva. A primeira relaciona-se ao comprometimento do desempenho e da habilidade do indivíduo para utilizar a audição nas atividades diárias, como percepção dos sons de fala e do ambiente. A outra repercute no desempenho de suas atividades e de seu papel na sociedade, assim como em suas aspirações educacionais, sociais e ocupacionais em decorrência da deficiência e da incapacidade auditiva (MARQUES et al., 2004).

Esse tipo de deficiência prejudica a compreensão da fala, interferindo no processo de comunicação e exigindo das pessoas com deficiência auditiva a utilização de estratégias de comunicação para melhor compreensão da fala. Essas estratégias constituem-se em um conjunto de atitudes que facilitam a compreensão da mensagem, garantindo a efetividade da comunicação (JACOB et al., 2000). O uso da leitura orofacial é uma das estratégias mais utilizadas pelas pessoas com acometimentos auditivos, feita de forma inconsciente ao se comunicar por meio da observação da expressão facial, gestos, mudança de postura e pistas que auxiliam na decodificação da informação. Assim, esses indivíduos tendem a apresentar um perfil de saúde e de uso dos serviços de saúde diferenciado exigindo maior atenção e cuidado no seu atendimento (FREIRE et al., 2009).

As pessoas com deficiência necessitam de cuidados em saúde direcionados, por constituírem um grupo heterogêneo que reúne indivíduos com peculiaridades inerentes à sua deficiência (FREIRE et al., 2009).

O conhecimento dessas questões por parte dos profissionais de saúde é essencial na tentativa de se obter sucesso na comunicação, impedindo que essa deficiência possa afetar a troca de informações entre a pessoa com deficiência auditiva e os profissionais de saúde (BERNARDES et al., 2009; PAGLIUCA et al., 2007).

Considerando o perfil de saúde diferenciado das pessoas com deficiência; as dificuldades no uso dos serviços de saúde; a possibilidade de que problemas possam ser evidenciados e a chance de que, posteriormente, essas informações possam servir de orientação na criação de medidas, ações ou políticas em saúde para a melhoria do atendimento a essas pessoas, estudos que investiguem as dificuldades encontradas pelas pessoas com deficiência devem ser estimulados.

\section{Objetivo}


Estudar a ocorrência de dificuldades para ouvir e entender profissionais de estabelecimentos de saúde (médicos, enfermeiros, recepcionistas, atendentes e outros funcionários), por um grupo de pessoas com deficiência auditiva ou múltipla (auditiva e outra).

\section{Metodologia}

Um estudo transversal foi realizado na cidade de São Paulo no ano de 2007, o estudo de acessibilidade aos serviços de saúde de pessoas com deficiência, AceSS 2007. As informações foram coletadas por entrevistas domiciliares, utilizando um questionário semi-estruturado como instrumento de coleta de dados. O questionário é composto de diferentes blocos, com perguntas específicas para cada tipo de deficiência estudada (mobilidade, visual e/ou auditiva), além de partes que foram respondidas por todas as pessoas, independentemente da deficiência. Utilizaram-se, no presente artigo, as informações sobre problemas para ouvir e entender completamente o que médicos, enfermeiros, atendentes, recepcionistas e outros funcionários disseram durante atendimentos em estabelecimentos de saúde. A equipe de entrevistadores de campo recebeu capacitação específica para a aplicação do questionário e $10 \%$ das entrevistas foram checadas aleatoriamente pelos supervisores de campo.

Os sujeitos do estudo AceSS foram selecionados a partir de dois inquéritos de saúde conduzidos no ano de 2001 (ISA-Capital) e 2003 (ISA-SP) ${ }^{\mathrm{S}}$. As pessoas que referiram alguma deficiência nesses dois inquéritos foram selecionadas, listadas, localizadas e novamente entrevistadas no estudo AceSS 2007. Inicialmente, foram selecionadas 669 pessoas. Uma pesquisa de campo para localização dos sujeitos a serem entrevistados verificou que 116 pessoas haviam falecido, 27 mudaram-se para fora da área estudada e 28 pessoas tiveram as deficiências corrigidas ${ }^{\mathrm{T}}$. Esses indivíduos foram excluídos da pesquisa por não apresentarem as características da amostra pretendida. Outras 118 pessoas não foram localizadas, 15 recusaram-se a participar, 29 não foram encontradas em casa após três visitas em dias e horários diferentes e três não estavam aptas a responder e o proxy não sabia as respostas. Esses casos foram considerados perdas amostrais da pesquisa. Assim, partindo-se de uma amostra inicial de 669 pessoas, 171 foram excluídas porque faleceram ou não faziam mais parte da amostra, restando então, 498 pessoas. Registrou-se uma perda de 165 indivíduos. Dessa forma, 333 pessoas foram entrevistadas no ano de 2007, aproximadamente seis anos após o primeiro contato, obtendo-se uma taxa de resposta de $66,9 \%$.

A variável dependente, sobre os problemas para ouvir e entender o que os funcionários de estabelecimentos de saúde disseram foram coletadas em três perguntas específicas que diziam respeito ao último serviço de saúde utilizado. As pergutnas foram feitas da seguinte forma: Em decorrência de sua dificuldade de audição, o(a) senhor(a) teve algum problema para ouvir e entender o que o médico disse?, aplicando-se também a enfermeiros e outros funcionários ${ }^{\mathrm{U}}$. Posteriormente,

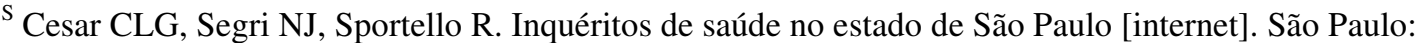
Faculdade de Saúde Pública da USP; 2005 dez 9 [atualizado 2009 out 13]. Disponível em:

$<$ http://hygeia.fsp.usp.br/isa-sp>. Acesso em 10 maio 2010

${ }^{\mathrm{T}}$ Correções por cirurgias e/ou outros tratamentos médicos.

${ }^{\mathrm{U}}$ Recepcionistas, atendentes e outros funcionários de nível médio de ensino.
} 
foi criada uma variável para agregar as respostas das três questões. Além disso, foram estudados, como variáveis independentes, o sexo, a idade (categorizada em quartis: 14-54 anos, 55-69 anos, 70-77 anos, 78-97 anos), a renda do chefe da família em salários mínimos por mês ( $<1 \mathrm{sm}, 1 \mathrm{sm}, 2-4,99 \mathrm{sm}, 5-7,99 \mathrm{sm},>8 \mathrm{sm})$, a raça ou etnia (branco, negro/mulato, outros), a necessidade ou não de ajuda para tomar banho e vestir-se, comer, levantar-se e/ou andar, o fato de o entrevistado possuir ou não plano de saúde privado e o serviço de saúde procurado pela última vez (UBS/AMA/APA/ESF/PSF, consultório médico, ambulatório, emergência, hospital, outros.).

A significância adotada foi de 5\% e testada com teste de Qui-Quadrado. O software EpiData foi usado na digitação dos dados e o Stata 9.2® na sua análise.

A presente pesquisa foi aprovada pelo comitê de ética da Faculdade de Saúde Pública da Universidade de São Paulo, por meio do protocolo número 1653.

\section{Resultados}

Foram estudadas 160 pessoas, que faziam parte da amostra do estudo AceSS 2007, que referiram ter deficiência auditiva (138 com baixa audição e 22 com surdez unilateral) ou deficiência auditiva associada a outra deficiência (visual ou de mobilidade).

As características sociodemográficas da população estudada, segundo problemas para ouvir e entender os profissionais de saúde, assim como as características do atendimento, como o tipo de plano de saúde, o serviço procurado e a necessidade de ajuda estão descritos no Quadro 1.

Entre as pessoas com deficiência estudadas na amostra, 35\% relataram problemas para ouvir e entender o que foi dito por profissionais de saúde no último serviço de saúde utilizado. Desses, 30,6\% (IC: 23,4-37,8) tiveram problemas para ouvir e entender o que os médicos disseram; 18,1\% (IC: 12,0-24,1) o que as enfermeiras disseram; e 21,2\% (IC:14,8-27,6), o que recepcionistas, atendentes e outros funcionários disseram (dados não mostrados em tabela).

Na Tabela 1, observa-se a relação entre os problemas para ouvir e entender o que os profissionais de saúde disseram por ocasião do último serviço de saúde procurado pelos entrevistados com as variáveis sociodemográficas, tipo de atendimento e necessidade de ajuda.

Foram verificadas pequenas diferenças nas variáveis tipo de deficiência e sexo. Entre as pessoas que relataram ter somente deficiência auditiva, 34,7\% tiveram problemas para ouvir e entender o que os profissionais de saúde disseram. Essa porcentagem é de 35,4\% para o grupo de pessoas com deficiência auditiva associada a outro tipo de deficiência. Em relação ao sexo 35,3\% das pessoas do sexo masculino relataram problemas para ouvir e entender o que foi dito por profissionais de saúde, sendo que entre as pessoas do sexo feminino, a porcentagem foi de $34,7 \%$.

A ocorrência dos problemas de comunicação estudados foi relatada por $28,6 \%$ das pessoas com idade entre 14 e 54 anos; $35 \%$ dos entrevistados de 55 a 69 anos; $37,2 \%$ dos sujeitos de 70 a 77 anos; e por $36,7 \%$ dos indivíduos com idade entre 78 e 97 anos.

A distribuição da variável de estudo segundo renda do chefe da família foi de $33,7 \%$ para pessoas de famílias com chefes que ganhavam menos de um salário 
mínimo, de $50 \%$ entre os entrevistados pertencentes a famílias com renda de um salário mínimo; $35 \%$ para a renda entre dois a cinco salários mínimos; $25 \%$ para renda entre cinco e oito salários mínimos; e 27,3\% para pessoas com renda do chefe da família maior que oito salários mínimos.

Considerando-se a necessidade de ajuda para tomar banho e vestir-se, comer, levantar-se e/ou andar, 27,8\% das pessoas que não precisavam de ajuda relataram ter tido problemas para ouvir e entender o que foi dito pelos profissionais dos estabelecimentos de saúde enquanto $37,1 \%$ dos entrevistados que precisavam de ajuda tiveram o mesmo problema. Dos entrevistados que não possuíam plano de saúde privado, 34,5\% tiveram dificuldades para ouvir e entender o que foi dito por profissionais de saúde. Essa porcentagem foi de 38,9\% entre as pessoas que tinham plano de saúde particular.

Os dados dos entrevistados que usaram as unidades básicas de saúde e correlatos $^{\mathrm{V}}$ na última visita a um serviço de saúde mostraram que 30,5\% deles não conseguiram ouvir e entender o que lhes foi dito. Essa porcentagem é de $23,5 \%$ entre os entrevistados que foram ao consultório médico na última vez em que usaram um serviço de saúde; de $50 \%$ para aqueles que foram a um ambulatório; $50 \%$ para os que procuraram um serviço de emergência; $43,2 \%$ para os que foram diretamente ao hospital; e de $20 \%$ para os entrevistados que utilizaram outros serviços.

Não foi observada associação estatisticamente significante entre os problemas para ouvir e entender o que foi dito pelos profissionais de saúde no último serviço de saúde visitado e as variáveis sociodemográficas, tipo de atendimento e necessidade de ajuda.

\section{Discussão}

Entre as pessoas que relataram algum déficit auditivo, 35\% confirmaram dificuldades para ouvir e entender o que os profissionais de saúde disseram na última visita a algum serviço de saúde. Esses relatos sugerem um despreparo dos profissionais de saúde e do próprio serviço para atender a essa demanda populacional, no que se refere a recursos alternativos e maior disponibilidade de tempo na tentativa de uma comunicação eficiente, essencial para o sucesso do atendimento.

A maior porcentagem relatada de não entendimento dos profissionais de saúde foi atribuída aos médicos $(30,6 \%)$, o que pode ser apontado como consequência de diversos fatores, como o curto tempo de realização das consultas nos serviços de saúde, decorrente da grande demanda de atendimento, e o despreparo para o atendimento por parte dos profissionais de saúde, considerando também que o médico pode ser o profissional de saúde com quem as pessoas têm mais contato. Além disso, CARDOSO et al. relatam que as dificuldades de comunicação nos serviços de saúdes para o grupo de pessoas com deficiência auditiva severa que estudaram, não se restringia somente aos médicos, mas a outros profissionais da área como farmacêutico e dentista e também à funcionários como secretárias e recepcionistas, informação corroborada por outros estudos (IEZZONI et al., 2004; McALEER, 2006; PEREIRA e FORTES, 2010).

\footnotetext{
${ }^{\mathrm{v}} \mathrm{UBS} / \mathrm{AMA} / \mathrm{APA} / \mathrm{ESF} / \mathrm{PSF}$
} 
Não foram verificadas diferenças estatisticamente significantes entre a dificuldade de ouvir e entender o que foi dito pelos profissionais de saúde e o tipo de deficiência; sexo; idade; renda do chefe da família; raça ou etnia; necessidade de ajuda para tomar banho e vestir-se, comer, levantar e/ou andar; existência de plano de saúde privado e tipo de serviço procurado. Pode-se sugerir que essas variáveis não influenciam diretamente a ocorrência da dificuldade na comunicação com os funcionários. Entretanto, é necessário ressaltar que, neste estudo, não foram avaliados o tipo e o grau da deficiência auditiva nem a presença de acompanhantes durante os atendimentos.

As dificuldades relatadas no entendimento do que foi dito por médicos, enfermeiros e outros profissionais dos estabelecimentos de saúde são um fator relevante no que diz respeito à forma e à qualidade dos atendimentos em saúde realizados. A comunicação efetiva é um item imprescindível nesse contexto (TEUTSCH, 2006), pois, além de representar um ponto de interação entre o profissional de saúde e o cliente (SOAR FILHO, 1998), é um importante alicerce para o vínculo de relação entre médico-paciente (BECK et al., 2002) e para a aderência ao tratamento (ZOLNIEREK e DIMATTEO, 2009). Alguns estudos apontam que a comunicação é vista como ferramenta importante da área médica, a ponto de merecer especial ênfase durante a formação acadêmica dos médicos (ROSSI e BATISTA, 2006; GROSSEMAN e STOLL, 2008).

SILVA et al. (2000), nos fornecem os elementos do processo de comunicação. A partir do momento em que o receptor da mensagem transmitida pelo emissor se torna vulnerável e não consegue efetivamente entender o que lhe é dito, o processo de comunicação fica comprometido e, por consequência, a resposta pode não ser a mais adequada ou esperada. Quando esse evento aplica-se à comunicação entre o profissional de saúde e o paciente, fatores como satisfação com o atendimento, aderência ao tratamento, confiança no profissional de saúde, resolução dos sintomas, qualidade de vida, situação de saúde e até mesmo mortalidade podem ser afetados (BECK et al., 2002).

O não entendimento completo de instruções transmitidas por médicos, enfermeiros e outros profissionais de estabelecimentos de saúde não se traduz simplesmente na dificuldade de interpretação do discurso do profissional de saúde, mas em interpretações errôneas que podem acarretar prejuízos ao bem estar e à saúde do indivíduo, comprometendo a acessibilidade aos serviços de saúde. Entende-se por acessibilidade o grau de ajuste entre as características dos serviços de saúde e as das populações, no processo de busca e obtenção da atenção em saúde (FRENK, 1985). Dessa forma, os serviços de saúde usados pelo grupo de pessoas com deficiência auditiva devem ser alvo de intervenções dirigidas e específicas, por tratar-se de um grupo com características diferenciadas, que necessita de intervenções também diferenciadas (FREIRE et al., 2009), para ter assegurado o acesso equitativo aos serviços de saúde garantidos por lei a todos os brasileiros (FERRAZ e VIEIRA, 2009).

Outro fator relevante é a ocorrência da deficiência auditiva com o aumento da idade (CRUZ et al., 2009), considerando-se que o número de pessoas idosas está aumentando de forma acelerada nos países em desenvolvimento (MENDES et al., 2005). Projeções do IBGE (Instituto Brasileiro de Geografia e Estatística) indicam que no período de 2045-2050, a esperança de vida ao nascer no país será de 80,97 
anos contra 72,78 anos registrados em $2008^{\mathrm{W}}$, o que evidencia o envelhecimento populacional. Investimentos direcionados a esse grupo populacional, assim como a avaliação das políticas públicas existentes, poderiam favorecer a qualidade de vida e de saúde dessa parcela da população, garantindo a acessibilidade aos serviços de saúde, diminuindo os obstáculos para obtenção do atendimento adequado em saúde.

De acordo com a Política Nacional de Atenção à Saúde Auditiva (Portaria $n^{\circ}$ 2.073/GM, de 6 de setembro de 2004) (BRASIL, 2004), os resultados das ações decorrentes dessa política na atenção básica e nos serviços de média e alta complexidade devem promover uma ampla cobertura no atendimento às pessoas com deficiência auditiva no Brasil, com aprimoramento da gestão, da disseminação de informações e uma visão dinâmica do estado de saúde dessas pessoas. Além disso, a capacitação e a educação continuada das equipes de saúde em todos os âmbitos de atenção, envolvendo profissionais de diferentes níveis, devem estar alicerçadas nas diretrizes do SUS e em pólos de educação permanente em saúde (MENDES et al., 2005).

A Política Nacional de Saúde da Pessoa com Deficiência (Portaria MS/GM n ${ }^{\circ}$ 1.060 , de 5 de junho de 2002) (BRASIL, 2003) estabelece, como uma de suas diretrizes a "Atenção integral em saúde", atribuindo ao Sistema Único de Saúde (SUS) e à sua rede a responsabilidade direta nos cuidados de saúde das pessoas com deficiências, com garantia de acesso a ações básicas e de maior complexidade, reabilitação e também fornecimento de órteses, próteses e meios auxiliares de locomoção (BRASIL, 2004).

A implementação, avaliação e controle das políticas públicas vigentes pode representar uma ferramenta de gestão e regulação, diminuindo as iniquidades no atendimento em saúde de pessoas com deficiências.

Mais estudos na área são recomendados, pois podem fornecer informações para as políticas de saúde, subsidiando novas formas de planejamento e intervenção.

\section{Conclusão}

As dificuldades na comunicação entre pacientes e profissionais de saúde relatadas neste estudo, podem implicar em menor utilização dos serviços de saúde, comprometendo a qualidade de vida e de saúde das pessoas com deficiência auditiva, além de influenciar na relação médico-paciente, na confiança no profissional de saúde e na aderência ao tratamento.

A acessibilidade ao serviço de saúde pode ser um problema para as pessoas com algum tipo de deficiência auditiva. Intervenções específicas e dirigidas junto aos serviços de saúde utilizados por esse grupo populacional, assim como a avaliação das políticas públicas vigentes, efetivando o que é garantido por lei, representariam uma forma de minimizar as dificuldades decorrentes dessa deficiência e promover a atenção integral em saúde.

O preparo das equipes de saúde também é um fator essencial para o encaminhamento das pessoas com deficiência auditiva aos serviços de média e alta complexidade, para casos de avaliação audiológica, protetização e reabilitação auditiva. Além disso, a capacitação de profissionais de saúde para atuar em saúde

\footnotetext{
${ }^{\mathrm{W}}$ IBGE - Projeção da população do Brasil por sexo e idade - 1980-2050. Disponível em: http://www.ibge.gov.br/home/estatistica/populacao/projecao_da_populacao/2008/projecao.pdf?bcsi_s can_D2AD62A071FDB58E=0\&bcsi_scan_filename=projecao.pdf, Acessado em 2009 set 04.
} 
auditiva pode levar a impactos positivos entre os profissionais e também entre os gestores, tanto no conhecimento sobre o tema quanto no acesso dos deficientes auditivos aos serviços de audiologia (MELO e ALVARENGA, 2009). Dessa forma, estariam garantidos os princípios de universalidade, equidade e integralidade do Sistema Único de Saúde (SUS).

\section{Bibliografia}

Beck RS, Daughtridge R, Sloane P. Physician-patient communication in the primary care office: a systematic review. J Am Board Fam Pract 2002; 15: 25-38. Bernardes LCG, Maior IMML, Spezia CH, Araujo TCCF. Pessoas com deficiência e políticas de saúde no Brasil: reflexões bioéticas. Ciência\&Saúde Coletiva 2009; 14(1): 31-8.

Brasil. Ministério da Saúde. Política Nacional de Saúde da Pessoa Portadora de Deficiência. Brasília: Ministério da Saúde; 2003.

Brasil. Ministério da Saúde. Portaria GM/MS nº 198 de 13 de fevereiro de 2004. Institui a Política nacional de educação permanente em saúde como estratégia do Sistema Único de Saúde para formação e desenvolvimento de trabalhadores para o setor e dá outras providências. Diário Oficial da Republica Federativa do Brasil. 29 fev 2004.

Cardoso AHA, Rodrigues KG, Bachion MM. Perception of persons with severe or profound deafness about the communication process during health care. Rev. Latino-Am. Enfermagem 2006; 14(4): 553-560.

Castro SS, César CLG, Carandina L, Barros MBA, Alves MCGP, Goldbaum M. Deficiência visual, auditiva e física: prevalência e fatores associados em estudo de base populacional. Cad. Saúde Pública 2008; 24(8): 1773-1782.

Cruz MS, Oliveira LR, Carandina L, Lima MCP, Cesar CLG, Barros MBA, Alves MCGP, Goldbaum M. Prevalência de deficiência auditiva referida e causas atribuídas: um estudo de base populacional. Cad Saude Púb. 2009; 25(5): 112331.

Cruz MS, Oliveira LR, Carandina L. Inquéritos de saúde e fonoaudiologia. Rev CEFAC 2009; 11(1): 166-72.

Ferraz OLM, Vieira FS. Direito à saúde, recursos escassos e equidade: os riscos da interpretação judicial dominante. Dados 2009; 52(1): 223-251.

Freire DB,Gigante LP, Béria JU, Palazzo LS, Figueiredo ACL, Raymann BCW. Acesso de pessoas deficientes auditivas a serviços de saúde em cidade do Sul do Brasil. Cad Saude Pública 2009; 25(4): 889-97.

Frenk J. El concepto y la medición de accesibilidad. Salud Pública de México, 1985; 27(5): 438-53.

Grosseman S, Stoll C. O ensino-aprendizagem da relação médico-paciente: estudo de caso com estudantes do último semestre do curso de medicina. Rev Bras Educ Med 2008; 32(3):301-8.

IBGE - Instituto Brasileiro de Geografia e Estatística. Censo Demográfico 2000. Características Gerais da População. Resultados da Amostra. Rio de Janeiro: IBGE; 2003.

Iezzoni LI, O'Day BL, Killeen M, Harker H. Communicating about health care: observations from persons who are deaf or hard of hearing. Ann Intern Med. 2004; 140(5):356-62. 
Jacob LCB, Alvarenga KF, Zeigelboim BS. Avaliação audiológica do sistema nervoso auditivo central. Arquiv. Int Otorr 2000; 4(4): 144-151.

Marques ACO, Kozlowski L, Marques JM. Reabilitação auditiva no idoso. Rev. Bras. Otorrinolaringol. 2004; 70: 806-11.

Melo TM, Alvarenga KF. Capacitação de profissionais da saúde na área de saúde auditiva: revisão sistemática. Rev. soc. bras. fonoaudiol. 2009; 14(2): 280-286.

McAleer M. Communicating effectively with deaf patients. Nurs Stand. 2006; 20(19): 51-4.

Mendes MRSSB, Gusmão JL, Faro ACM, Leite RCBO. A situação social do idoso no Brasil: uma breve consideração. Acta Paul. Enferm. 2005; 18(4): 422426.

Organização das Nações Unidas. Follow-up to the World Summit for Social Development and the twenty-fourth special session of the General Assembly: emerging issues:"Mainstreaming disability in the development agenda". 2008. [acessado em 2009 set 07]. Disponível em: http://www.un.org/disabilities/documents/reports/e-cn5-2008-6.doc.

Pagliuca LMF, Fiúza NLG, Rebouças CBA. Aspectos da comunicação da enfermeira com o deficiente auditivo. Rev Esc Enferm USP 2007; 41(3): 411-8.

Pereira PC, Fortes PA. Communication and information barriers to health assistance for deaf patients. Am Ann Deaf. 2010;155(1):31-7.

Rossi PS, Batista NA. O ensino da comunicação na graduação em Medicina, uma abordagem. Interface 2006; 10(19): 93-102.

Silva ALA, Guilherme M, Rocha SSL, Silva MJP. Comunicação e enfermagem em saúde mental - reflexões teóricas. Rev Latino-am. Enfermagem 2000; 8(5): 65-70.

Soar Filho EJ. A interação médico-cliente. Rev Ass Med Brasil 1998; 44(1): 3542.

Teutsch C, Patient-doctor communication. Med Clin N Am 2006; 87:1115-45.

Zolnierek KBH, DiMatteo MR. Physician communication and patient adherence to treatment: a meta-analysis. Med Care 2009; 47: 826-34. 
Quadro 1: Caracterização do grupo de pessoas com deficiência estudado, AceSS, 2007.

\begin{tabular}{|c|c|c|}
\hline Variáveis estudadas & $\mathbf{n}$ & $\%$ \\
\hline Problemas para ouvir e entender o que os funcionários disseram ${ }^{(1)}$ & 160 & 100,00 \\
\hline 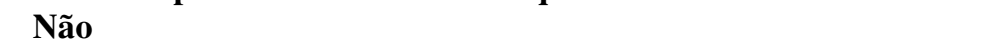 & 104 & 65,00 \\
\hline Sim & 56 & 35,00 \\
\hline Deficiência & 160 & 100,00 \\
\hline Auditiva & 95 & 59,38 \\
\hline Múltipla & 65 & 40,62 \\
\hline Sexo & 160 & 100,00 \\
\hline $\mathbf{M}$ & 85 & 53,13 \\
\hline $\mathbf{F}$ & 75 & 46,87 \\
\hline Idade (anos) & 160 & 100,00 \\
\hline $14-54$ & 28 & 17,50 \\
\hline 55-69 & 40 & 25,00 \\
\hline 70-77 & 43 & 26,88 \\
\hline 78-97 & 49 & 30,62 \\
\hline Renda do chefe da Família ${ }^{(2)}$ & 160 & 100,00 \\
\hline$<1$ & 101 & 63,13 \\
\hline 1 & 20 & 12,50 \\
\hline $2-4,99$ & 20 & 12,50 \\
\hline $5-7,99$ & 8 & 5,00 \\
\hline$>8$ & 11 & 6,87 \\
\hline $\operatorname{Raça}^{(3)}$ & 159 & 100,00 \\
\hline Branca & 96 & 60,38 \\
\hline Negro/Mulato & 56 & 35,22 \\
\hline Outros & 7 & 4,40 \\
\hline Necessidade de ajuda ${ }^{(4)}$ & 160 & 100,00 \\
\hline Não & 142 & 88,75 \\
\hline Sim & 18 & 11,28 \\
\hline Plano de saúde privado & 160 & 100,00 \\
\hline Não & 99 & 61,88 \\
\hline Sim & 61 & 38,12 \\
\hline Serviço procurado ${ }^{(5)}$ & 159 & 100,00 \\
\hline UBS/AMA/APA/ESF/PSF & 59 & 37,11 \\
\hline Consultório médico & 34 & 21,38 \\
\hline Ambulatório & 14 & 8,81 \\
\hline Emergência & 10 & 6,29 \\
\hline Hospital & 37 & 23,27 \\
\hline Outros & 5 & 3,14 \\
\hline
\end{tabular}

Notas:

\footnotetext{
${ }^{(1)}$ Médicos, enfermeiras, recepcionistas, atendentes e outros funcionários

${ }^{(2)}$ Salários mínimos por mês

(3) 1 perda.

(4) Ajuda para tomar banho e vestir-se, comer, levantar e/ou andar.

(5) 1 perda.
} 


\begin{tabular}{|c|c|c|c|c|c|c|}
\hline \multirow{3}{*}{ Variáveis } & \multicolumn{4}{|c|}{$\begin{array}{c}\text { Problemas para ouvir e entender o que os } \\
\text { funcionários disseram }\end{array}$} & \multirow{2}{*}{\multicolumn{2}{|c|}{ Total }} \\
\hline & \multicolumn{2}{|c|}{ Não } & \multicolumn{2}{|c|}{ Sim } & & \\
\hline & $\mathrm{n}(\mathbf{1 0 4})$ & $\%(65,00)$ & $\mathrm{n}(56)$ & $\%(35,00)$ & $\mathrm{n}(160)$ & $\%(100,00)$ \\
\hline \multicolumn{7}{|l|}{ Deficiência } \\
\hline Auditiva & 62 & 65,26 & 33 & 34,74 & 95 & 100,0 \\
\hline Múltipla & 42 & 64,62 & 23 & 35,38 & 65 & 100,0 \\
\hline \multicolumn{7}{|l|}{ Sexo } \\
\hline $\mathbf{M}$ & 55 & 64,71 & 30 & 35,29 & 85 & 100,0 \\
\hline $\mathbf{F}$ & 49 & 65,33 & 26 & 34,67 & 75 & 100,0 \\
\hline \multicolumn{7}{|l|}{ Idade (anos) } \\
\hline 14-54 & 20 & 71,43 & 8 & 28,57 & 28 & 100,0 \\
\hline $55-69$ & 26 & 65,00 & 14 & 35,00 & 40 & 100,0 \\
\hline 70-77 & 27 & 62,79 & 16 & 37,21 & 43 & 100,0 \\
\hline 78-97 & 31 & 63,27 & 18 & 36,73 & 49 & 100,0 \\
\hline \multicolumn{7}{|l|}{ Renda do chefe da } \\
\hline \multicolumn{7}{|l|}{ Família $^{(2)}$} \\
\hline$<1$ & 67 & 66,34 & 34 & 33,66 & 101 & 100,0 \\
\hline 1 & 10 & 50,00 & 10 & 50,00 & 20 & 100,0 \\
\hline 2-4,99 & 13 & 65,00 & 7 & 35,00 & 20 & 100,0 \\
\hline $5-7,99$ & 6 & 75,00 & 2 & 25,00 & 8 & 100,0 \\
\hline$>8$ & 8 & 72,73 & 3 & 27,27 & 11 & 100,0 \\
\hline \multicolumn{7}{|l|}{$\operatorname{Raça}^{(3)}$} \\
\hline Branca & 62 & 64,58 & 34 & 35,42 & 96 & 100,0 \\
\hline Negro/Mulato & 36 & 64,29 & 20 & 35,71 & 56 & 100,0 \\
\hline Outros & 5 & 71,43 & 2 & 28,57 & 7 & 100,0 \\
\hline \multicolumn{7}{|l|}{ Necessidade de ajuda ${ }^{(4)}$} \\
\hline Não & 26 & 72,22 & 10 & 27,78 & 36 & 100,0 \\
\hline Sim & 78 & 62,90 & 46 & 37,10 & 124 & 100,0 \\
\hline \multicolumn{7}{|l|}{ Plano de saúde privado } \\
\hline Não & 93 & 65,49 & 49 & 34,51 & 142 & 100,0 \\
\hline Sim & 11 & 61,11 & 7 & 38,89 & 18 & 100,0 \\
\hline \multicolumn{7}{|l|}{ Serviço procurado ${ }^{(5)}$} \\
\hline UBS/AMA/APA/ESF/ & 41 & 69,49 & 18 & 30,51 & 59 & 100,00 \\
\hline \multicolumn{7}{|l|}{ PSF } \\
\hline Consultório médico & 26 & 76,47 & 8 & 23,53 & 34 & 100,00 \\
\hline Ambulatório & 7 & 50,00 & 7 & 50,00 & 14 & 100,00 \\
\hline Emergência & 5 & 50,00 & 5 & 50,00 & 10 & 100,00 \\
\hline Hospital & 21 & 56,76 & 16 & 43,24 & 37 & 100,00 \\
\hline Outros & 4 & 80,00 & 1 & 20,00 & 5 & 100,00 \\
\hline \multicolumn{7}{|l|}{ Notas: } \\
\hline $\begin{array}{l}{ }^{(1)} \text { Médicos, enfermeiras, recepci } \\
\text { (2) Salários mínimos por mês. } \\
\text { (3) } 1 \text { perda. } \\
{ }^{(4)} \text { Ajuda para tomar banho e vest } \\
{ }^{(5)} 1 \text { perda. }\end{array}$ & ss, aten & outros fun & & & & \\
\hline
\end{tabular}




\section{CONSIDERAÇÕES FINAIS}

Algumas observações sobre as limitações deste estudo devem ser feitas. Todas as informações foram auto-referidas, sem confirmações por exames físicos ou clínicos, o que melhoraria a exatidão dos dados, porém, com custos financeiros agregados. Os níveis de funcionalidade poderiam ter sido avaliados por instrumentos específicos. Diferentes níveis de funcionalidade implicam em diferentes resultados ou formas de abordagem durante a pesquisa e análise dos dados. Estudos separados deveriam ser feitos para cada uma das deficiências. Quando diferentes tipos de deficiência são abordados em uma mesma pesquisa, problemas de definição e coleta de dados podem ser encontrados. O tempo de deficiência deve ser coletado quando se estuda as pessoas com alguma deficiência, considerando que essa variável pode ter influência sobre outras, como a autoavaliação em saúde e o aparecimento de doenças crônicas. Além disso, os estudos transversais permitem apenas a identificação de uma relação entre as variáveis estudadas, mas a direção dessa associação é determinada por outros desenhos de estudo.

Por questões operacionais, optamos por apresentar, nesta tese, as informações de três artigos que já foram preparados para publicação. Entretanto, outros dados, que foram coletados e analisados, serão oportunamente submetidos à publicação. Para efeitos da defesa de doutorado, somente os achados dos três artigos foram apresentados e discutidos.

No primeiro manuscrito, as análises mostraram que o tempo de deslocamento até o serviço de saúde, a necessidade de acompanhante e o meio de transporte utilizado foram fatores importantes envolvidos no processo de deslocamento da casa do entrevistado até o serviço de saúde, segundo os relatos das pessoas com deficiência participantes da Fase I do Estudo AceSS. O tempo de espera para atendimento, problemas com estacionamento, ausência de rampas, falta de sanitários adaptados, sala de espera com lugares insuficientes, inexistência de cadeira de rodas para pacientes e salas bloqueadas por obstáculos arquitetônicos foram os fatores citados pelos entrevistados como problemas na acessibilidade aos serviços de saúde, dificultando ou impedindo o seu correto uso. Os problemas citados anteriormente atuariam como obstáculos ao uso dos serviços de saúde pelas pessoas com 
deficiência, contrariando o princípio da equidade, que norteia o Sistema Único de Saúde (SUS). A equidade em saúde se baseia na idéia de que todos os indivíduos de uma sociedade devem ter oportunidades iguais para desenvolver seu potencial de saúde e o sistema que se baseia na equidade deveria atuar contra os obstáculos observados, reduzindo as diferenças evitáveis ou injustas entre os indivíduos.

No segundo manuscrito, $15,92 \%$ dos entrevistados relataram enfrentar problemas de acessibilidade aos serviços de saúde. O artigo também mostrou que as pessoas com paralisia ou amputação e com mais de uma deficiência tiveram mais problemas de acessibilidade que os outros, assim como pessoas com idade inferior a 78 anos e aquelas que precisavam de ajuda para ir ao serviço de saúde.

O terceiro manuscrito mostrou que 35\% dos 160 entrevistados com alguma deficiência auditiva relataram problemas para ouvir e entender o que foi dito pelos profissionais de saúde. Esse fato pode implicar em menor utilização dos serviços de saúde e possíveis prejuízos à saúde das pessoas com esse tipo de deficiência.

As informações sobre os tipos de problemas na acessibilidade aos serviços de saúde, bem como do perfil das pessoas com deficiência que enfrentam esses problemas, podem ser úteis na criação de programas e políticas em saúde que tenham por objetivo o incremento do uso dos serviços de saúde por esse grupo populacional. O aumento do uso e o uso efetivo dos serviços de saúde poderiam resultar em melhoria na qualidade de vida dessas pessoas. 


\section{BIBLIOGRAFIA}

Ameratunga SN; Norton RN; Benett DA; Jackson RT. Risk of disability due to car crashes: a review of the literature and methodological issues. Injury 2004; 35(11): 116-27.

Ameratunga SN; Norton RN; Connor JL; Robinson E; Civil I; Covardale J; Bennett D; Jackson RT. A population-based cohort study of longer-term changes in health of car drivers involved in serious crashes. Ann Emerg Med. 2006; 48(6): 729-36.

Amiralian MLT; Pinto EB; Ghirardi MIG; Lichtig I; Masini EFS; Pasqualin L. Conceituando deficiência. Rev. Saúde Pública 2000; 34(1): 97-103.

Amorim VMSL; Barros MBA; Cesar CLG; Carandina L; Goldbaum M. Fatores associados à não realização do exame de Papanicolaou: um estudo de base populacional no Município de Campinas, São Paulo, Brasil. Cad de Saú Púb, 2006; 22(11): 2329-2338.

Andrade SC. Qualidade da dieta de adolescentes residentes na região metropolitana de São Paulo, 2000- 2002. São Paulo; 2004. [Projeto de Mestrado - Faculdade de Saúde Pública da Universidade de São Paulo].

Andrade SC; Barros MBA; Carandina L; Goldbaum M; Cesar CLG; Fisberg RM. Dietary quality and associated factors among adolescents of the State of Sao Paulo, Brazil. The Journal of Pediatrics 2010; 156: 456-460.

Barros AJ; Hirakata VN. Alternatives for logistic regression in cross-sectional studies: an empirical comparison of models that directly estimate the prevalence ratio. BMC Med Res Methodol. 2003; 3:21.

Beange H. Caring for a vulnerable population. The Medical Journal of Australia, 1996; 164: 159-60.

Beatty PW; Hagglund KJ; Neri MT; Dhont KR; Clarck MJ; Hilton AS. Access to health care services among people with chronic or disabling conditions: patterns and predictors. Arch Phys Med Rehabil, 2003; 84(10): 1417-25.

Branigan M; Stewart DE; Tardif GS; Veltman A. Perceptions of primary healthcare services among persons with physical disabilities. Part 2: Quality issues. Medscape Gen Med, 2001; 3(2).

Brasil.Constituição da República Federativa do Brasil. Brasília, Senado Federal, Centro Gráfico, 1988.

Brasil. Decreto $\mathrm{n}^{\mathrm{o}} 3.298$, de 20 de dezembro de 1999. Política Nacional para Integração da Pessoa Portadora de Deficiência. Brasília-DF, 20 de dezembro de 1999.

Brasil. Decreto n. 5.296, de 2 de dezembro 2004. Regulamenta as Leis n.10.048, de 8 de novembro de 2000, que dá prioridade de atendimento às pessoas que especifica, e n. 10.098 , de 19 de dezembro de 2000, que estabelece normas gerais e critérios básicos para a promoção da acessibilidade das pessoas portadoras de deficiência ou 
com mobilidade reduzida, e da outras providências. Diário Oficial da União, Brasília, 3 dez. 2004. Seção 1, p. 5.

Brasil. Convenção sobre os Direitos das Pessoas com Deficiência. Brasília: 2007.

Bueno MB. Consumo alimentar de açúcar adicionado entre adultos residentes no Estado de São Paulo. São Paulo; 2004. [Tese de qualificação de Doutorado Faculdade de Saúde Pública da USP].

Bueno MB; Cesar CLG; Martini LA; Fisberg RM. Dietary calcium intake and overweight: An epidemiologic view. Nutrition 2008; 24: 1110-1115.

Campos FG; Barrozo LV; Ruiz T; Cesar CLG; Barros MBA; Carandina L; Goldbaum M. Distribuição espacial dos idosos de um município de médio porte do interior paulista segundo algumas características sócio-demográficas e de morbidade. Cadernos de Saúde Pública 2009; 25: 77-86.

Carvalho WO. Uso de serviços de saúde materno-infantis na Região Sudoeste da Grande São Paulo em dois períodos: 1990-2002. Inquérito de saúde como fonte de dados para estudos de utilização de serviços de saúde. São Paulo; 2004. [Tese de Doutorado - Faculdade de Saúde Pública da USP].

Carvalho WO; Cesar CLG; Carandina L; Barros MBA; Alves MCGP; Goldbaum M. Inquéritos de saúde e uso de serviços de saúde em três municípios da Grande São Paulo. Revista Panamericana de Salud Pública / Pan American Journal of Public Health ,2008; 24: 314-323.

Castro SS; Cesar CLG; Carandina L; Barros MBA; Alves MCGP; Goldbaum M. Deficiência visual, auditiva e física: prevalência e fatores associados em estudo de base populacional. Cadernos de Saúde Pública 2008; 24: 1773-1782.

Castro SS, Cesar CL, Carandina L, Barros MB, Alves MC, Goldbaum M. Physical disability, recent illnesses and health self-assessment in a population-based study in São Paulo, Brazil. Disabil Rehabil. 2010 [Epub ahead of print]

Cesar CLG; Carandina L; Alves MCP; Barros MBA; Goldbaum M. Saúde e Condição de Vida em São Paulo - Inquérito Multicêntrico de Saúde no Estado de São Paulo - ISA-SP. São Paulo: USP/FSP, 2005.

Chan L; Doctor JN; Maclehose RF; Lawson H; Rosenblatt RA; Baldwin LM; Jha A. Do Medicare patients with disabilities receive preventive services? A populationbased study. Arch Phys Med Rehabil, 1999; 80: 642-6.

Chan L; Beaver S; Maclehose RF; Jha A; Maciejewski M; Doctor JN. Disability and health care costs in the medicare population. Arch Phys Med Rehabil, 2002; 83: 1196-1201.

Chaves ES; Boninger ML; Cooper R; Fitzgerald SG; Gray DB; Cooper RA. Assessing the influence of wheelchair technology on perception of participants in spinal cord injury. Arch Phys Med Rehabil, 2004; 85: 1854-8.

Christensen LB; Hede B; Petersen PE. Public dental health care program for persons with disability. Acta Odontologica Scandinavica, 2005; 63: 278-83. 
Coppel DH; Packham CJ; Varnam MA. Providing welfare tights advice in primary care. Public Health, 1999; 133: 131-35.

Coutinho LMS; Scazufca M; Menezes PR. Métodos para estimar razão de prevalência em estudos de corte transversal. Rev. Saúde Pública 2008; 42(6): 992998.

Coyle CP; Santiago MC; Shank JW; Ma GX; Boyd R. Secondary conditions and women with physical disabilities: a descriptive study. Arch Phys Med Rehabil, 2000; 81: 1380-7.

Cruz MS; Oliveira LR; Carandina L; Lima MCP; Cesar CLG; Barros MBA; Alves MCGP; Goldbaum M. Prevalência de deficiência auditiva referida e causas atribuídas: um estudo de base populacional. Cadernos de Saúde Pública 2009; 25: 1123-1131.

Cunha ABO; Vieira-da-Silva LM. Acessibilidade aos serviços de saúde em um município do Estado da Bahia, Brasil, em gestão plena do sistema. Cad. Saúde Pública 2010; 26(4): 725-73.

Currie DM. Primary care for people with disabilities: the physiatrist's perspective. American Journal of Physical Medicine and Rehabilitation, 1997; 76(3S): 25-9.

Cusick CP; Brooks CA; Whiteneck GG. The use of proxies in community integration research. Arch Phys Med Rehabil, 2001; 82: 1018-24.

Dejong G. Primary care for people with disabilities: an overview of the problem. American Journal of Physical Medicine and Rehabilitation, 1997; 76(3S): 2-8.

Detels R; Mcewen J; Beaglehole R; Tanaka H. Oxford Textbook of Public Health. New York: Oxford University Press; 2004.

Di Nubila HBV; Buchalla CM. O papel das Classificações da OMS - CID e CIF nas definições de deficiência e incapacidade. Rev. bras. Epidemiol. 2008; 11(2): 324335 .

Diab ME; Johnston MV. Relationships between level of disability and receipt of preventive health services. Arch Phys Med Rehabil, 2004; 85(5): 749-57.

Edwards DM; Merry AJ. Disability part 2: access to dental services for disabled people. A questionnaire survey of dental practices in Merseyside. British Dental Journal, 2002; 193(5): 253-5.

Fänge A; Iwarsson S; Persson A. Accessibility to the public environment as perceived by teenagers with functional limitations in a south Swedish town centre. Disabil Rehabil, 2002; 24(6): 318-26.

Fernandez PMF. Consumo alimentar de adolescentes da região metropolitana de São Paulo. São Paulo; 2004. São Paulo; 2004. [Tese de qualificação de Mestrado Faculdade de Saúde Pública da USP].

Fisberg RM. A qualidade da dieta e seus fatores associados em adultos residentes no Estado de São Paulo. São Paulo; 2005 [Tese de Livre Docência - Faculdade de Saúde Pública da USP] 
Fisberg RM; Slater B; Barros RR; Lima FD; Cesar CLG; Carandina L; Barros MBA; Goldbaum M. Healthy Eating Index: evaluation of adapted version and its applicability. Rev. Nutr. 2004; 17(3): 301-318.

Florindo AA; Guimarães W; Cesar CLG; Barros MBA; Alves MCGP; Goldbaum M. Epidemiology of leisure, transportation, occupational and household physical activity: prevalence and associated factors. Journal of Physical Activity \& Health 2009; 6: 625-32.

Fouts BS; Andersen E; Hagglund K. Disability and satisfaction with access to health care. Journal of Epidemiology and Community Health, 2000; 54: 770-1.

França ISX; Pagliuca LMF. Inclusão social da pessoa com deficiência: conquistas, desafios e implicações para a enfermagem. Rev. esc. enferm. USP 2009; 43(1): 17818.

Francisco PMSB; Donalisio MR; Barros MBA; Cesar CLG, Carandina L; Goldbaum M. Fatores Associados à Vacinação Contra a Influenza em Idosos. Revista Panam Salud Publica (Panam. J. Public Healt), 2006; 19(4): 259-264.

Francisco PMSB; Donalisio MR; Barros MBA; Cesar CLG; Carandina L; Goldbaum M. Medidas de associação em estudo transversal com delineamento complexo: razão de chances e razão de prevalência. Revista Brasileira de Epidemiologia 2008; 11: 347-355.

Francisco PM; Belon AP; Barros MBA; Carandina L; Alves MCGP; Goldbaum M; Cesar CLG. Diabetes auto-referido em idosos: prevalência, fatores associados e práticas de controle. Cadernos de Saúde Pública 2010; 26: 175-184.

Frenk J. El concepto y la medición de accesibilidad. Salud Pública de México, 1985; 27(5): 438-53.

Galvanese GCSM. Perfil de morbidade referida e aspectos do estilo de vida de adolescentes de 12 a 19 anos da Grande São Paulo (Estudo de Base Populacional). São Paulo; 2004. [Tese de qualificação de Doutorado - Faculdade de Saúde Pública da USP].

Godoy FC. Índice de Qualidade da Dieta de adolescentes residentes no Distrito do Butantã, município de São Paulo, SP. São Paulo; 2004. [Tese de qualificação de Mestrado - Faculdade de Saúde Pública da USP].

Goodal CJ. Is disability any business of nurse education? Nurs Ed Today, 1995; 15(5): 323-7.

Grabois EW; Nosck MA; Rossid D. Accessibility of primary care physician's offices for people with disabilities. An analysis of compliance with the Americans With Disabilities Act. Arch Fam Med., 1999; 8: 44-51.

Gray DB; Gould M; Bickenbach JE. Environmental barriers and disability. J of Arch and Plann Res, 2003; 20(1): 29-37.

Grönvik L. The fuzzy buzz word: conceptualizations of disability in disability research classics. Soc Helt\&Illness, 2007; 29(5): 750-66. 
Guimarães VMV. Atividade física e hipertensão arterial na Grande São Paulo, 2002: um estudo de base populacional. São Paulo; 2003a. [Dissertação de Mestrado Faculdade de Saúde Pública da USP].

Guimarães VMV. Desigualdades em saúde do adulto: alguns aspectos do estilo de vida. São Paulo; 2003b. [Projeto para Exame de Qualificação de Doutorado Faculdade de Saúde Pública da USP].

Harmer L. Health care delivery and deaf people: practice, problems, and recommendations for change. Journal of Deaf Studies and Deaf Education, 1999; 4(2): 73-109.

Harrison T. Health promotion for persons with disabilities. What does the literature reveal? Family and Community Health, 2006; 29(1S): 12S-19S.

Iezzoni LI; Davis RB; Soukup J; O’Day B. Satisfaction with quality and access to health care among people with disabling conditions. International Journal for Quality in Health Care, 2002; 14(5): 369-81.

Iezzoni LI; O’Day BL; Killen M; Harker H. Communicating about health care: observations from persons who are deaf or hard of hearing. Ann Intern Med, 2004; 140(5): 356-62.

Iwarsson S; Stahl A. Accessibility, usability and universal design - positioning and definition of concepts describing person-environment relationships. Disability and Rehabilitation, 2003; 25(2): 57-66.

Jones KE; Tamari IE. Making our offices universally accessible: guidelines for physicians. Can Med Assoc J., 1997; 156(5): 647-56.

Kersten P; George S; Mclellan L; Smith JAE; Mullee MA. Met and unmet needs reported by severely disabled people in Southern England. Disabil Rehabil, 2000; 22(16): 737-44.

Kroll T; Jones GC; Kehn M; Neri MT. Barriers and strategies affecting the utilization of primary preventive services for people with physical disabilities: a qualitative inquiry. Health an Soc Care Comm, 2006; 14(4): 284-93.

Lawthers AG; Pransky GS; Petrson LE; Himmelstein JH. Rethinking quality in the context of persons with disability. Int. Journal for Qual. In Health Care, 2003; 15(4): 289-99.

Lefèvre F; Lefèvre AMC. O discurso do sujeito coletivo: um novo enfoque em pesquisa qualitativa (desdobramentos). Caixas do Sul. Ed. Diálogo, 2 edição; 2003.

Lefèvre F; Lefèvre AMC. Depoimentos e discursos, uma proposta de análise em pesquisa social. Brasília. Ed. Liber Livro; 2005.

Lefèvre F; Lefèvre AMC. The collective subject that speaks. Interface, 2006; 10(20): 517-524.

Lianza S. Medicina de Reabilitação. São Paulo. Ed Guabara-Koogan, $2^{\text {a }}$ Edição;1995.

Lima MCP; Menezes PR; Carandina L; Cesar CLG; Barros MBA; Goldbaum M. Transtornos mentais comuns e uso de psicofármacos: impacto das condições 
socioeconômicas. Revista de Saúde Pública / Journal of Public Health 2008; 42: 717723.

Lima MG; Barros MBA; Cesar CLG; Goldbaum M; Carandina L; Ciconelli RM. Elderly health related quality of life: SF-36 evaluation in a population-based study. Cadernos de Saúde Pública 2009a; 25: 2159-2167.

Lima MG; Barros MBA; Cesar CLG; Goldbaum M; Carandina; Ciconelli RM. Impact of chronic disease on quality of life among the elderly in the state of São Paulo, Brazil: a populartion-based study. Revista Panamericana de Salud Pública / Pan American Journal of Public Health 2009b; 25: 314-321.

Lin JD; Wu JL; Lee PN. Healthcare needs of people with intellectual disability in institutions in Taiwan: outpatient care utilization and implications. Journal of Intellectual Disability Research, 2003; 47(3): 169-80.

Lipson JG; Rogers JG. Cultural aspects of disability. J. Transcultural Nursing, 2000; 11(3): 212-9.

Lollar DJ, Crews JE. Redefining the role of public health in disability. Ann Rev Pub Health, 2003; 24: 195-208.

Lutz BJ, Bowers BJ. Disability in everyday life. Qual Health Res., 2005; 15(8): 1037-54.

Magnabosco C. A população de cães e gatos nos domicílios e o risco potencial para a saúde humana analisado através da utilização de um inquérito domiciliar de saúde no Município de São Paulo. São Paulo; 2005. [Tese de Mestrado - Faculdade de Saúde Pública da Universidade de São Paulo].

Maragno L; Goldbaum M; Gianini RJ; Novaes HMD; Cesar CLG. Prevalência de transtornos mentais comuns em populações atendidas pelo Programa Saúde da Família (QUALIS) no Município de São Paulo, Brasil. Cad de Saú Púb, 2006; 22(8): 1639-1648.

McAlpine DD; Mechanic D. Utilization of specialty mental health care among persons with severe mental illness: the roles of demographics, need, insurance, and risk. Health Service Research, 2000; 35(1): 277-92.

Merrell J, Kinsella F, Murphy F, Philpin S, Ali A. Accessibility and equity of health and social care services: exploring the views and experiences of Bangladeshi carers in South Wales, UK. Health Soc Care Community. 2006; 14(3): 197-205.

Morimoto JM. Fatores associados à qualidade da dieta de adultos residentes na região metropolitana de São Paulo. São Paulo; 2005. [Dissertação de Mestrado Faculdade de Saúde Pública da Universidade de São Paulo].

Morimoto JM; Latorre MRDO; Cesar CLG; Carandina L; Barros MBA; Goldbaum M; Fisberg RM. Fatores associados à qualidade da dieta de adultos residentes na região Metropolitana de São Paulo, Brasil, 2002. Cadernos de Saúde Pública 2008; 24: 169-178.

Neri MT; Kroll T. Understanding the consequences of access barriers to health care: experiences of adults with disabilities. Disability and Rehabilitation, 2003; 25(2): 8596. 
Nosek MA; Hughes RB; Petersen NJ; Taylor HB; Robinson-Whelen S; Byrne M; Morgan R. Secondary conditions in a community-based sample of women with physical disabilities over a 1-year period. Arch Phys Med Rehabil, 2006; 87(3): 3207.

Odette F; Yoshida KK; Israel P; Li A; Ullman D; Colontonio A; Maclean H; Locker D. Barriers to wellness activities for Canadian women with physical disabilities. Health Care for Women International, 2003; 24(2): 125-134.

OMS, Organização Mundial de Saúde. CIF: Classificação Intenacional de Funcionalidade, Incapacidade e Saúde [Centro Colaborador da Organização Mundial de Saúde para a família de classificações internacionais, org.; Coordenação da tradução Cássia Maria Buchalla]. - São Paulo: Editora da Universidade de São Paulo, 2003.

Paternez ACAC. Consumo alimentar de açúcar adicionado em adolescentes residentes no Estado de São Paulo. São Paulo; 2004. [Tese de qualificação de Doutorado - Faculdade de Saúde Pública da USP].

Peat M. Attitudes and access: advancing the rights of people with disabilities. Canadian Med Assoc J, 1997; 156(5): 657-9.

Pelicioni AF. Padrão de consumo de medicamentos em duas áreas da Região Metropolitana de São Paulo, 2001-2002. São Paulo; 2005. [Tese de Mestrado Faculdade de Saúde Pública da Universidade de São Paulo].

Ravesloot C; Seekins T; Walsh J. A structural analysis of secondary conditions experienced by people with physical disabilities. Rehabilitation and Psychology, 1997; 42(1): 3-16.

Ravesloot C; Seekins; White G. Living well with a disability health promotion intervention: improved health status for consumers and lower costs for health care policymakers. Rehabilitation Psychology, 2005; 50(3): 239-45.

Ricketts TC; Goldsmith LJ. Access in health services research: the battle of the frameworks. Nurs Out, 2005; 53(6): 274-80.

Rocha EF. Reabilitação de pessoas com deficiência. São Paulo, Roca, 2006.

Rose ML. Deaf and Dumb in ancient Greece. In: Davis LJ. The disability studies reader. $2^{\mathrm{a} E d}$. New York, Taylor\&Francis Group, 2006.

Rosenbach ML. Access and satisfaction within the disabled Medicare population. (Access to health services for vulnerable populations). Health Care Financing Review, 1995; 17(2): 147-57.

Schopp LH; Sanford TC; Hagglund KJ; Gay JW; Coatney MA. Removing service barriers for women with physical disabilities: promoting accessibility in the gynecologic care setting. J Midwifery \& Women’s Health, 2002; 47(2): 74-9.

Seekins T; Clay J; Raversloot C. A descriptive study of secondary conditions reported by a population of adults with physical disabilities served by three independent living centers in a rural state. The Journal of Rehabilitation, 1994; 60(2): 47-52. 
Seullion P. Conceptualizing disability in nursing: some evidence from students and their teachers. J Adv Nurs., 1999; 29(3): 648-57.

Shakespeare T, Walson N. Defending the social model. Disabil Soc., 1997; 12(2):293-300.

Shapiro J; Mosqueda L; Botros D. A caring partnership. Expectations of ageing persons with disabilities for their primary care doctors. Family Practice, 2003; 20(6): 635-41.

Smeltzer SC. Improving the health and wellness of persons with disabilities: a call to action too important for nursing to ignore. Nur Out., 2007; 55(4): 189-95.

SMPED, Secretaria Municipal da Pessoa com Deficiência e Mobilidade Reduzida Prefeitura de São Paulo. Mobilidade acessível na cidade de São Paulo. Acessibilidade. Edificações, vias públicas, leis e normas. São Paulo, 2005.

Souza JJG. Hipertensão arterial e condições de vida: desigualdade em saúde entre pessoas que referiram hipertensão arterial em inquérito domiciliar de base populacional na Região Metropolitana de São Paulo. São Paulo; 2005. [Tese de Mestrado - Faculdade de Saúde Pública da Universidade de São Paulo].

Steinberg AG; Sullivan VJ; Loew R. Cultural and linguistic barriers to mental health services access: the deaf consumer's perspective. Am J Psychiatric., 1998; 155(7): 982-4.

Steinberg AG; Barnett S; Meador HE; Wiggins EA; Zazove P. Health care system accessibility. Experiences and perceptions of deaf people. J Gen Intern Med., 2006; 21(3): 260-6.

Sutton JP; Dejong G. Managed care and people with disabilities: framing the issues. Arch Phys Med Rehabil., 1998; 79(10): 1312-6.

Talbot LR; Viscogliosi C; Desrosiers J; Vincent C; Rosseau J; Robichaud L. Identification of rehabilitation needs after a stroke: an exploratory study. Health and Quality of Life Outcomes, 2004; 2: 53-61.

Thapar N; Warner G; Drainoni ML; Williams SR; Ditchfield H; Wierbick J; Nesathurai S. A pilot study of functional access to public buildings and facilities for persons with impairments. Disabil Rehabil, 2004; 26(5): 280-9.

Turrini RNT; Lebrão ML; Cesar CLG. Resolutividade dos serviços de saúde por inquérito domiciliar: percepção do usuário. Cadernos de Saúde Pública 2008; 24 : 663-674.

Ubido J; Huntington J; Warburnt D. Inequalities in access to healthcare faced by women who are deaf. Health and Social Care in the Community, 2002; 10(4): 24753.

Veltman A; Stewart DE; Tardif GS; Branigan M. Perceptions of primary healthcare services among people with physical disabilities. Part 1: Access issues. Medscape Gen Med., 2001;3(2). 
Wallace SP; Levy-Storms L; Ferguson LR. Access to paid in-home assistance among disabled elderly people: do Latinos differ from non-Latino whites? American Journal of Public Health, 1995; 85(7): 970-5.

Werngren-Elgström M, Carlsson G, Iwarsson S. A 10-year follow-up study on subjective well-being and relationships to person-environment (P-E) fit and activity of daily living (ADL) dependence of older Swedish adults. Arch Gerontol Geriatr. 2009; 49(1):e16-22.

Wharton S; Hames A; Milnet H. The accessibility of general NHS services for children with disabilities. Child: Care, Health \& Development, 2005; 31(3): 275-82.

Whiteneck G; Dijkers MP. Difficult to measure constructs: conceptual and methodological issue concerning participating and environment factors. Arch Phys Med Rehabil, 2009; 90 (Supp.1): S22-S35.

Willians MH; Bowie C. Evidence of unmet need in the care of severely physically disabled adults. British Medical Journal, 1993; 306(6870): 95-9.

Wilson K; Rosenberg MW. Accessibility and the Canadian health care system: squaring perceptions and realities. Health Policy, 2004; 67(2): 137-48.

Witt WP; Kasper JD; Riley AW. Mental health services use among school-aged children with disabilities: the role of socio-demographics, functional limitations, family burdens, and care coordination. Health Services Research, 2003; 38(6): 144166.

Zaitune MPA; Barros MBA; Cesar CLG. Hipertensão arterial em idosos:

prevalência, fatores associados e práticas de controle no Município de Campinas, São Paulo, Brasil. Cad de Saú Púb, 2006, 22(2): 285-294. 


\section{ANEXOS}

\subsection{ANEXO I - QUESTIONÁRIO ABERTO UTILIZADO NA FASE I DO PROJETO ACESS}

\section{Roteiro para Entrevista}

1.

a. Quando o(a) senhor(a) tem algum problema de saúde, o senhor(a) procura o serviço público ou o privado? Por quê?

b. Que tipo de serviço o senhor procura? Por quê?

2. Como o(a) senhor(a) faz para ir ate o serviço de saúde?.......Fale um pouco mais sobre isso.

3. Muitos serviços de saúde oferecem dificuldades para os deficientes como falta de rampas, estacionamentos, sinalização, etc. E para o(a) senhor(a) como tem sido isso?

4. De acordo com sua experiência, você considera o atendimento dos serviços de saúde que o(a) senhor(a) já recebeu adequado para um paciente com deficiência? Por quê?

5. Alguns deficientes se queixam que as informações recebidas por eles de médicos, enfermeiros e funcionários não são as mais adequadas. Como tem sido isso para você?

6. Vamos falar agora sobre exames de saúde. Pensando como deficiente, eu gostaria que me contasse como tem sido para você realizar exames de sangue, raios-X, papanicolau, próstata, urina, mamografia, diabetes, etc. 
7. Agora vamos falar de equipamentos para deficientes (muletas, cadeira de rodas, óculos, aparelhos de surdez, próteses, bolsas coletoras, etc). No seu caso, como tem sido conseguir esses equipamentos?...Fale um pouco mais sobre isso.

\section{Agora só faltam mais 2 perguntinhas.....}

8. No seu atendimento em saúde o(a) senhor(a) tem participado de alguma atividade educativa, orientação, campanha de prevenção, etc?... Fale um pouco mais sobre isso.

9. $\mathrm{O}$ (a) senhor(a) tem plano de saúde privado?
a. $\mathrm{O}$ (a) senhor(a) está satisfeito com ele?
b. Porque o(a) senhor(a) resolveu adquirir um plano de saúde? 


\subsection{ANEXO II - QUESTIONÁRIO FECHADO UTILIZADO NA FASE II DO PROJETO ACESS}

Faculdade de Saúde Pública - Universidade de São Paulo

\section{CONTROLE}

\section{BLOCO A}

Etiqueta com:

A 01. Número do questionário

A 02. Setor

A 03. Área

A 04. Nome do entrevistado

A 05. Data de Nascimento Idade

A 06. Sexo

A 07. Tipo de deficiência Endereço

Endereço atualizado:

Tel.:

Cel.:

Recados:

\begin{tabular}{|c|c|c|c|c|c|c|}
\hline visita & data & hora & $\begin{array}{l}\text { nome do } \\
\text { entrevistador }\end{array}$ & observações: & $\begin{array}{l}\text { resultado } \\
\text { da visita }\end{array}$ & $\begin{array}{l}\text { 1. realizada } \\
\text { 2. adiada } \\
\text { 3. morador ausente }\end{array}$ \\
\hline 1 & I & & & & & 4. recusa total \\
\hline 2 & I & & & & & $\begin{array}{l}\text { 5. recusa parcial } \\
\text { 6. falecido }\end{array}$ \\
\hline 3 & l & & & & & 7. morador desconhecido \\
\hline 4 & I & & & & & $\begin{array}{l}\text { 8. impossibilitado de } \\
\text { responder }\end{array}$ \\
\hline 5 & I & & & & & 9. outros, especif.: \\
\hline
\end{tabular}

A 10. Resultado das visitas:

A 11. $\mathbf{N}^{0}$. de visitas:

A 12. Entrevistador:

A 13. Data da entrevista: $-/ 2008$

Observações: 
A 20. Quem respondeu o questionário?

passe p/ questão A 25.

당

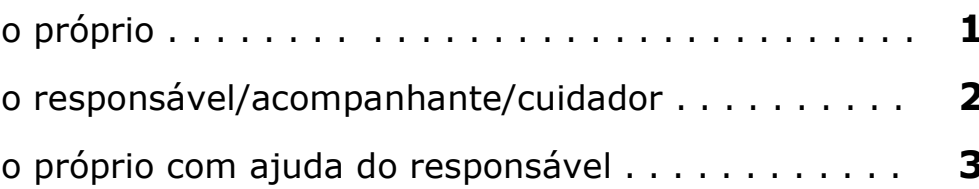

A 20

A 21. Nome do responsável/acompanhante/cuidador:

A 22. $O(a) \operatorname{sr}(a)$. é o que do(a)

pai $\ldots \ldots \ldots \ldots \ldots \ldots \ldots \ldots \ldots \ldots \ldots \ldots \ldots$ o1

mãe $\ldots \ldots \ldots \ldots \ldots \ldots \ldots \ldots \ldots \ldots \ldots \ldots \ldots$

$\operatorname{irmã(0)\ldots \ldots \ldots \ldots \ldots \ldots \ldots }$

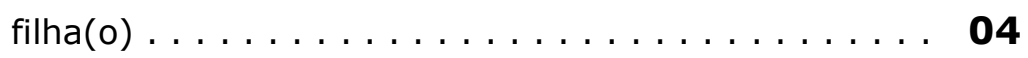

nora(genro) $\ldots \ldots \ldots \ldots \ldots \ldots \ldots$

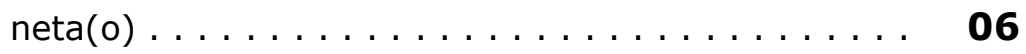

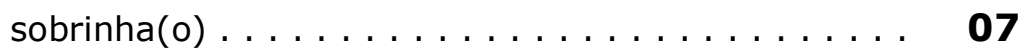

$\operatorname{prima}(0) \ldots \ldots \ldots \ldots \ldots \ldots \ldots \ldots$

$\operatorname{amiga}(0) \ldots \ldots \ldots \ldots \ldots \ldots \ldots$

contratada(o) da família . . . . . . . . . . . 10

outros, especif.: 11

A 23. Quantos dias por semana o(a) $\operatorname{sr}(a)$. passa cuidando do(a) $\operatorname{sr}(a)$.

A 24. Em que períodos o(a) $\operatorname{sr}(a)$. fica com o(a) $\operatorname{sr}(a)$.

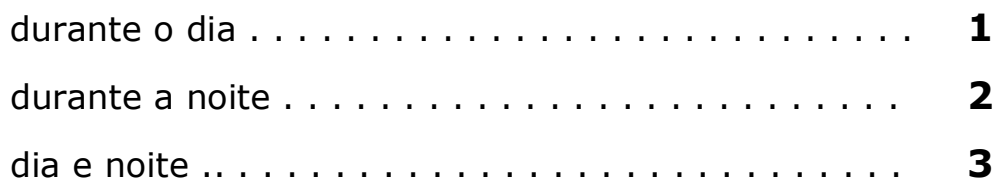

A 25. Na pesquisa anterior (2001/2003) o(a) $\operatorname{sr}(a)$. relatou A 25 ter (deficiência relatada). $\mathbf{O}(\mathbf{a}) \mathbf{s r}(\mathbf{a})$. ainda tem essa dificuldade?

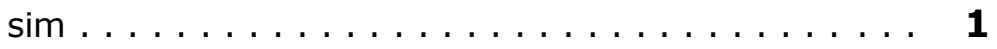

passe $p$ / questão A 28. não $\ldots \ldots \ldots \ldots \ldots \ldots \ldots \ldots \ldots$

passe $p /$ questão A $28 . \quad \mathrm{NS} / \mathrm{NR} \ldots \ldots \ldots \ldots \ldots \ldots \ldots \ldots$

A 26. O que aconteceu com essa deficiência de 2001/2003 até hoje? ( leia as alternativas para o entrevistado) passe $p /$ questão A 28 . não mudou nada $\ldots \ldots \ldots \ldots \ldots \ldots \ldots \ldots$ 
se agravou ................. 2

melhorou .................. 3

passe $p /$ questão A $28 . \quad \mathrm{NS} / \mathrm{NR} \ldots \ldots \ldots \ldots \ldots \ldots \ldots \ldots$

A 27. Como isso aconteceu?

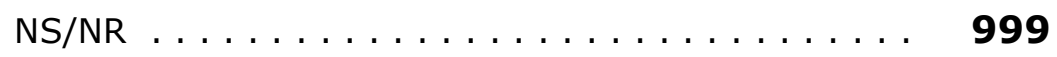

\section{A 28. ALÉM do problema citado na entrevista anterior, hoje o(a) sr(a). tem algum} desses problemas?

Pode haver mais de uma resposta (leia as alternativas para o entrevistado)

cegueira de um olho . . . . . . . . . . . 03

cegueira de dois olhos .............. 04

dificuldade de ouvir ............... 05

surdez de um ouvido .............. 06

surdez de dois ouvidos . . . . . . . . . . . . 07

paralisia total ou parcial de membros.
especif.:
perda de membros ou parte deles.
especif.:
outro, especif.:
99

A 29. O(a) sr(a). precisa de ajuda de alguém para realizar alguma das seguintes

A 29 atividades?

Pode haver mais de uma resposta (leia as alternativas para o entrevistado)

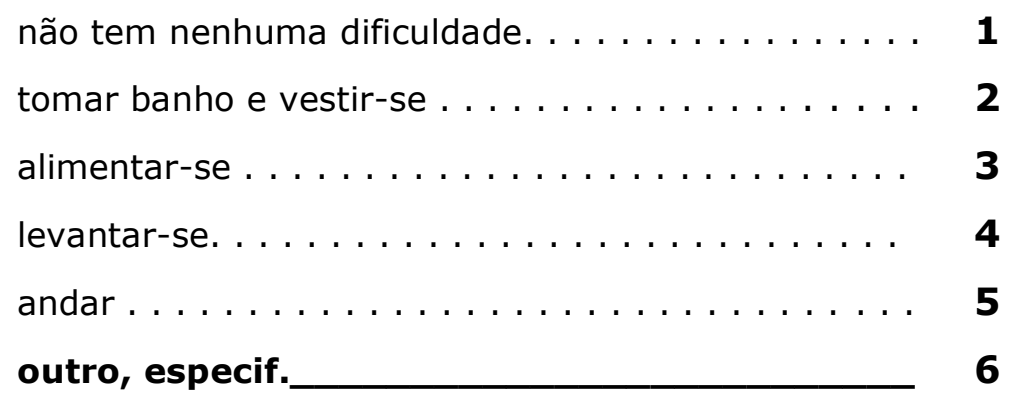


Deficiência referida pelo entrevistado:

\begin{tabular}{|l|l|}
\hline dificuldade de enxergar, mesmo com óculos/ lentes & \\
\cline { 1 - 1 } cegueira de um olho & \multirow{2}{*}{ responda } \\
\cline { 1 - 1 } cegueira de dois olhos & \\
\cline { 1 - 1 } dificuldade de ouvir & \\
\cline { 1 - 1 } surdez de um ouvido & \multirow{2}{*}{ responda } \\
\cline { 1 - 1 } surdez de dois ouvidos & BLOCO B2 \\
\cline { 1 - 1 } paralisia total ou parcial de membros & \\
\cline { 1 - 2 } perda de membros ou parte deles &
\end{tabular}


B1-01. Quando foi a última vez que o sr.(a) procurou um serviço de saúde? (hospital, posto de saúde, ambulatório, AMA, dentista, clínica)

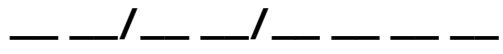

NS/NR

$99 / 99 / 99$

B1-02. Qual foi o último serviço de saúde que o(a) sr.(a) procurou? (considerar o último serviço de saúde procurado)

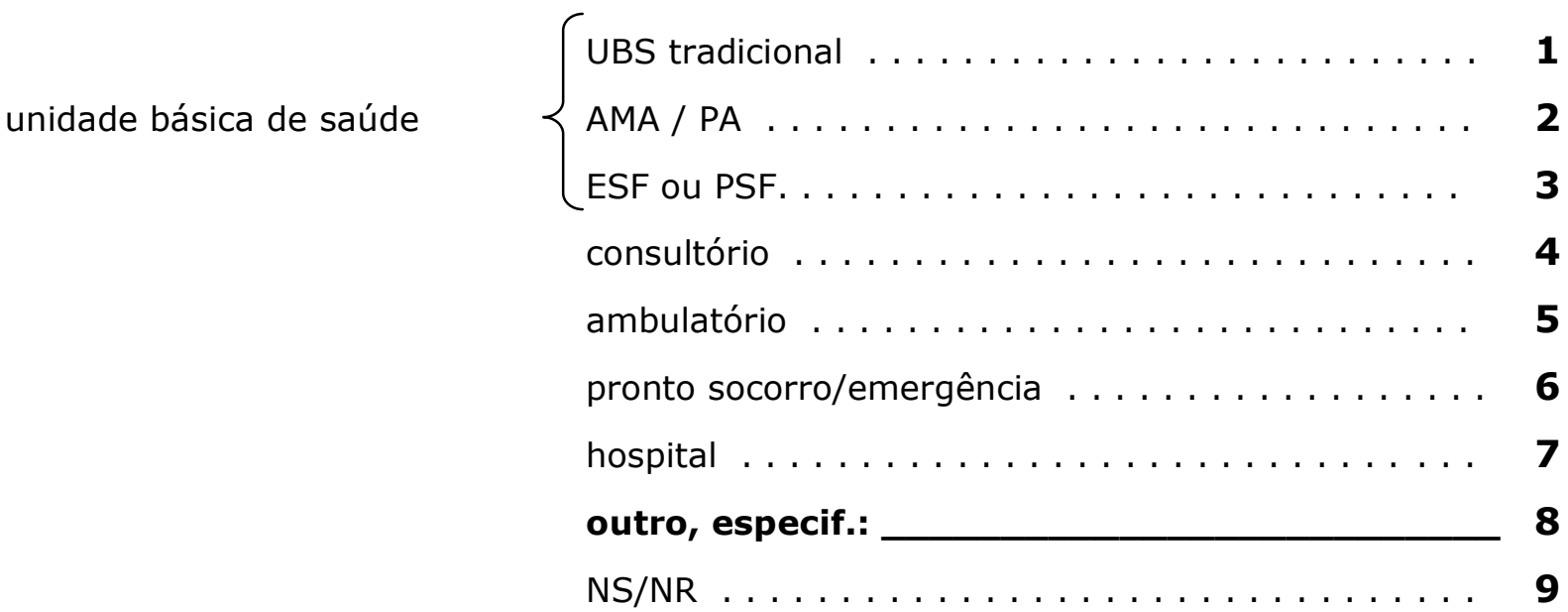

B1-03. Esse serviço de saúde é: (leia as alternativas para o entrevistado)

sus $\ldots \ldots \ldots \ldots \ldots \ldots \ldots \ldots \ldots \ldots$

previdência governamental (Fed./Est./Mun.) . . . . . 2

privado/particular ............... 3

serviço de sindicato/ associação de categoria . . . . . 4

serviço próprio de empresa . . . . . . . . . . 5

outro, especif.: _ 6

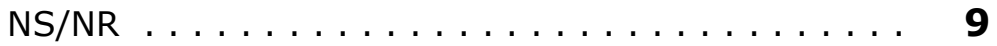


B1-04. Qual(is) foi(ram) o(s) motivos que o levaram a procurar esse serviço? Pode haver mais de uma resposta

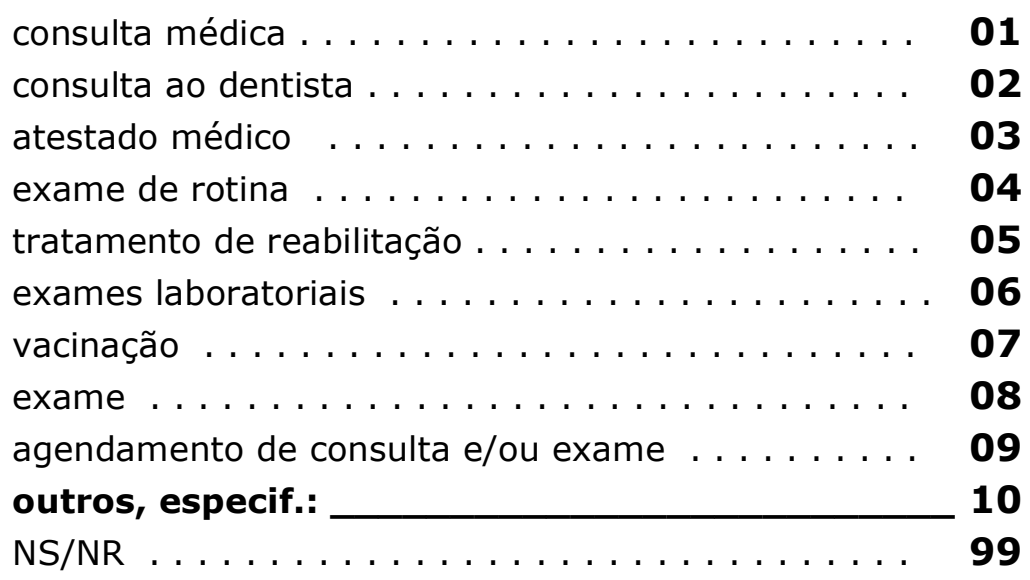

B1-05. Quem cobriu ou complementou os gastos?

Pode haver mais de uma resposta

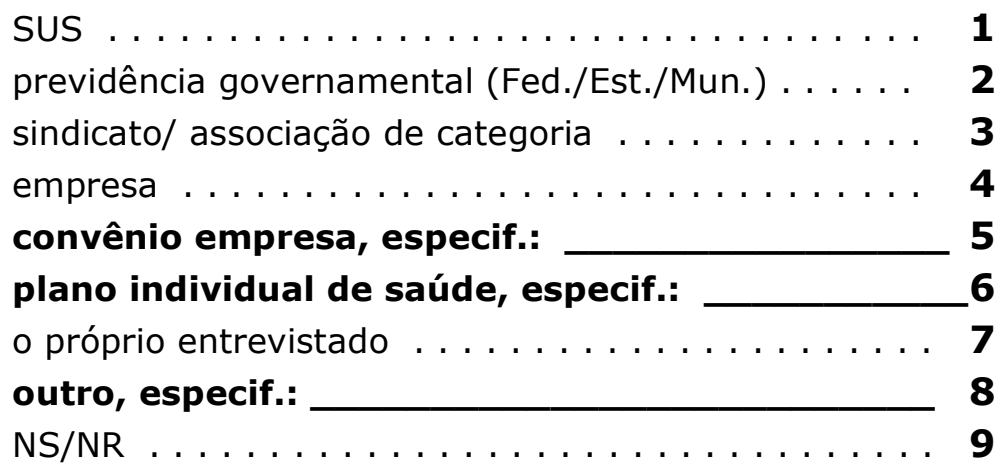

B1-06. Na última vez que o(a) sr(a). foi a um serviço de saúde precisou de um acompanhante em decorrência de sua deficiência?

passe $p /$ questão $\mathbf{B 1 - 0 8 . ~}$

passe p/ questão B1-08.

B1-07. Por quê?
1 2

9

NS/NR 
B1-08. Na última vez que o(a) sr(a). foi a um serviço de saúde, como se deslocou até lá? Pode haver mais de uma resposta

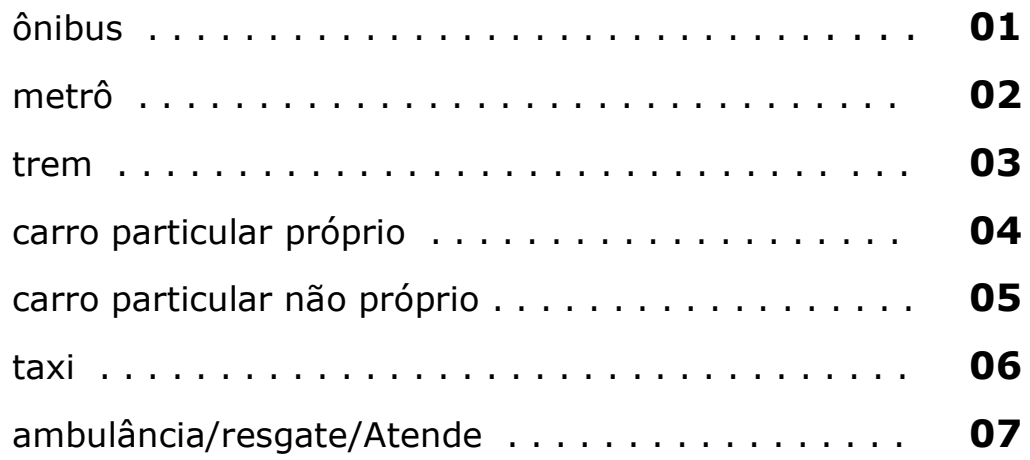

passe $p /$ questão B1-12.

outro, especif.:

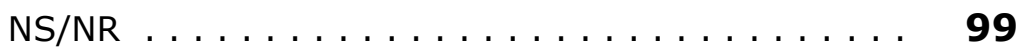

B1-09. O(a) sr(a). em decorrência de sua deficiência, teve dificuldade no uso desse transporte? (falta de espaço para cadeira de rodas, falta de sinalização, poltronas reservadas, necessidade de um carro adaptado, etc...)

passe $p /$ questão B1-11.

$\operatorname{sim} \ldots \ldots \ldots \ldots \ldots \ldots \ldots$

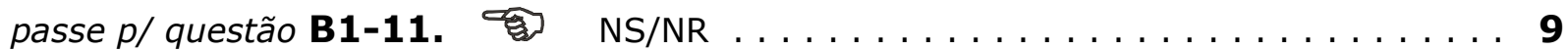

B1-10. Qual(is) dificuldades teve?

B1-11. Existe algum outro problema no transporte que o(a) $\operatorname{sr}(a)$ usa para se deslocar aos serviços de saúde que queira relatar?

Sim, espcifi.

não

NS/NR 
B1-12. No último serviço de saúde que o(a) sr.(a) que utilizou, teve problemas com: Pode haver mais de uma resposta ( leia as alternativas para o entrevistado) estacionamento com vaga para pessoas com deficiência . . . $\mathbf{1}$ p/ deficientes auditivos sinalização para localização e deslocamento interno e externo $\mathbf{2}$

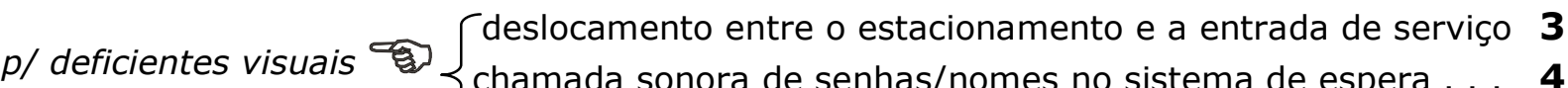
chamada sonora de senhas/nomes no sistema de espera . . . 4 deslocamento interno (corredores,salas,etc.) . . . . . . 5 não teve nenhum problema . . . . . . . . . . . . 6

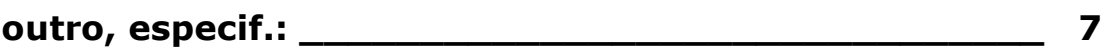

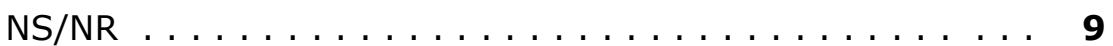

B1-13. O(a) sr.(a) tem ou já teve convênio ou plano de saúde médico ou odontológico?

já teve, não tem mais $\ldots \ldots \ldots \ldots \ldots \ldots \ldots$

encerre e leia a chamada no final do bloco

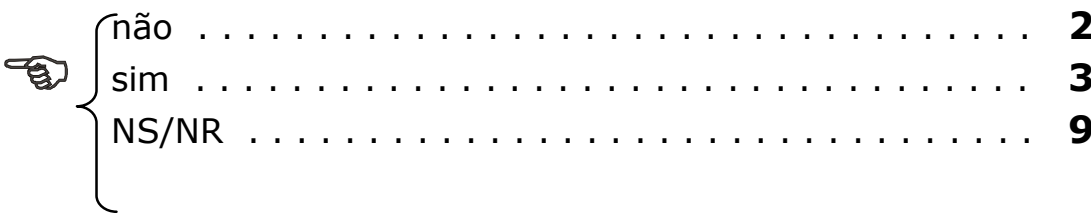

B1-14. Por que o(a) sr.(a) não tem mais o convênio ou plano de saúde?

ficou muito caro e não conseguiu pagar . . . . . . $\mathbf{1}$

era convênio empresa e não trabalha mais lá . . . . . 2 perdeu quando ficou doente ou com deficiência . . . . 3

outro, especif.: _ 4

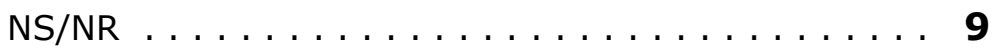

Se o entrevistado for também deficient
físico responda o BLOCO B2, começand
pela questão B2-09.

CASO CONTRÁRIO:

Mulheres com 20 anos ou mais? Homens com 40 anos ou mais responda o BLOCO C e siga p/BLOCO D.

Mulheres menores de 20 anos\} Homens menores de 40 anos $\}$ 
Deficientes físicos

Usos de Serviços de Saúde - Acessibilidade

BLOCO B2

B2-01. Quando foi a última vez que o(a) sr.(a) procurou um serviço de saúde?

(hospital, posto de saúde, ambulatório, AMA, dentista, clínica)

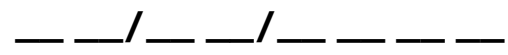

NS/NR

$99 / 99 / 99$

B2-02. Qual foi o último serviço de saúde que o(a) sr.(a) procurou?

(considerar o último serviço de saúde procurado)

unidade básica de saúde

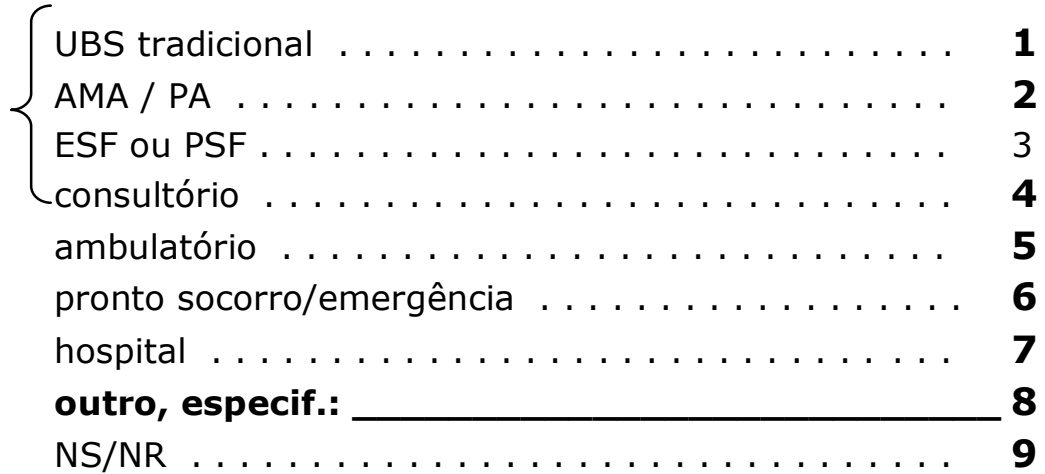

B2-03. Esse serviço de saúde é: (leia as alternativas para o entrevistado)

SUS .................. 1

previdência governamental (Fed./Est./Mun.) . . . . 2

privado/particular ............... 3

serviço de sindicato/ associação de categoria . . . . . 4

serviço próprio de empresa . . . . . . . . . . 5

outro, especif.:

NS/NR 
B2-04. Qual(is) foi(ram) o(s) motivos que o levaram a procurar esse serviço? Pode haver mais de uma resposta

consulta médica . . . . . . . . . . . . 01

consulta ao dentista . . . . . . . . . . . . 02

atestado médico $\ldots \ldots \ldots \ldots \ldots \ldots \ldots$

exame de rotina . . . . . . . . . . . . . . 04

tratamento de reabilitação . . . . . . . . . . 05

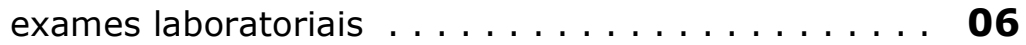

vacinação .................... 07

exame .................. 08

agendamento de consulta e/ou exame ........ 09

outros, especif.: _ 10

NS/NR $\ldots \ldots \ldots \ldots \ldots \ldots \ldots \ldots \ldots \ldots \ldots$

B2-05. Quem cobriu ou complementou os gastos?

Pode haver mais de uma resposta

sus $\ldots \ldots \ldots \ldots \ldots \ldots \ldots \ldots \ldots$

previdência governamental (Fed./Est./Mun.) . . . . 2

sindicato/ associação de categoria . . . . . . . 3

empresa ................... 4

convênio empresa, especif.:

plano individual de saúde, especif.: _ 6

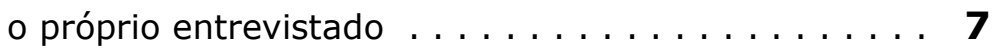

outro, especif.: _ 8

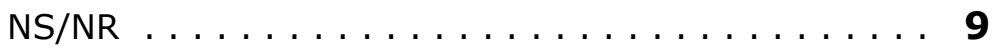

B2-06. Na última vez que o(a) sr(a). foi a um serviço de saúde precisou de um acompanhante em decorrência de sua deficiência?

passe $p$ / questão B2-08.

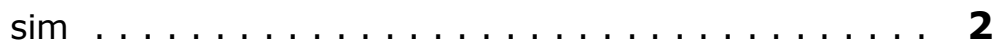

passe $p$ / questão B2-08. $\quad$ NS/NR $\ldots \ldots \ldots \ldots \ldots \ldots \ldots \ldots \ldots$

B2-07. Por quê? 
B2-08. Na última vez que o(a) sr(a). foi a um serviço de saúde, como se deslocou até lá? Pode haver mais de uma resposta

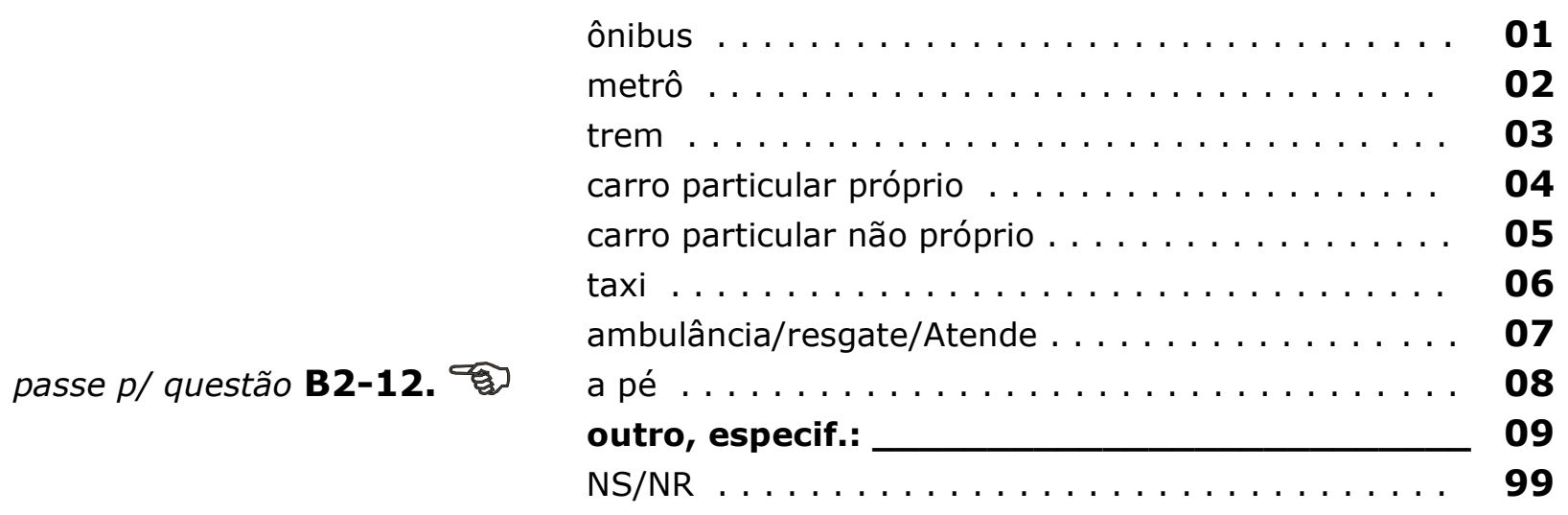

B2-09. O(a) sr(a). em decorrência de sua deficiência, teve dificuldade no uso desse transporte? (falta de espaço para cadeira de rodas, falta de sinalização, poltronas reservadas, necessidade de um carro adaptado, etc...)

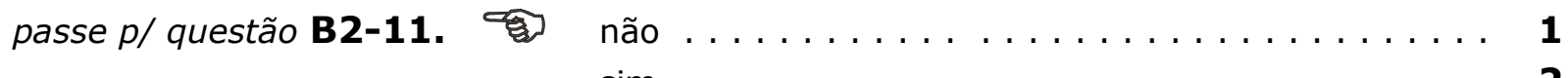

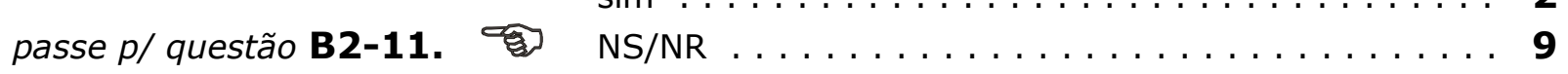

B2-10. Qual(is) dificuldade(s) teve?

B2-11. Existe algum outro problema no transporte que o(a) $\operatorname{sr}(a)$ usa para se deslocar aos serviços de saúde que queira relatar?

Sim, especifi.

não $\ldots \ldots \ldots \ldots \ldots \ldots \ldots \ldots \ldots \ldots \ldots \ldots \ldots \ldots \ldots$

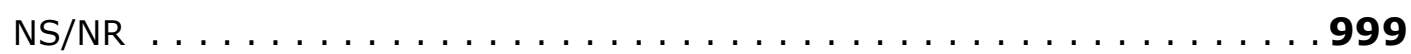


B2-12. No último serviço de saúde que o(a) sr.(a) que utilizou, teve problemas com vagas de estacionamento para pessoas com deficiência?

passe $p /$ questão B2-14. 㩆 não $\ldots \ldots \ldots \ldots \ldots \ldots \ldots \ldots \ldots$

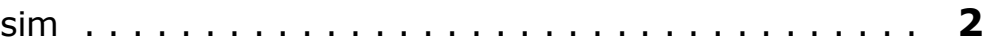

B2-12

passe $p /$ questão B2-16. não se aplica, não usou estacionamento. . . . . . . . 3

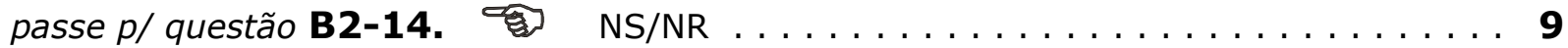

B2-13. Esse problema prejudicou o uso do serviço de saúde?

não prejudicou $\ldots \ldots \ldots \ldots \ldots \ldots \ldots \ldots$

prejudicou parcialmente . . . . . . . . . . $\mathbf{2}$

prejudicou totalmente .............. $\mathbf{3}$

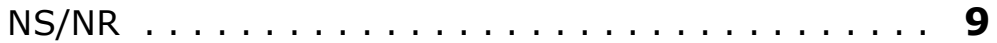

B2-14. No último serviço de saúde que o(a) sr.(a) que utilizou, teve problemas com deslocamento entre o estacionamento e a entrada do serviço?

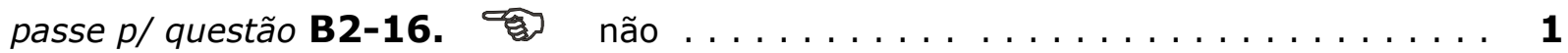

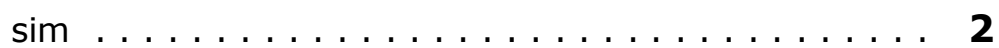

passe $p /$ questão B2-16. $\quad$ NS/NR $\ldots \ldots \ldots \ldots \ldots \ldots \ldots \ldots$

B2-15. Esse problema prejudicou o uso do serviço de saúde?

não prejudicou $\ldots \ldots \ldots \ldots \ldots \ldots \ldots \ldots$

prejudicou parcialmente .............. $\mathbf{2}$

prejudicou totalmente ............. 3

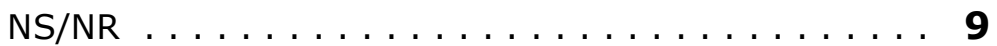

B2-16. No último serviço de saúde que o(a) sr.(a) que utilizou, teve problemas com rampas ou escadas?

passe $p$ / questão B2-18. não $\ldots \ldots \ldots \ldots \ldots \ldots \ldots \ldots \ldots \ldots \ldots$

B2-16

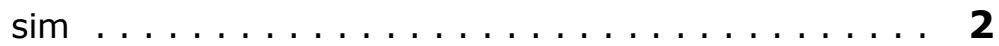

passe $p /$ questão B2-18. $\quad \mathrm{NS} / \mathrm{NR} \ldots \ldots \ldots \ldots \ldots \ldots$ 
B2-17. Esse problema prejudicou o uso do serviço de saúde?

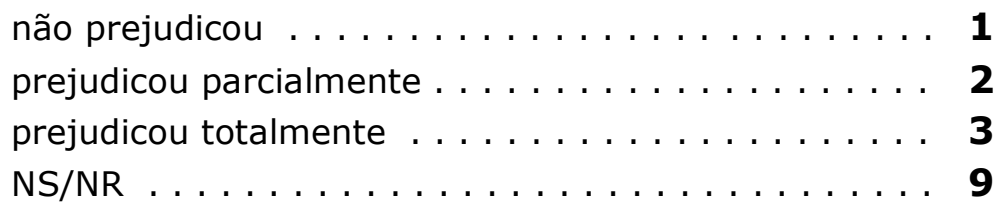

B2-18. No último serviço de saúde que o(a) sr.(a) que utilizou, teve problemas com corrimãos em rampas e escadas?

passe $p$ / questão B2-20. não $\ldots \ldots \ldots \ldots \ldots \ldots \ldots \ldots \ldots \ldots$

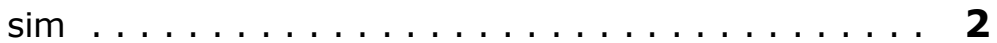

passe $p$ / questão B2-20. $\mathrm{NS/NR} \ldots \ldots \ldots \ldots \ldots \ldots \ldots \ldots \ldots$

B2-19. Esse problema prejudicou o uso do serviço de saúde?

não prejudicou $\ldots \ldots \ldots \ldots \ldots \ldots \ldots \ldots \ldots$

prejudicou parcialmente . . . . . . . . . . $\mathbf{2}$

prejudicou totalmente .............. 3

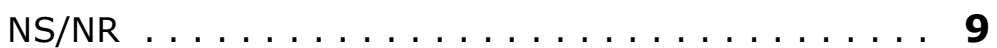

B2-20. No último serviço de saúde que o(a) sr.(a) que utilizou, teve problemas com elevadores?

passe $p$ / questão B2-22. 数 não $\ldots \ldots \ldots \ldots \ldots \ldots \ldots \ldots \ldots \ldots$

$\operatorname{sim} \ldots \ldots \ldots \ldots \ldots \ldots \ldots \ldots \ldots \ldots \ldots$

passe $p /$ questão B2-22. $\quad$ NS/NR $\ldots \ldots \ldots \ldots \ldots \ldots \ldots \ldots \ldots$

B2-21. Esse problema prejudicou o uso do serviço de saúde?

não prejudicou $\ldots \ldots \ldots \ldots \ldots \ldots \ldots \ldots$

prejudicou parcialmente . . . . . . . . . . 2

prejudicou totalmente .............. 3

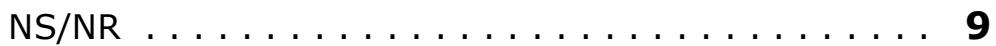

B2-22. No último serviço de saúde que o(a) sr.(a) que utilizou, teve problemas com cadeiras de rodas para transporte de pessoas com deficiência?

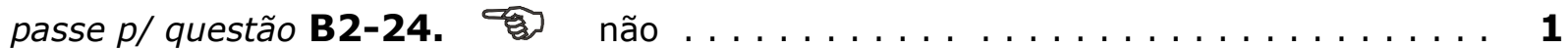

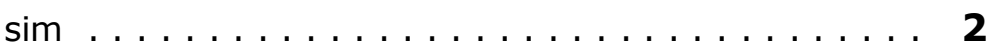

passe $p /$ questão B2-24. não se aplica, não usa cadeira de rodas. . . . . . . . 3

passe $p /$ questão B2-24. $\quad$ NS/NR $\ldots \ldots \ldots \ldots \ldots \ldots \ldots \ldots \ldots$ 
B2-23. Esse problema prejudicou o uso do serviço de saúde?

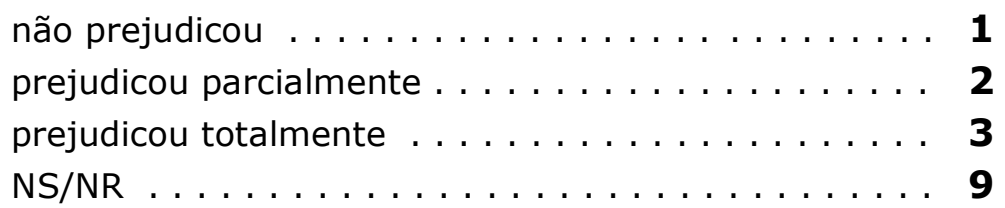

B2-24. No último serviço de saúde que o(a) sr.(a) que utilizou, teve problemas com sanitários adaptados ou reservados para pessoas com deficiência?

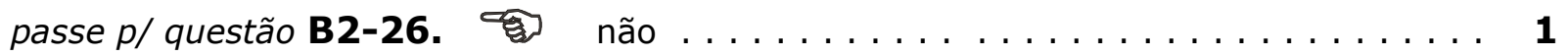

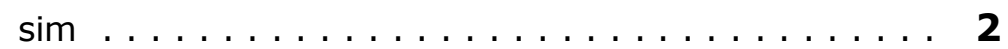

passe $p$ / questão B2-26. $\left\{\begin{array}{l}\text { não precisei utilizar. } \ldots \ldots \ldots \ldots \ldots \ldots \ldots \ldots \ldots \ldots \ldots \ldots \ldots \ldots \\ \text { não existe sanitário adaptado } \ldots \ldots \ldots \ldots \ldots \ldots \ldots \\ \text { NS/NR } \ldots \ldots \ldots \ldots \ldots \ldots\end{array}\right.$

B2-25. Esse problema prejudicou o uso do serviço de saúde?

não prejudicou $\ldots \ldots \ldots \ldots \ldots \ldots \ldots \ldots \ldots$

prejudicou parcialmente . . . . . . . . . . . $\mathbf{2}$

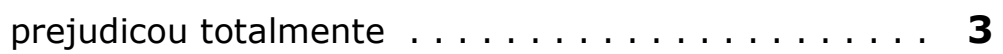

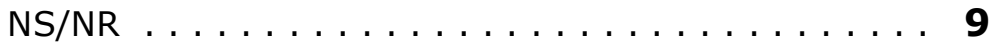

B2-26. No último serviço de saúde que o(a) sr.(a) que utilizou, teve problemas com o local/sala de espera?

passe $p$ / questão B2-28.

sim $\ldots \ldots \ldots \ldots \ldots \ldots \ldots \ldots \ldots \ldots \ldots \ldots \ldots, \mathbf{2}$

passe $p /$ questão B2-28. $\quad$ NS/NR $\ldots \ldots \ldots \ldots \ldots \ldots \ldots \ldots \ldots$

B2-27. Esse problema prejudicou o uso do serviço de saúde?

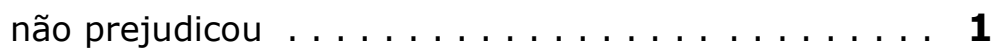

prejudicou parcialmente . . . . . . . . . . 2

prejudicou totalmente .............. 3

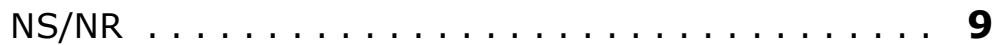

B2-28. No último serviço de saúde que o(a) sr.(a) que utilizou, teve problemas com a circulação interna, corredores, portas, salas, etc.?

passe p/ questão B2-30.

passe $p$ / questão B2-30.

할

não

$\operatorname{sim}$

NS/NR 
B2-29. Esse problema prejudicou o uso do serviço de saúde?

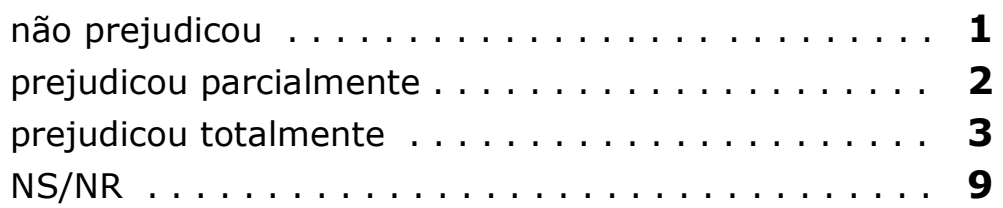

B2-30. No último serviço de saúde que o(a) sr.(a) que utilizou, teve algum outro problema?

passe $p$ / questão B2-32.

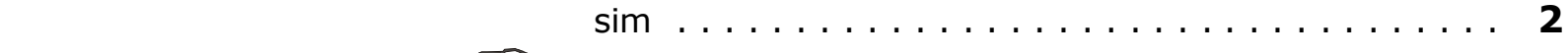

passe $p /$ questão B2-32. $\quad$ NS/NR $\ldots \ldots \ldots \ldots \ldots \ldots \ldots \ldots$

B2-31. Qual(is) problema(s) teve?

B2-32. O(a) sr.(a) tem convênio ou plano de saúde médico ou odontológico?

já teve, não tem mais $\ldots \ldots \ldots \ldots \ldots \ldots \ldots$

encerre e leia a chamada no final do bloco

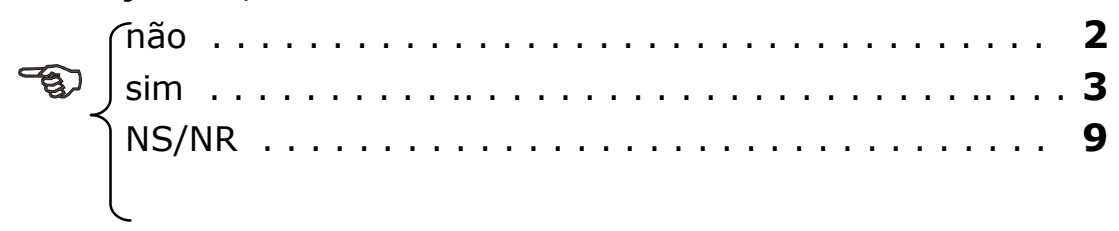

B2-33. Por que o(a) sr.(a) não tem mais o convênio ou plano de saúde?

ficou muito caro e não conseguiu pagar . . . . . . $\mathbf{1}$

era convênio empresa e não trabalha mais lá . . . . . 2

perdeu quando ficou doente ou com deficiência . . . . 3

outro, especif.: _ 4

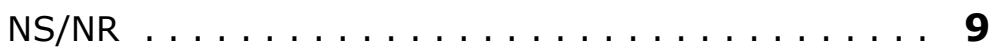

Mulheres com 20 anos ou mais? Homens com 40 anos ou mais 


\title{
Exames de Saúde - Orientações em Saúde
}

\author{
Agora vamos falar sobre exames de saúde
}

BLOCO C

\section{PARA TODAS AS MULHERES COM 20 ANOS OU MAIS}

C 01. O exame de Papanicolau é usado nos programas de prevenção de câncer de colo de útero. Quando foi que a sra. fez pela última vez este exame?

passe $p$ / questão $\mathbf{C} 06$.

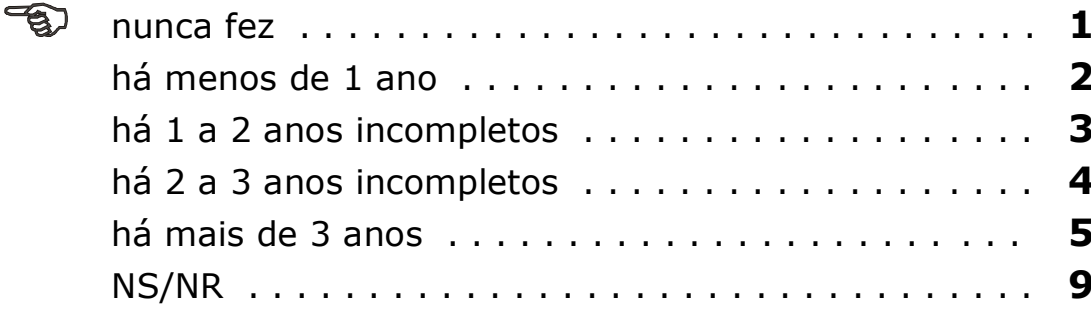

C 02. Em que serviço de saúde a sra. fez o exame?

unidade básica de saúde

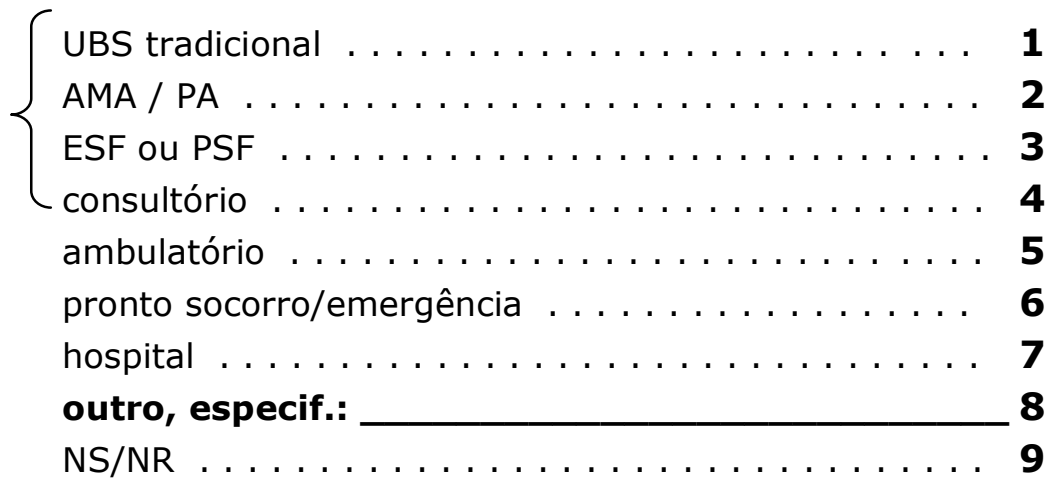


C 03. Quem cobriu ou complementou os gastos deste exame?

Pode haver mais de uma resposta

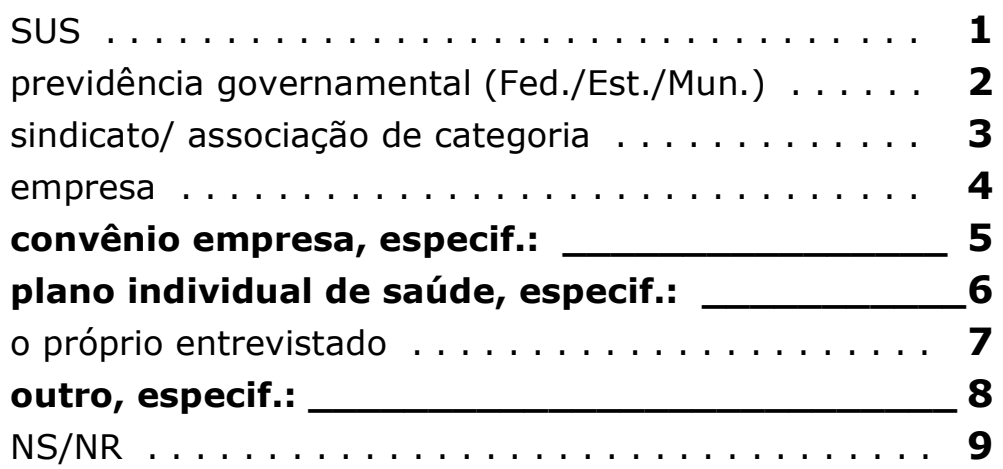

C 04. A sra. alguma vez teve problema(s) com a mesa ginecológica para realização do Papanicolau?

passe $p$ / questão $\mathbf{C} \mathbf{0 6 .}$ है:

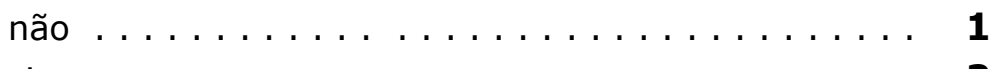

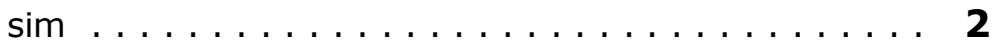

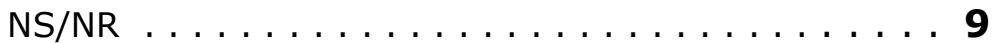

C 05. Que problema(s) teve?

NS/NR $\ldots \ldots \ldots \ldots \ldots \ldots \ldots \ldots \ldots \ldots$

C 06. O exame clínico das mamas é um exame feito por um médico ou enfermeiro para detectar a presença de nódulos/caroços nos seios. Quando foi que a sra. fez pela última vez este exame?

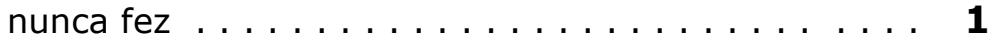

há menos de 1 ano ................ 2

há 1 a 2 anos incompletos ............. 3

há 2 a 3 anos incompletos ............. 4

há mais de 3 anos . . . . . . . . . . . . 5

NS/NR .................... 9 
C 07. A sra. faz palpação nos seus seios para procurar nódulos/ caroços? Com que freqüência?

Mulheres com:

40 anos ou + passe $p /$

C 12.

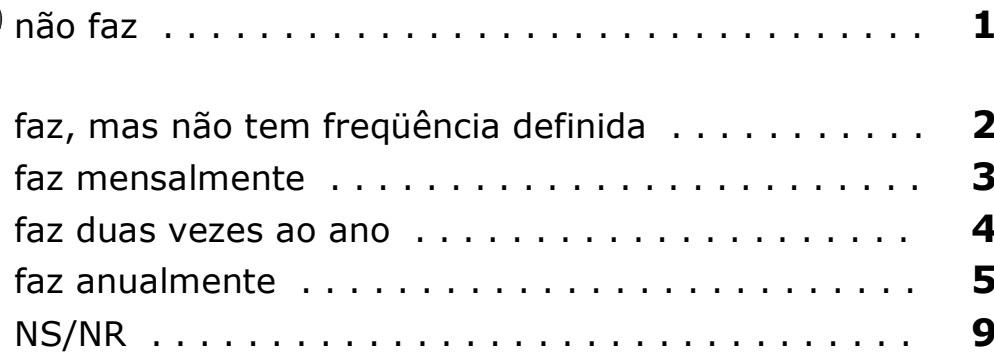

C 08. A sra. recebeu orientação de algum médico ou profissional de saúde sobre a necessidade de palpação das mamas?

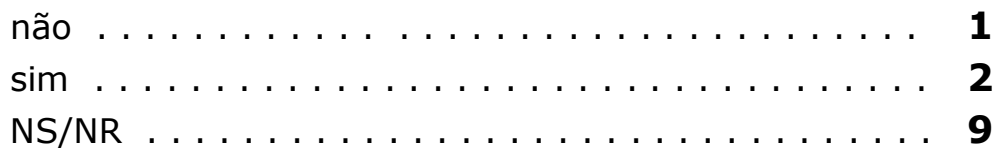

C 09. Quem ensinou a sra. a fazer o auto exame das mamas?

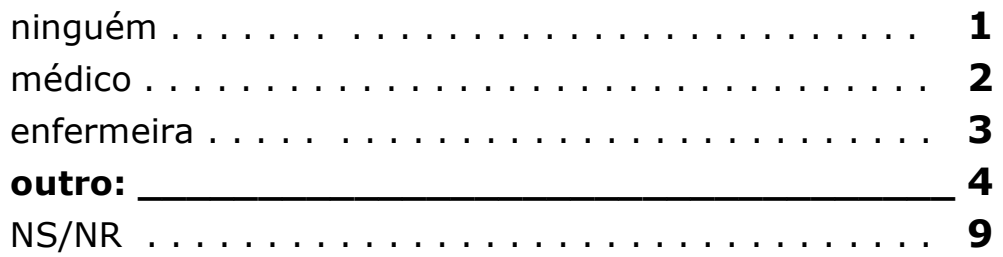

C 10. A sra. teve dificuldade para aprender a realizar o exame? Mulheres com:

40 anos ou + passe $p /$

- de 40 passe $p /$ Bloco D.

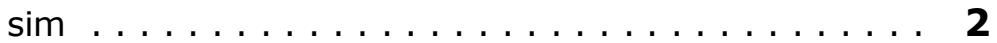

NS/NR

\section{PARA TODAS AS MULHERES COM 40 ANOS OU MAIS}

O Bloco se aplica a este entrevistado?

$\operatorname{sim} \ldots \ldots \ldots$

não . . . . . . 2

C 12. A mamografia é um raio $X$ dos seios, e é utilizada nos programas de prevenção 
de câncer de mama. Quando foi a última vez que a sra. fez este exame?

passe $p$ / questão $\mathbf{C 1 7}$.

है

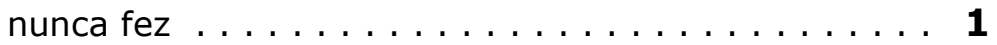

há menos de 1 ano ................ 2

há 1 a 2 anos incompletos ............ 3

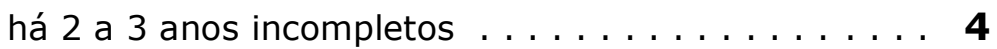

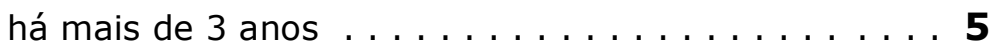

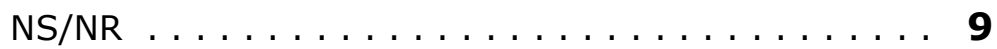

C 13. Em que serviço de saúde a sra. fez o exame?

unidade básica de saúde

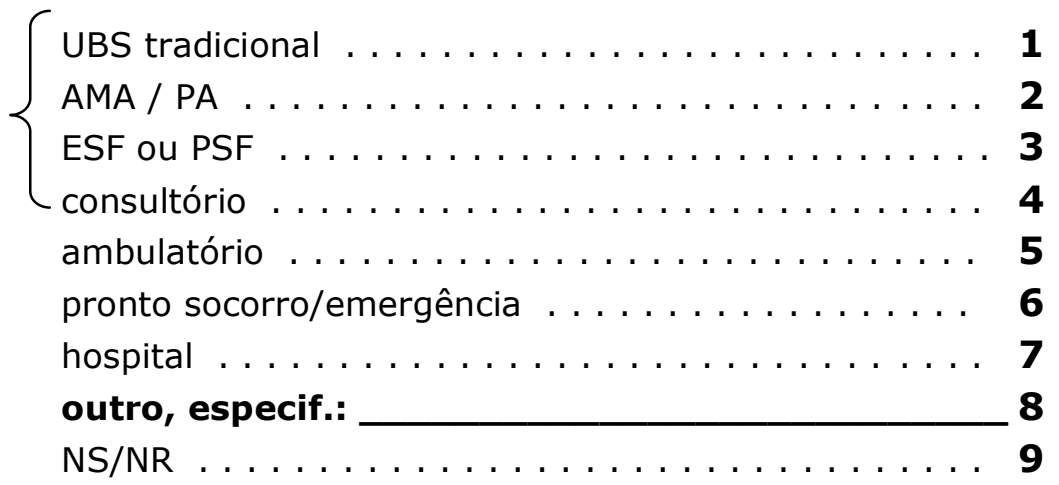

\section{14. Quem cobriu ou complementou os gastos deste exame?}

Pode haver mais de uma resposta

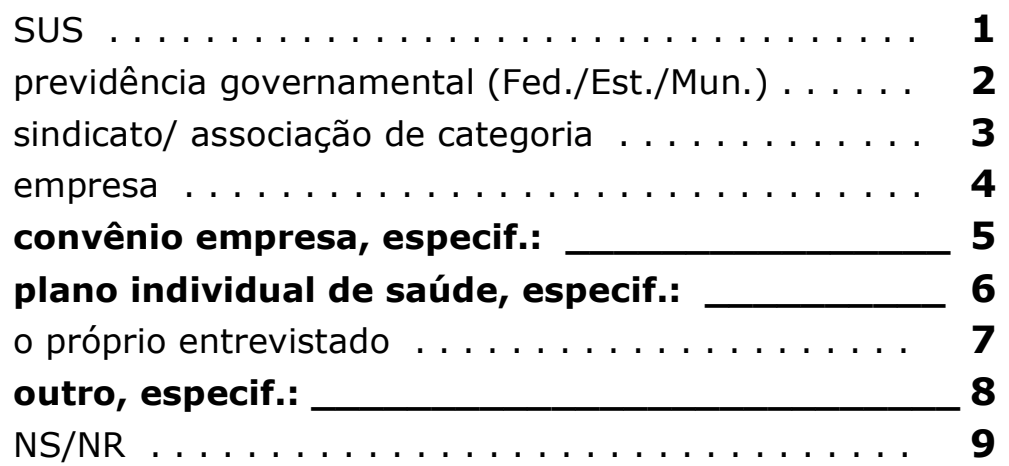


C 15. A sra. alguma vez teve problema com o equipamento para realização da mamografia?

passe $p /$ questão $\mathbf{C} \mathbf{1 7}$.

है:

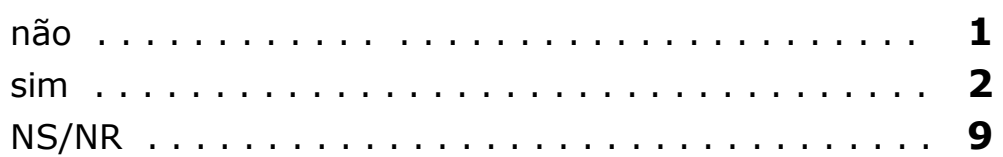

C 16. Que problema(s) teve?

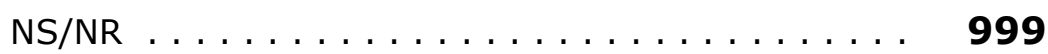

C 17. Existem exames utilizados nos programas de prevenção de câncer de intestino. A sra. já fez algum exame com esta finalidade? passe $p$ / o BLOCO D.

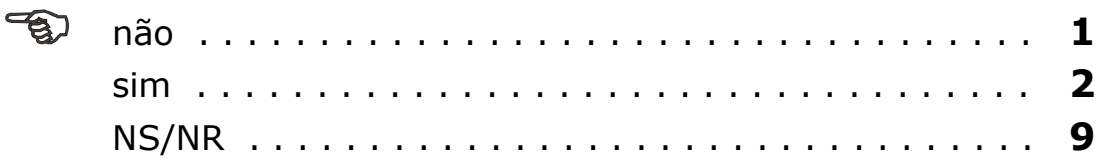

C 18. Qual(is) exame(s)?

Pode haver mais de uma resposta (leia as alternativas para o entrevistado)

fez pesquisa de sangue oculto nas fezes . . . . . . 1

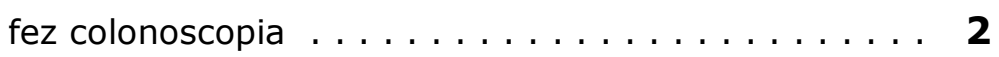

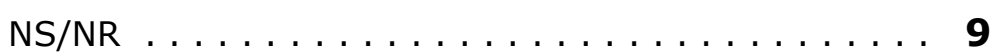

C 19. Quando foi a última vez que a sra. fez algum desses exames?

há menos de 1 ano . . . . . . . . . . . 1

há 1 a 2 anos incompletos . . . . . . . . . $\mathbf{2}$

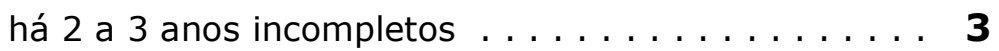

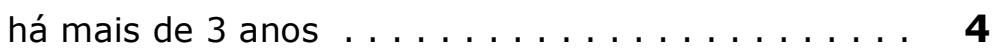

NS/NR ................ 9

C 20. Em que serviço de saúde a sra. fez o(s) exame(s)?

unidade básica de saúde

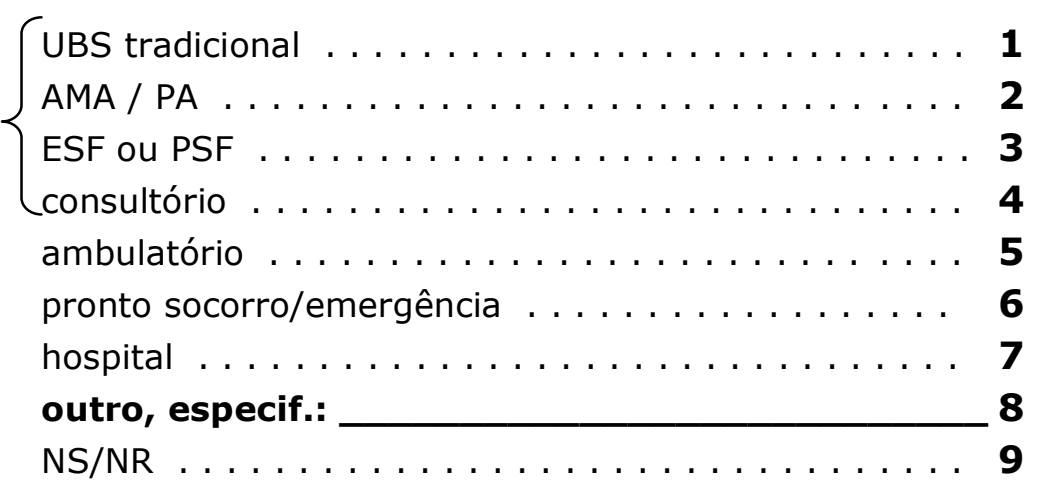


C 21. Esse serviço de saúde é: (leia as alternativas para o entrevistado)

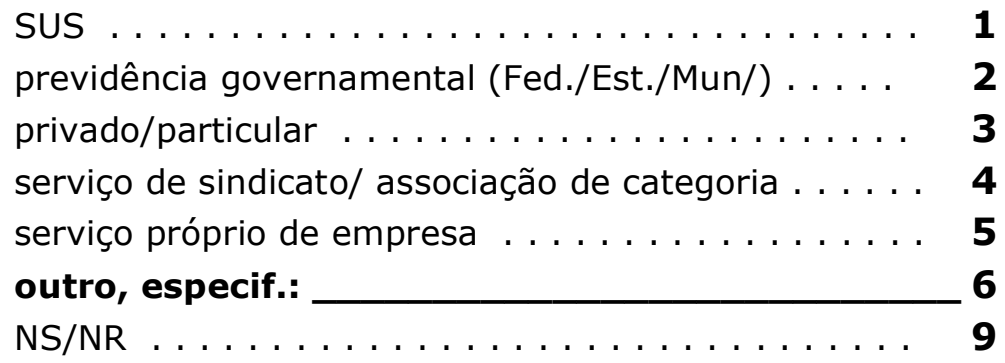

C 22. Quem cobriu ou complementou os gastos deste(s) exame(s)?

Pode haver mais de uma resposta

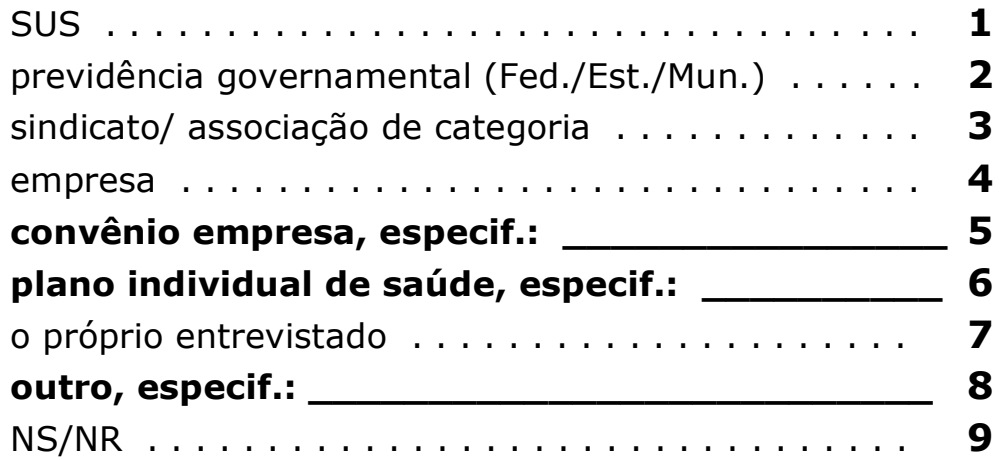

C 23. A sra. teve algum problema na realização desse(s) exame(s)?

passe $p /$ o BLOCO D. 잉

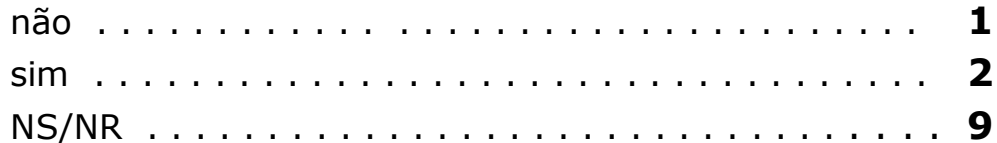

C 24. Que problema(s) teve? 


\section{S PARA TODOS OS HOMENS COM 40 ANOS OU MAIS}

C 25. Existem exames utilizados nos programas de prevenção de câncer de próstata. 0 sr. já fez algum exame com esta finalidade?

passe $p /$ questão $\mathbf{C} \mathbf{3 4 .}$

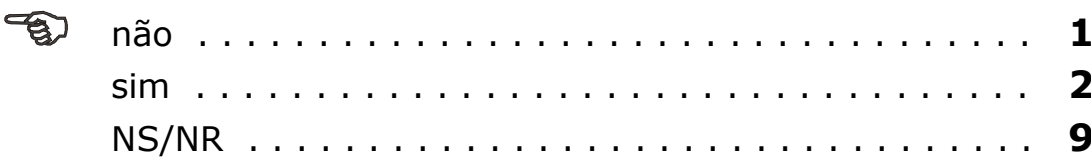

C 26. Qual(is) exame(s)?

Pode haver mais de uma resposta

( leia as alternativas para o entrevistado)

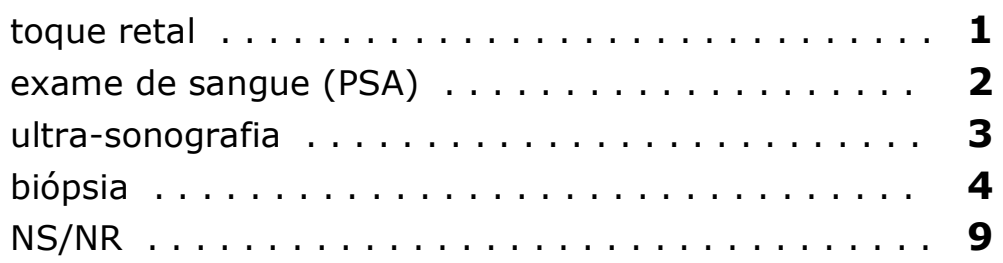

C 27. Quando foi a última vez que o sr. fez algum desses exames?

há menos de 1 ano . . . . . . . . . . . . $\mathbf{1}$

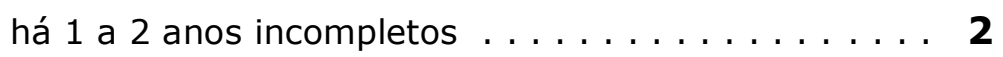

há 2 a 3 anos incompletos ............. 3

há mais de 3 anos ............... 4

NS/NR .................. 9

C 28. Em que serviço de saúde o sr. fez o(s) exame(s)?

unidade básica de saúde

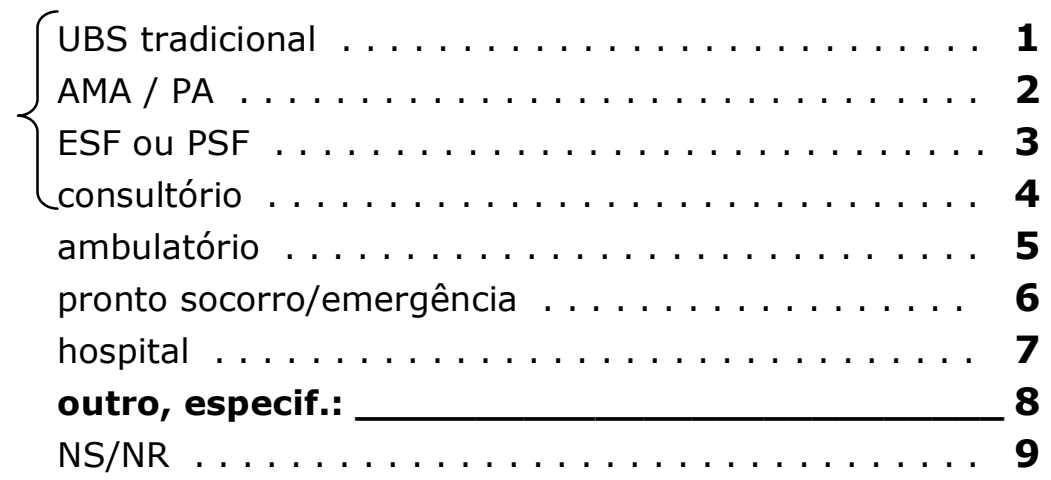


C 29. Esse serviço de saúde é: (leia as alternativas para o entrevistado)

sus ..................... 1

previdência governamental (Fed./Est./Mun.) . . . . 2

privado/particular ............... 3

serviço de sindicato/ associação de categoria . . . . . 4

serviço próprio de empresa . . . . . . . . . 5

outro, especif.: _ 6

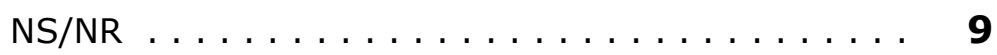

C 30. Quem cobriu ou complementou os gastos deste(s) exame(s)?

Pode haver mais de uma resposta

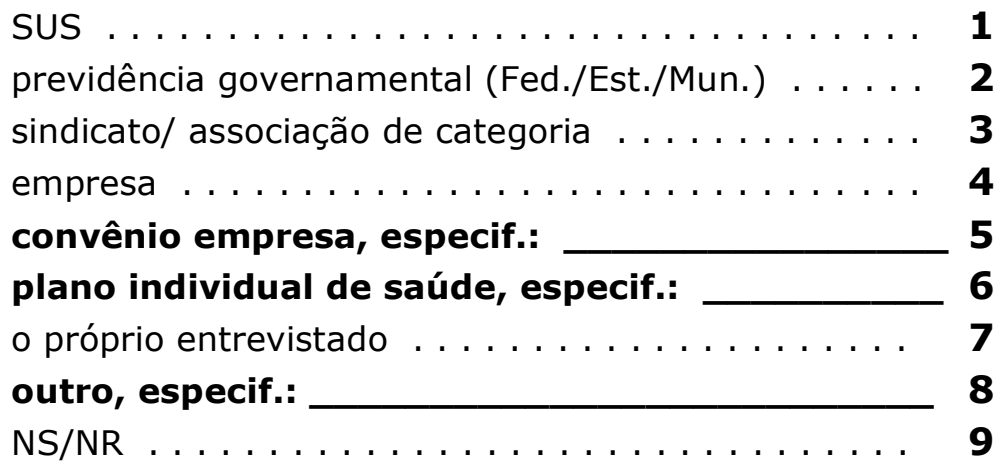

C 31. O senhor teve algum problema na realização de algum desse(s) exame(s)? passe $p$ / questão $\mathbf{C} \mathbf{3 4 .}$ 용

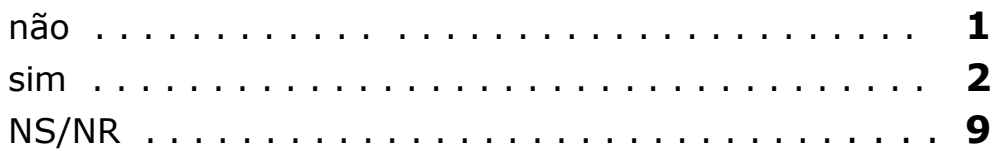

C 32. Na realização de qual(is) exame(s)?

Pode haver mais de uma resposta

(leia as alternativas para o entrevistado)

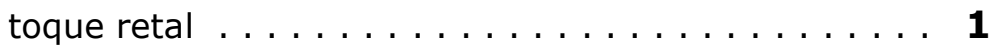

exame de sangue $(\mathrm{PSA}) \ldots \ldots \ldots \ldots \ldots \ldots$

ultra-sonografia $\ldots \ldots \ldots \ldots \ldots \ldots \ldots \ldots$

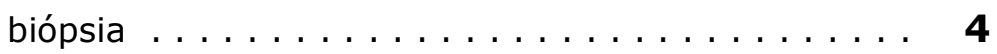

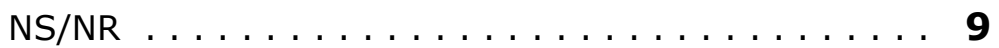

C 33. Que problema(s) teve? 
C 34. Existem exames utilizados nos programas de prevenção de câncer de intestino. O sr. já fez algum exame com esta finalidade?

passe $p /$ o BLOCO D.

影 não $\ldots \ldots \ldots \ldots \ldots \ldots \ldots \ldots \ldots \ldots \ldots$

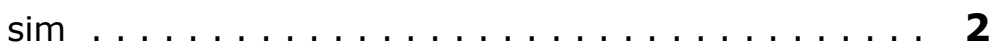

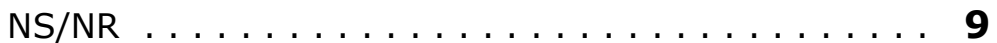

C 35. Qual(is) exame(s) realizou?

Pode haver mais de uma resposta

fez pesquisa de sangue oculto nas fezes . . . . . 1

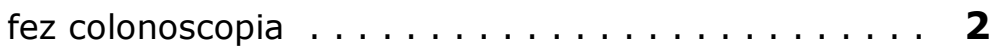

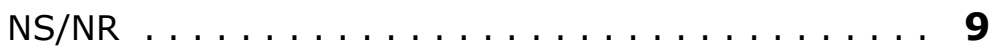

C 36. Quando foi a última vez que o sr. fez algum desses exames?

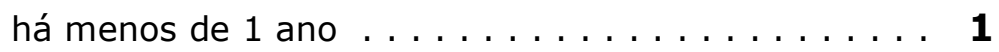

há 1 a 2 anos incompletos ............. 2

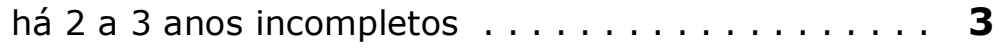

há mais de 3 anos . . . . . . . . . . . 4

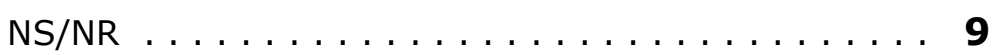

C 37. Em que serviço de saúde o sr. fez o(s) exame(s)?

unidade básica de saúde

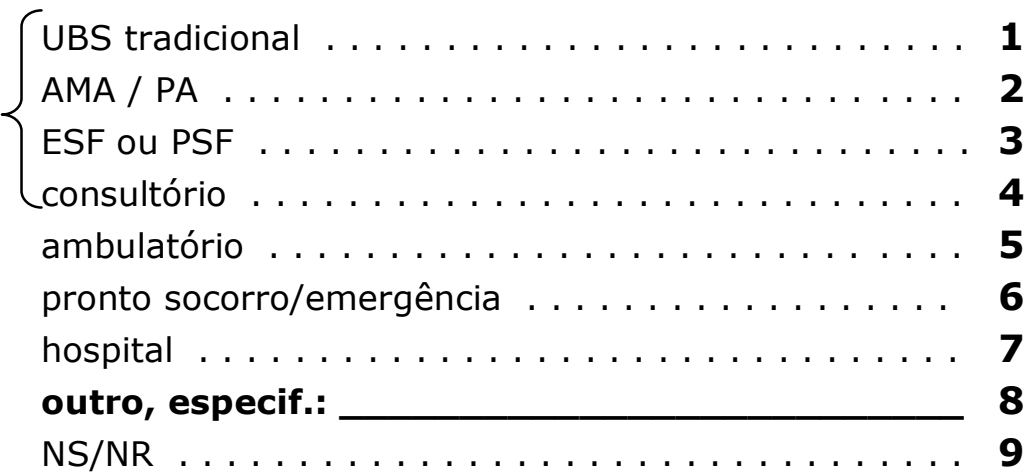


C 38. Esse serviço de saúde é: (leia as alternativas para o entrevistado)

$\begin{array}{ll}\text { SUS } \ldots \ldots \ldots \ldots & \mathbf{1} \\ \text { previdência governamental (Fed./Est./Mun.) } \ldots \ldots \ldots & \mathbf{2} \\ \text { privado/particular } \ldots \ldots \ldots \ldots \ldots \ldots \ldots \ldots \ldots \ldots \ldots & \mathbf{3} \\ \text { serviço de sindicato/ associação de categoria } \ldots \ldots \ldots & \mathbf{4} \\ \text { serviço próprio de empresa } \ldots \ldots \ldots \ldots \ldots \ldots & \mathbf{5} \\ \text { outro, especif.: } \ldots \ldots \ldots \ldots & \mathbf{6} \\ \text { NS/NR } \ldots \ldots \ldots \ldots \ldots & \end{array}$

C 39. Quem cobriu ou complementou os gastos deste(s) exame(s)?

Pode haver mais de uma resposta

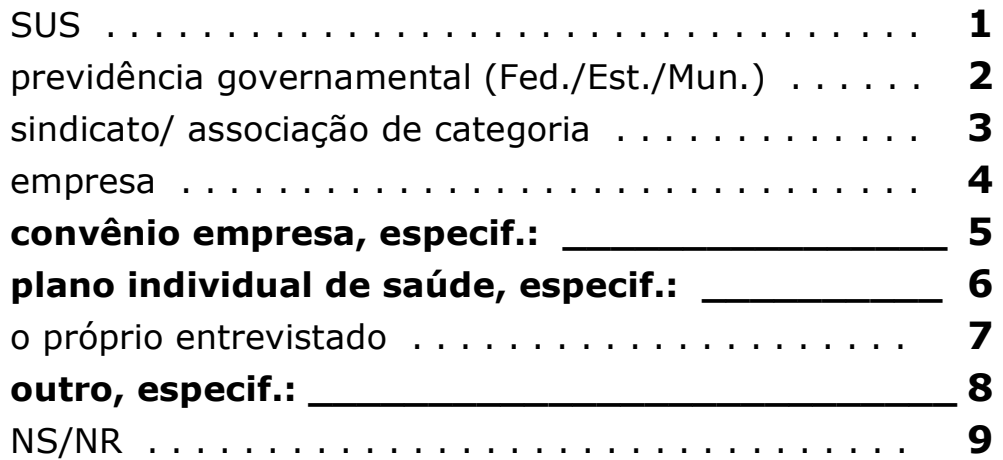

C 40. 0 sr. teve algum(ns) problema(s) na realização desse(s) exame(s)?

passe $p /$ o BLOCO D. ह

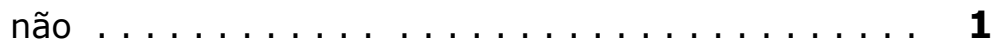

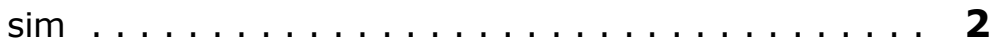

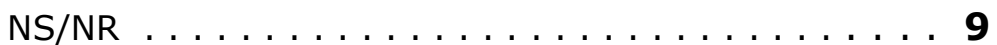

C 41. Que problema(s) teve? 
Doenças Crônicas

BLOCO D

D 01. Algum profissional de saúde já lhe disse que o(a) sr.(a) tem pressão alta? passe p/ questão D 06.

할

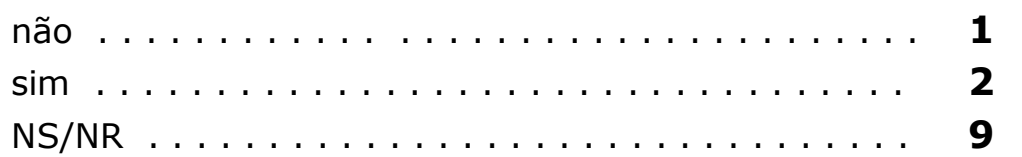

D 01

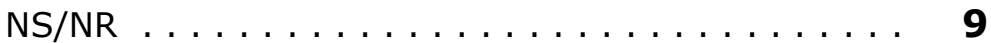

D 02. O(a) sr.(a) participa ou já participou de discussões de grupo sobre controle de pressão alta?

passe $p /$ questão D 06.

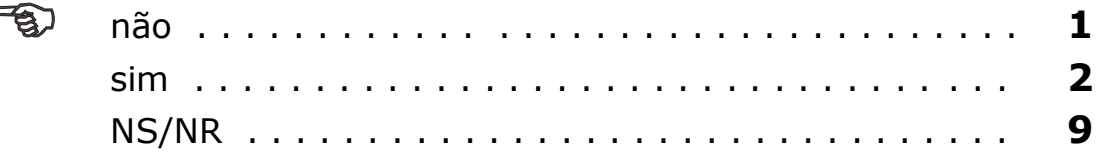

D 03. Com que freqüência o(a) sr(a). participa dessas reuniões?

foi somente uma vez $\ldots \ldots \ldots \ldots \ldots \ldots \ldots$

semanalmente ................ 2

quinzenalmente $\ldots \ldots \ldots \ldots \ldots \ldots \ldots$

mensalmente .................4 4

bimestralmente ................ 5

sem freqüência definida $\ldots \ldots \ldots \ldots \ldots \ldots$

outro, especif.: _ 7

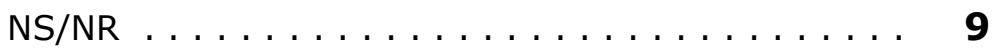

D 04. Essa(s) atividade(s) é(são) voltada(s) especificamente para pessoas com a sua deficiência?

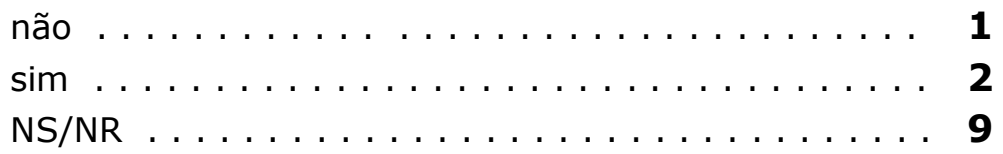

D 05. O(a) sr(a). tem algum problema para compreender as informações passadas nessas atividades?

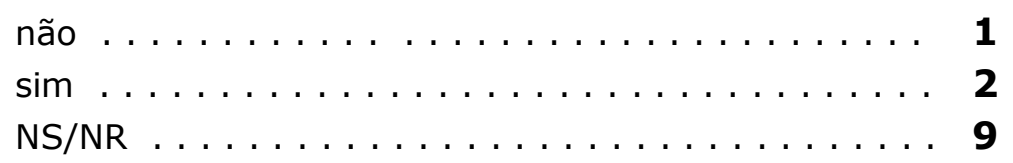


D 06. Algum profissional de saúde já lhe disse que o(a) sr.(a) tem diabetes? passe $p /$ questão D 11.

ह

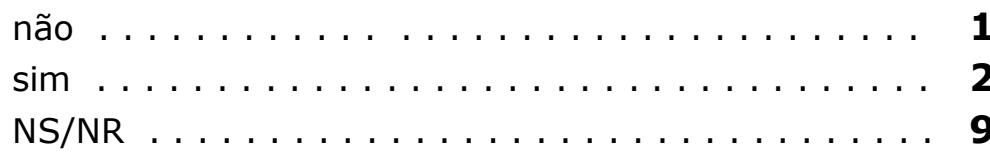

D 07. $O(a)$ sr.(a) participa ou já participou de discussões de grupo sobre controle do diabetes? passe $p /$ questão D $\mathbf{1 1}$. है:

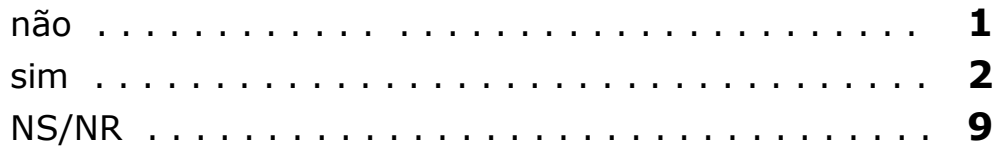

D 08. Com que freqüência o(a) sr(a). participa dessas reuniões?

foi somente uma vez . . . . . . . . . . . 1

semanalmente ................. 2

quinzenalmente $\ldots \ldots \ldots \ldots \ldots \ldots \ldots \ldots \ldots \ldots \ldots$

mensalmente ................. 4

bimestralmente ...............5

sem freqüência definida $\ldots \ldots \ldots \ldots \ldots \ldots$

outro, especif.: _ 7

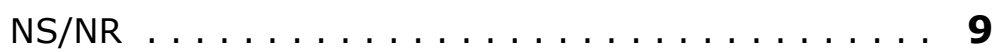

D 09. Essa(s) atividade(s) é(são) voltada(s) especificamente para pessoas com a sua deficiência?

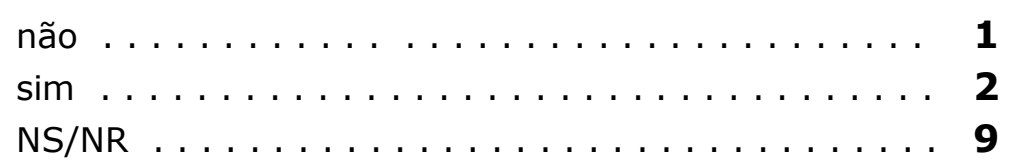

D 10. $O(a) \operatorname{sr}(a)$. tem algum problema para compreender as informações passadas nessas atividades?

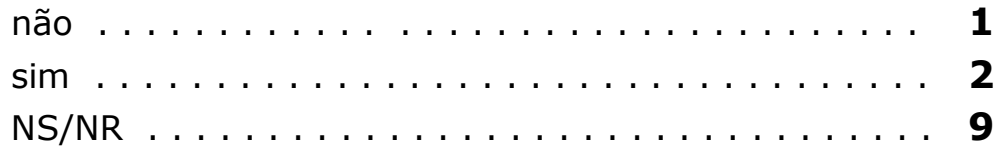

D 11. No seu atendimento em saúde o(a) sr.(a) tem participado ou já participou de alguma atividade educativa ou de orientação sobre cuidados pessoais referentes à sua deficiência?

não

sim

NS/NR
1 
D 12. Com que freqüência o(a) sr(a). participa dessas atividades?

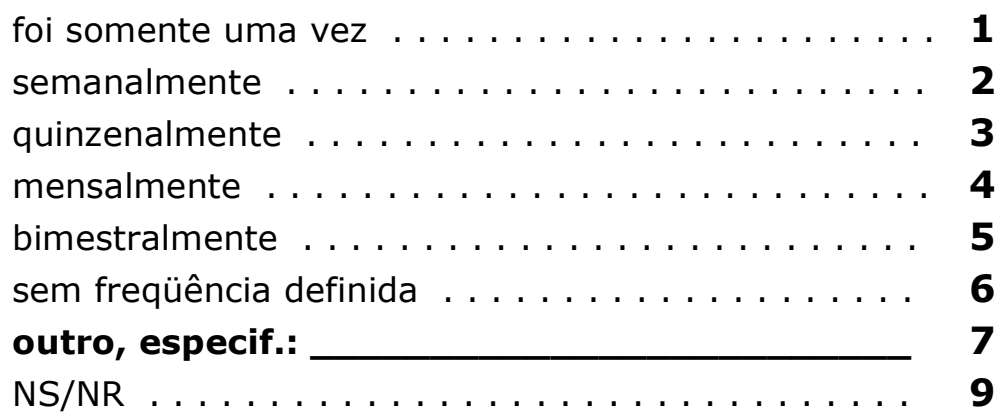

D 13. $O(a) \operatorname{sr}(a)$. tem algum problema para compreender as informações passadas nessas atividades?

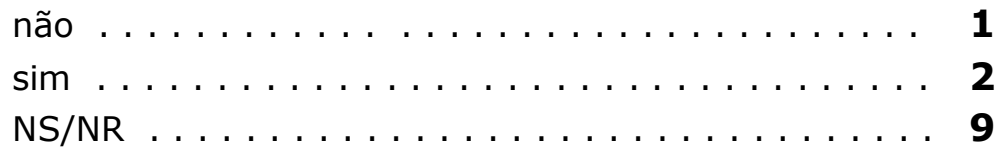

D 14. Por causa da sua deficiência, o(a) sr.(a) teve algum problema para freqüentar palestras, grupos ou campanhas?

encerre e leia a chamada

ध्ध

não

1

no final do bloco

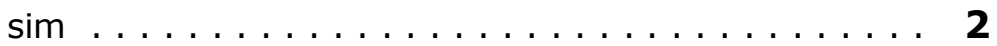

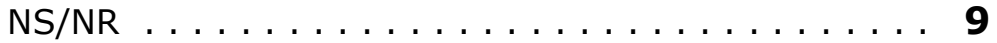

D 15. Que problema(s) teve?

NS/NR 999

Se o entrevistado for deficiente auditivo, responda o bloco E1 e $\boldsymbol{F}$.

Se o entrevistado for deficiente visual, responda o bloco E2.

Se o entrevistado for deficiente físico, passe para o bloco G. 
Deficiente Auditivo

Exames Auditivos

E1-01. Quando o(a) sr(a). fez exame de audição pela última vez?

BLOCO E1

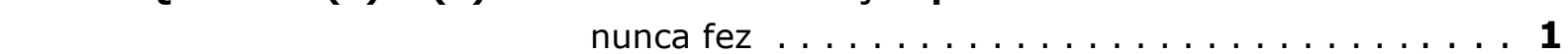

passe $p /$ questão E1-03.

E1-01

passe $p /$ questão E1-03. há 1 a 2 anos incompletos $\ldots \ldots \ldots \ldots \ldots \ldots \ldots$

há 2 a 3 anos incompletos . . . . . . . . 4

há mais de 3 anos .............. 5

NS/NR ................. 9

E1-02. Por que não fez este exame nos últimos anos?

Pode haver mais de uma resposta

porque não sabia onde procurar o serviço . . . . . . 01

não tinha o profissional/serviço que precisava . . . . . 02

não conseguiu vaga/senha . . . . . . . . . 03

o serviço não estava funcionando . . . . . . . . . . 04

esperou muito e desistiu . . . . . . . . . . . 05

porque nunca foi pedido pelo médico . . . . . . . 06

porque não podia pagar . . . . . . . . . . . 07

porque o plano de saúde não cobria $\ldots \ldots \ldots \ldots .08$

problemas de comunicação com os profissionais de

saúde ........ 09

porque não tinha tempo $\ldots \ldots \ldots \ldots \ldots \ldots \ldots$

outro, especif.: _ـ 11

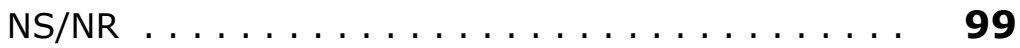

E1-03. O(a) sr(a). alguma vez teve algum problema com a realização desse exame? encerre e leia a chamada

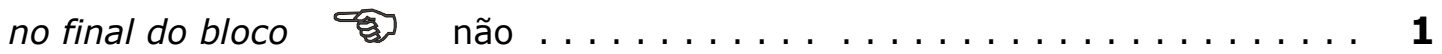

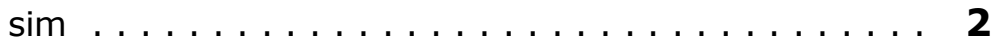

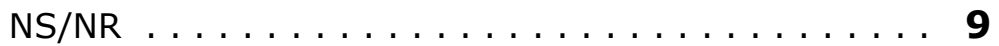

E1-04. Que problema(s) teve?

NS/NR

Se o entrevistado for também deficiente visual, responda o BLOCO E2., caso contrário passe para o BLOCO F. 
Deficiente Visual

Exames Visuais

BLOCO E2

E2-01. Quando o(a) sr(a). fez exame de visão pela última vez?

nunca fez ................. 1

E2-01

passe $p /$ questão E2-03.

há menos de 1 ano

2

passe $p /$ questão E2-03. há 1 a 2 anos incompletos $\ldots \ldots \ldots \ldots \ldots \ldots$

há 2 a 3 anos incompletos ............. 4

há mais de 3 anos .............. 5

NS/NR ................. 9

E2-02. Por que não fez este exame nos últimos anos?

Pode haver mais de uma resposta

porque não sabia onde procurar o serviço . . . . . . 01

não tinha o profissional/serviço que precisava . . . . 02

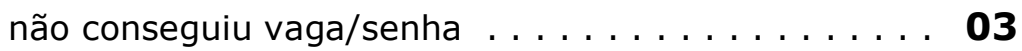

o serviço não estava funcionando . . . . . . . . . . . 04

esperou muito e desistiu . . . . . . . . . . . 05

porque nunca foi pedido pelo médico $\ldots \ldots \ldots \ldots 06$

porque não podia pagar . . . . . . . . . . 07

porque o plano de saúde não cobria $\ldots \ldots \ldots \ldots \ldots \mathbf{0 8}$

problemas de comunicação com os profissionais de

saúde ......... 09

porque não tinha tempo $\ldots \ldots \ldots \ldots \ldots \ldots \ldots$

outro, especif.: _ 11

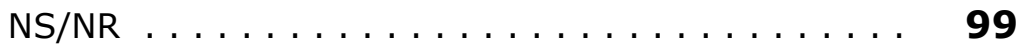

E2-03. $O(a)$ sr(a). alguma vez teve algum problema com a realização desse exame? encerre e leia a chamada

no final do bloco o não . . . . . . . . . . . . . . . . . . 1

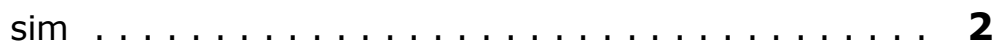

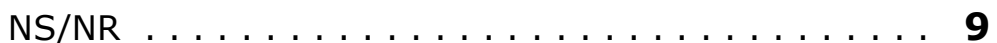

E2-04. Que problema(s) teve?

NS/NR $\ldots \ldots \ldots \ldots \ldots \ldots \ldots \ldots \ldots$ 
Deficiente Auditivo

Comunicação e Atendimento

Agora vamos falar sobre os problemas na comunicação em seu atendimento no último serviço de saúde que o(a) $\operatorname{sr}(a)$. procurou.

F 01. Em decorrência de sua dificuldade de audição, $o(a) \operatorname{sr}(a)$. teve algum F 01 problema em entender o que o médico lhe dizia?

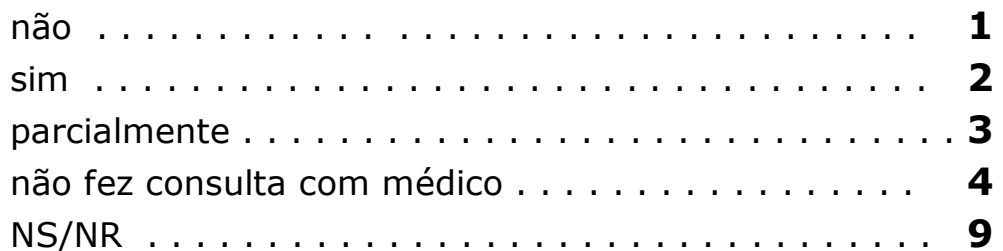

F 02. $O(a) \operatorname{sr}(a)$. teve algum problema para se fazer entender pelo médico?

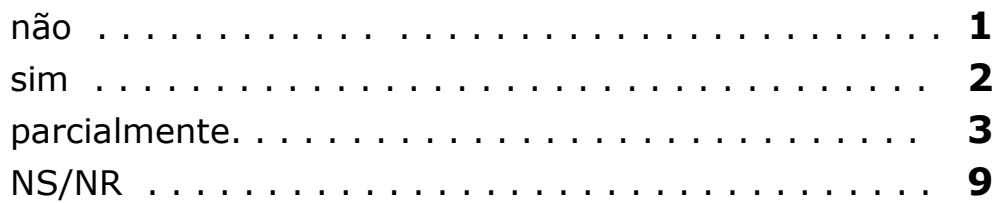

F 03. $O(a) \operatorname{sr}(a)$. considera que o médico que o(a) atendeu estava preparado para a comunicação com pacientes com deficiência auditiva?

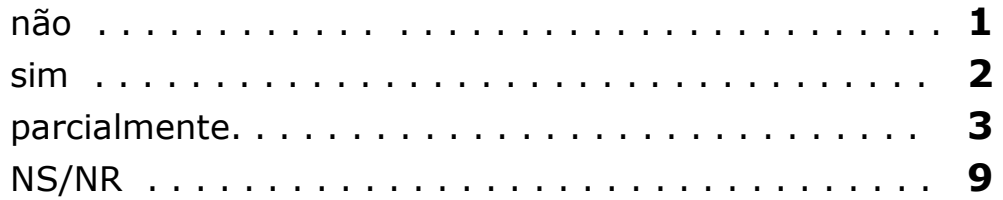

F 04. Em decorrência de sua dificuldade auditiva, o(a) sr(a). teve algum problema em entender o que foi falado ou explicado pelo(s) enfermeiro(s)?

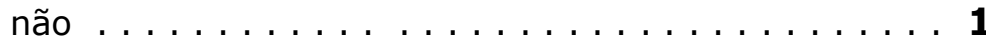

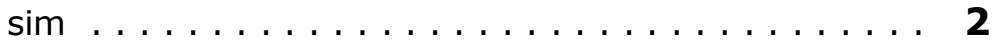

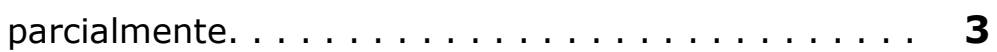

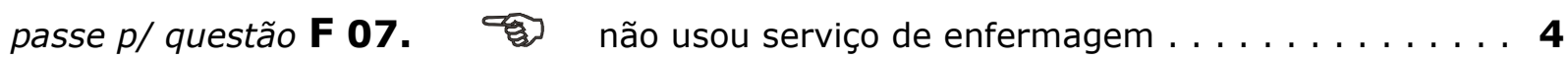

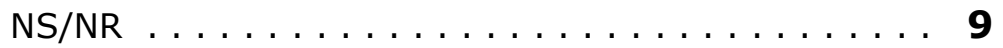

F 05. O(a) sr(a). teve algum problema para se fazer entender pelo(s) enfermeiro(s)?

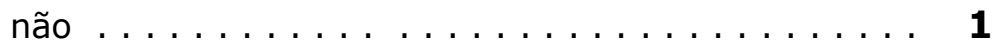

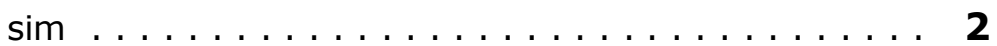

parcialmente. . . . . . . . . . . . . 3

NS/NR $\ldots \ldots \ldots \ldots \ldots \ldots \ldots \ldots \ldots \ldots$ 
F 06. $O(a) \operatorname{sr}(a)$. considera que o(s) enfermeiros(s) que o(a) atendeu(ram) estava(m) preparado(s) para a comunicação com pacientes com deficiência auditiva?

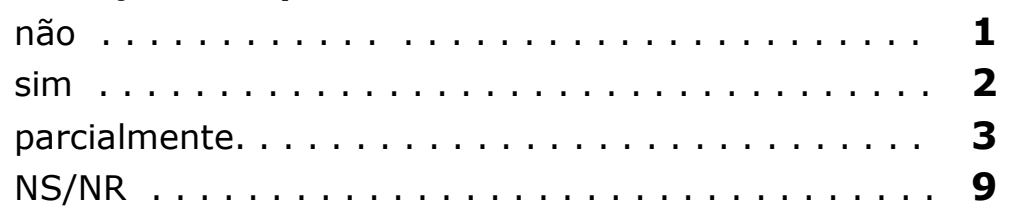

F 07. Em decorrência de sua dificuldade auditiva, $o(a) \operatorname{sr}(a)$. teve algum problema em entender o que foi falado ou explicado pelo(s) funcionário(s), atendentes(s) e recepcionista(s)?

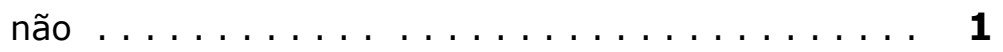

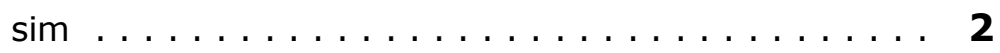

parcialmente. ................ 3

passe $p /$ questão F 10. não teve contato com funcionários, atendentes e

recepcionistas .......... 4

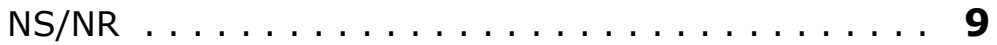

F 08. $O(a) \operatorname{sr}(a)$. teve algum problema para se fazer entender pelo(s) funcionário(s), atendentes(s) e recepcionista(s)?

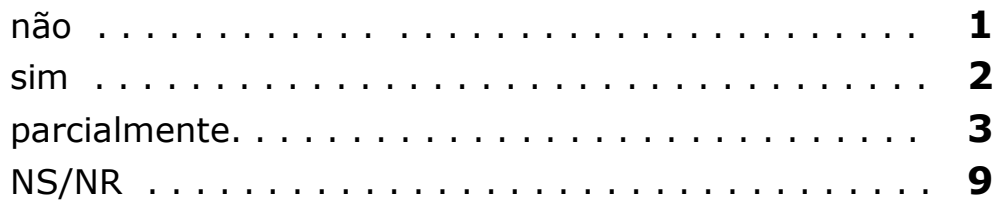

F 09. $O(a) \operatorname{sr}(a)$. considera que o(s) funcionário(s), atendentes(s)e recepcionista(s) que o(a) atendeu(ram) estava(m) preparado(s) para a comunicação com pacientes com deficiência auditiva?

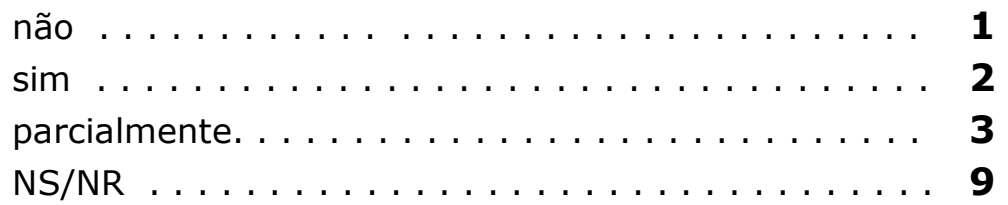

F 10. Existem outro(s) problema(s) de comunicação com os profissionais de saúde que o(a) sr.(a) queira relatar? passe $p / o$ BLOCO G.

है não 1

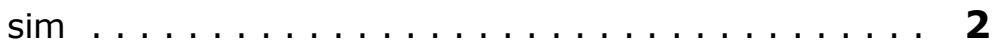

NS/NR $\ldots \ldots \ldots \ldots \ldots \ldots \ldots \ldots \ldots$

F 11. Que problema(s) teve? 
Necessidade de Saúde Não Suprida

BLOCO G

G 01. Nos últimos 12 meses, o(a) sr(a). precisou atendimento em algum serviço de $\quad$ G 01 saúde? E conseguiu?

passe $p /$ questão $\mathbf{G}$ 04.

sim, precisei e consegui atendimento. . . . . . . . 2

sim, precisei e não consegui atendimento . . . . . . 3

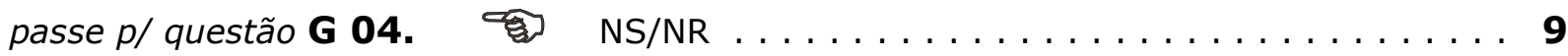

G 02. Qual(is) atendimento(s) precisou?

NS/NR $\ldots \ldots \ldots \ldots \ldots \ldots \ldots \ldots$

pular para a questão G 04. se conseguiu atendimento.

G 03. Por que o(a) sr(a). não conseguiu o atendimento?

Pode haver mais de uma resposta

porque não sabia onde procurar o serviço . . . . . 01

não tinha o profissional/serviço que precisava . . . . 02

não conseguiu vaga/senha $\ldots \ldots \ldots \ldots \ldots \ldots$

o serviço não estava funcionando . . . . . . . . . . 04

esperou muito e desistiu . . . . . . . . . . . . 05

porque não podia pagar $\ldots \ldots \ldots \ldots \ldots \ldots \ldots$

porque o plano de saúde não cobria $\ldots \ldots \ldots \ldots \ldots \mathbf{0 7}$

problemas de comunicação com os profissionais de

saúde .......... 08

não conseguiu se deslocar até o serviço de saúde . . 09

problemas com rampas e escadas no serviço de

saúde. . . . . . . . 10

outro, especif.: _ 11

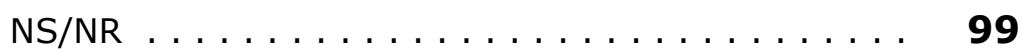

G 04. Nos últimos 12 meses, o(a) sr(a). precisou de atendimento médico no domicílio? E conseguiu?

sim precisei e não consegui atendimento . . . . . $\mathbf{1}$

passe $p$ / questão G 06. $\left\{\begin{array}{l}\text { não precisei de atendimento } \ldots \ldots \ldots \ldots \ldots \ldots \ldots \ldots \ldots \ldots \\ \text { sim, precisei e consegui atendimento } \ldots \ldots \ldots \ldots \ldots \ldots \ldots \\ \text { NS/NR } \ldots \ldots \ldots \ldots \ldots\end{array}\right.$ 
G 05. Por que o(a) sr(a). não conseguiu o atendimento?

Pode haver mais de uma resposta

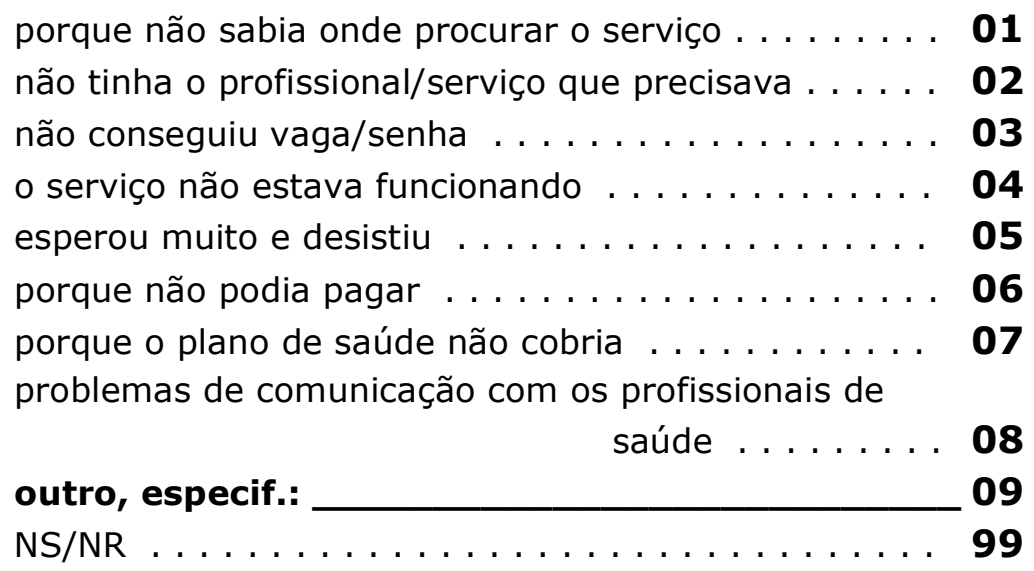

G 06. Nos últimos 12 meses, o(a) sr(a). precisou de algum atendimento de enfermeiros no domicílio? E conseguiu?

sim precisei e não consegui atendimento . . . . . . . . $\mathbf{1}$

passe $p$ / questão G 08. $\left\{\begin{array}{l}\text { não precisei de atendimento } \ldots \ldots \ldots \ldots \ldots \ldots \ldots \ldots \ldots \ldots \ldots \ldots \ldots \\ \text { sim, precisei e consegui atendimento } \ldots \ldots \ldots \ldots \ldots \ldots \\ \text { NS/NR } \ldots \ldots \ldots \ldots\end{array}\right.$

G 07. Por que o(a) sr(a). não conseguiu o atendimento?

Pode haver mais de uma resposta

porque não sabia onde procurar o serviço . . . . . . 01

não tinha o profissional/serviço que precisava . . . . . 02

não conseguiu vaga/senha . . . . . . . . . . 03

o serviço não estava funcionando . . . . . . . . . . . 04

esperou muito e desistiu . . . . . . . . . . 05

porque não podia pagar $\ldots \ldots \ldots \ldots \ldots 6$

porque o plano de saúde não cobria $\ldots \ldots \ldots \ldots \ldots 7$

problemas de comunicação com os profissionais de

saúde ....... 08

outro, especif.:

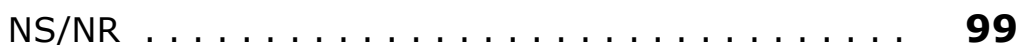

G 08. Nos últimos 12 meses, $o(a) \operatorname{sr}(a)$. precisou de algum outro atendimento por profissionais de saúde no domicílio? E conseguiu?

passe $p /$ questão $\mathbf{G} \mathbf{1 1 .}$

passe $p /$ questão G 11. não precisei de atendimento . . . . . . . . . 1

sim, precisei e consegui atendimento. . . . . . . . 2

sim, precisei e não consegui atendimento . . . . . . 3

ह $\mathrm{NS} / \mathrm{NR} \ldots \ldots \ldots \ldots \ldots \ldots \ldots$ 
G 09. Qual(is) atendimento(s) precisou?

NS/NR $\ldots \ldots \ldots \ldots \ldots \ldots \ldots \ldots$

pular para a questão $\mathbf{G} \mathbf{1 0}$. se conseguiu atendimento.

G 10. Por que o(a) sr(a). não conseguiu o atendimento?

Pode haver mais de uma resposta

$\begin{array}{lll}\text { porque não sabia onde procurar o serviço } \ldots \ldots \ldots & 01 \\ \text { não tinha o profissional/serviço que precisava } \ldots \ldots & 02\end{array}$

não conseguiu vaga/senha $\ldots \ldots \ldots \ldots \ldots \ldots$

o serviço não estava funcionando $\ldots \ldots \ldots \ldots \ldots$

esperou muito e desistiu . . . . . . . . . . . 05

porque não podia pagar $\ldots \ldots \ldots \ldots \ldots$

porque o plano de saúde não cobria $\ldots \ldots \ldots \ldots . . \ldots 7$

problemas de comunicação com os profissionais de

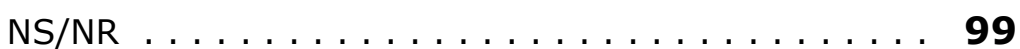

G 11. Nos últimos 12 meses, o(a) sr(a). precisou consultar dentista, inclusive visita de rotina? E conseguiu?

sim precisei e não consegui atendimento . . . . . . $\mathbf{1}$

passe $p /$ questão G 13. $\left\{\begin{array}{l}\text { não precisei de atendimento } \ldots \ldots \ldots \ldots \ldots \ldots \ldots \ldots \ldots \ldots \\ \text { sim, precisei e consegui atendimento } \ldots \ldots \ldots \ldots \ldots \ldots \\ \text { NS/NR } \ldots \ldots \ldots \ldots \ldots\end{array}\right.$

G 12. Por que o(a) sr(a). não conseguiu a consulta?

Pode haver mais de uma resposta

porque não sabia onde procurar o serviço . . . . . . 01

não tinha o profissional/serviço que precisava . . . . 02

não conseguiu vaga/senha . . . . . . . . . . 03

o serviço não estava funcionando . . . . . . . . . 04

esperou muito e desistiu . . . . . . . . . . 05

porque não podia pagar $\ldots \ldots \ldots \ldots \ldots$

porque o plano de saúde não cobria $\ldots \ldots \ldots \ldots . . \ldots 7$

problemas de comunicação com os profissionais de

saúde . . . . . . . 08

outro, especif.: $\_09$

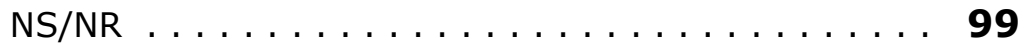


G 13. Nos últimos 12 meses, o(a) sr(a). deixou de obter algum medicamento que Ihe foi receitado?

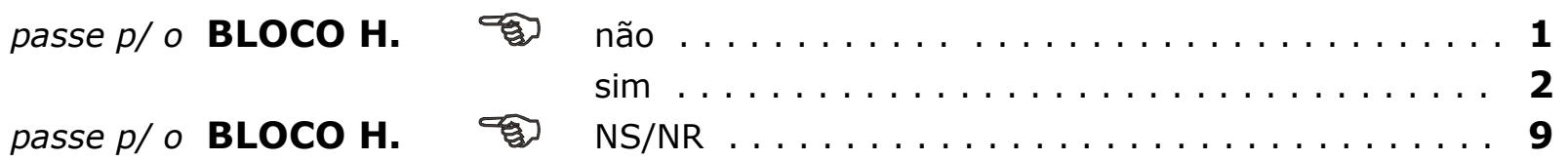

G 14. Por que o(a) sr(a). não conseguiu obtê-lo?

Pode haver mais de uma resposta

porque não sabia onde procurar o medicamento . . . $\mathbf{1}$

o serviço não estava funcionando . . . . . . . . 2

porque não podia pagar . . . . . . . . . 3

porque o plano de saúde não cobria $\ldots \ldots \ldots \ldots \ldots 4$

porque não tinha no posto $\ldots \ldots \ldots \ldots \ldots \ldots \ldots \mathbf{5}$

outro, especif.: _ 6

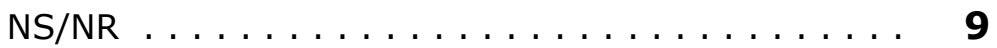


Programa de Saúde da Família

BLOCO H

H 01. O(a) sr(a). utiliza o Programa de Saúde da Família (PSF/ESF)?

passe p/ questão H 03.

है $\mathrm{n}$

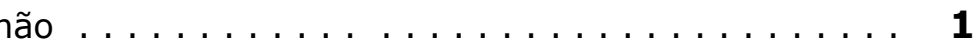

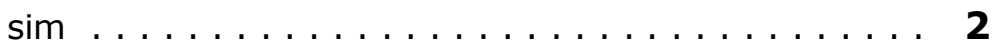

encerre e leia a chamada

NS/NR

9 no final do bloco

H 02. O(a) sr(a). já precisou ser atendido pelo Programa de Saúde da Família (PSF/ESF) e não foi?

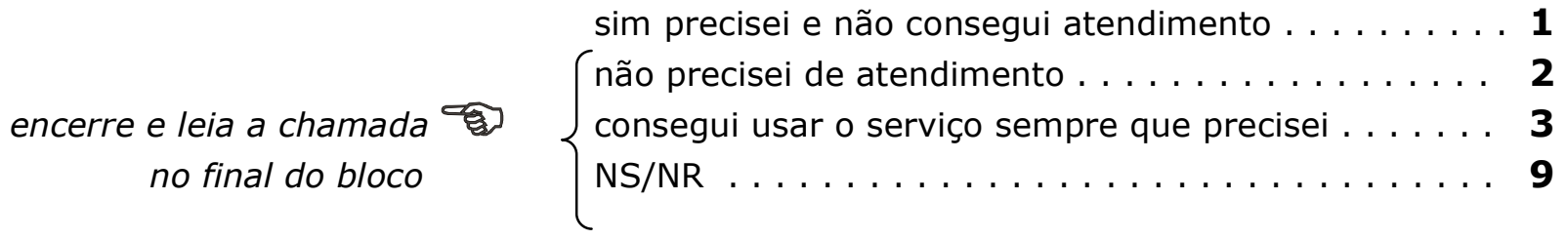

H 03. Por quê?

porque minha casa não está na área de cobertura . . . . 1 porque não havia especialistas no problema de saúde . $\mathbf{2}$ porque não havia disponibilidade de tempo da equipe . $\mathbf{3}$ outro, especif.: 4

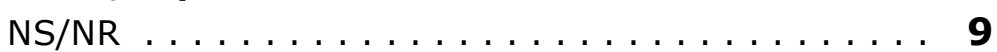

Se o entrevistado for deficiente auditivo, responda o bloco I.

Se o entrevistado for deficiente visual, responda o bloco J.

Se o entrevistado for deficiente físico, responda o bloco $\mathrm{K}$. 
Deficiente Auditivo

Próteses, Órteses e Outros Equipamentos/Insumos

BLOCO I

I 01. O(a) $\operatorname{sr}(a)$. precisa usar aparelho auditivo?

encerre e leia a chamada

no final do bloco não $\ldots \ldots \ldots \ldots \ldots \ldots \ldots \ldots \ldots \ldots$

$\operatorname{sim} \ldots \ldots \ldots \ldots \ldots \ldots \ldots \ldots$

encerre e leia a chamada

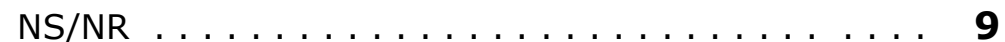

no final do bloco

I 02. $O(a) \operatorname{sr}(a)$. usa o aparelho?

não $\ldots \ldots \ldots \ldots \ldots \ldots \ldots \ldots \ldots \ldots$

passe p/ questão I 04.

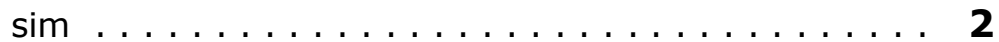

encerre e leia a chamada

NS/NR

9 no final do bloco

I 03. Porque não usa o aparelho?

NS/NR

encerre e leia a chamada no final do bloco

I 04. Como fez para obtê-lo?

Pode haver mais de uma resposta

comprou com dinheiro próprio . . . . . . . . . . . 1

a família pagou . . . . . . . . . . . . . . . . 2

doação de terceiros ou de entidades beneficentes . . . 3

recebeu do SUS . . . . . . . . . . . . . . . . 4

outro, especif.:

5

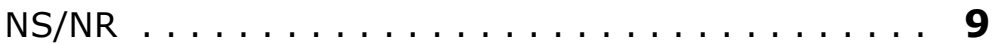

I 05. Se for necessária a troca, como fará para obter outro aparelho?

Pode haver mais de uma resposta

comprará com dinheiro próprio . . . . . . . . . . . . 1

a família pagará . . . . . . . . . . . . . . . . . 2

doação de terceiros ou de entidades beneficentes . . . 3

receberá do SUS . . . . . . . . . . . . . . . . . . 4

não fará a troca, não tem condições de pagar . . . . . . 5

outro, especif.:

6

NS/NR 
I 06. Se for necessária a troca de baterias, como fará?

Pode haver mais de uma resposta

comprará com dinheiro próprio . . . . . . . . $\mathbf{1}$

a família pagará . . . . . . . . . . . . 2

doação de terceiros ou de entidades beneficentes . . . 3

receberá do sus . . . . . . . . . . . . . . 4

não fará a troca, não tem condições de pagar . . . . . 5

outro, especif.: _ 6

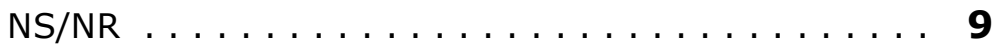

Se o entrevistado for também deficiente visual, responda o Bloco J.

Se o entrevistado for também deficiente físico, responda 0

Bloco K. 
Listarei agora alguns equipamentos ou próteses, $\mathrm{o}(a) \operatorname{sr}(a)$. dirá se precisa usar cada um deles:

Pode haver mais de uma resposta (leia as alternativas para o entrevistado)

\begin{tabular}{|c|c|c|c|c|c|c|c|c|}
\hline \multirow{8}{*}{\begin{tabular}{|c|} 
\\
encerreo \\
bloco e leia a \\
chamada final \\
\end{tabular}} & \multirow{2}{*}{$\begin{array}{l}\text { Precisa usar? } \\
\text { J } 01 \text { 1. óculos }\end{array}$} & \multirow{2}{*}{$\operatorname{sim}$} & \multirow{2}{*}{$\begin{array}{c}\text { não } \\
2\end{array}$} & \multirow{2}{*}{$\begin{array}{l}\text { Usa? } \\
\text { J 1. óculos }\end{array}$} & \multirow{2}{*}{$\operatorname{sim}$} & \multirow{2}{*}{2} & \multirow{2}{*}{$\begin{array}{l}\text { Porque não usa? } \\
\text { J } 03 \text { 1. óculos }\end{array}$} & \multirow{2}{*}{ 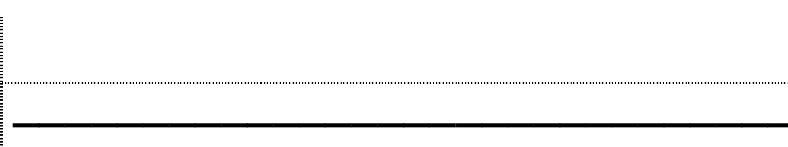 } \\
\hline & & & & & & & & \\
\hline & $\begin{array}{l}\text { J } 01 \text { 2. lente de } \\
\text { contato }\end{array}$ & 1 & 2 & $\begin{array}{l}\text { J } 02 \text { 2. lente de } \\
\text { contato }\end{array}$ & 1 & 2 & $\begin{array}{l}\int 032 \text {. lente de } \\
\text { contato }\end{array}$ & \\
\hline & $\begin{array}{l}\text { J } 01 \text { 3. lente de } \\
\text { aumento }\end{array}$ & 1 & 2 & $\begin{array}{l}\text { J } 02 \text { 3. lente de } \\
\text { aumento }\end{array}$ & 1 & 2 & $\begin{array}{l}\text { J } 03 \mathbf{3} \text {. lente de } \\
\text { aumento }\end{array}$ & \\
\hline & $\begin{array}{l}\text { J } 01 \text { 4. próteses } \\
\text { de olho }\end{array}$ & 1 & 2 & $\begin{array}{l}\text { J } 02 \text { 4. próteses } \\
\text { de olho }\end{array}$ & 1 & 2 & $\begin{array}{l}\text { J } 03 \text { 4. próteses } \\
\text { de olho }\end{array}$ & \\
\hline & J 01 5. bengala & 1 & 2 & J 02 5. bengala & 1 & 2 & J 03 5. bengala & \\
\hline & $\begin{array}{lll}\text { J } & \mathbf{0 1} & \mathbf{6 .} \\
\text { outro: } & \\
\end{array}$ & 1 & 2 & $\begin{array}{lll}0 & 02 & 6 . \\
\end{array}$ & 1 & 2 & J 03 & \\
\hline & J 01 9. NS/NR & 9 & 9 & J 02 9. NS/NR & 9 & 9 & & \\
\hline
\end{tabular}

Se não precisa usar nenhum, encerre e leia a chamada no final do bloco. Se usar todos os equipamentos que precisa, passe para a questão J 04. 
J 04. Como fez para obtê-lo(s)?

Pode haver mais de uma resposta

comprou com dinheiro próprio . . . . . . . . $\mathbf{1}$

a família pagou . . . . . . . . . . . . 2

doação de terceiros ou de entidades beneficentes . . 3

recebeu do sus . . . . . . . . . . . . . 4

alugou .................. 5

emprestou de alguém . . . . . . . . . . 6

outro, especif.:

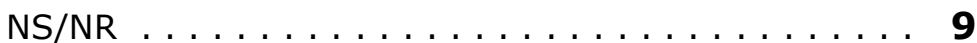

J 05. Se for necessária a troca, como fará para obter outro aparelho?

Pode haver mais de uma resposta

comprará com dinheiro próprio . . . . . . . . . 1

a família pagará . . . . . . . . . . . . 2

doação de terceiros ou de entidades beneficentes . . . 3

receberá do sus . . . . . . . . . . . . . 4

alugará . . . . . . . . . . . . . . . 5

emprestará de alguém . . . . . . . . . . . 6

não fará a troca, não tem condições de pagar . . . . . 7

outro, especif.: __ 8

NS/NR $\ldots \ldots \ldots \ldots \ldots \ldots \ldots \ldots$

Se o entrevistado for também deficiente auditivo, certifique-se que respondeu o Bloco $I$.

Se o entrevistado for também deficiente físico, responda 0

Bloco K. 
Listarei agora alguns equipamentos ou próteses, $\mathrm{o}(a) \operatorname{sr}(a)$. dirá se precisa usar cada um deles: Pode haver mais de uma resposta (leia as alternativas para o entrevistado)

\begin{tabular}{|c|c|c|c|c|c|c|c|c|}
\hline & Precisa usar? & $\operatorname{sim}$ & não & Usa? & $\operatorname{sim}$ & não & Porque não usa? & \\
\hline & K 01 1. muletas & 1 & 2 & K 02 1. muletas & 1 & 2 & K 03 1. muletas & \\
\hline & K 01 2. bengala & 1 & 2 & K 02 2. bengala & 1 & 2 & K03 2. bengala & \\
\hline & K 01 3. cadeira de rodas & 1 & 2 & $\begin{array}{l}\mathbf{K} 02 \text { 3. cadeira de } \\
\text { rodas }\end{array}$ & 1 & 2 & $\begin{array}{l}\mathbf{K} 03 \text { 3. cadeira de } \\
\text { rodas }\end{array}$ & \\
\hline & $\begin{array}{l}\text { K } 01 \text { 4. próteses (braço, } \\
\text { pernas, etc.) }\end{array}$ & 1 & 2 & K 02 4. Próteses & 1 & 2 & K 03 4. Próteses & \\
\hline & $\begin{array}{l}\mathbf{K} 01 \text { 5. Talas, goteiras, } \\
\text { palmilhas, etc. }\end{array}$ & 1 & 2 & 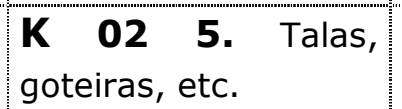 & 1 & 2 & 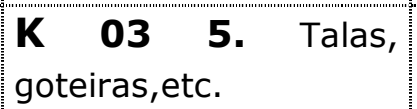 & \\
\hline & $\begin{array}{lll}\mathbf{K} & \mathbf{0 1} & \mathbf{6 .} \\
\text { outro: } & & \end{array}$ & 1 & 2 & 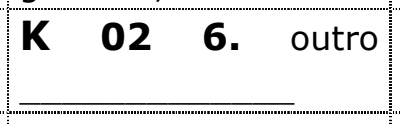 & 1 & 2 & $\begin{array}{llll}K & 03 & 6 . & \text { outro }\end{array}$ & \\
\hline asse $p / \mathbf{K} 06$. & K 01 9. NS/NR & 9 & 9 & K 02 9. NS/NR & 9 & 9 & & \\
\hline
\end{tabular}


Pode haver mais de uma resposta

comprou com dinheiro próprio $\ldots \ldots \ldots \ldots \ldots \ldots \ldots \mathbf{1}$

a família pagou ................. $\mathbf{2}$

doação de terceiros ou de entidades beneficentes . . . 3

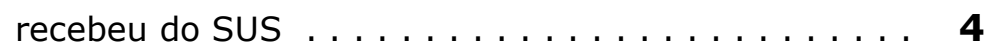

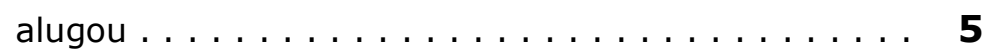

emprestou de alguém . . . . . . . . . 6

outro, especif.: __ 7

NS/NR $\ldots \ldots \ldots \ldots \ldots \ldots \ldots \ldots \ldots$

K 05. Se for necessária a troca, como fará para obter outro aparelho?

Pode haver mais de uma resposta

comprará com dinheiro próprio $\ldots \ldots \ldots \ldots \ldots \ldots$

a família pagará . . . . . . . . . . . . . 2

doação de terceiros ou de entidades beneficentes . . . 3

receberá do SUS . . . . . . . . . . . . 4

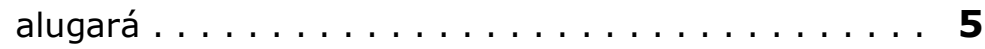

emprestará de alguém . . . . . . . . . . 6

não fará a troca, não tem condições de pagar . . . . . 7

outro, especif.: __ 8

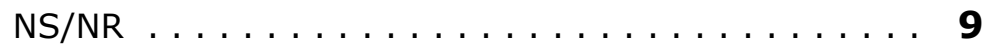


Listarei agora alguns outros equipamentos, o(a) sr(a). dirá se precisa usar cada um deles: Pode haver mais de uma resposta (leia as alternativas para o entrevistado)

\begin{tabular}{|c|c|c|c|c|c|c|c|c|}
\hline & \multirow{2}{*}{$\begin{array}{l}\text { Precisa usar? } \\
\text { K } 06 \text { 1. fraldas }\end{array}$} & \multirow{2}{*}{$\operatorname{sim}$} & \multirow{2}{*}{$\begin{array}{c}\text { não } \\
\mathbf{2}\end{array}$} & \multirow{2}{*}{$\begin{array}{r}\text { Usa? } \\
\text { K } 07 \text { 1. fraldas }\end{array}$} & \multirow{2}{*}{$\operatorname{sim}$} & \multirow{2}{*}{ não } & Porque não usa? & \multirow{2}{*}{ 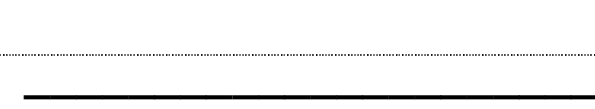 } \\
\hline & & & & & & & K 08 1. fraldas & \\
\hline & K 06 2. bolsas coletoras & 1 & 2 & K 07 2. bolsas coletoras & 1 & 2 & $\begin{array}{l}\mathbf{K} \\
\text { coletoras }\end{array}$ & \\
\hline & K 06 3. sondas & 1 & 2 & K 07 3. sondas & 1 & 2 & K 08 3. sondas & \\
\hline & $\begin{array}{l}\mathbf{K} 06 \text { 4. curativos ou } \\
\text { ataduras }\end{array}$ & 1 & 2 & $\begin{array}{l}\mathbf{K} 07 \text { 4. curativos ou } \\
\text { ataduras }\end{array}$ & 1 & 2 & $\begin{array}{l}\text { K } 08 \text { 4. curativos } \\
\text { ou ataduras }\end{array}$ & \\
\hline encerre o & $\begin{array}{lll}\mathbf{K} & 06 & 5 . \\
\text { outro: } & \end{array}$ & 1 & 2 & K 07 5. outro: & 1 & 2 & K 08 5. outro: & \\
\hline a chamada & K 06 9. NS/NR & 9 & 9 & K 07 9. NS/NR & 9 & 9 & & \\
\hline
\end{tabular}

Se não precisa usar nenhum, encerre e leia a chamada final. Se usa todos os insumos que precisa, passe para a $\mathrm{K} 09$. 
K 09. Como fez para obtê-lo(s)?

Pode haver mais de uma resposta

comprou com dinheiro próprio . . . . . . . . . . $\mathbf{1}$

a família pagou . . . . . . . . . . . . 2

doação de terceiros ou de entidades beneficentes . . 3

recebeu do SUS . . . . . . . . . . . . . 4

outro, especif.:

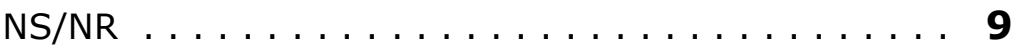

Se o entrevistado for também deficiente auditivo, certifique-se que respondeu o Bloco I. Se o entrevistado for também deficiente visual,

certifique-se que respondeu o Bloco J. 
8.3.ANEXO III - MATERIAL DE DIVULGAÇÃO DO CONSELHO MUNICIPAL DA PESSOA COM DEFICIÊNCIA

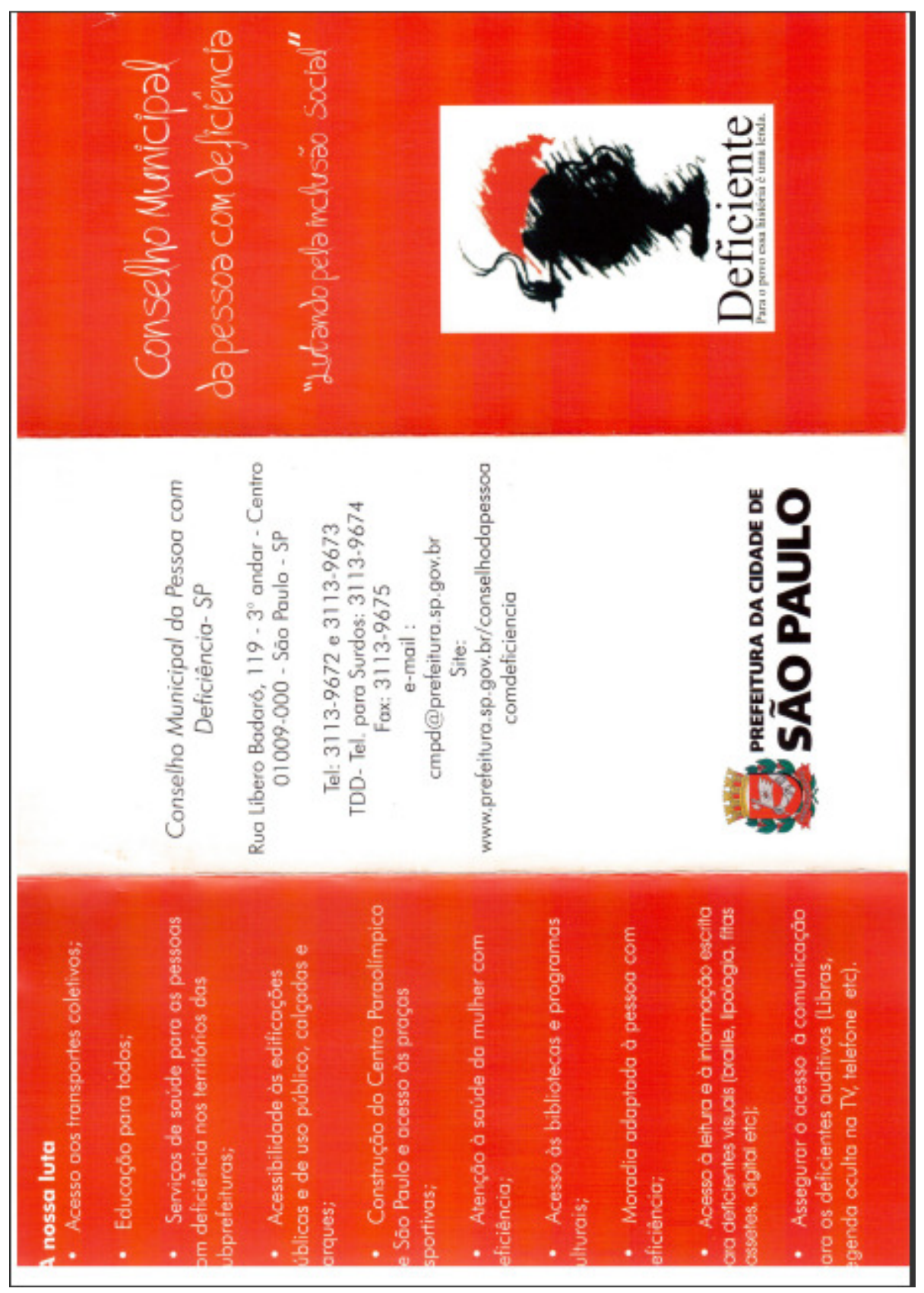




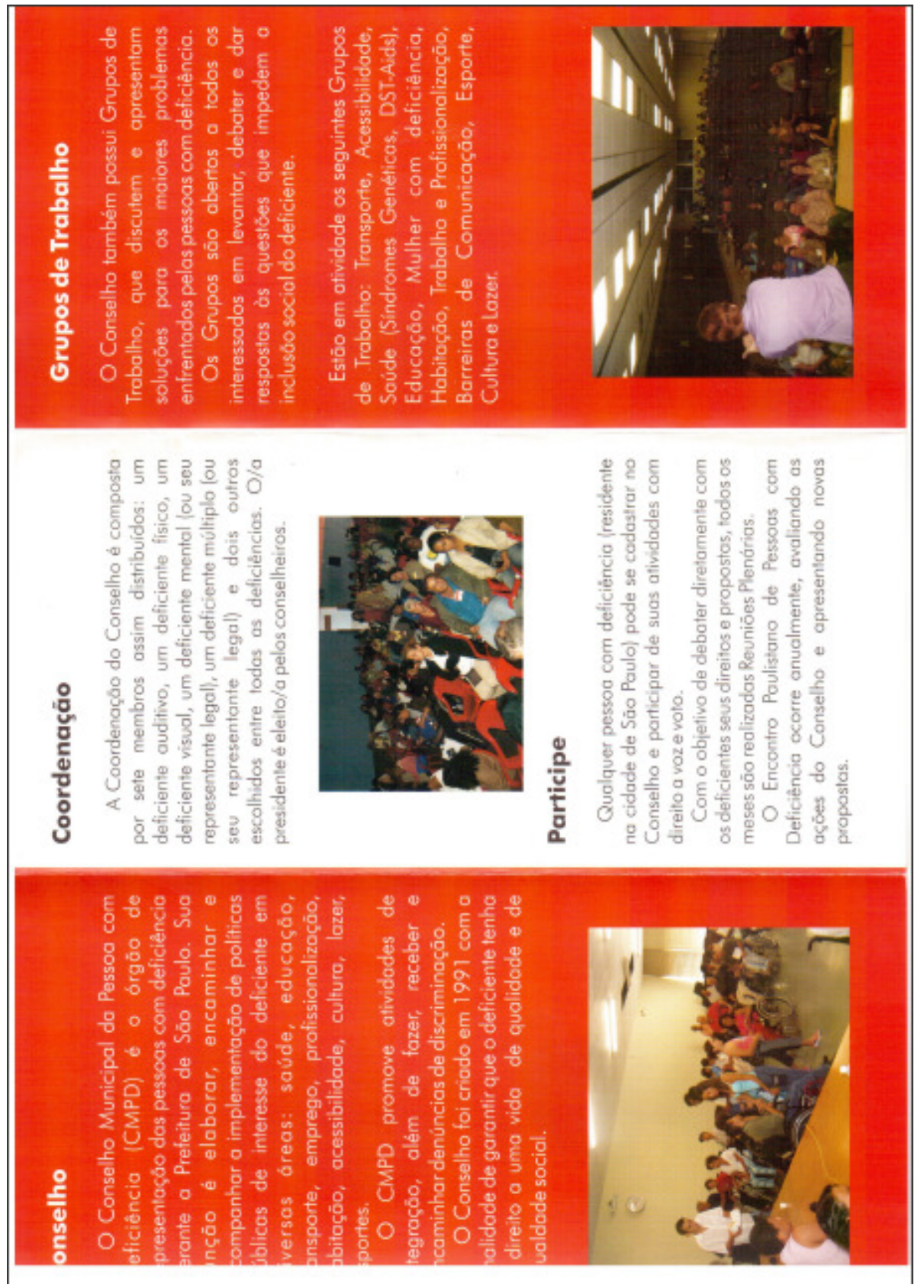


8.4.ANEXO IV - GUIA DE ENCAMINHAMENTOS PARA PESSOAS COM DEFICIÊNCIA E MOBILIDADE REDUZIDA

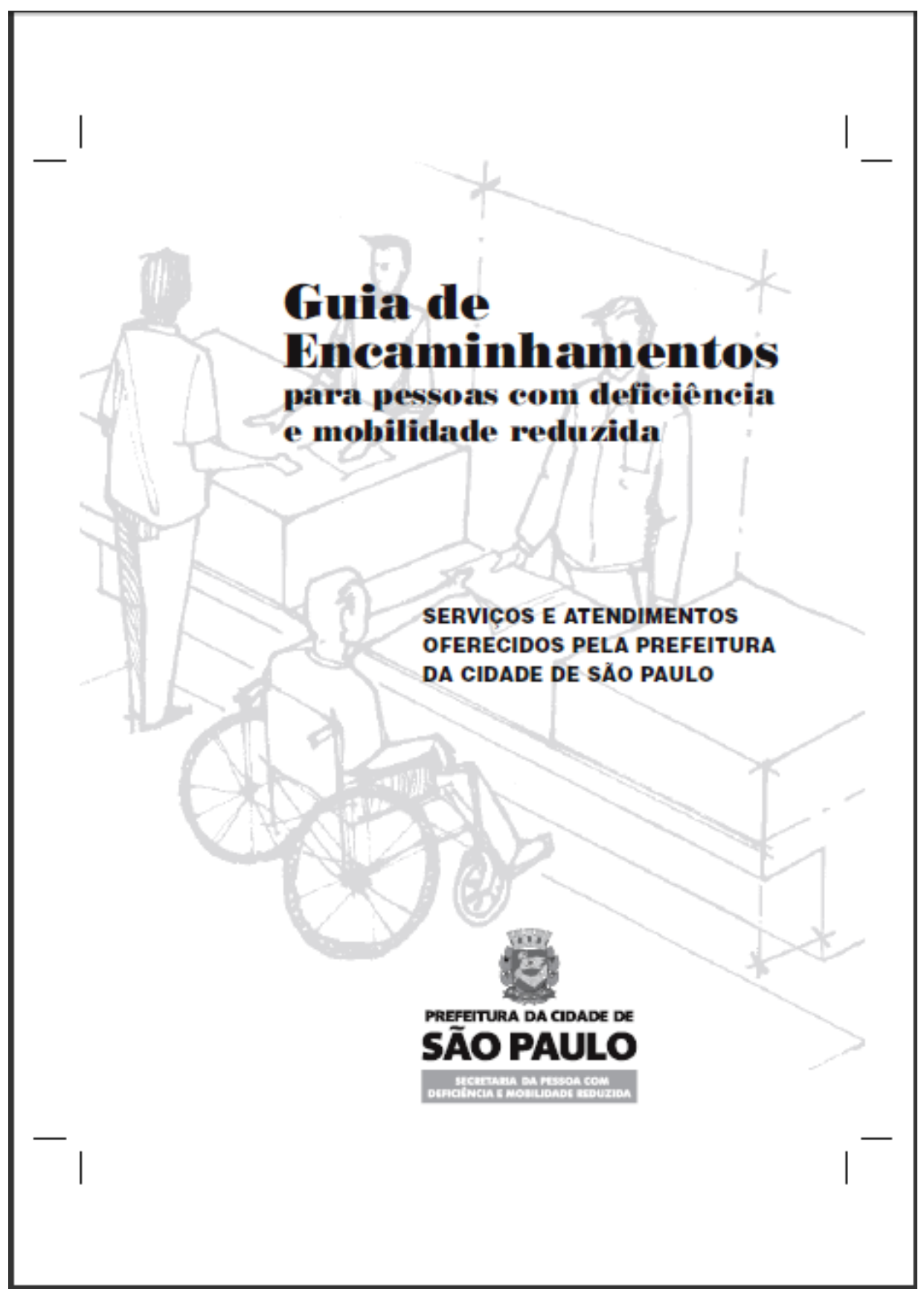




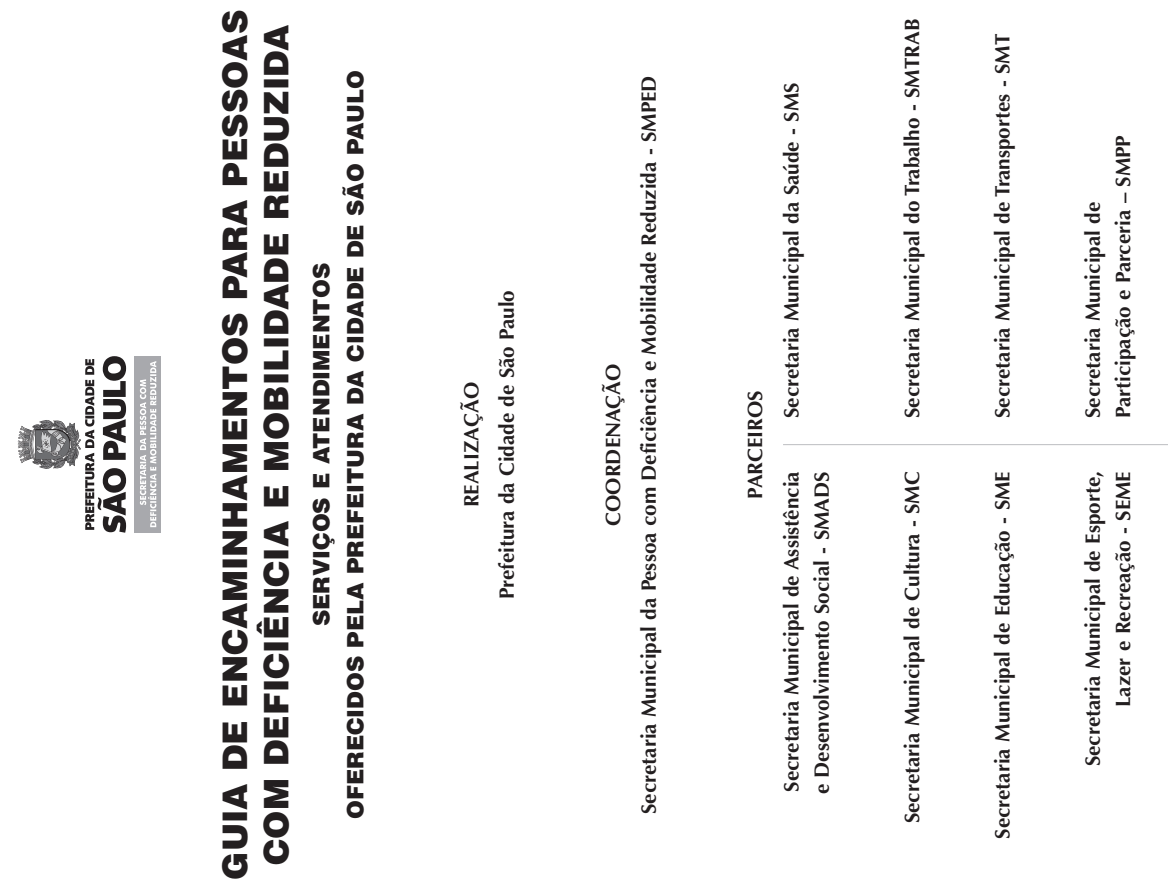



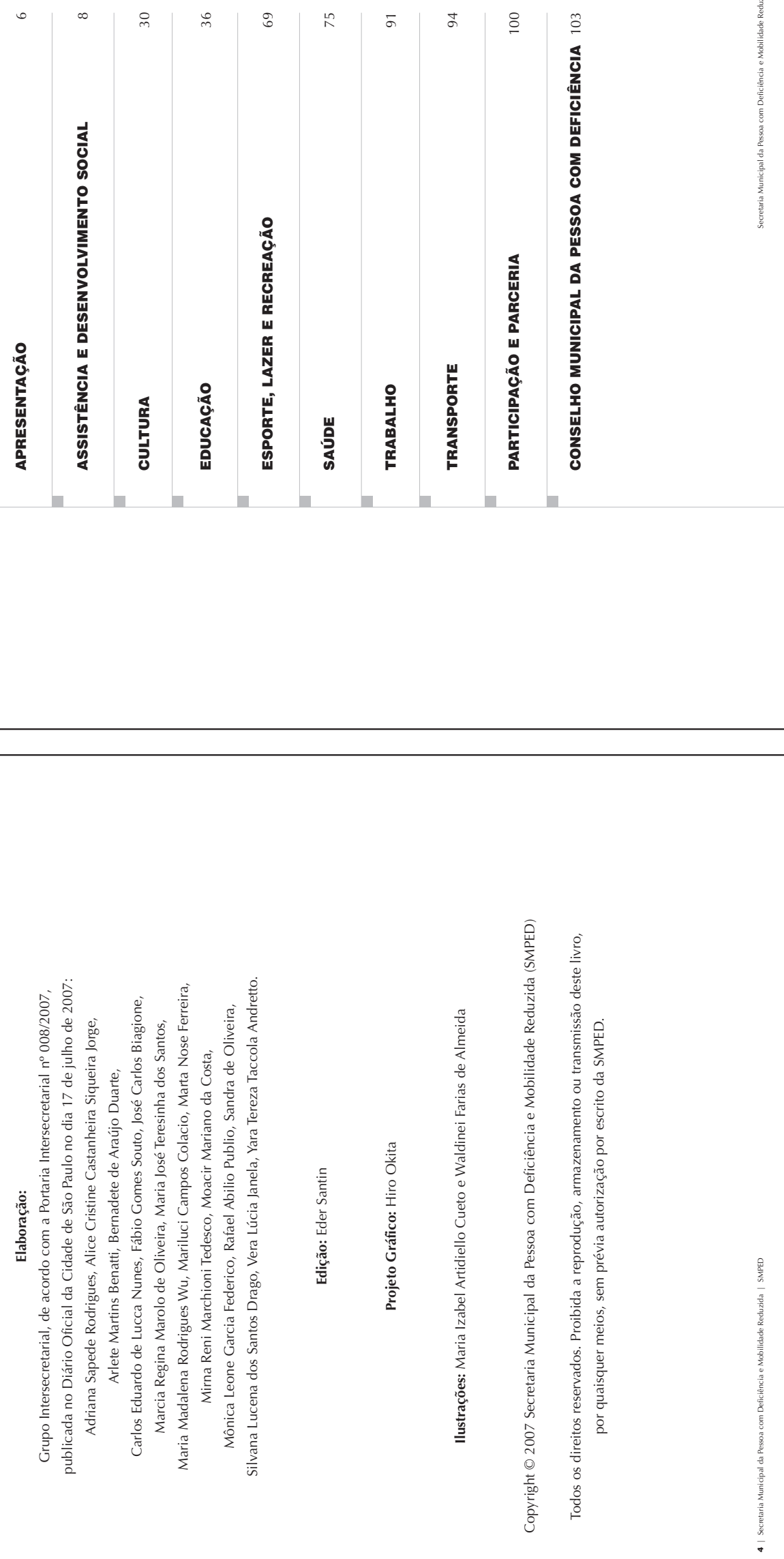

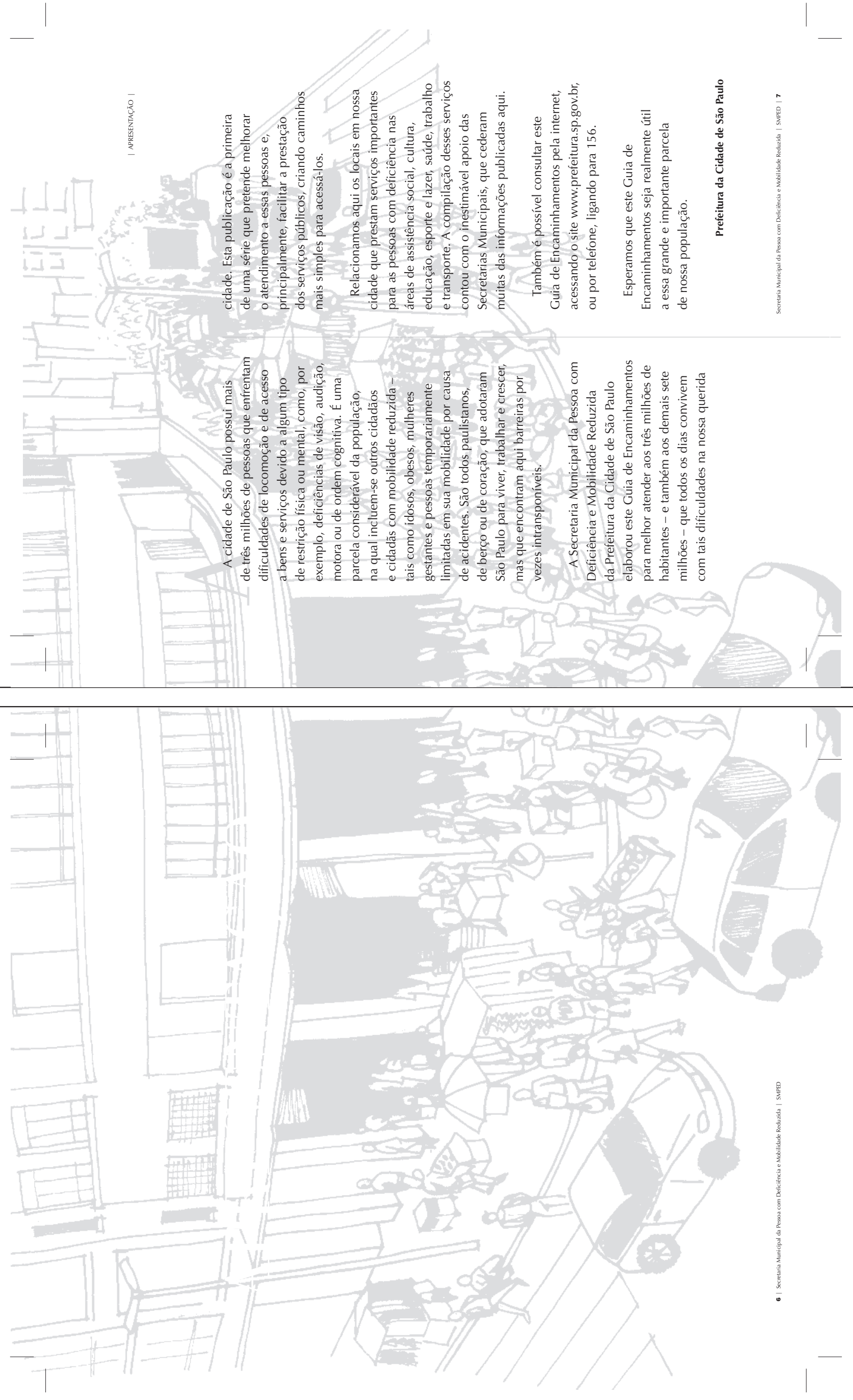

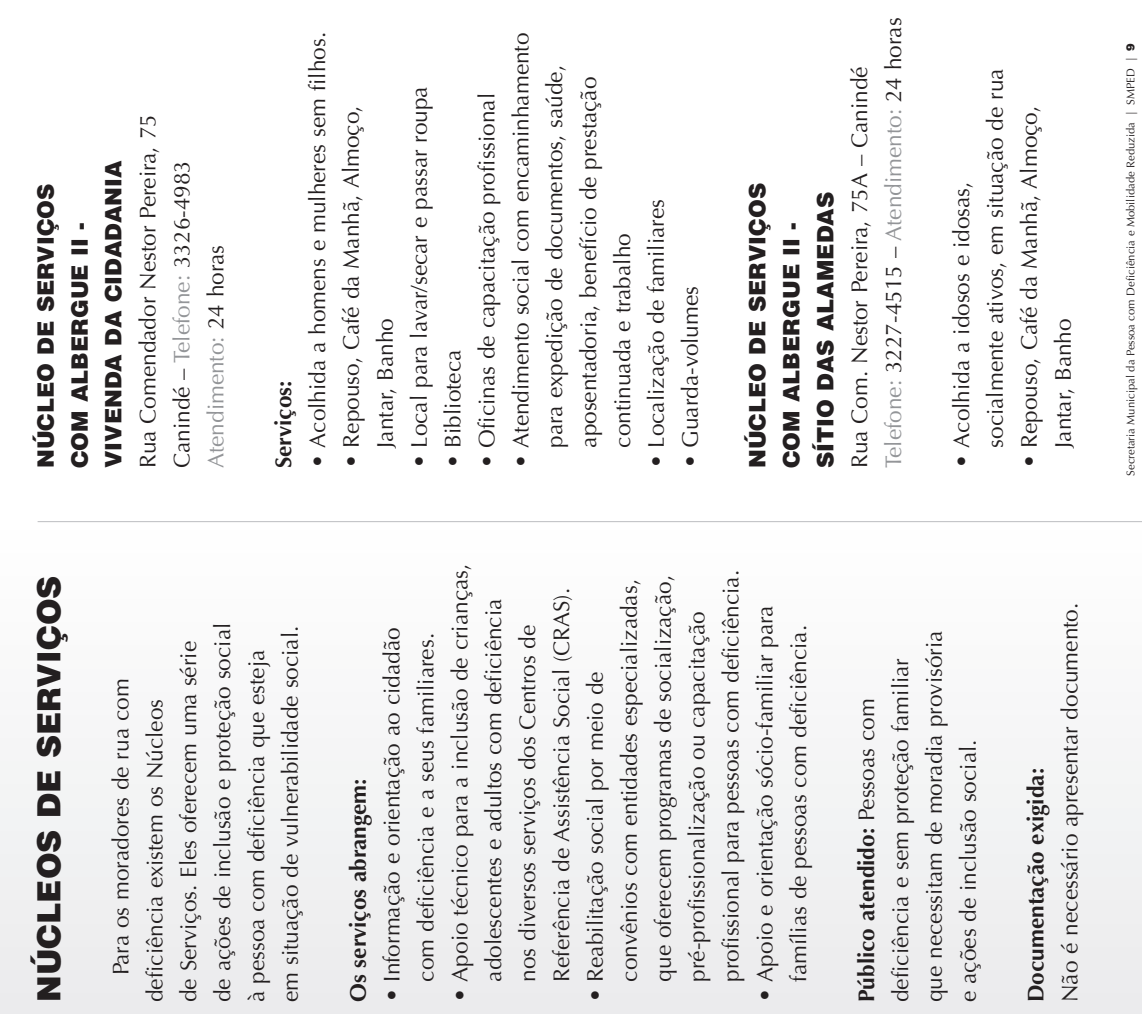

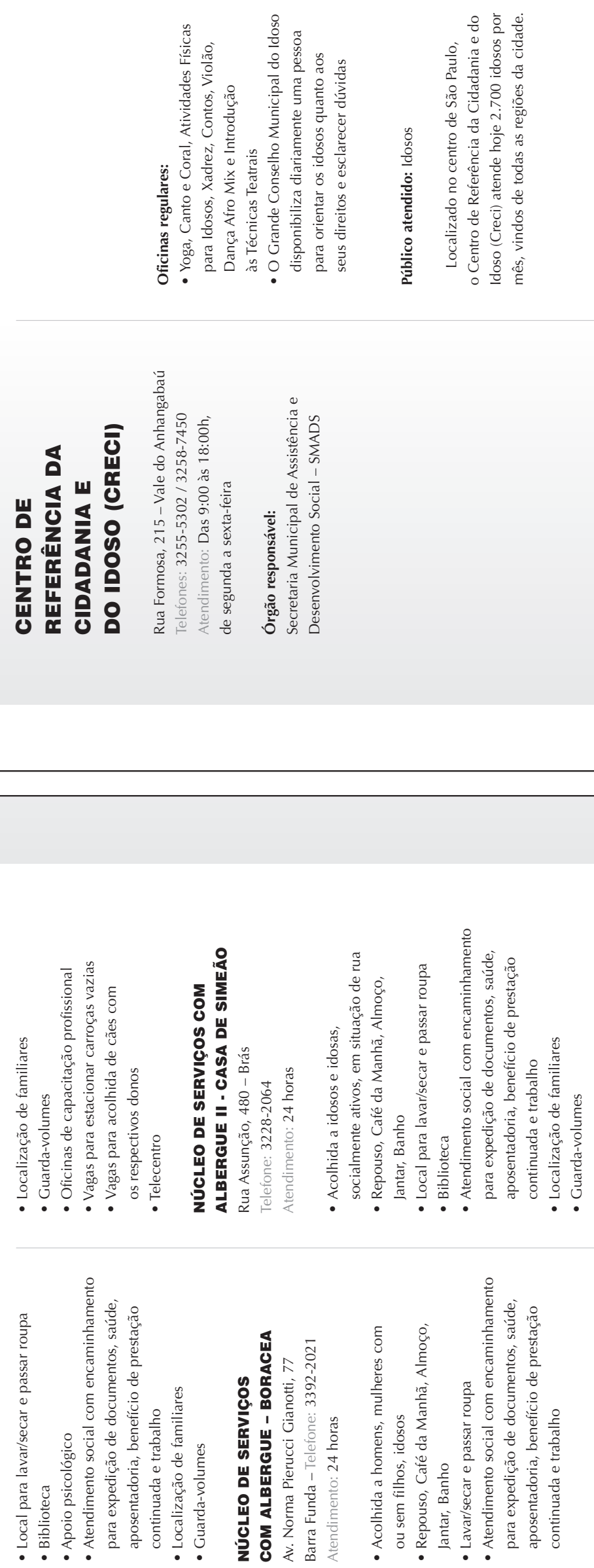

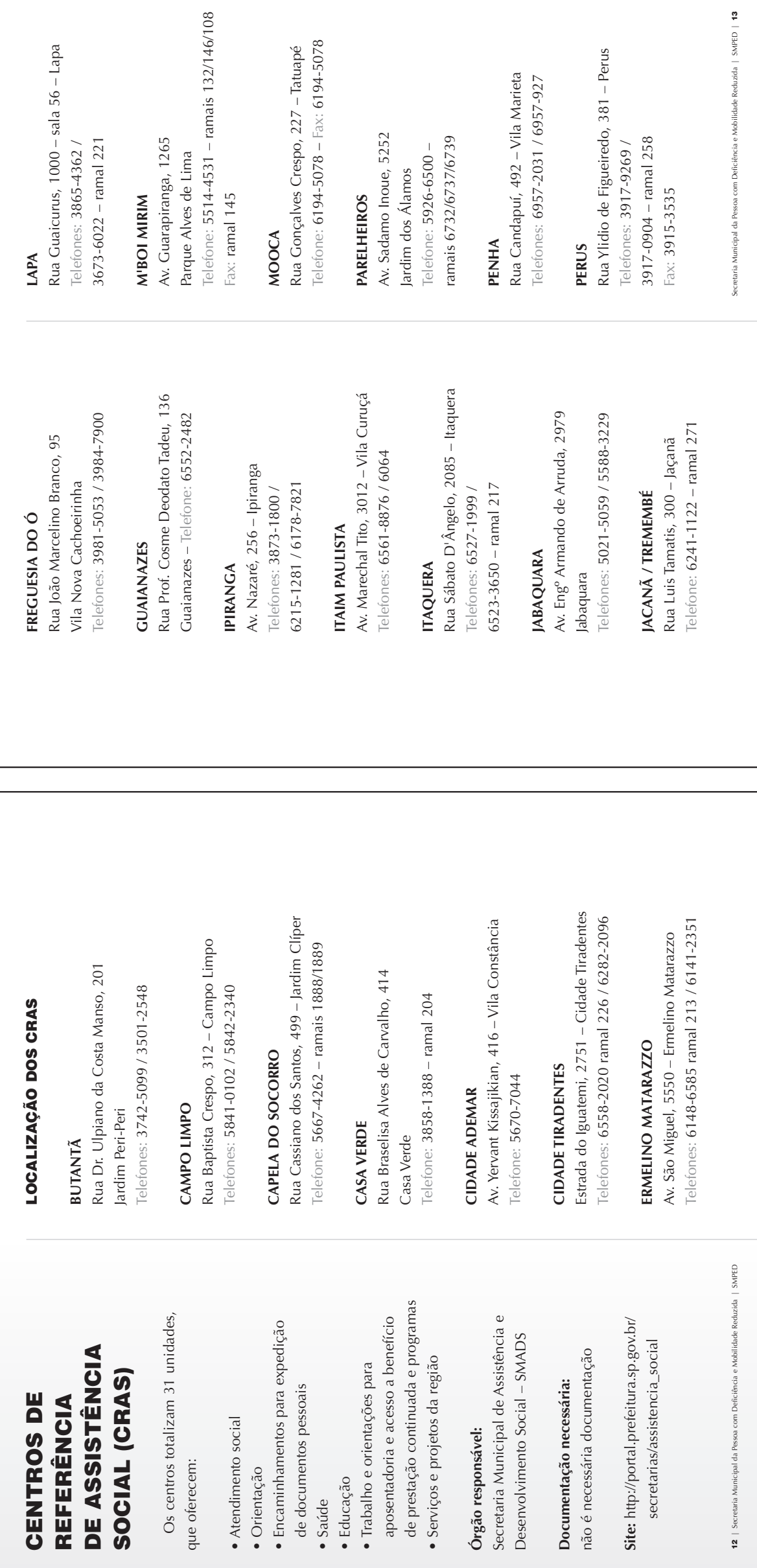

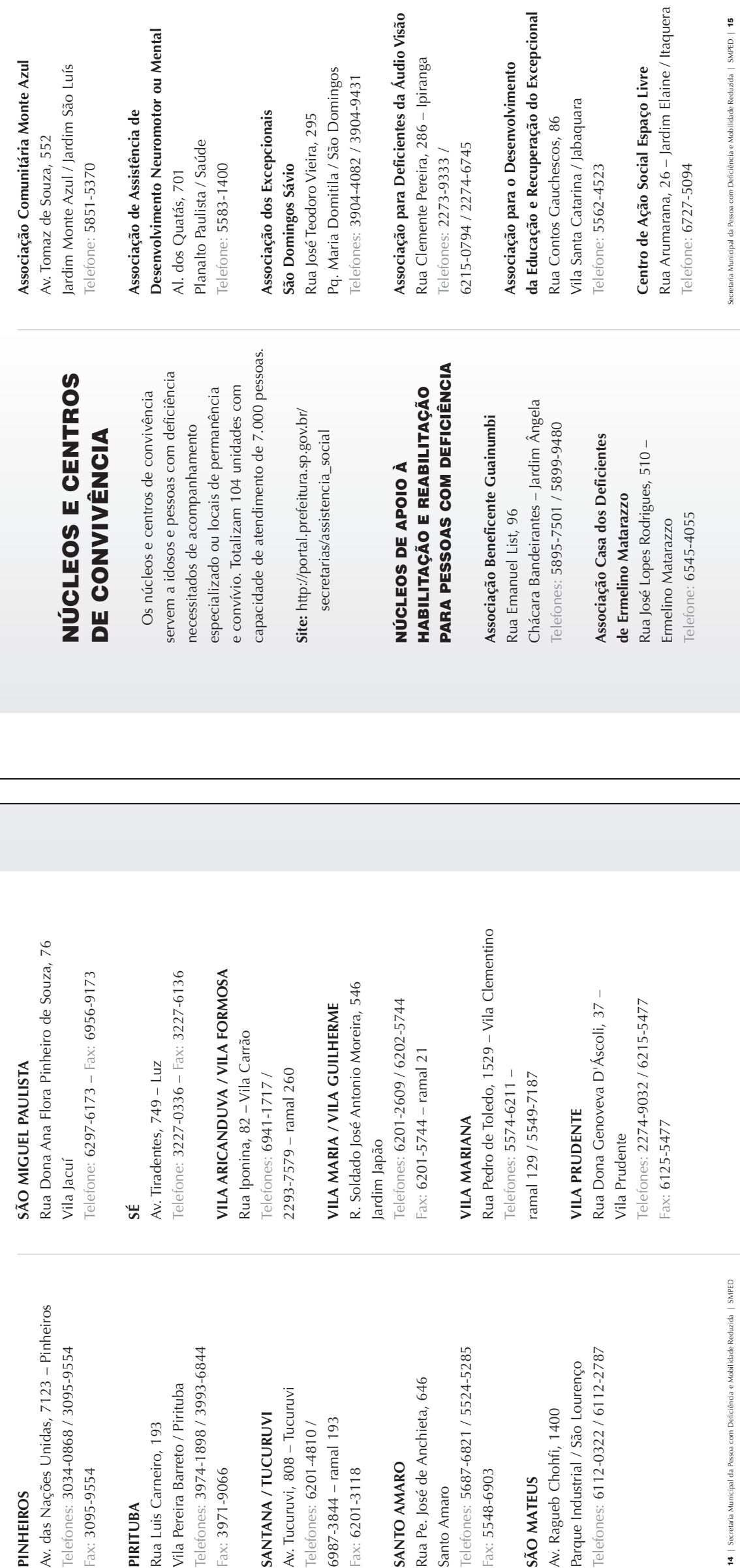


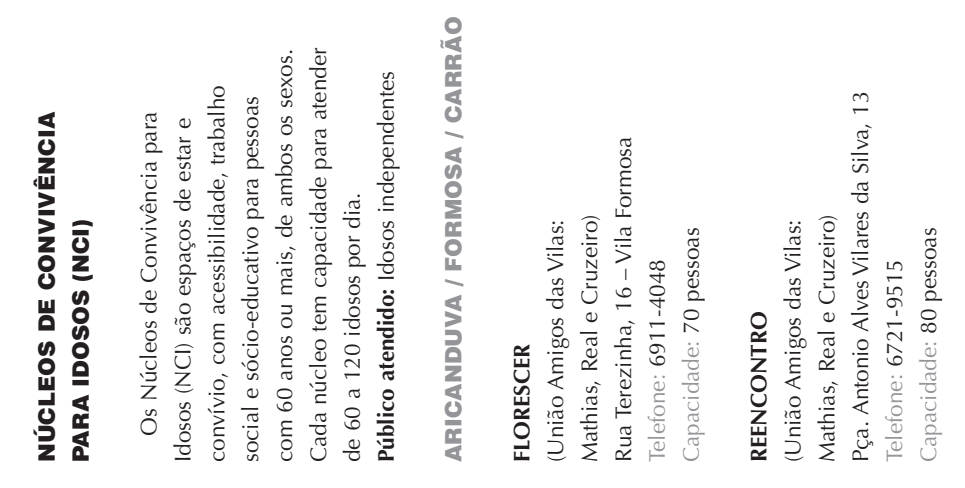

12

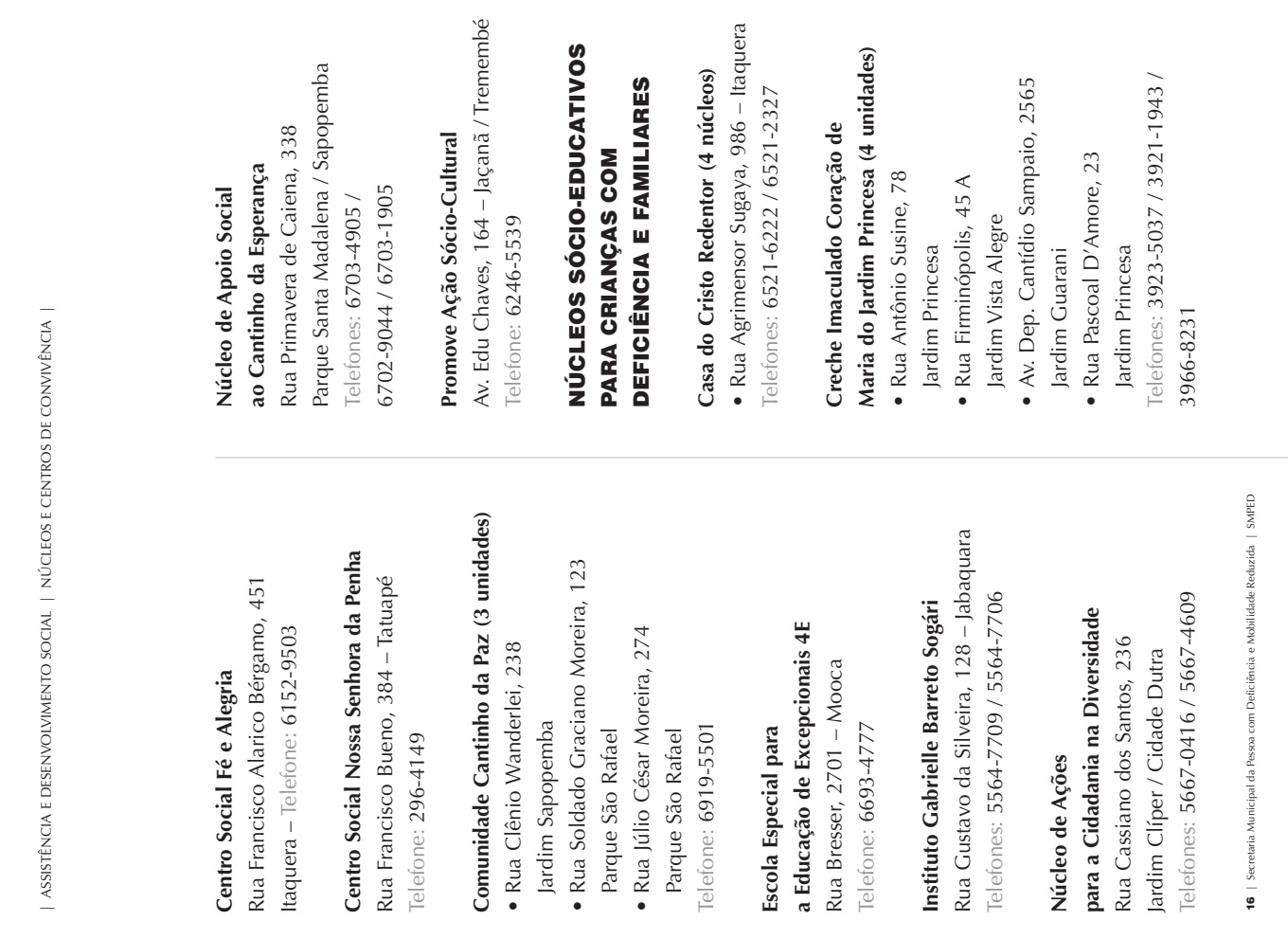



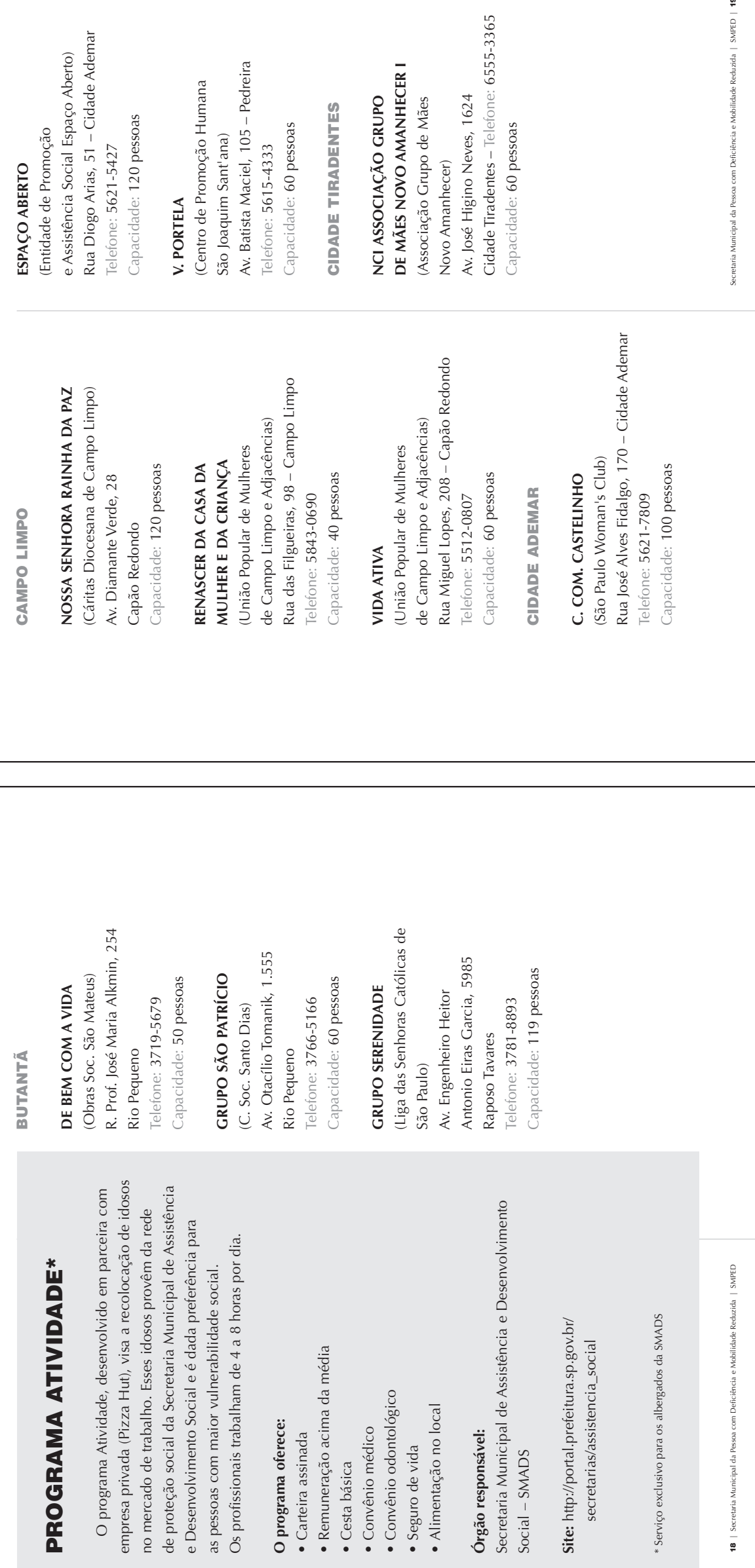

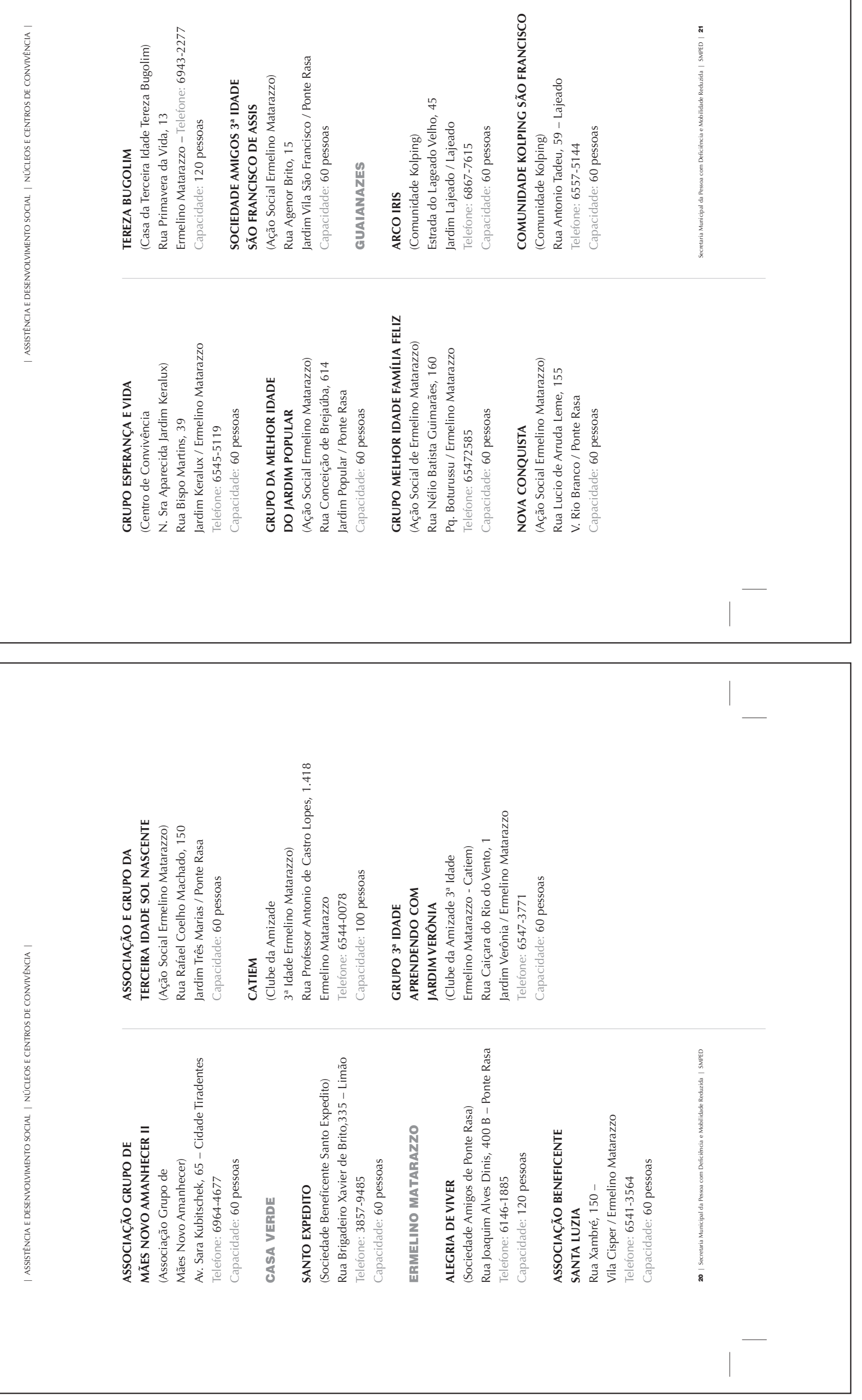

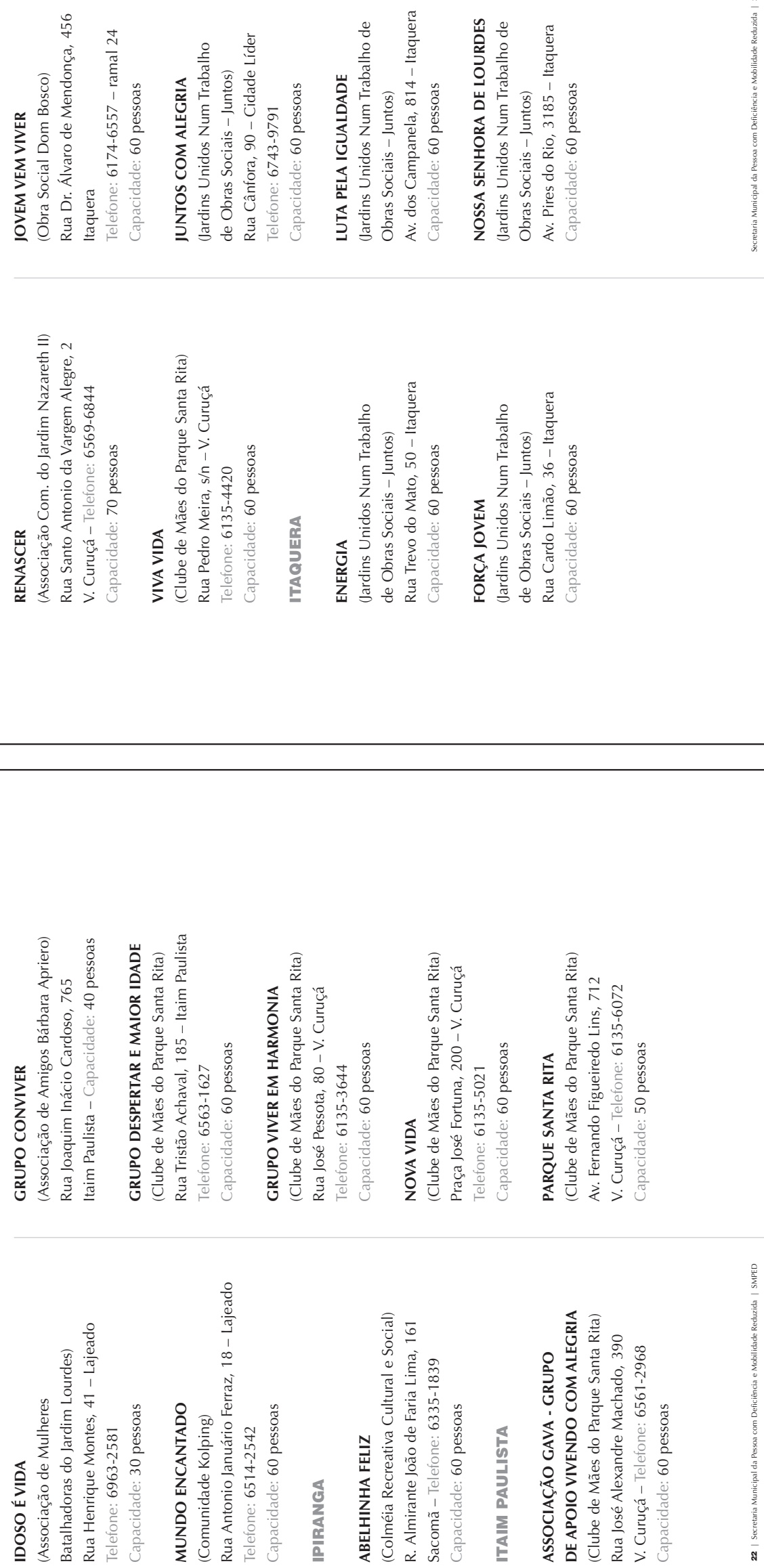

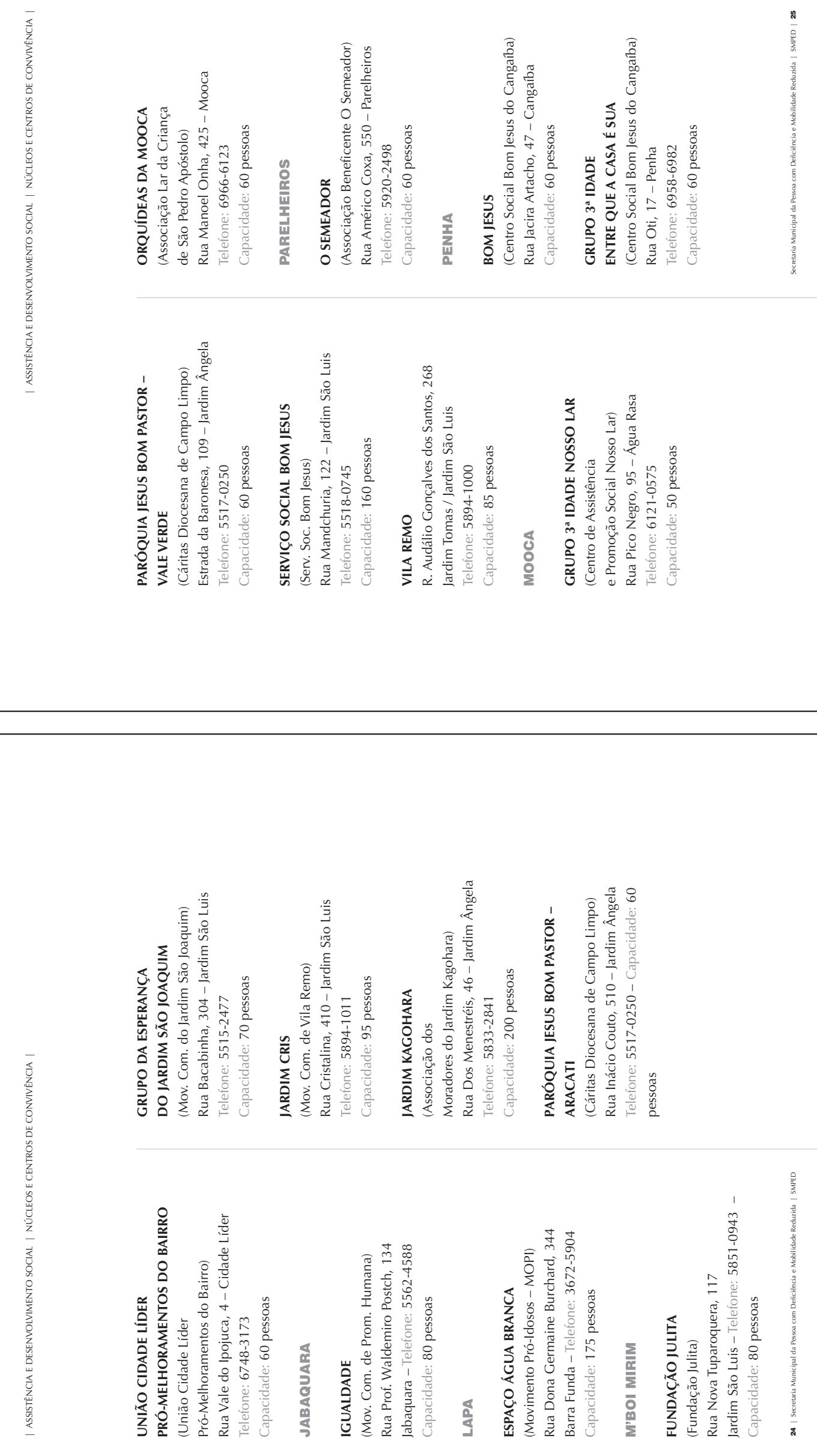

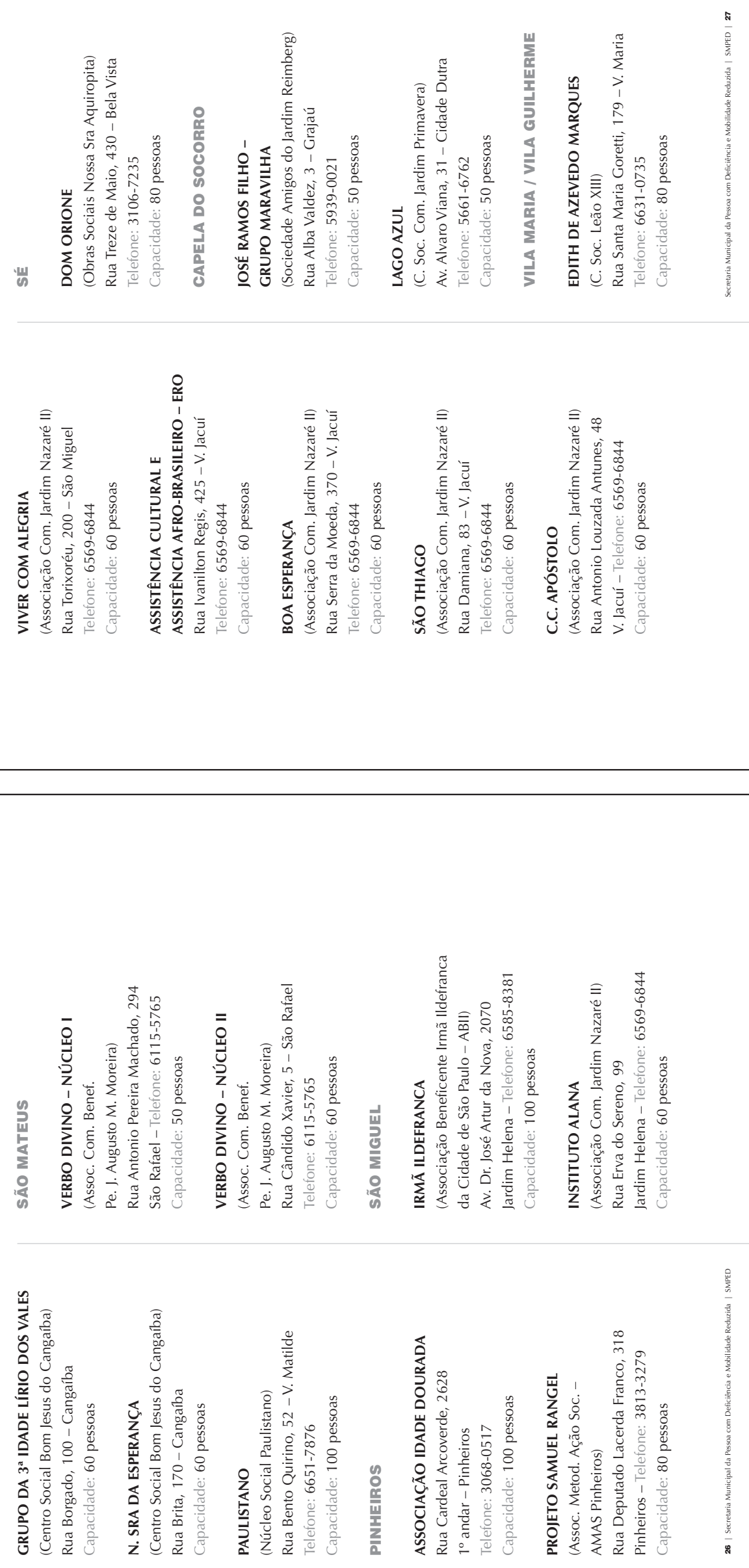

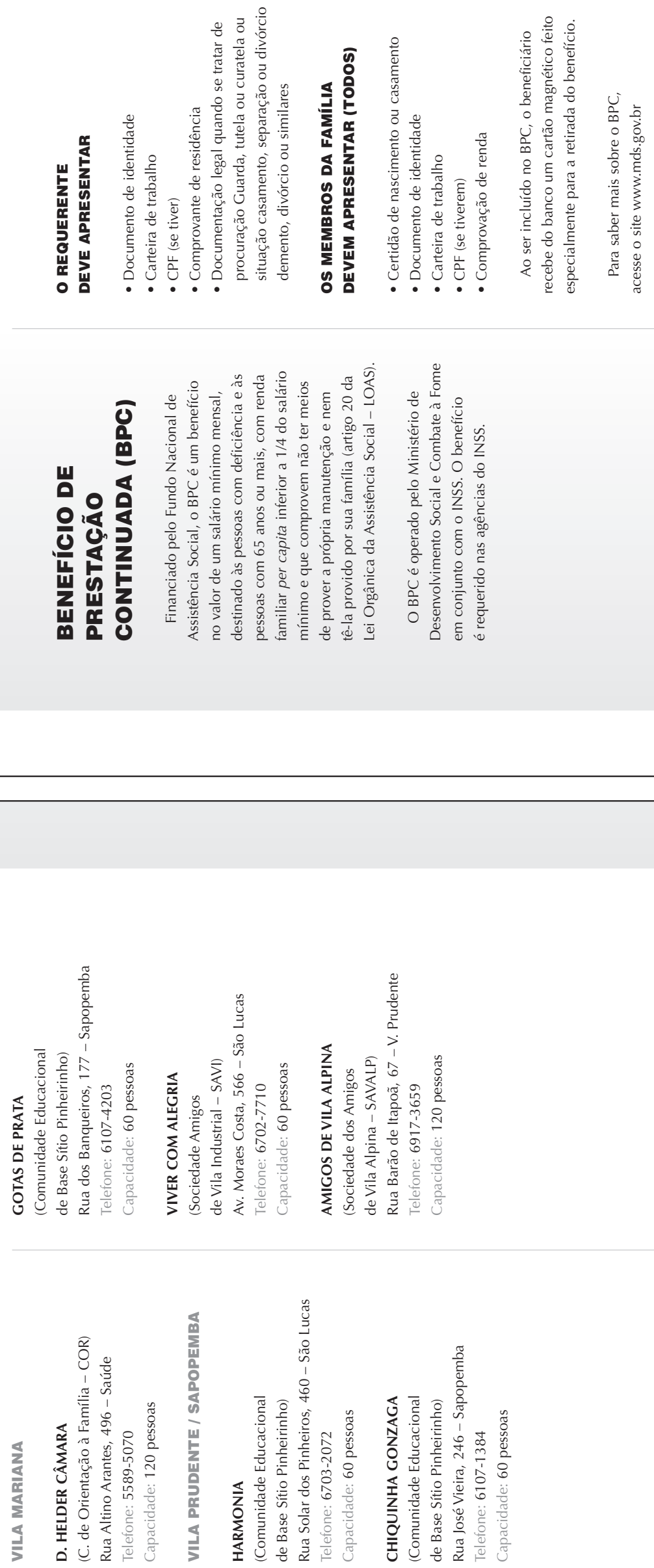

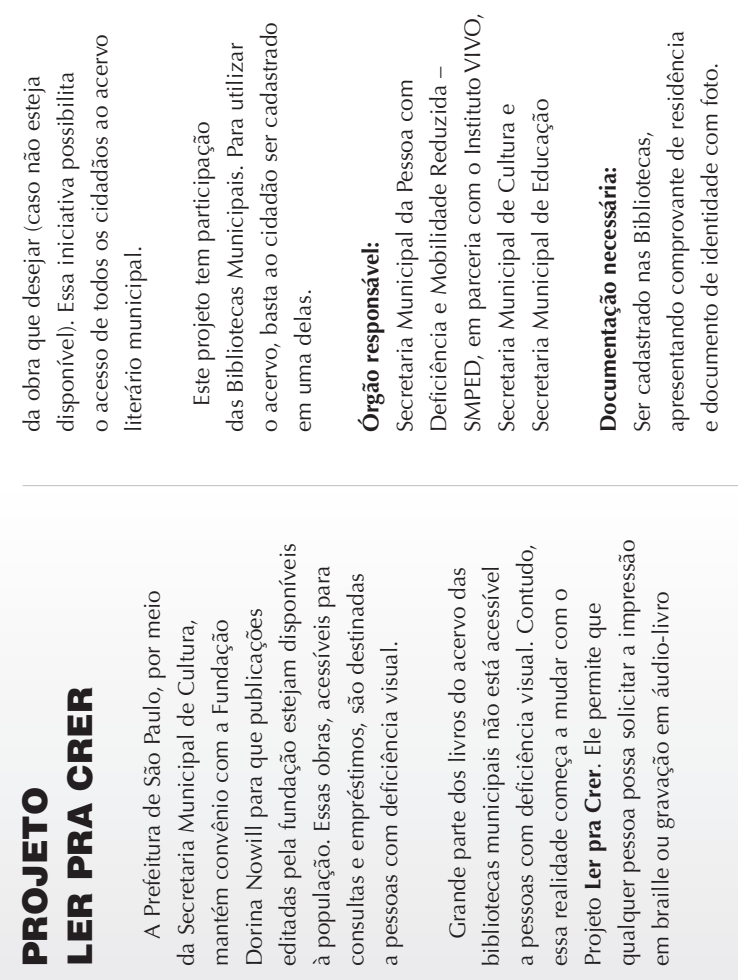

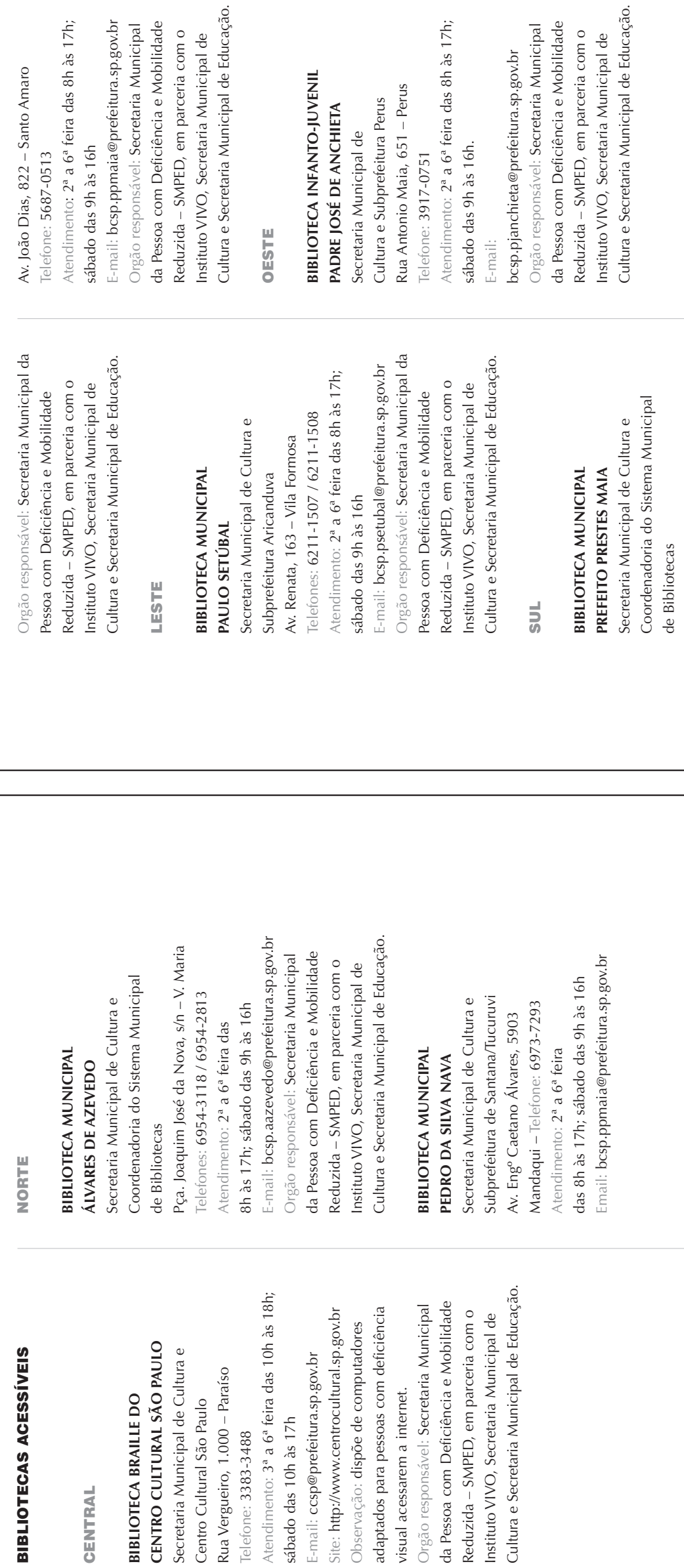

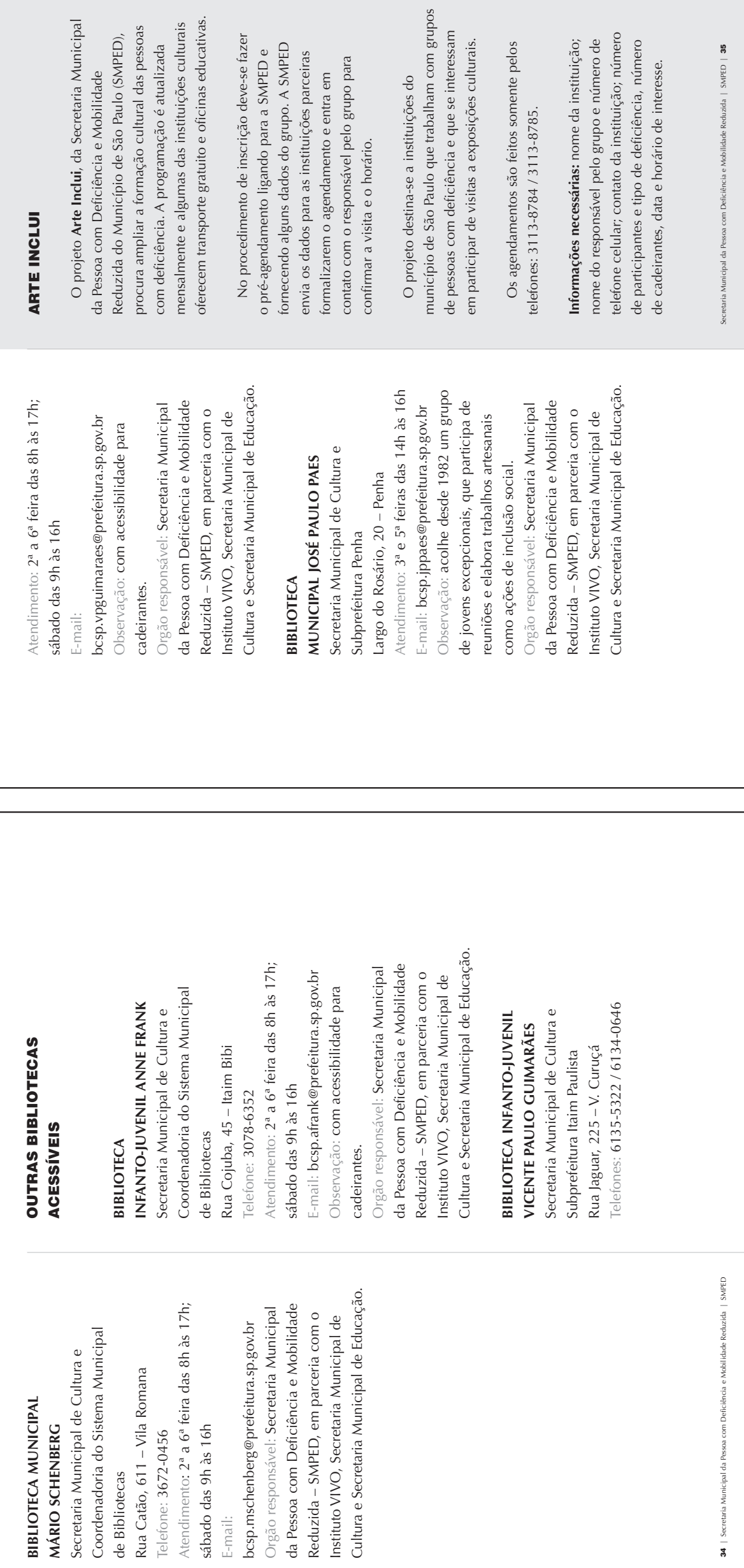

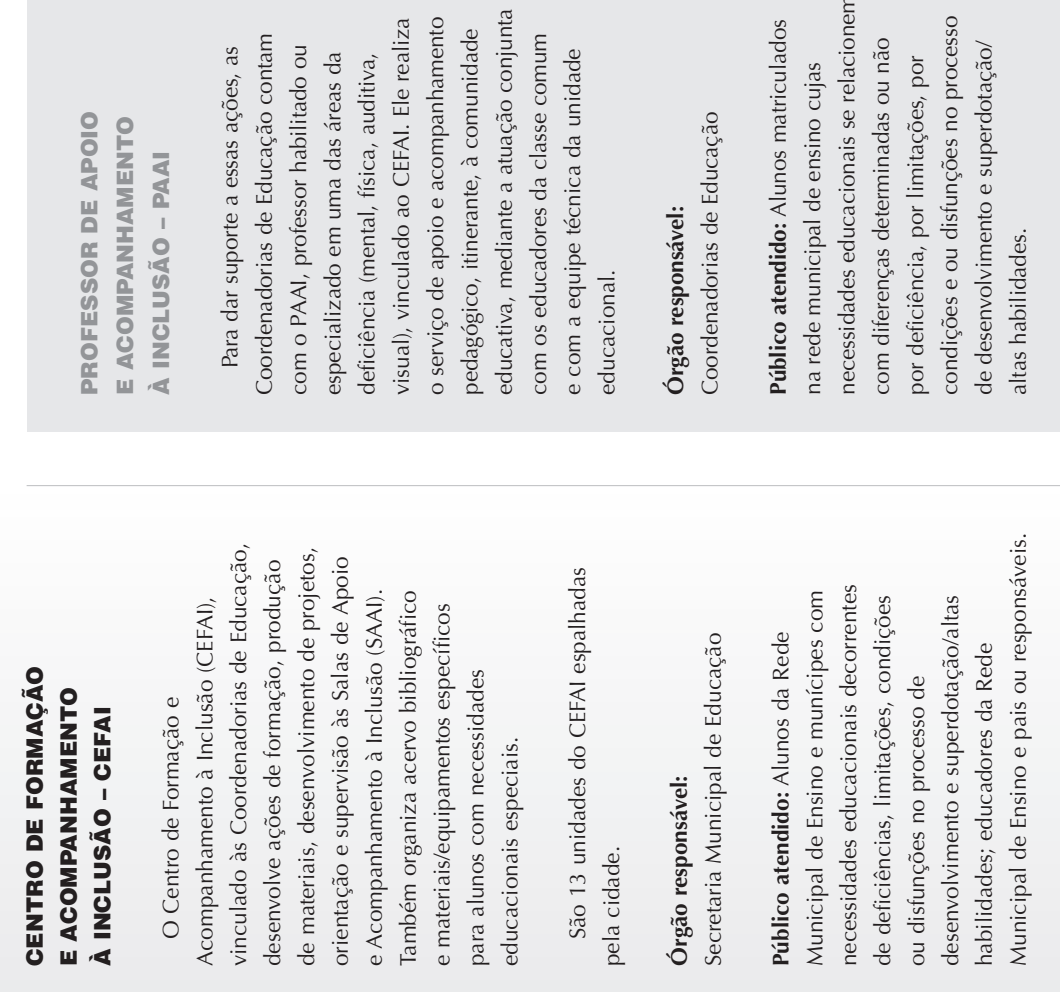

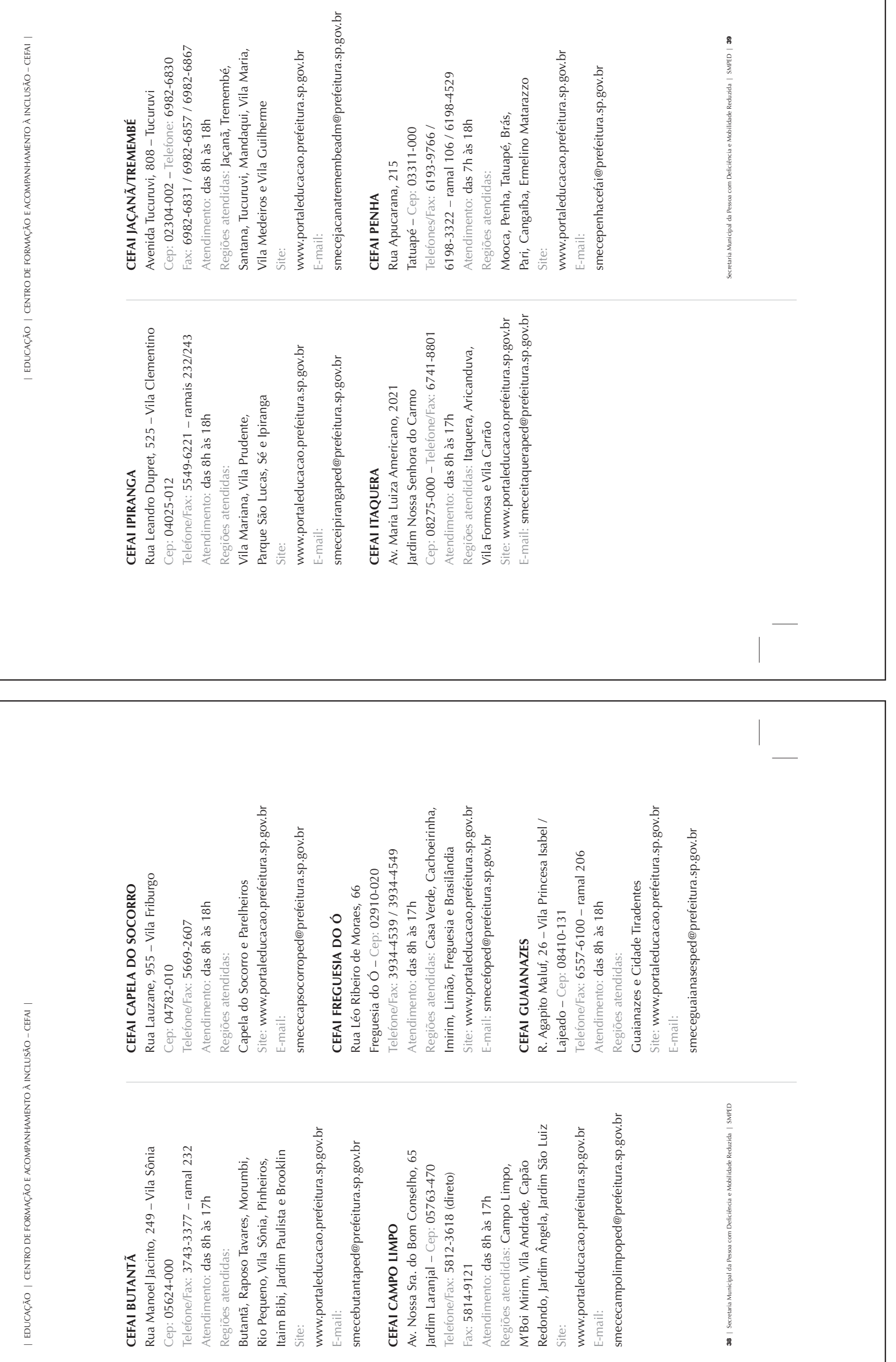

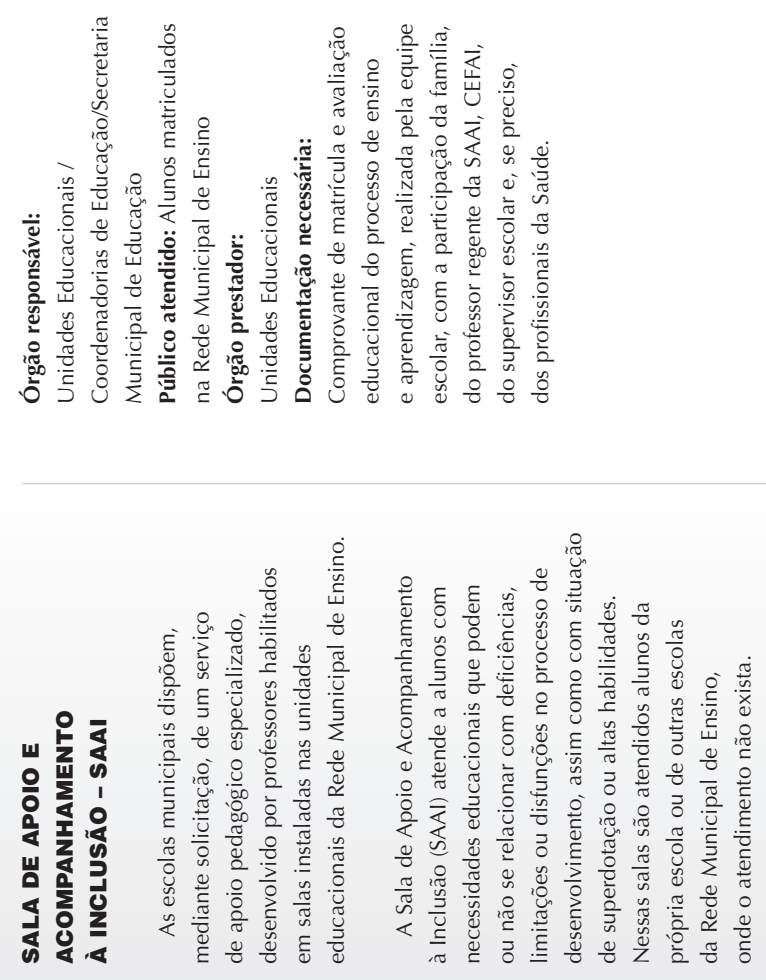

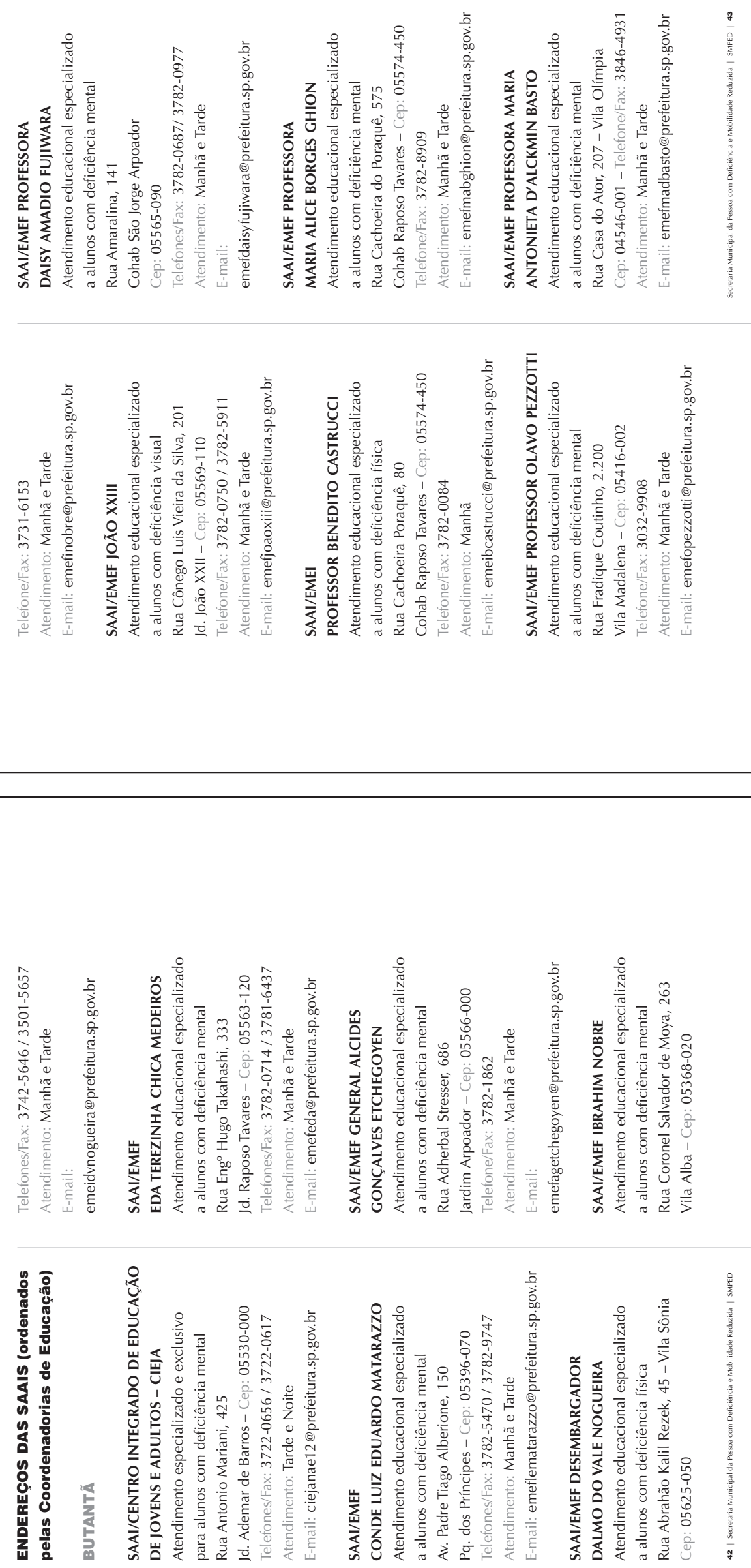

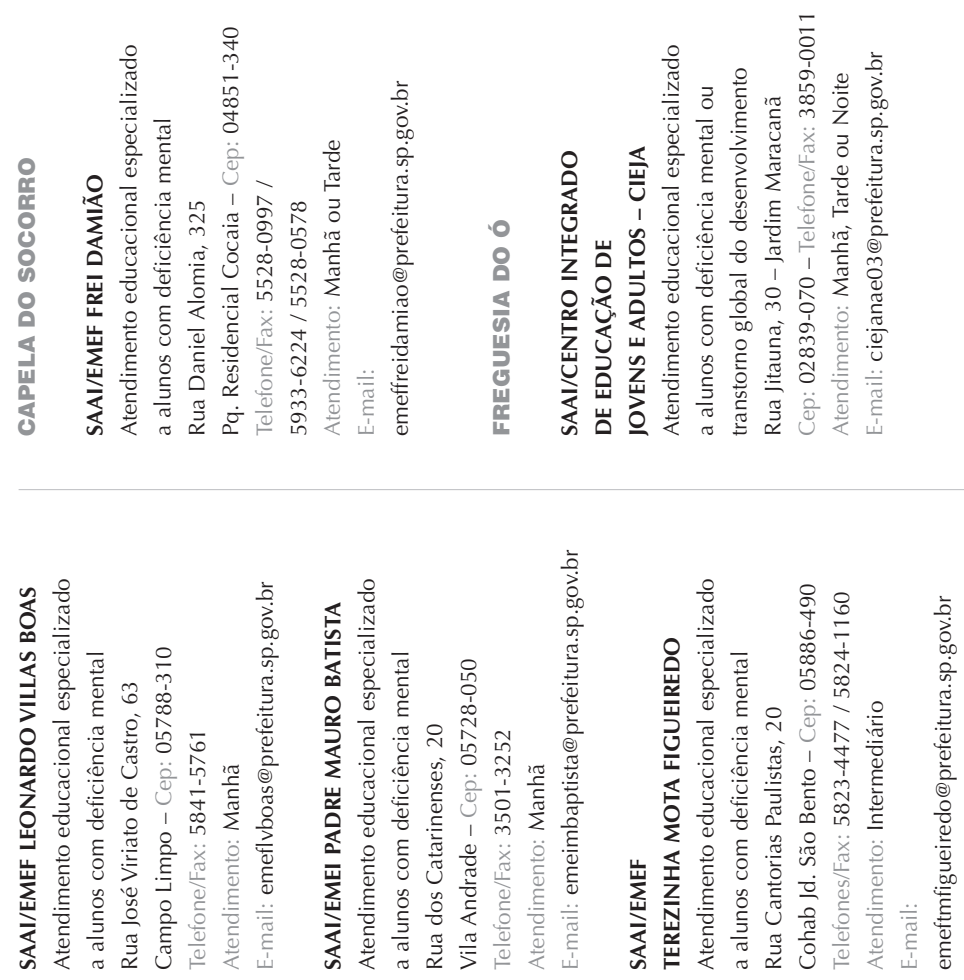


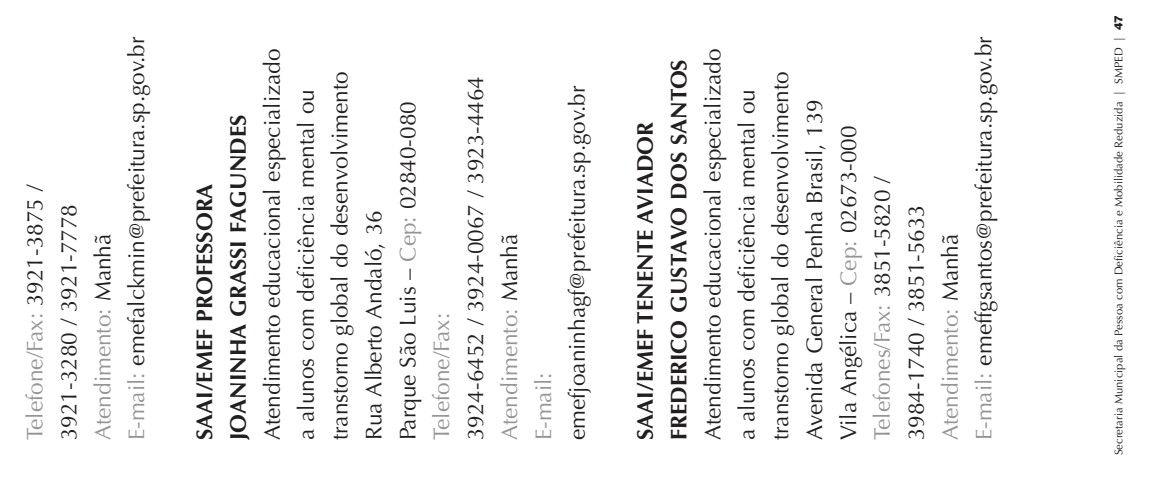

MU⿴囗十) 

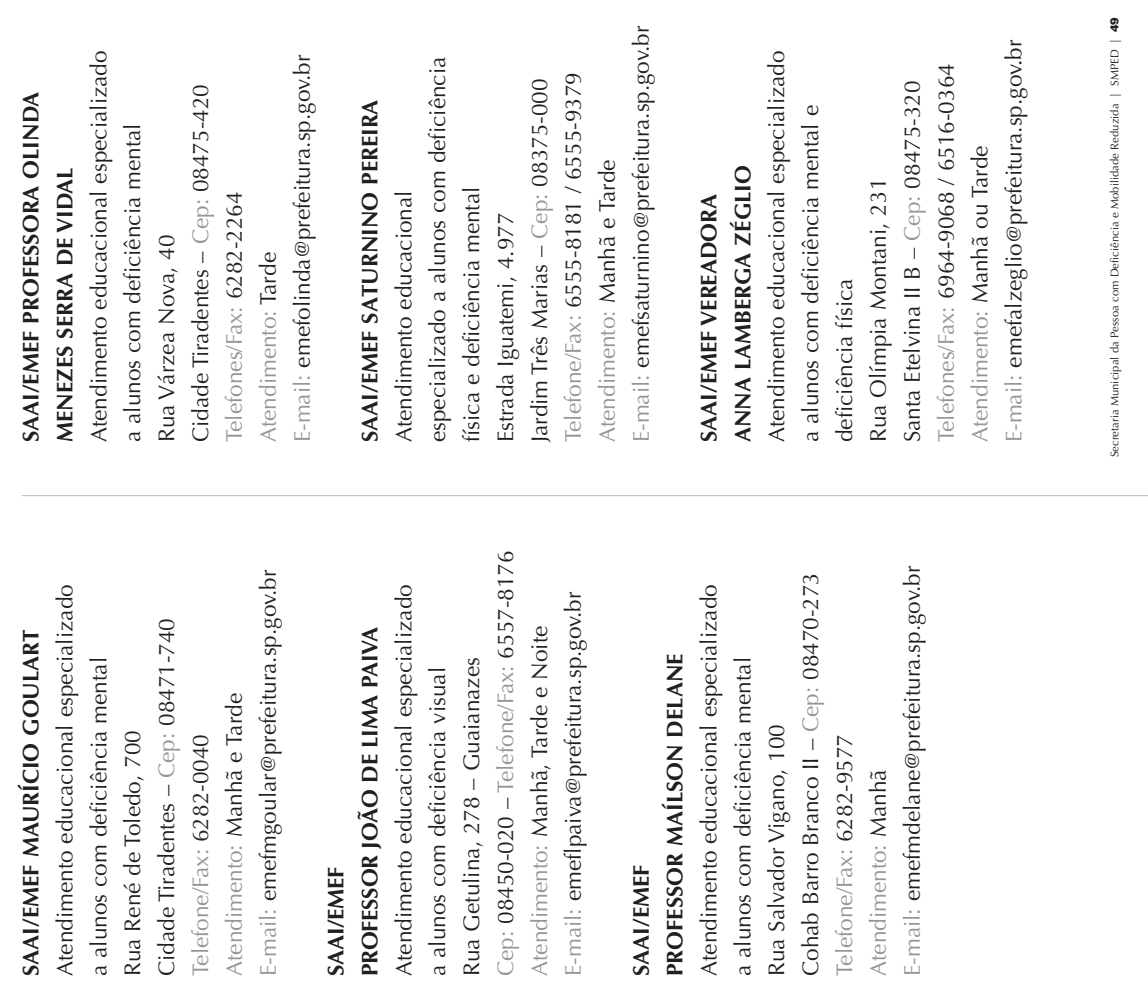

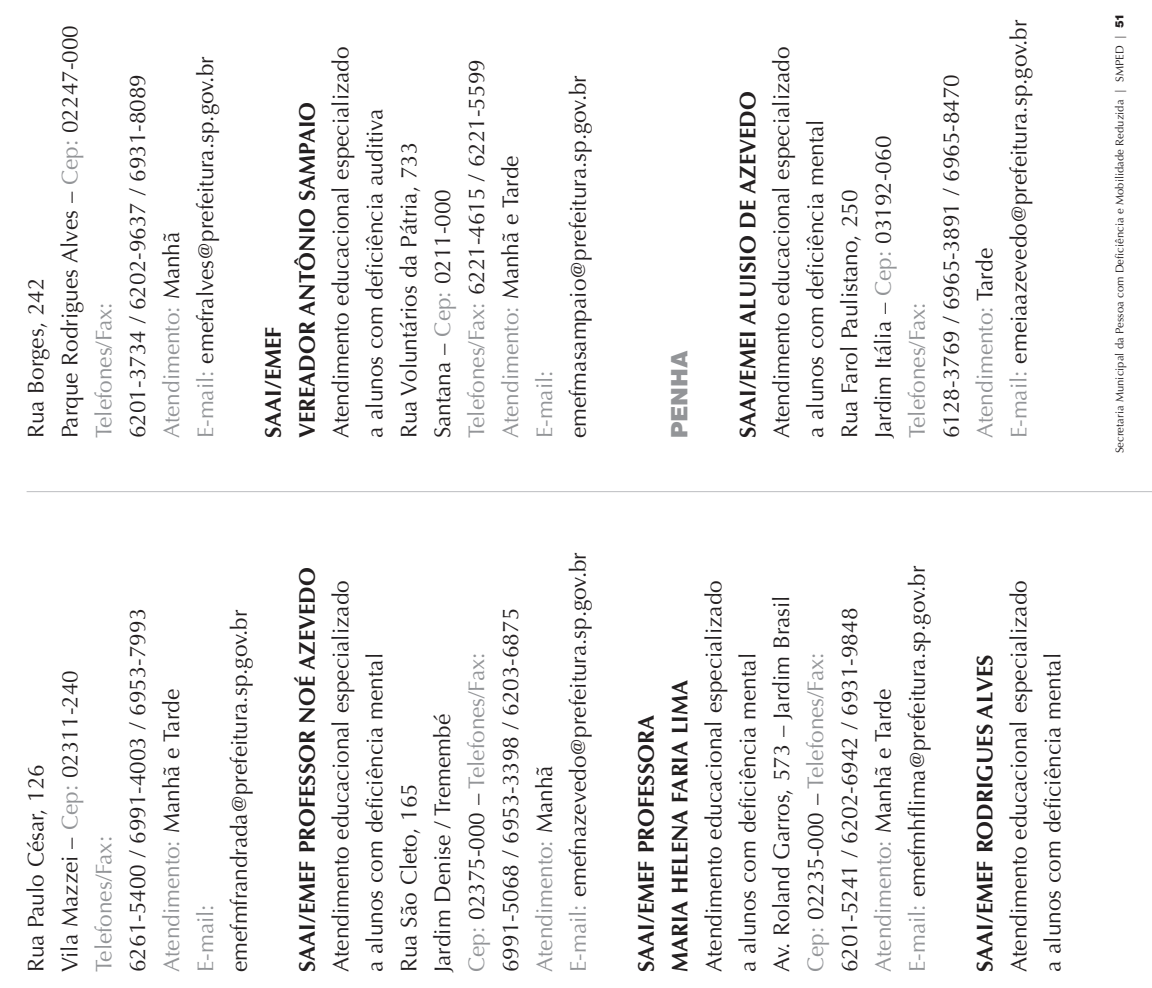

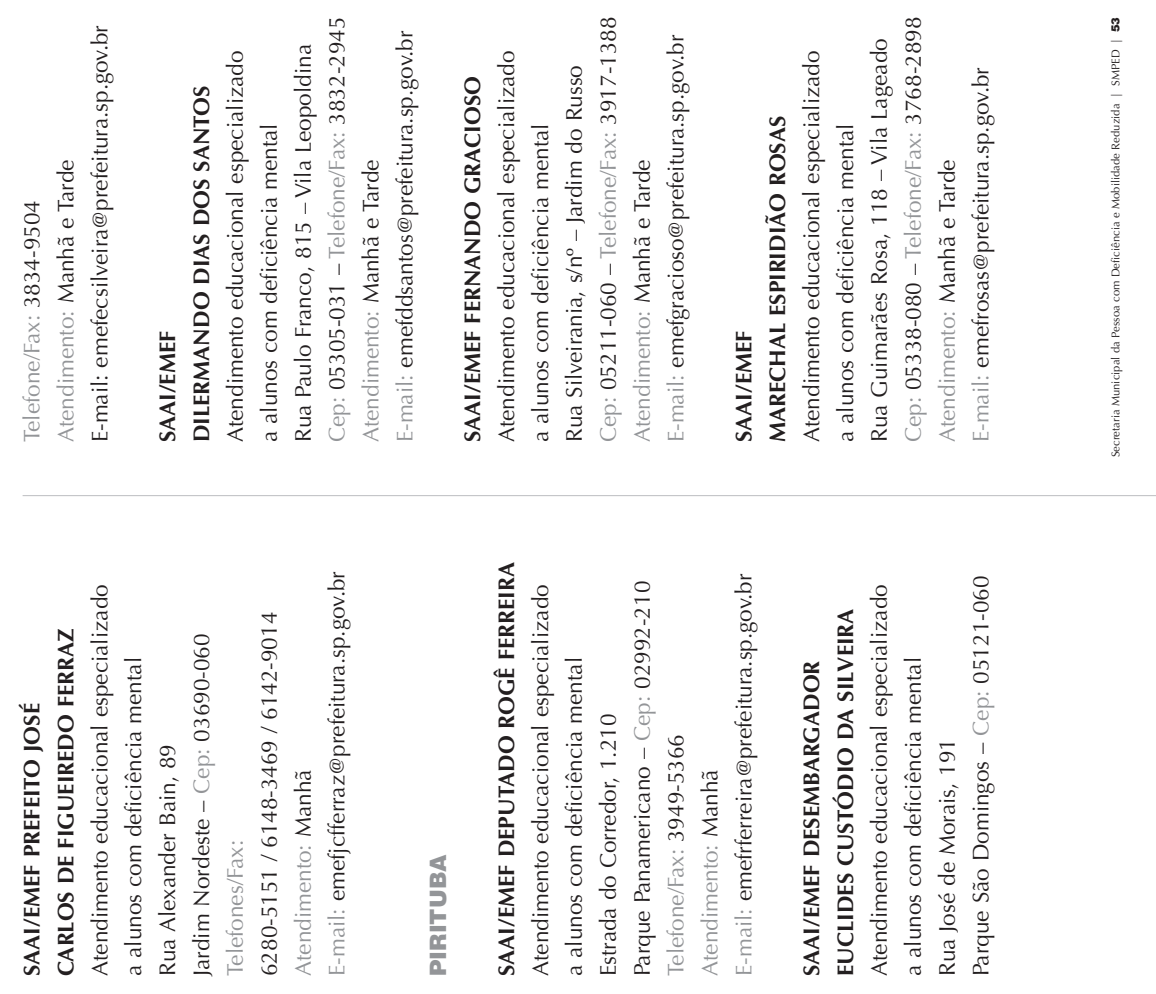


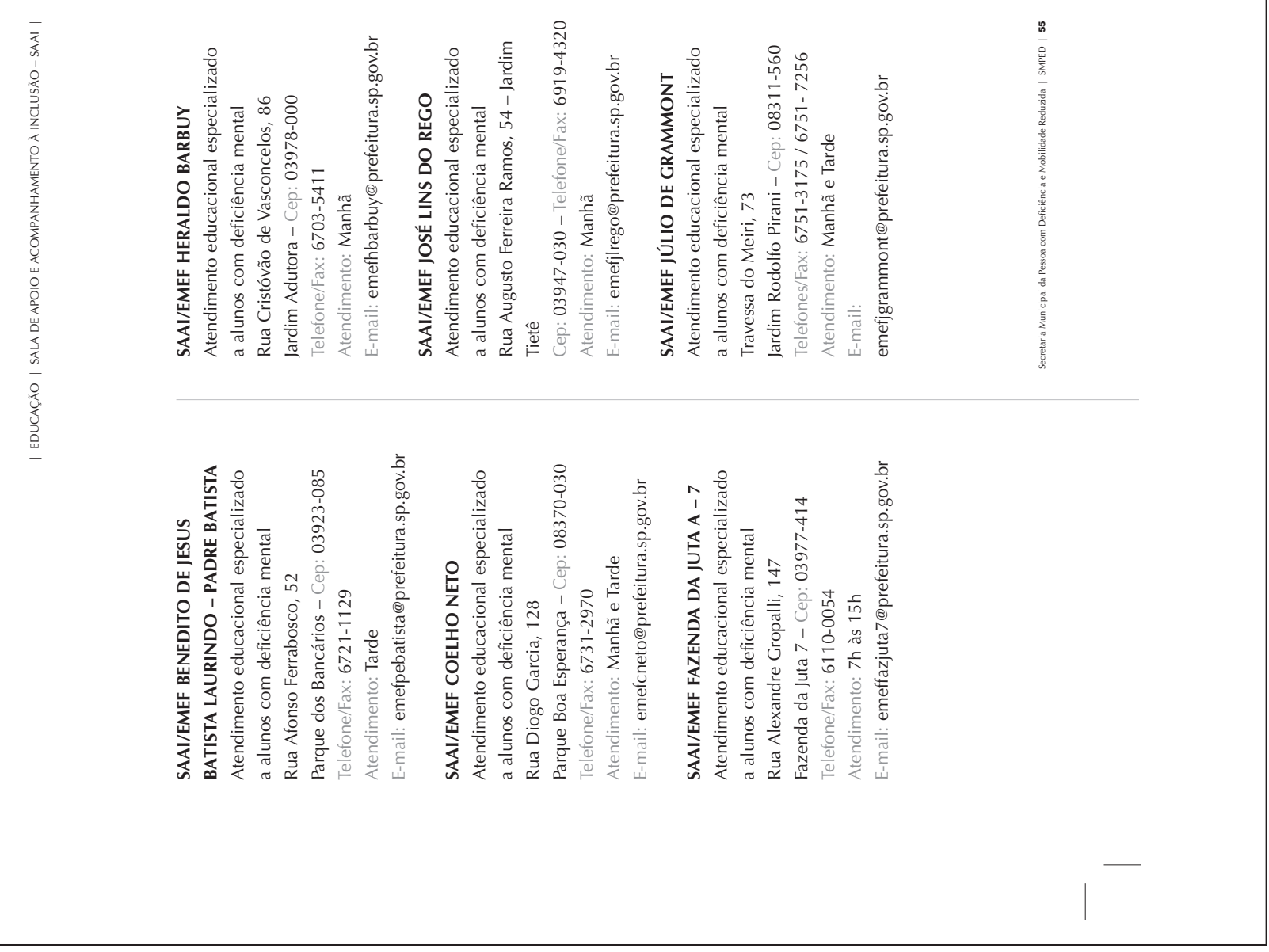

(2)
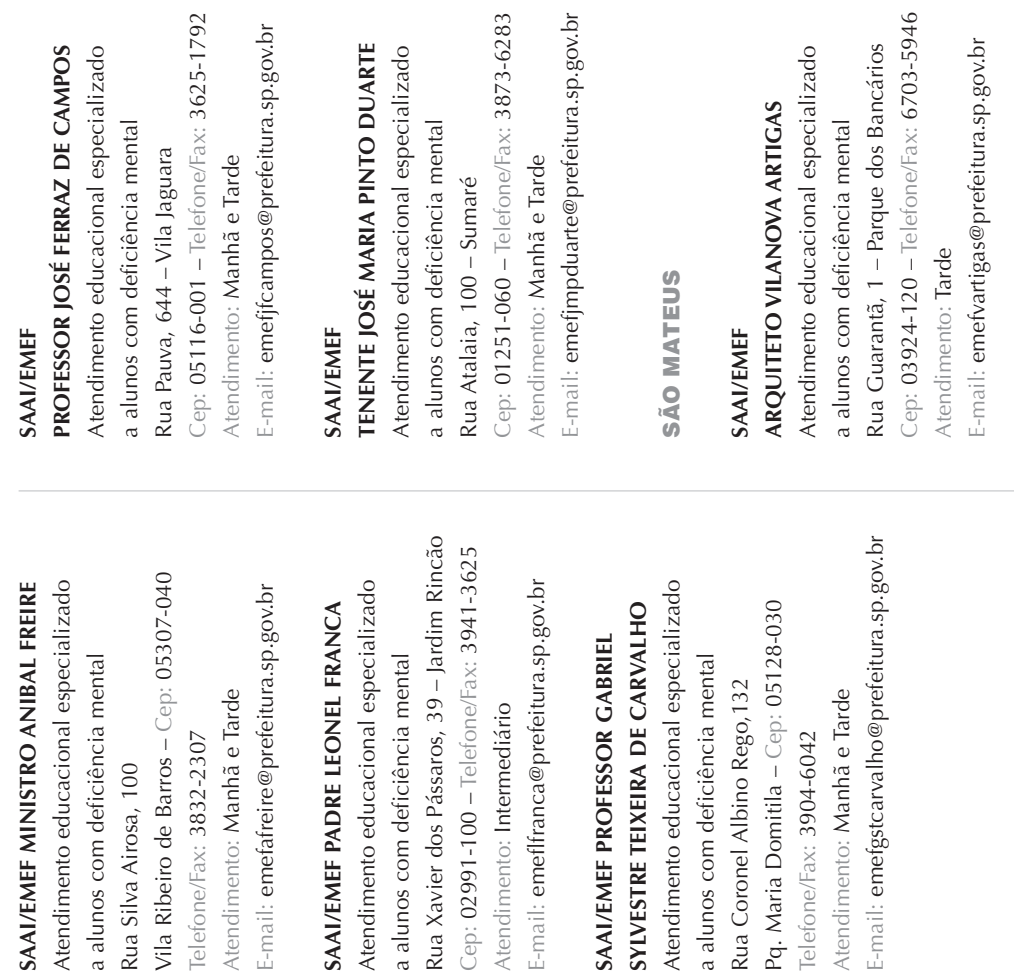

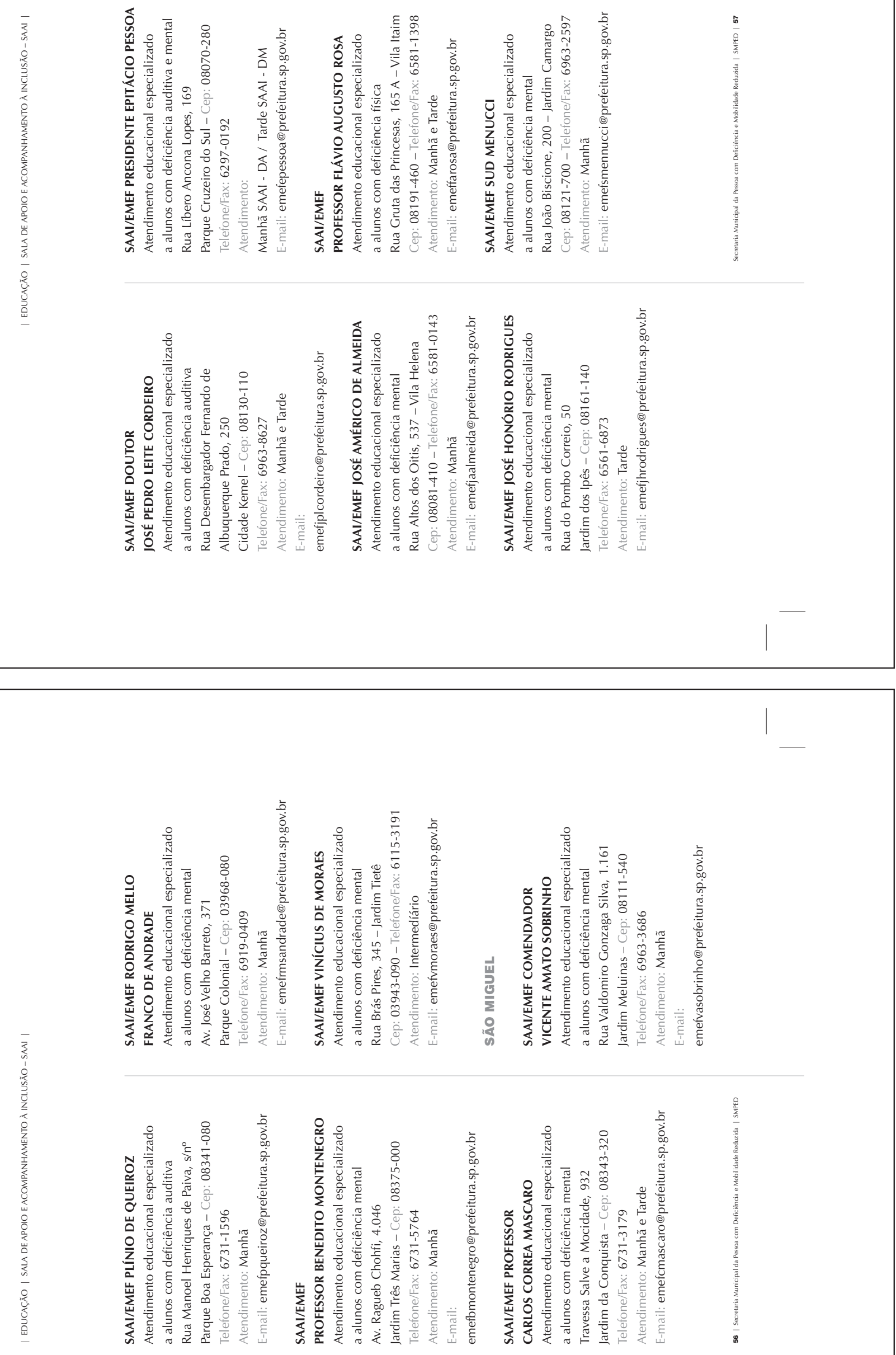

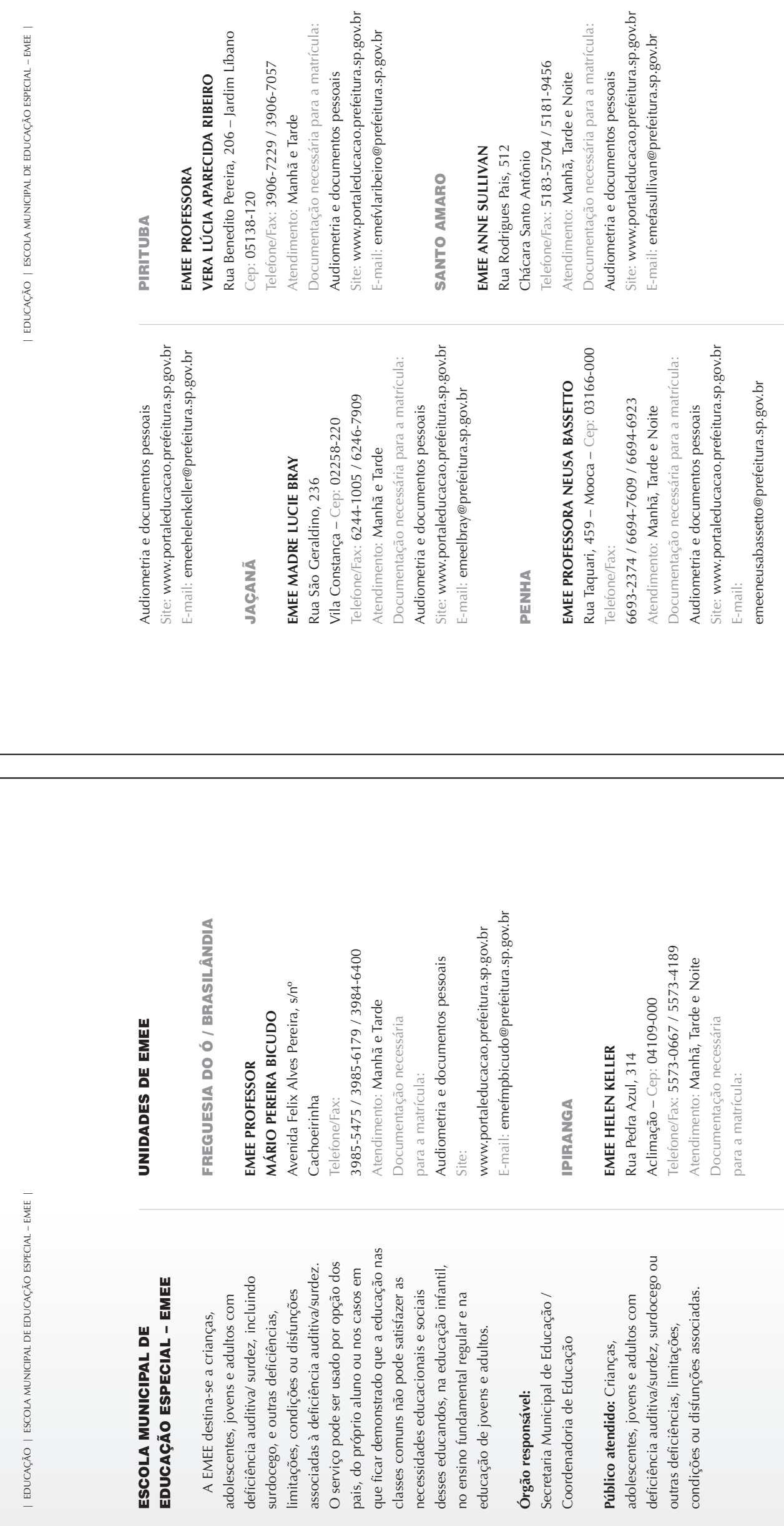

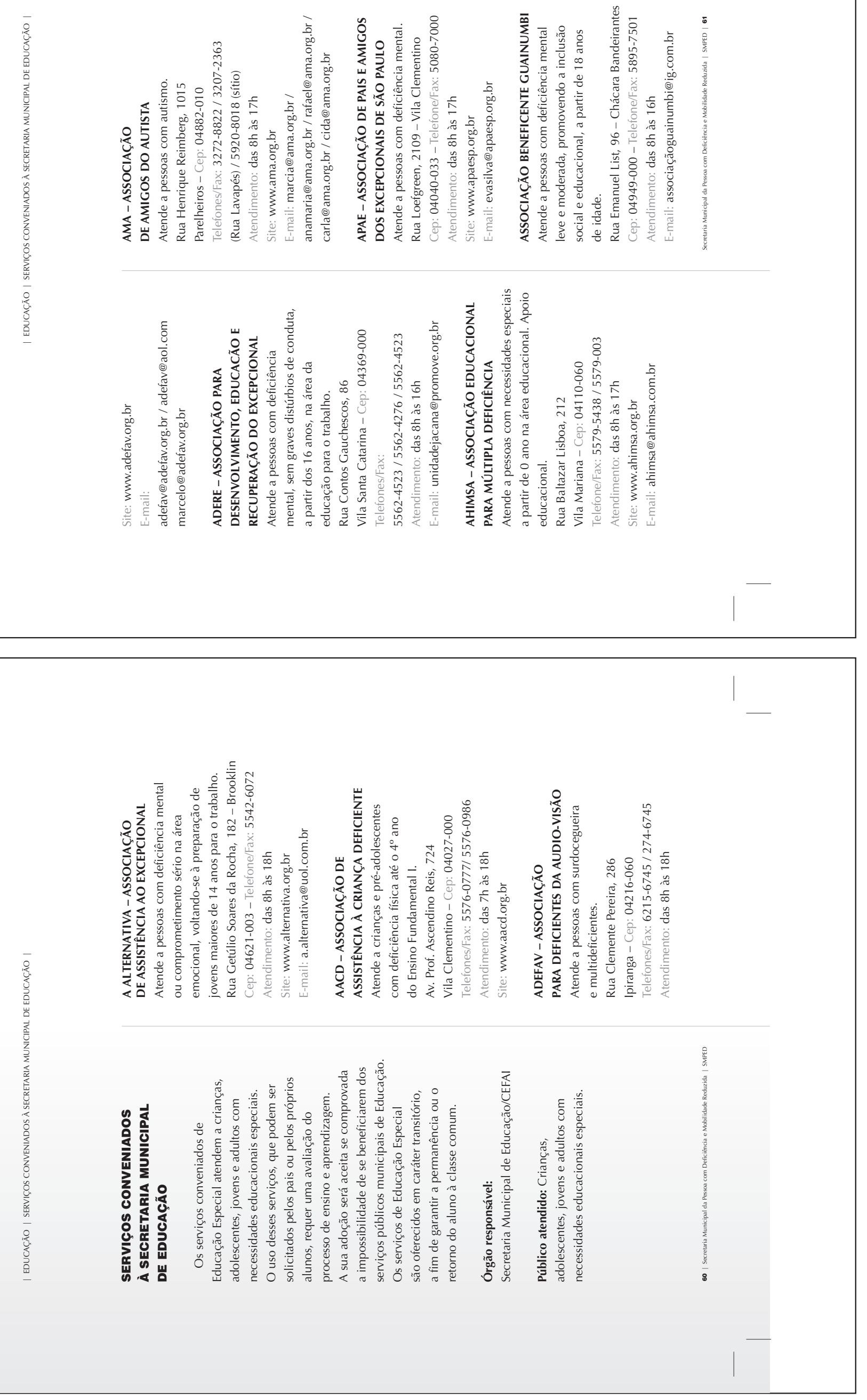

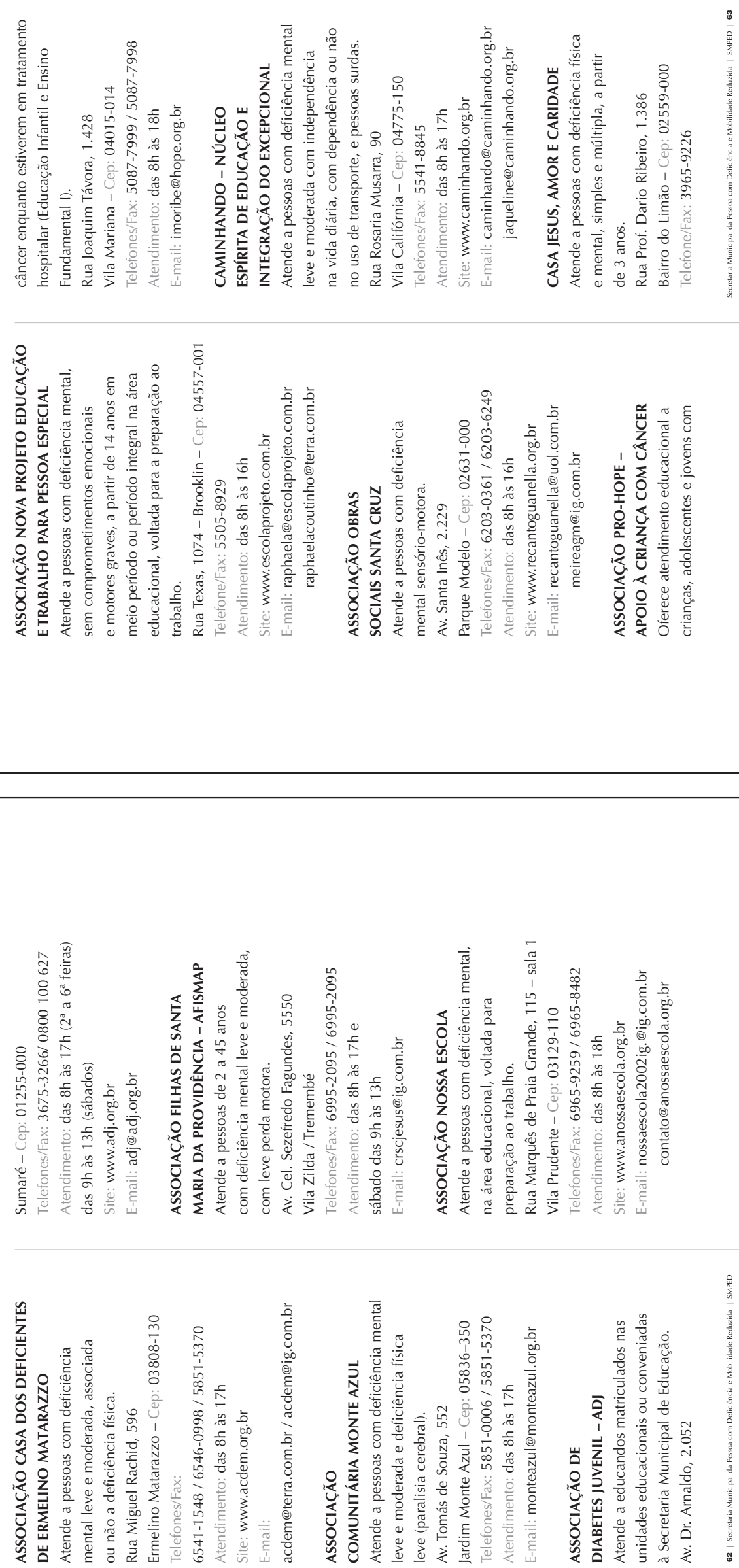

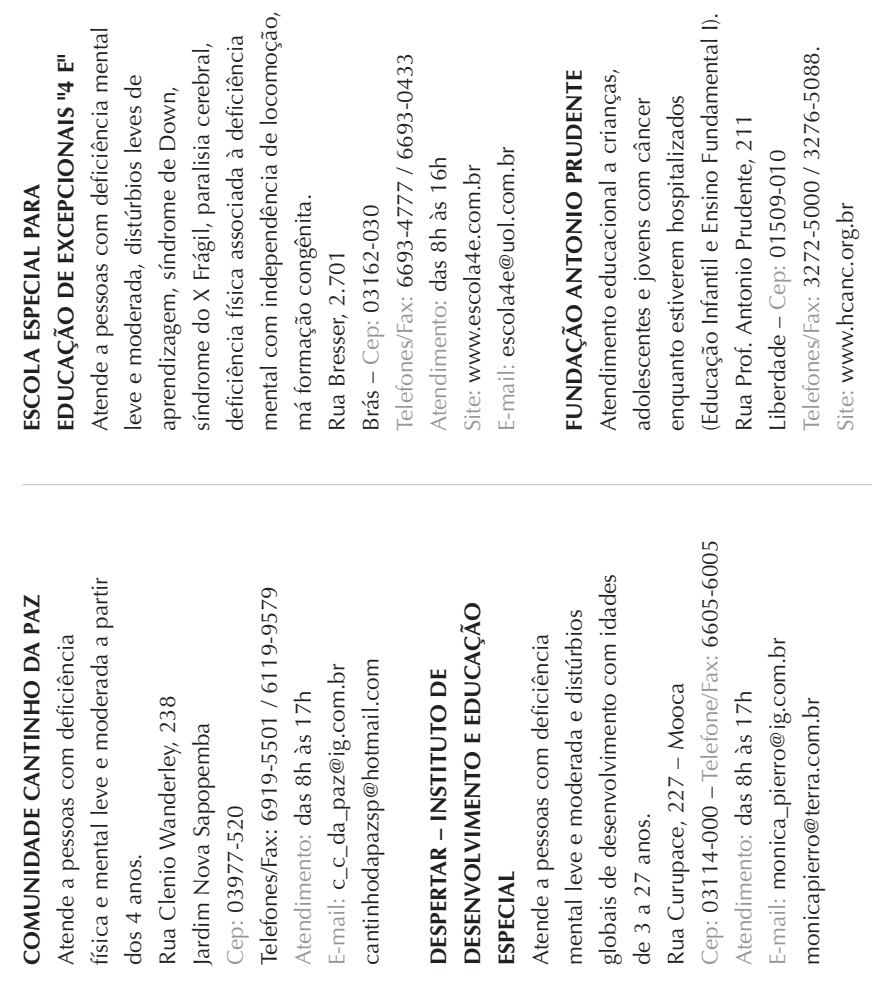

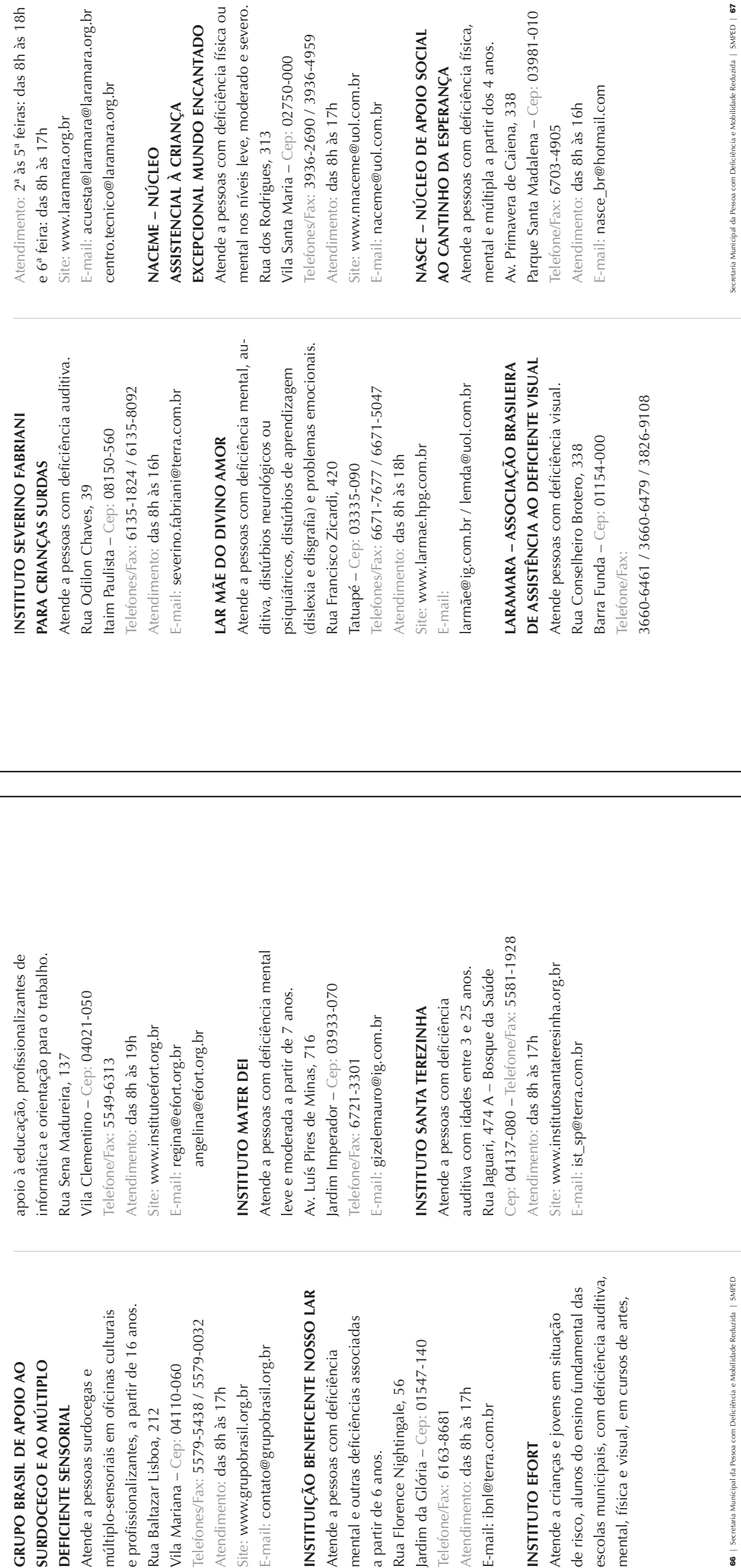

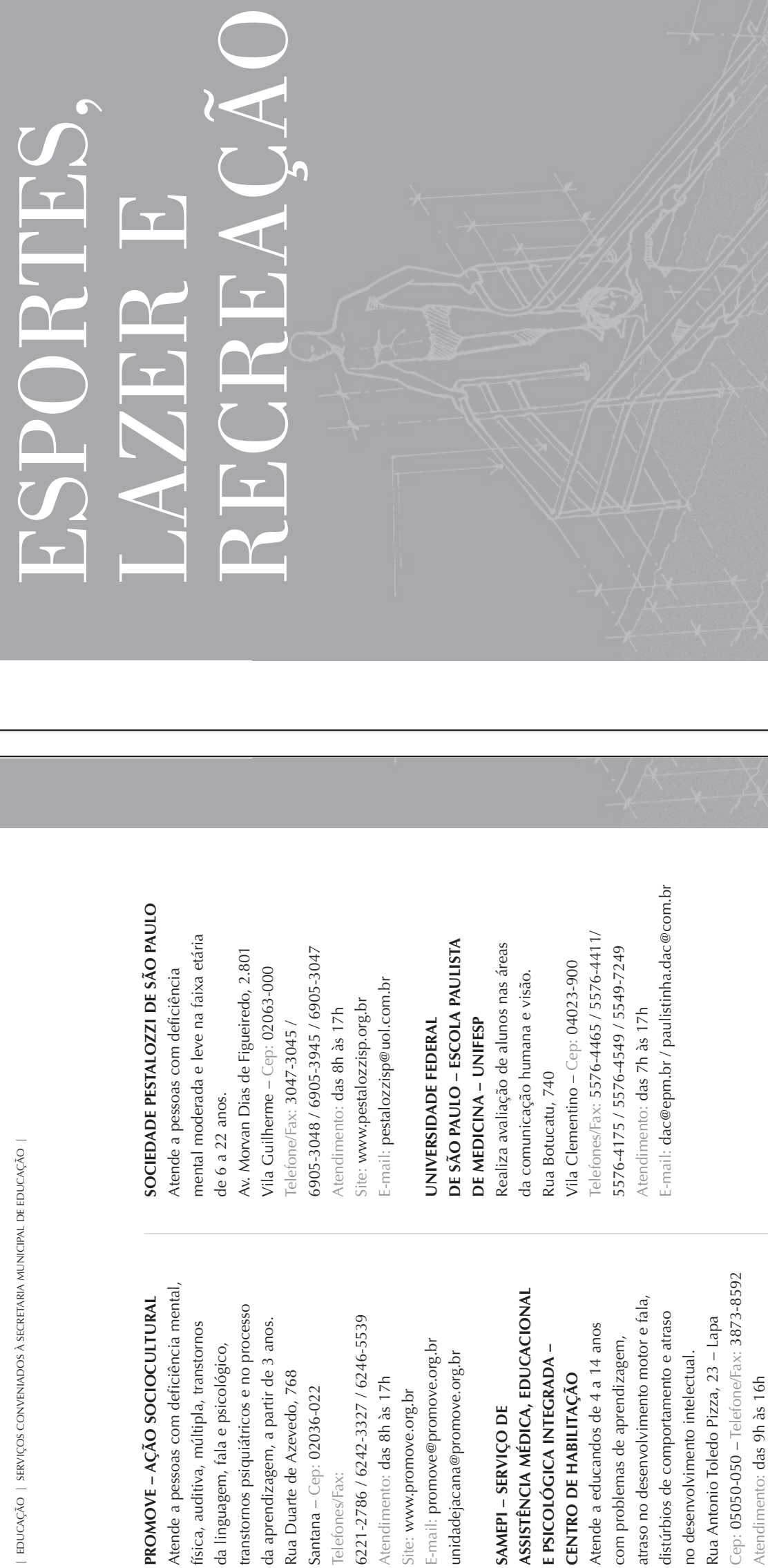

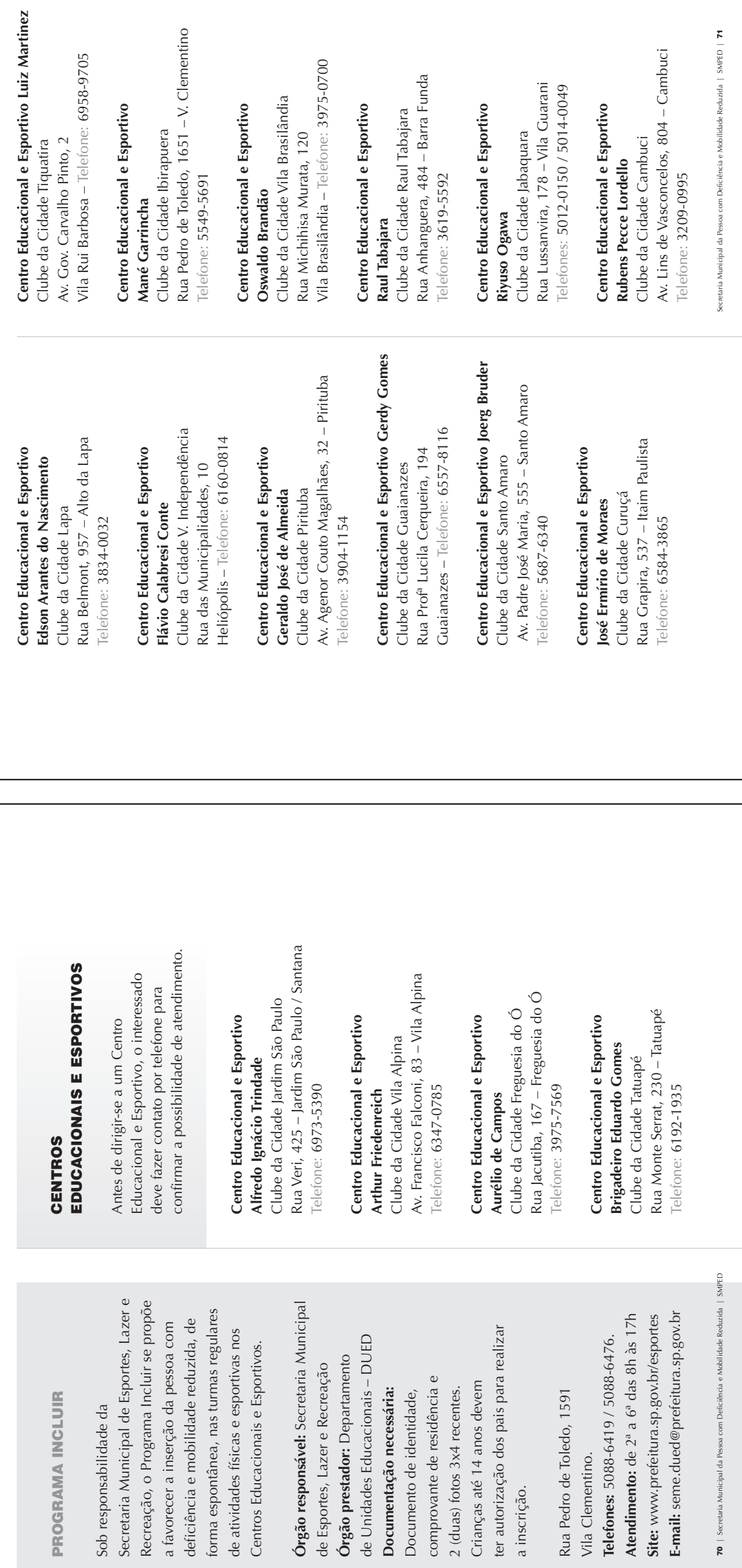

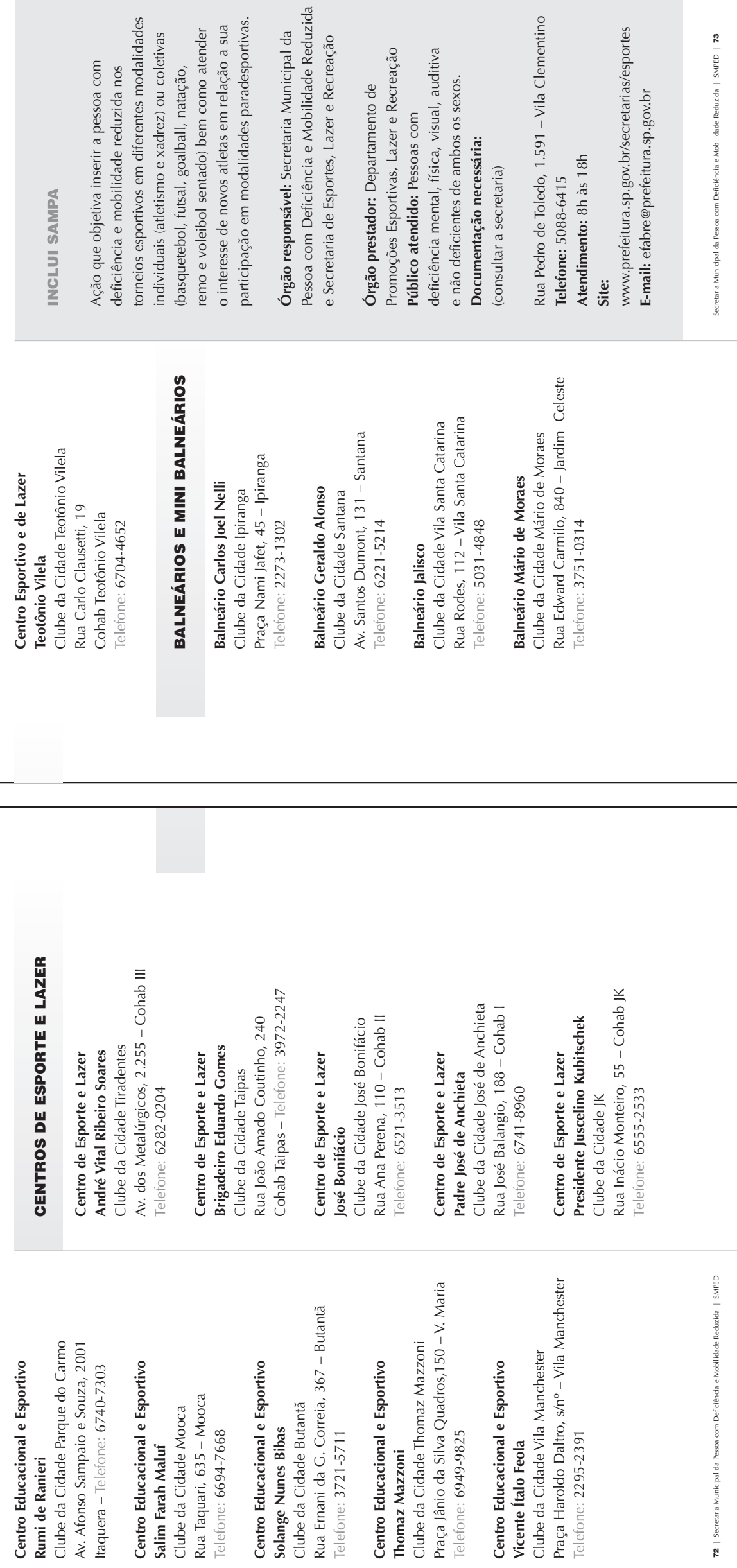

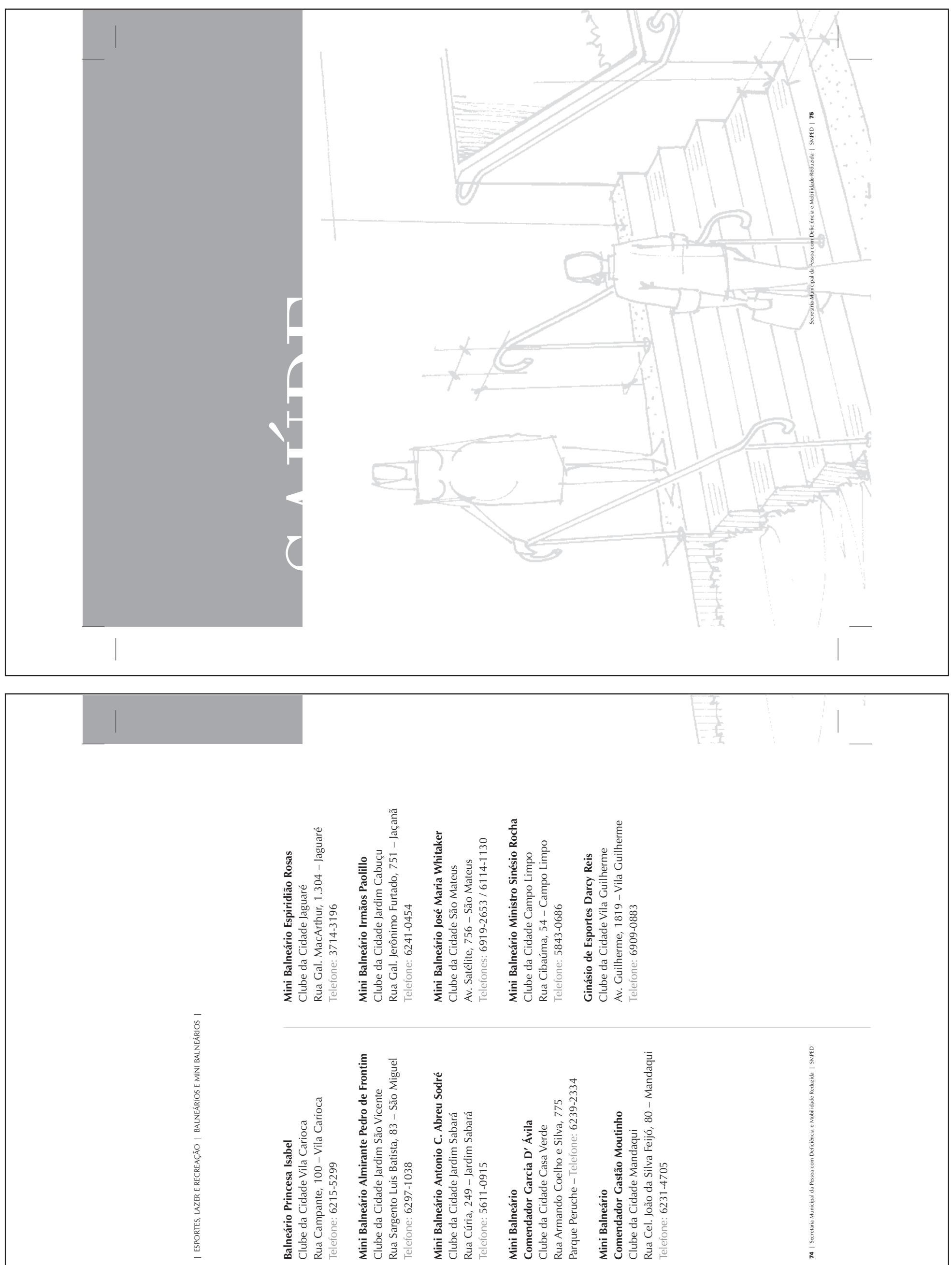

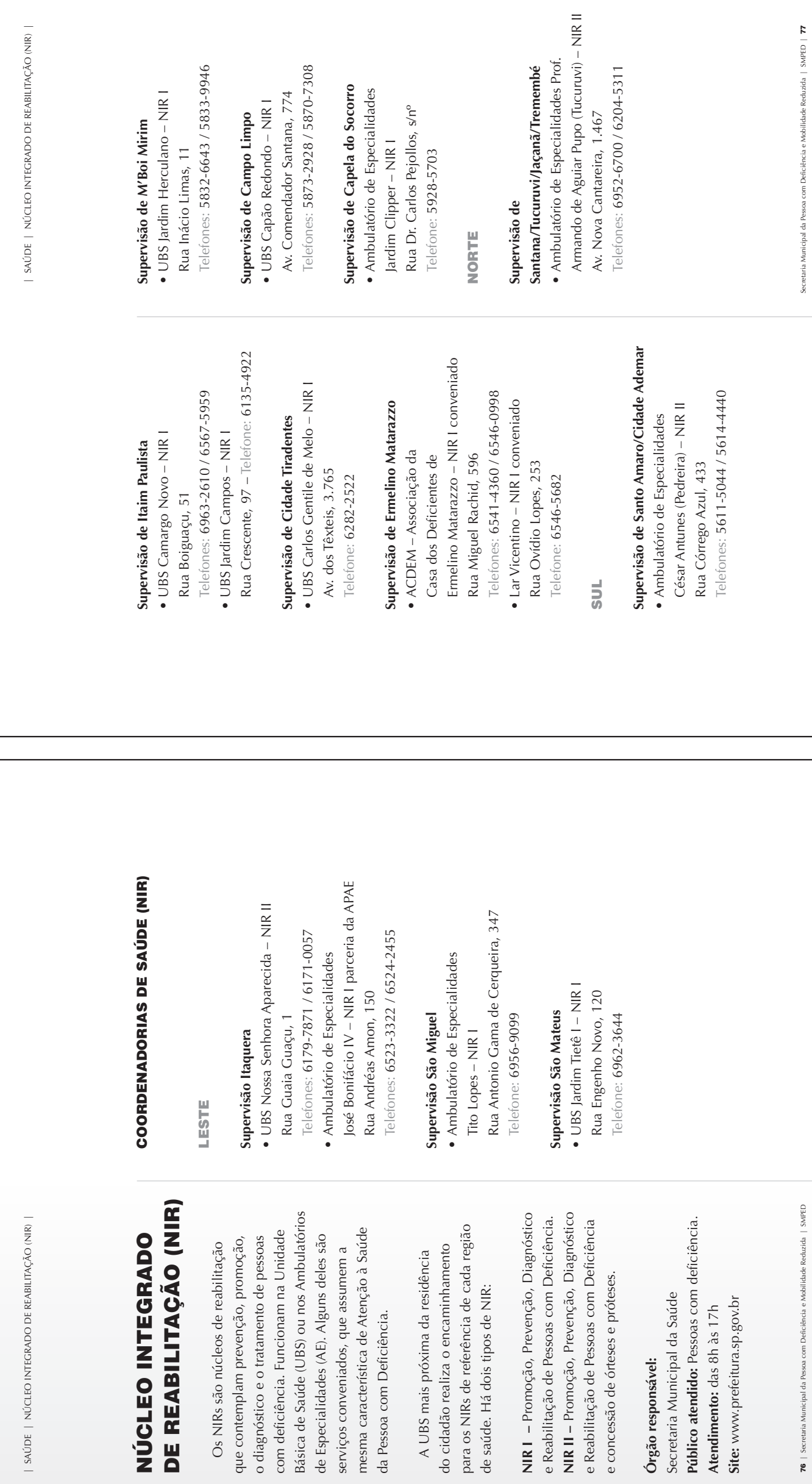

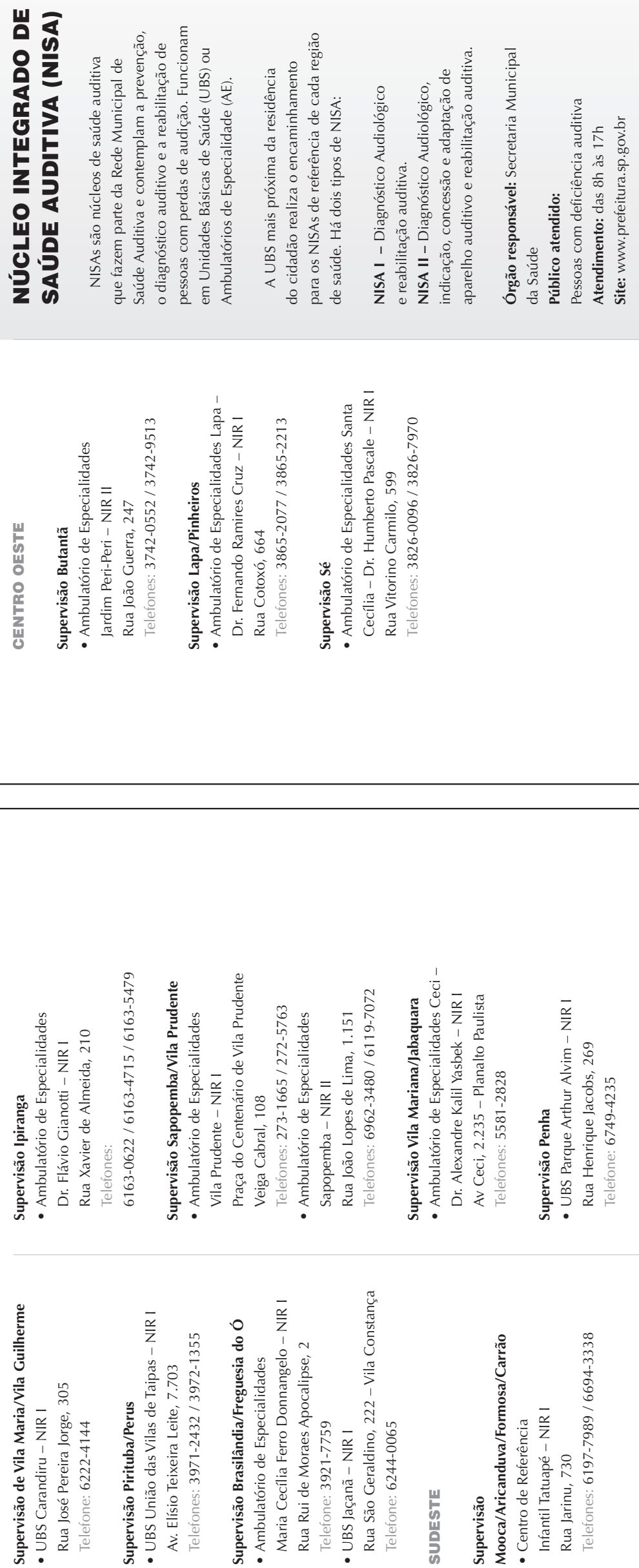

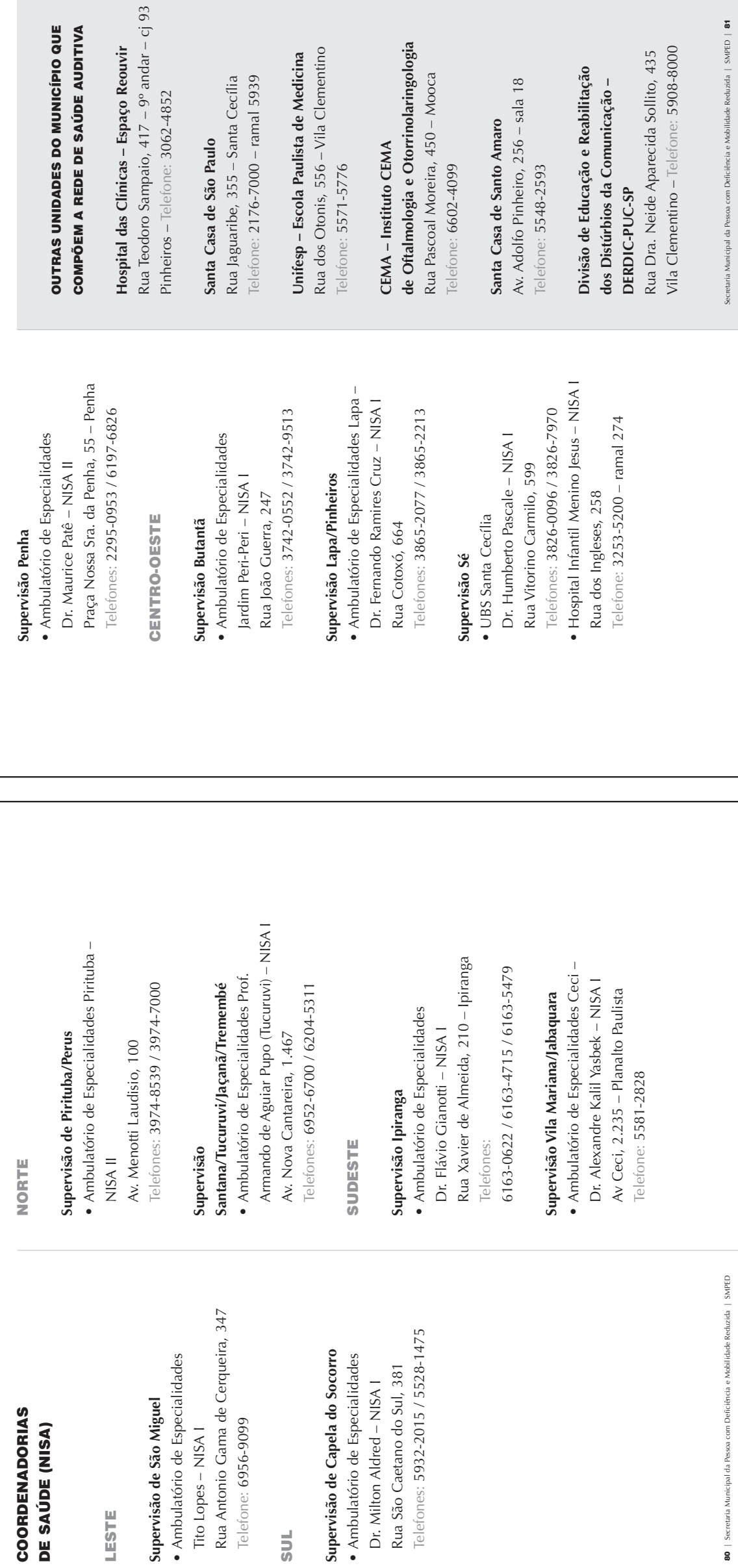

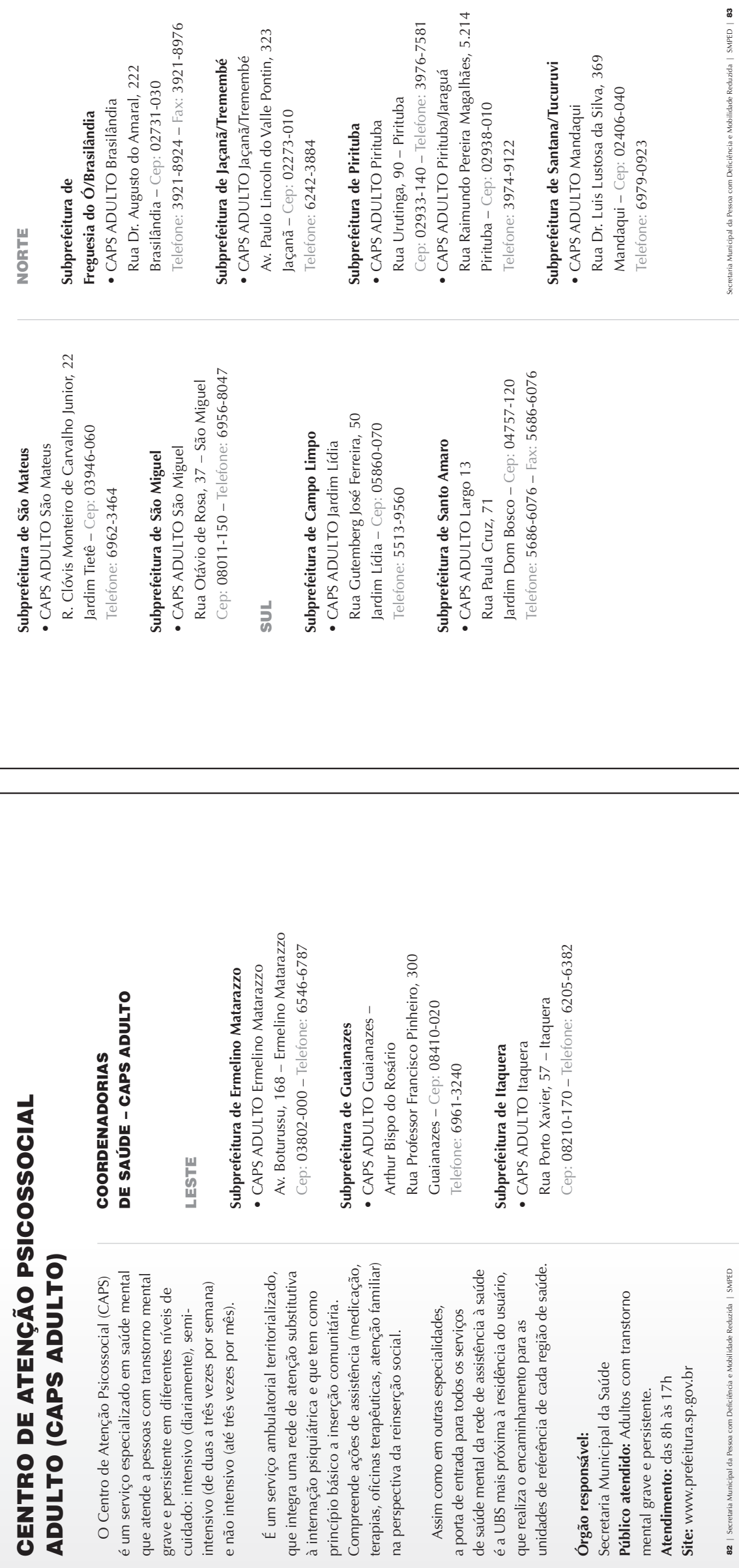

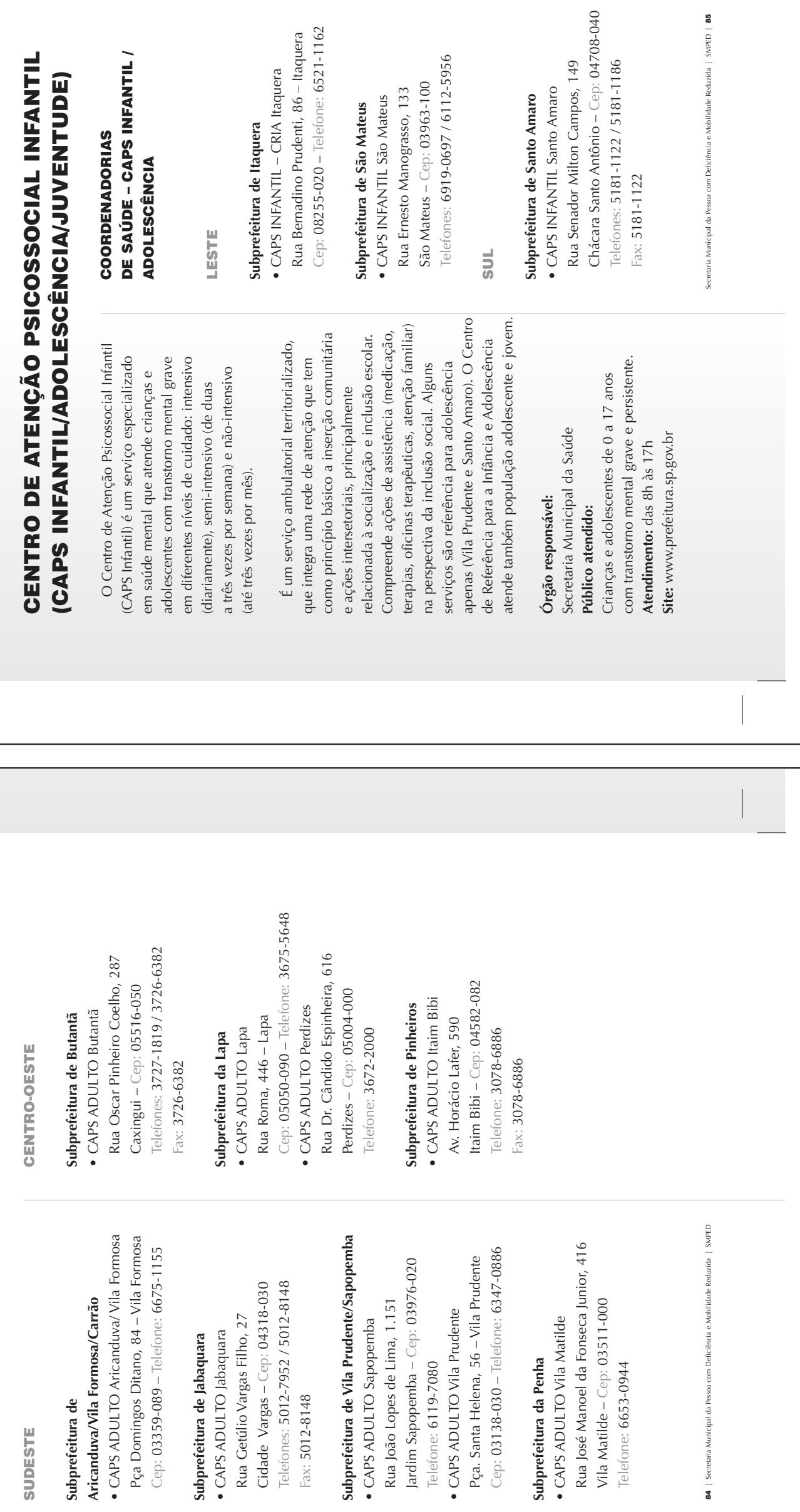

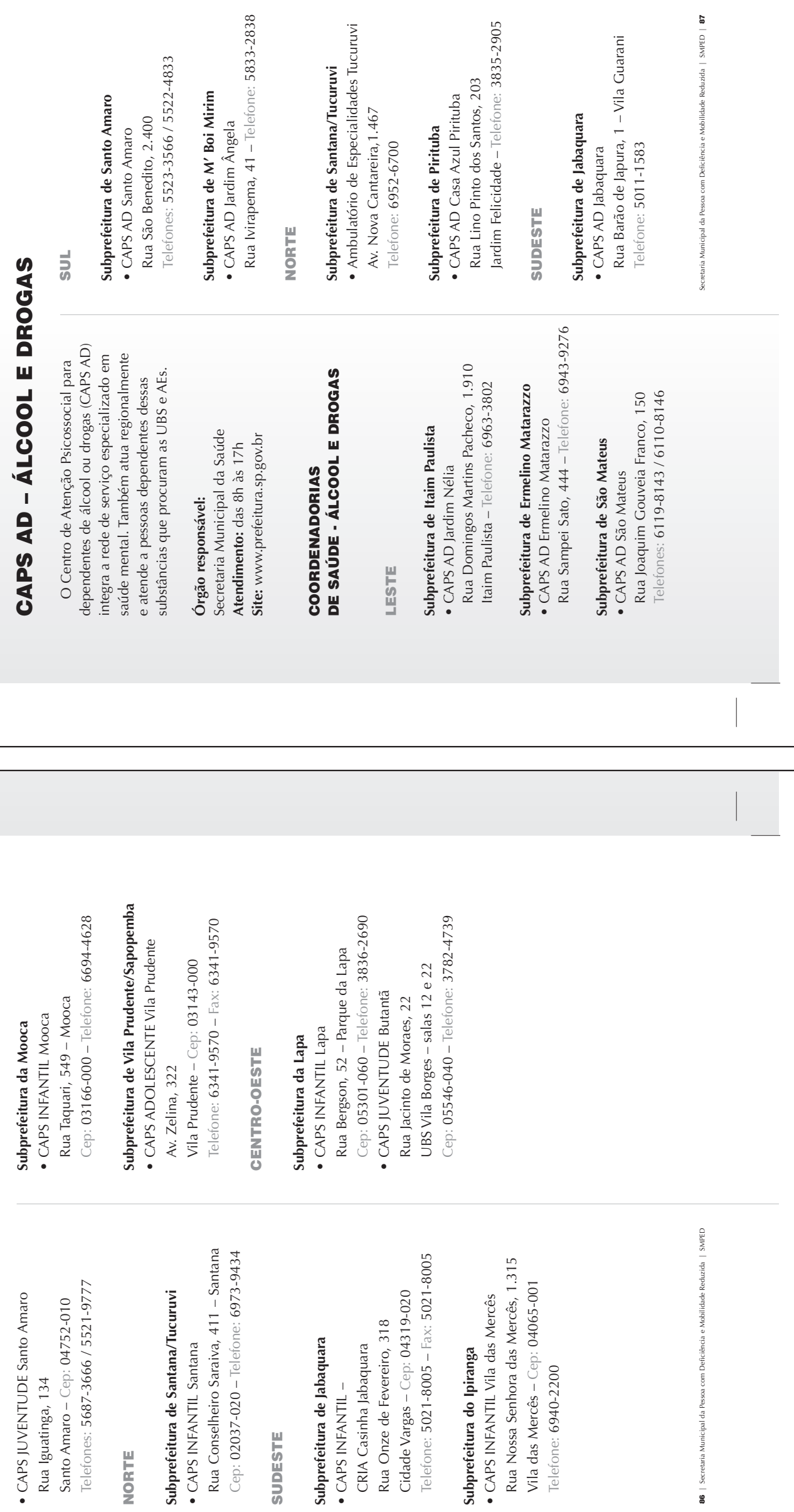

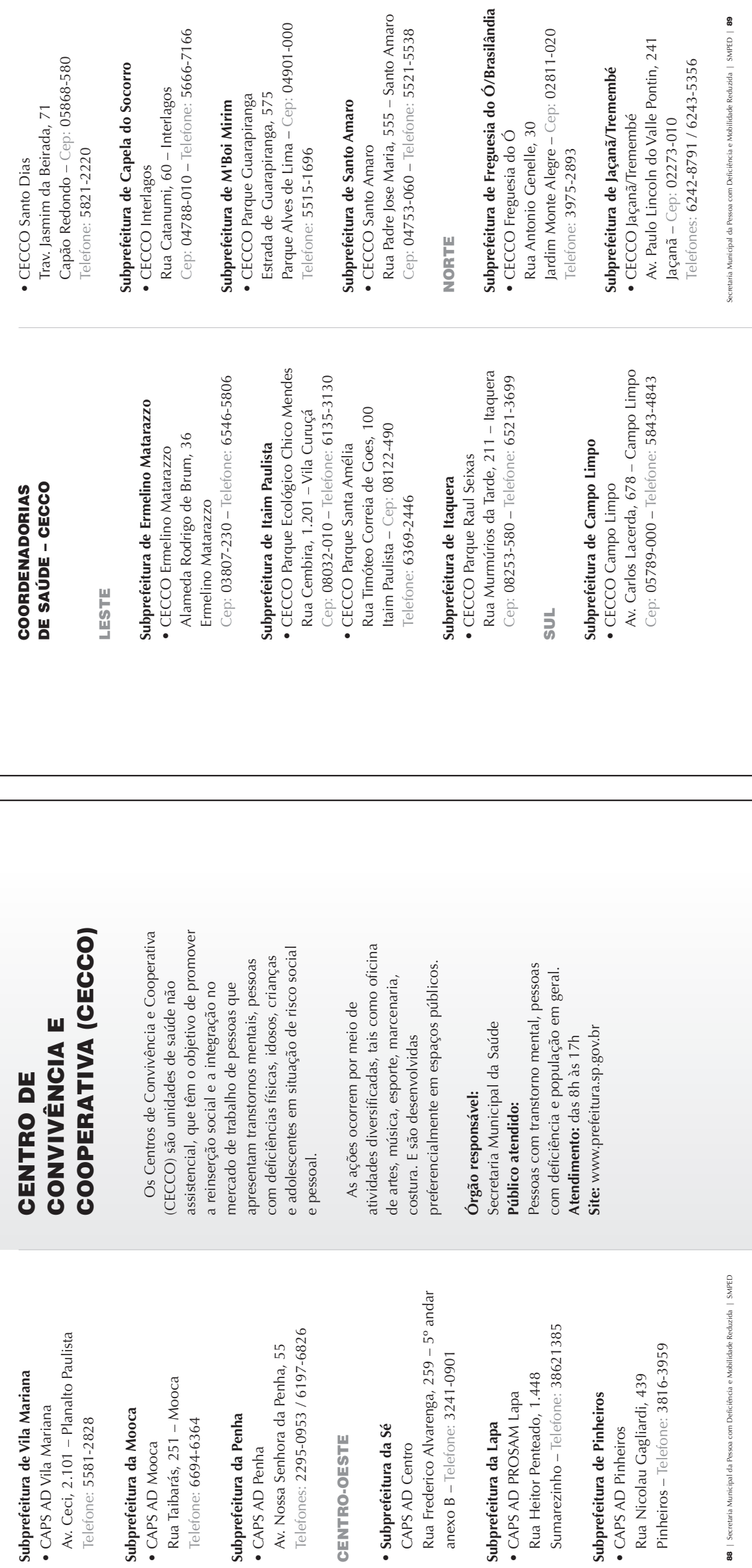

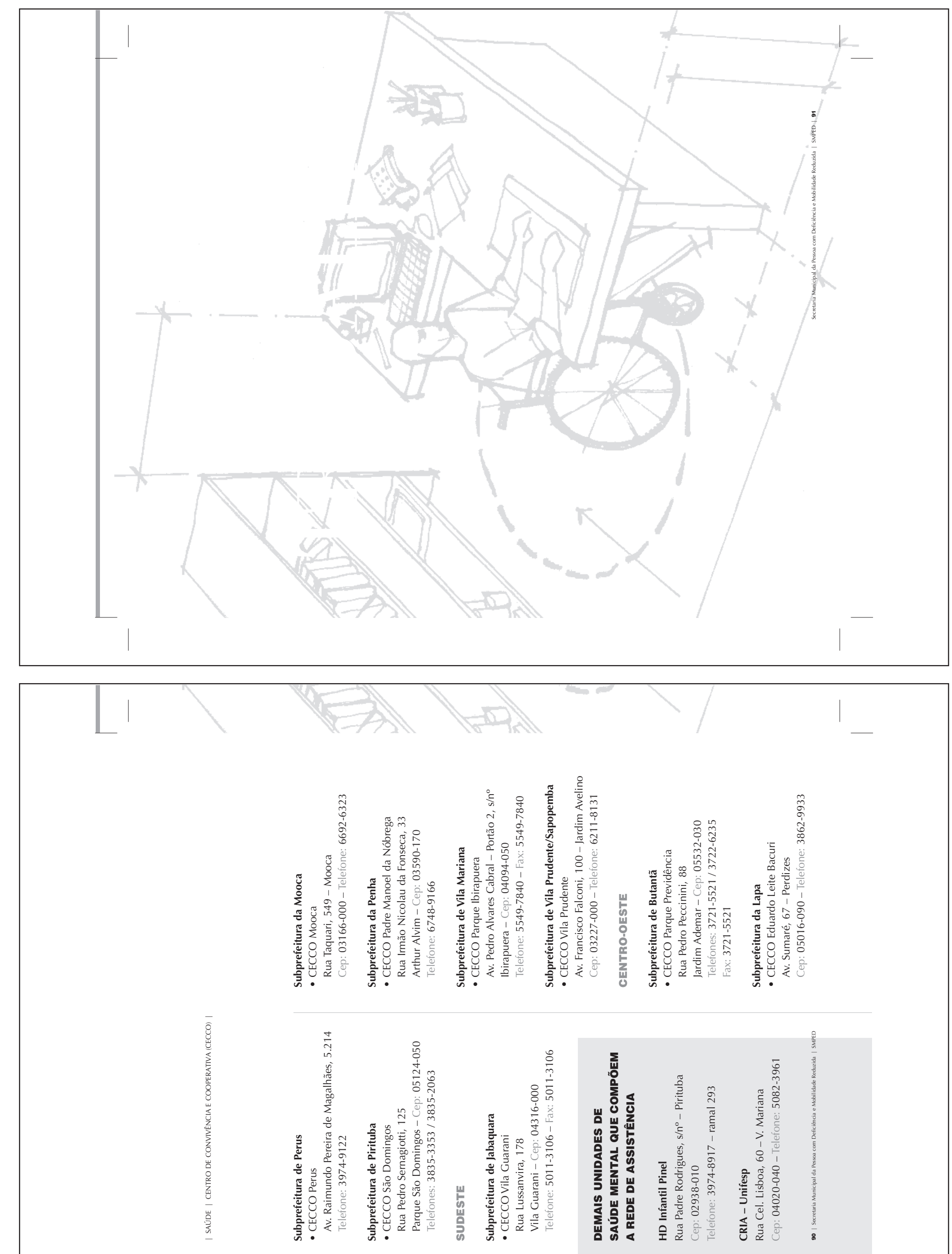

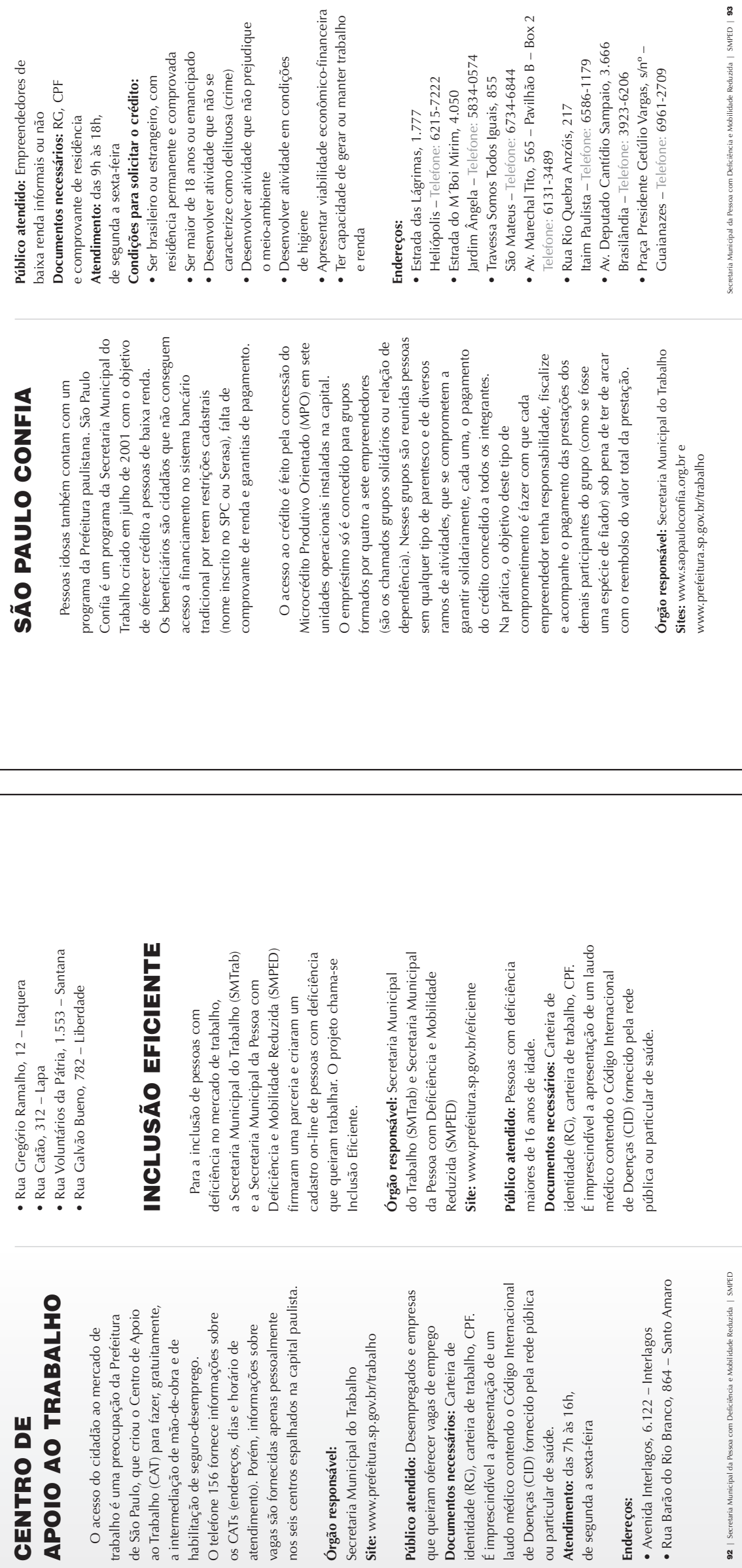

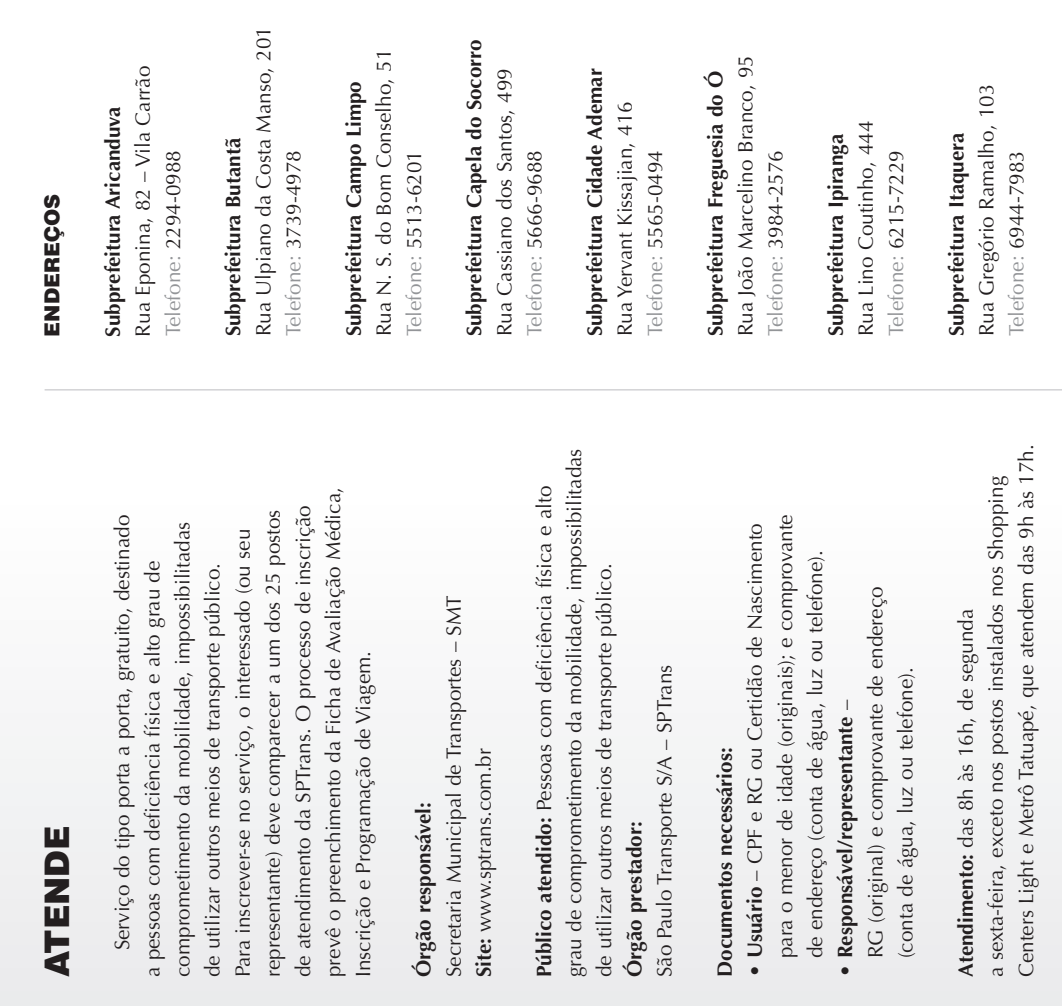

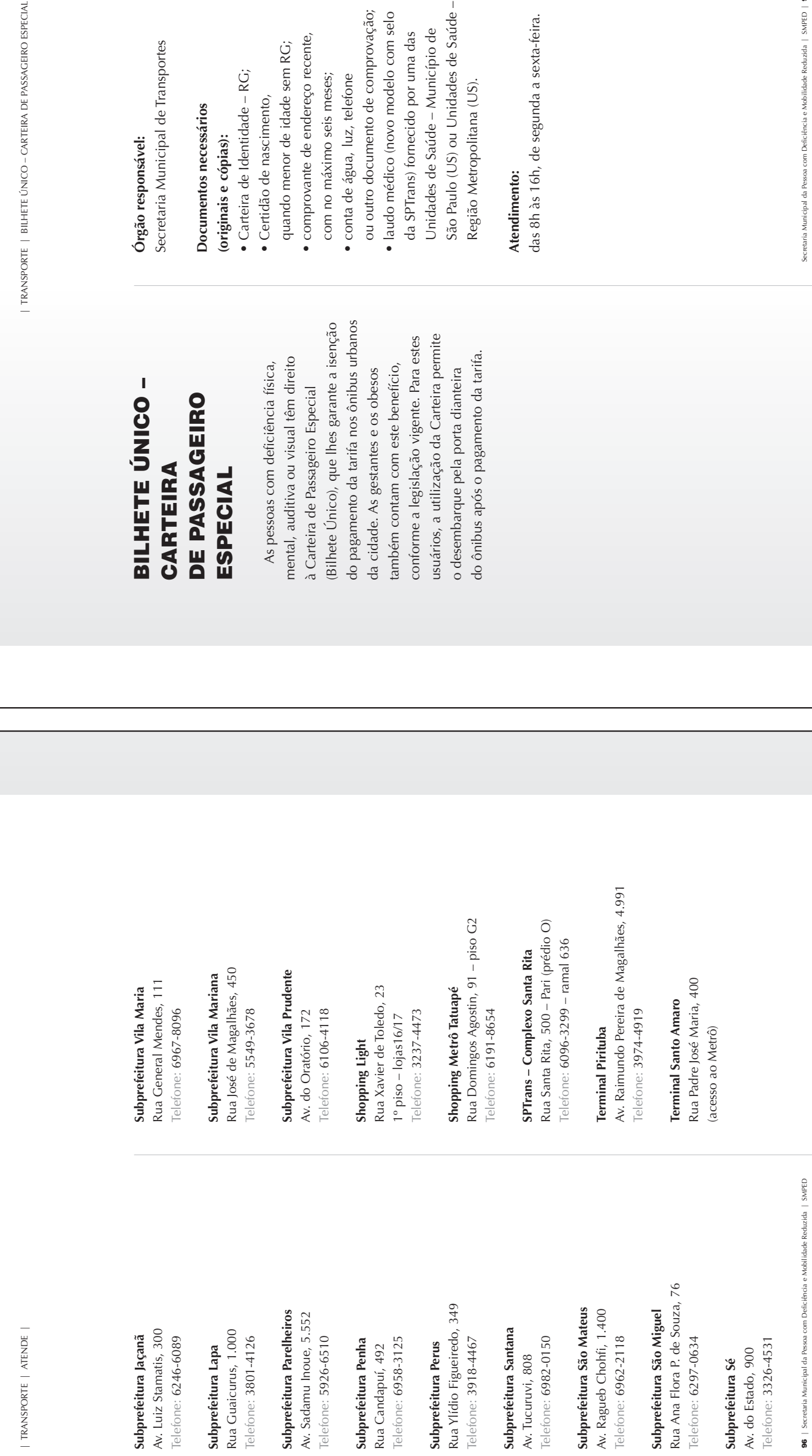

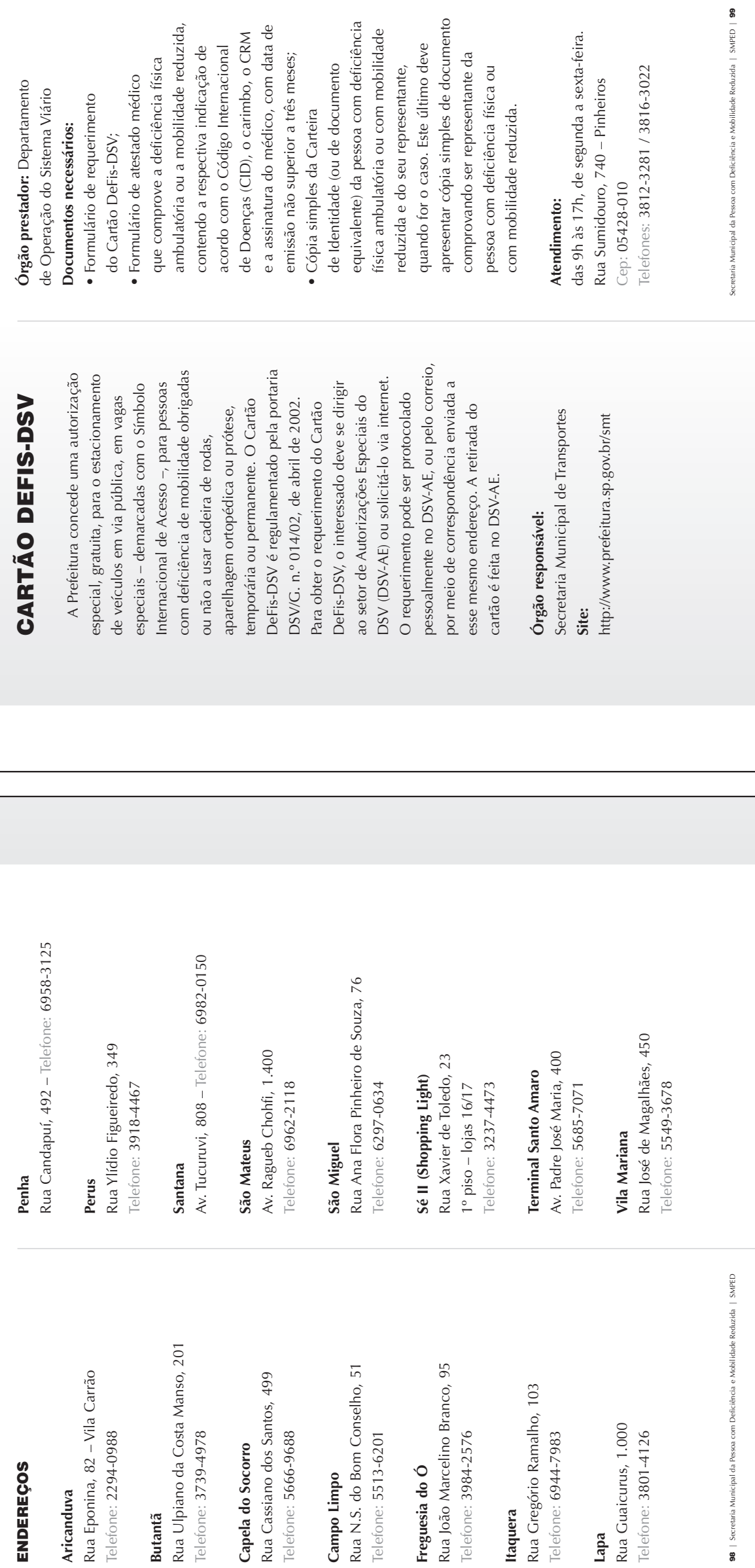

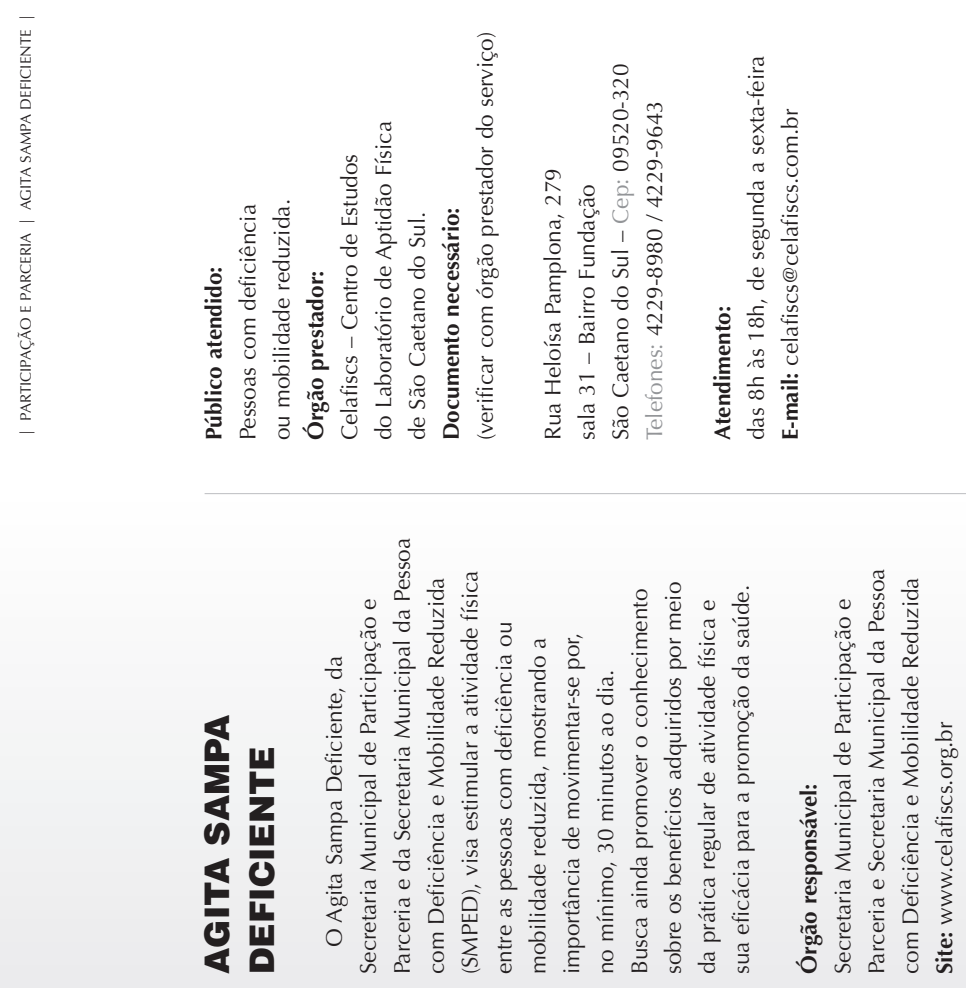

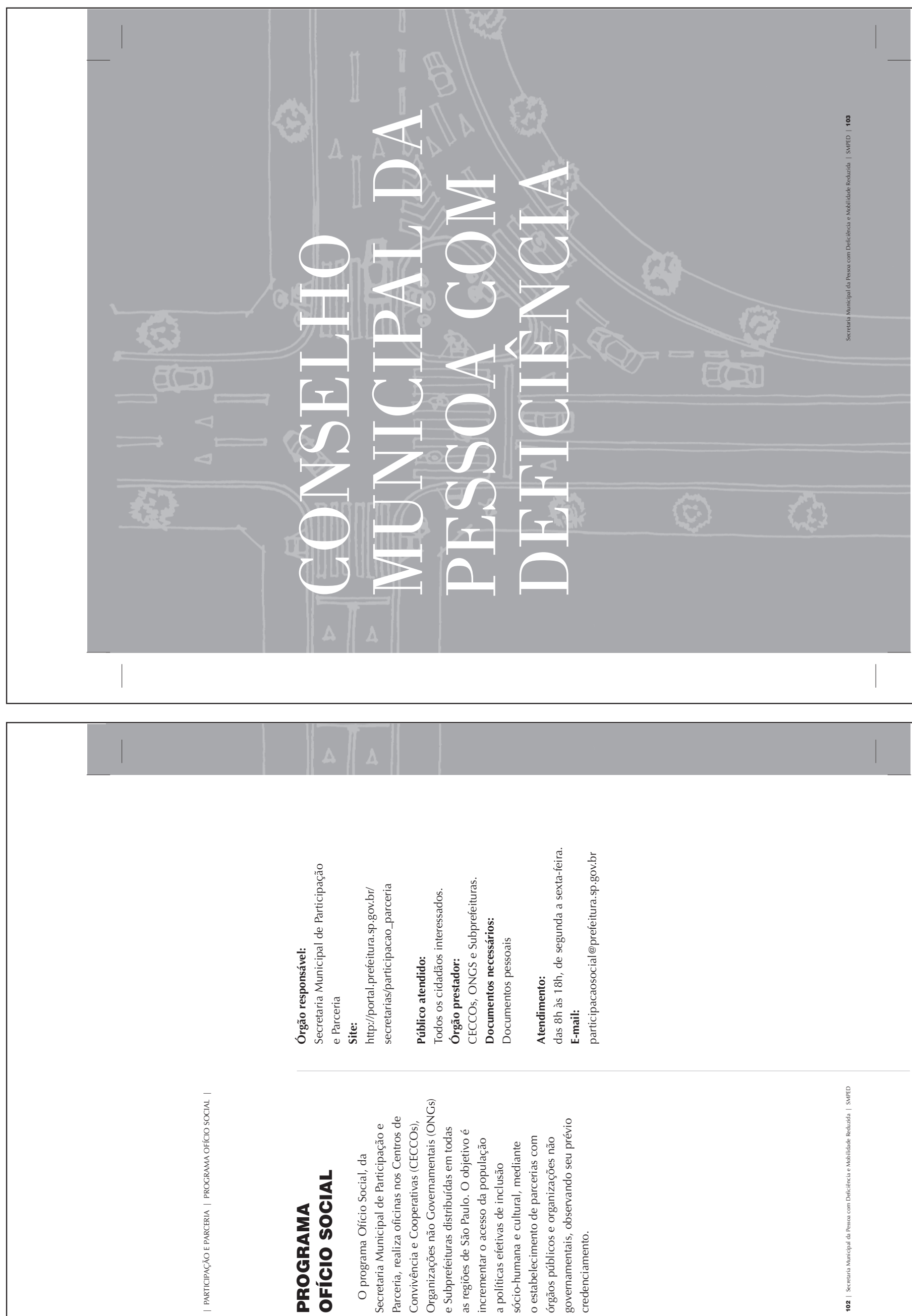

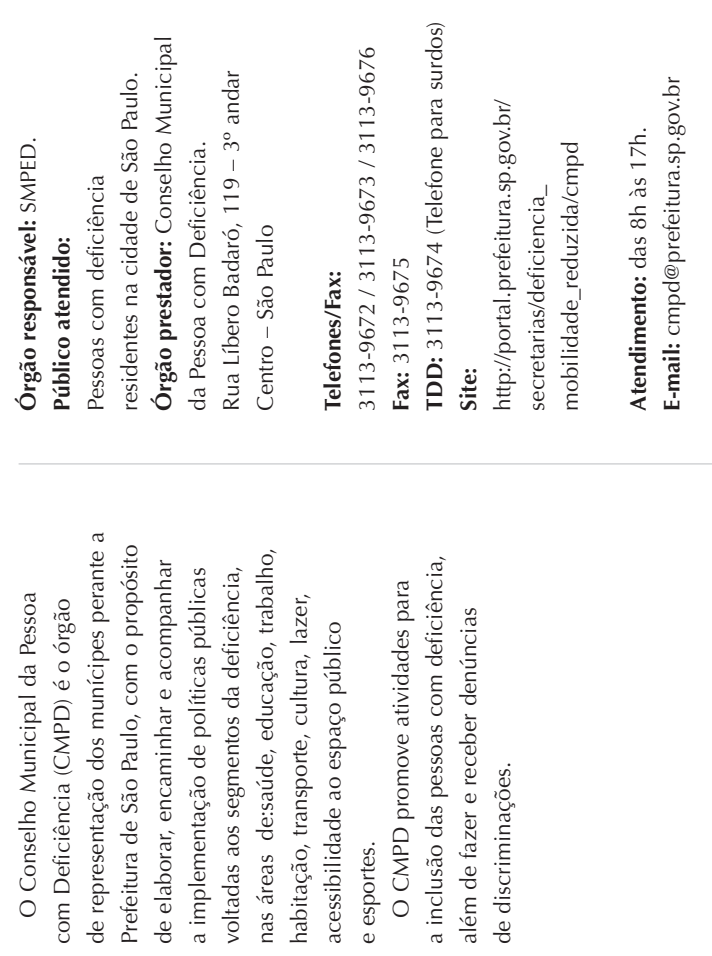